\title{
Water-soluble polyacetylene: a promising tool for sustainable drug delivery?
}

\author{
Saikat Mukherjee ${ }^{1}$ \& Raja Shunmugam*,1 \\ ${ }^{1}$ Polymer Research Centre, Department of Chemical Sciences, Indian Institute of Science Education \& Research Kolkata, \\ Mohanpur-741246, India \\ * Author for correspondence: sraja@iiserkol.ac.in
}

\begin{abstract}
"Prodrugs, a synthetic architecture, offer to employ different functionalities into its carrier part and deliver the small-molecule drug to the affected areas specifically by keeping the normal cells unaffected, which reduces the unwanted side effects of chemotherapy"
\end{abstract}

First draft submitted: 13 July 2017; Accepted for publication: 5 September 2017; Published online: 24 October 2017

Keywords: drug delivery • nanocarrier • nanomaterials • prodrug • site-specificity

\section{Cancer \& its therapies}

Cancer, which is characterized by abnormal cell growth, causes millions of deaths each year throughout the world [1]. However, the detailed mechanism of it is unknown and this partial understanding does not help toward finding a cure [1]. Many efforts have been made to treat cancer in different ways (surgery, immunotherapy, chemotherapy and radiation). Among these, chemotherapy is considered as the systematic treatment [2]. Chemotherapy deals with different single or multicomponent organic or inorganic molecules that are able to kill the cancer cells at nanomolar concentrations by following various different cellular mechanisms [3]. Organic anthracycline classes of compound (e.g., doxorubicin), alkaloid classes of compound (e.g., camptothecin), DNA alkylating agents (e.g., chlorambucil and paclitaxel) are considered as front-line chemotherapeutic agents for treating various cancers (e.g., head, neck, lung etc.). Platinum-based antineoplastic drugs also hold a unique position in the field of chemotherapy due to their efficient killing capabilities of tumor cells at very low concentrations [3]. Generally, these small molecules undergo different binding at the cellular level to cause apoptosis (programmed cell death), but the aqueous solubility and the inability of these small molecules to reach the tumor site causes enormous side effects that reduce the patient's survival rate [2]. Therefore, different models of prodrug systems have been explored to overcome the limitations generally faced by these small molecule chemotherapeutic agents [3].

\section{Prodrugs \& their utility}

Prodrugs, a synthetic architecture, offer to employ different functionalities into its carrier part and deliver the small-molecule drug to the affected areas specifically by keeping the normal cells unaffected, which reduces the unwanted side effects of chemotherapy [3-7]. Due to the abnormal growth of cancer cells, the cellular environment is different from the normal cellular environment in terms of $\mathrm{pH}$, temperature and enzymatic distribution [3]. So, the drug is being released from the carrier molecule by employing different stimuli responsive functionalities into the prodrug system, which eventually helps achieve a better therapeutic response over small molecules once it reaches the affected site.

Mainly two major methods have been commonly employed to deliver the drug, namely, covalent and noncovalent approaches [5-10]. Drug attached through different stimuli responsive covalent linkers (e.g., pH, thermal, photo) to the carrier molecule gives a therapeutic response over a longer period of time as it follows a sustained release mechanism, whereas noncovalent encapsulation of the drug follows the burst release mechanism [5-10]. There are a number of different prodrug systems that have been explored (e.g., liposome, polymer, peptide and nanoparticle), among these, polymer-based prodrug systems have recently been very popular. These systems show enhanced cytotoxic effects due to their plasma stability, which eventually helps it to increase its permeation and retention 
effect (EPR), and also due to the leaky vesicular nature of the blood vessels around the tumor site, which helps this nano-aggregate reach the targeted sites [4-12]. A polymer-based amphiphilic carrier molecule forms excellent nano-aggregates with different size and shapes in an aqueous environment (where it protects the hydrophobic drug moiety into its core), whereas the hydrophilic part (mostly polyethylene glycol [PEG]) of it forms the shell. The hydrophilic part of the carrier molecule can be further functionalized with different vitamins or minerals, which eventually helps the whole nanocarrier attach to the cancer cell surface with its overexpressed receptors [4-12].

\section{Site-specificity to reduce side effects}

It has been well explored that abnormally growing cancer cells take up huge amounts of vitamins, minerals and nutrients compared with normal cells, which further causes the formation of overexpressed receptors on the cancer cell surface. Folic acid and biotin (vitamin $\mathrm{H}$ ) are those molecules whose functionalization to the hydrophilic part of an amphiphilic polymer (mostly PEG) forms nano-aggregates with the pendent folate or biotin group in an aqueous environment. This further helps the whole nano-aggregate to be internalized through the receptor-mediated internalization pathway, which is a common pathway followed by the mammalian cells to engulf nanomaterials [4-12].

\section{Prodrugs \& their amphiphilicity}

This amphiphilic polymeric architecture also forms excellent nano-assemblies in aqueous environments with different shapes (e.g., spherical, vesicular and rod) at nanometer level. The shapes of these polymeric nanoaggregates in an aqueous environment are generally governed by the hydrophobic and hydrophilic balance [13]. By tuning the ratio of it, it is possible to tune the different shapes of the aggregates at a nanometer level, which actually influences the internalization kinetics. It has been well established that a rod like nano-aggregate can internalize better than a vesicular or spherical aggregate. Basically, the contact area between the aggregate and cellular surface plays a crucial role. So, a polymeric prodrug system can offer an improved alternative in terms of the site-specific delivery of chemotherapeutic agents [13].

\section{Different prodrug systems \& polyacetylene}

To enhance the pronounced effect of a chemotherapeutic agent, there is always a pressing need to have different delivery vehicles that will actually give the opportunity to anchor different functionalities to it in order to overcome the difficulties generally faced by the small drug molecules in real applications, for example, solubility, site-specificity etc. Different polymeric nanocarriers have already been explored by various research groups (e.g., norbornene, polycaprolactone, lactide, PEG and so on) but the polyacetylene based nanocarrier is not yet explored in the field of biomedicine, due to its nonliving nature, processability and intractability [4-10].

Polyacetylene was first produced by Natta [14], but the intractable white powder did not find any use due to its nonprocessability [14]. The different functional acetylenic moieties were also not very much explored in the same period of time. In the following decades, there were several efforts made to synthesize polyacetylene using different pathways: such as metathesis, cyclization, homocoupling, click polymerization etc. Among them, introduction of an organometallic catalyst gives a way to make polyacetylene in a living fashion with uniform distribution [15]. The formation of polyacetylene using an organometallic molybdenum catalyst was first reported by Fox and Schrock [16]. This opened up a new avenue in the field of cyclopolymerization of polyacetylene [16]. The catalyst was efficient enough to make polyacetylene from 1,6-hepta di-yne in a living fashion with uniform distribution. 1,6-hepta di-yne is an important monomer for functionalizing different useful moieties for the purpose of site-specific delivery of chemotherapeutic agents [16]. It was further reported that this particular monomer can even be polymerized to form polyacetylene with the use of a functional group tolerant Ruthenium catalyst, modified by Choi et al. with Ru-based catalyst mainly Grubbs' catalyst to give superior control over polymerization [17-19]. Further, due to the presence of an extended conjugated backbone, this polyacetylene shows excellent conducting and optoelectronic properties. This backbone also shows excellent morphology due to self-assembly at the nanoscale [20]. The synthesis of polyacetylene in large-scale with processable functionality actually suffers from reactivity of the carbine intermediate formed in propagating steps. Choi et al. showed that the control over molecular weight and poly dispersity index (PDI) can be achieved using highly reactive Grubbs' third-generation catalyst. Similarly, they have also demonstrated that first-generation Grubbs' catalyst, with the addition of additives in small amounts, can do the same [17-19]. So, this highly conjugated biocompatible backbone can be used in the field of biomedicine as the backbone itself can be used as an imaging modality. 
Specifically, the potential molecule gem-1,6-hepta di-yne gives the opportunity to open up a new polymeric backbone for its capability of making polymers with controlled molecular weight and uniform distribution. The disubstituted ester group can be fine-tuned to anchor different functionalities for its solubility and serves the purpose as a nanocarrier for different drugs to improve their performance. The drug can be covalently attached with different stimuli responsive linkers at the monomeric level along with different functionality for solubility and site-specificity, and for better performance. With this kind of system, we can get a site-specific nanocarrier that follows sustained release once it reaches the tumor site. Also, the rigid backbone of 1,6-hepta di-yne offers excellent different morphology at the nanoscale level, which can be utilized for the noncovalent encapsulation of chemotherapeutic agents for better cellular internalization [20,21].

\section{Conclusion \& future perspective}

In this era, this drug-delivery system is being modified as a theranostic nanosystem, for a better understanding of the therapeutic pathway that will eventually help to improve therapeutic efficacy [22]. Therefore, by combining both its imaging modality and chemotherapeutic agent abilities into a single system, this polyacetylene backbone can serve its purpose as an imaging unit. Due to its extended conjugation, this molecule shows excellent color in the visible region and can be used as an imaging motif in order to create a site-specific nanotheranostic tool [5,22]. This new backbone opens up different possibilities for synthesizing complex architecture with improved control over the properties, for the purpose of improved therapy in modern medicine.

Financial \& competing interests disclosure

S Mukherjee thanks CSIR New Delhi, for their research fellowships. R Shunmugam thanks the Department of Science and Technology, New Delhi, for the Ramanujan Fellowship and DST-SERB (EMR/2015/001600) for funding. R Shunmugam thanks IISER-Kolkata for supplying infrastructure and start-up funding. The authors have no other relevant affiliations or financial involvement with any organization or entity with a financial interest in or financial conflict with the subject matter or materials discussed in the manuscript apart from those disclosed.

No writing assistance was utilized in the production of this manuscript.

\section{References}

1 Siegel RL, Miller KD, Jemal AD. Cancer statistics, 2016. Cancer J. Clin. 66, 7-30 (2016).

2 Miller KD, Siegel RL, Lin CC et al. Cancer treatment and survivorship statistics, 2016. Cancer J. Clin. 66, 271-289 (2016).

3 Chabner BA, Roberts TG Jr. Chemotherapy and the war on cancer. Nat. Rev. Cancer 5, 65-72 (2005).

4 Peer D, Karp JM, Hong S, Farokhzad OC, Margalit R, Langer R. Nanocarriers as an emerging platform for cancer therapy. Nat. Nanotechnol. 2, 751-760 (2007).

5 Mukherjee S, Dinda H, Chakraborty I, Bhattacharyya R, Das Sarma J, Shunmugam R. Engineering camptothecin-derived norbornene polymers for theranostic application ACS Omega 2(6), 2848-2857 (2017).

6 Bhattacharya S, Ganivada MN, Dinda H, Das Sarma J, Shunmugam R. Biodegradable copolymer for stimuli-responsive sustained release of doxorubicin. ACS Omega 1, 108-117 (2016).

7 Mukherjee S, Das Sarma J, Shunmugam R. pH-sensitive nanoaggregates for site-specific drug-delivery as well as cancer cell imaging. ACS Omega 1, 755-764 (2016).

8 Mane SR, Rao VN, Chaterjee K et al. Amphiphilic homopolymer vesicles as unique nano-carriers for cancer therapy. Macromolecules 45(19), 8037-8042 (2012)

9 Mukherjee S, Patra D, Dinda H et al. Super paramagnetic norbornene copolymer functionalized with biotin and doxorubicin: a potential unique site-specific theranostic agent. Macromolecules 49, 2411-2418 (2016).

10 Saha B, Haldar U, De P. Polymer-chlorambucil drug conjugates: a dynamic platform of anticancer drug delivery. Macromol. Rapid Commun. 37, 1015-1020 (2016).

11 Kapri S, Maiti S, Bhattacharyya S. Lemon grass derived porous carbon nanospheres functionalized for controlled and targeted drug delivery. Carbon 100, 223-235 (2016).

12 Homyak C, Anson F, Thayumanavan S. Supramolecular polymers in nanomedicine. Comprehensive Supramolecular Chemistry II, Elsevier Inc.Atwood JW (Ed.). (2016) doi:10.1016/B978-0-12-409547-2.12566-1

13 Gratton SE, Ropp PA, Pohlhaus PD et al. The effect of particle design on cellular internalization pathways. Proc. Natl Acad. Sci. USA 105(33), 11613-11618 (2008).

14 Natta G, Atthi Accad naz. Lincei, Rend Classe. Stereospecific polymerization of acetylene. Sci. Fis. Mat. Nat. 3, 25 (1958).

15 Aldissi M. Review of the synthesis of polyacetylene and its stabilization to ambient atmosphere. Synth. Met. 9, 131-141 (1984). 
16 Fox $\mathrm{HH}$, Schrock RR. Living cyclopolymerization of diethyl dipropargylmalonate by Mo(CH-t-Bu)(NAr) $[\mathrm{OCMe}(\mathrm{CF} 3) 2] 2$ in dimethoxyethane. Organometallics 11, 2763-2765 (1992).

17 Kang E-H, Kang C, Yang S, Oks E, Choi T-L. Mechanistic investigations on the competition between the cyclopolymerization and $(2+2+2)$ cycloaddition of 1,6-heptadiyne derivatives using second-generation Grubbs' catalysts. Macromolecules 49(17), 6240-6250 (2016).

18 Kang E-H, Yu SY, Lee IS, Park SE, Choi T-L. Strategies to enhance cyclopolymerization using third-generation Grubbs' catalyst. J. Am. Chem. Soc. 136, 10508-10514 (2014).

19 Kang C, Kang E-H, Choi T-L. Successful cyclopolymerization of 1,6-heptadiynes using first-generation Grubbs' catalyst twenty years after its invention: revealing a comprehensive picture of cyclopolymerization using Grubbs' catalysts. Macromolecules 50, 3153-3163 (2017).

20 Yang S, Shin S, Choi I, Lee J, Choi T-L. Direct formation of large-area 2D nanosheets from fluorescent semiconducting homopolymer with orthorhombic crystalline orientation. J. Am. Chem. Soc. 139, 3082-3088 (2017).

21 Ganivada MN, Kumar P, Babu A, Das Sarma J, Shunmugam R. Engineering a new class of multiarm homopolymer for sustainable drug delivery. ACS Biomater. Sci. Eng. 3, 903-908 (2017).

22 Mukherjee S, Shashank L, Dinda H et al. Site-specific amphiphilic magnetic copolymer nano-aggregates for dual imaging. Macromolecules 48(19), 6791-6800 (2015). 


\title{
A complete world of design adaptation and product specificities for injectable drug delivery
}

\author{
Pascale Gauthier*,1 \\ ${ }^{1}$ Biopharmaceutics \& Pharmaceutical Technology Department, Auvergne University, UMR 4045 Medis, 28, Place H. Dunant, B.P. \\ 38, F-63001 Clermont-Ferrand, France \\ * Author for correspondence: pascale.gauthier@udamail.fr
}

"The digital era has become a reality, fully integrated in all fields as it provides a better monitoring of treatment and the best explanation on chronic disease. A simple add-on solution to record and transmit injection data allows the creation of a long-term health benefit that can decrease the costs of healthcare and introduce a new interface with patients."

First draft submitted: 15 June 2017; Accepted for publication: 5 September 2017; Published online: 24 October 2017

Keywords: compliance injectable forms • design adaptation • packaging • safety in use

The world of injectable products is in good health with one-digit growth in Europe and a strong two-digit growth at international level (the USA and a few emerging countries). The global market of injection devices is expected to increase from $\$ 11.6$ bn in 2013 to $\$ 17.5$ bn in 2018 , and self-injection devices have shown the highest growth rate of $16.1 \%$ compared with conventional injectable forms [1]. Two elements can explain why the injectable market has seen such considerable growth: the rising prevalence of chronic diseases that can be treated with injection; and new treatments with biosimilars and biotechnologies presented in injectable forms. After rules and definitions, a short overview of some drug-design adaptations proposed for injectable forms is presented and emphasizes how this sector is innovative for offering clever systems that can adapt to both the user and product.

\section{Injectable definitions, particularities \& rules}

Historically, the term parenteral refers to injectable route and derives from the Greek para (outside) and enteron (intestine). Parenteral administration involves the injection of therapeutic agents, in the form of solution, suspensions or emulsions that must be sterile and pyrogen-free. Parenteral forms were officially recognized in the mid-19th century, when the morphine solution appeared in 1874 (Addendum in British Pharmacopeia [BP] 1867) [2]. As a key point definition (given by European Pharmacopeia or USP) [3,4], parenteral forms are sterile preparations intended for administration by injection, infusion or implantation, that may require the use of excipients with specific requirements (asepticity, nonpyrogenicity and $\mathrm{pH}$ ). The injectable route still remains the most rapid and direct delivery route, as it requires the right product in the right amount (any correction after the injection is impossible) with active delivery into the body occurring after skin penetration. Injection avoids the problems of deglutition or degradation of active drug in the gastrointestinal tract. Through this delivery method, drug administration to an unwilling (or unconscious) patient is also possible, which can be helpful in an emergency use. One limitation of this method is the risk of a needle stick injury, which still remains a problem as pain must be limited and any misuse or confusion in the drug or dosage administered must be avoided. Due to the risk of introducing contaminants into the body, injection represents the highest risk of infection out of all the drug delivery methods currently available. For all these reasons, parenteral administration remains a highly regulated route, where all components, both active drug and excipients, are strictly selected and need to be stable and suitable for injection. Primary packaging, which is in contact with the injectable product, must follow many rules given by USP $(<381><659><660>$ $<661><671>$ ) or European Pharmacopeia (21CFR75) regarding specific requirements for container materials and packaging closure. All packaging must also avoid any extractables and leachables [5]. 


\section{The importance of packaging}

The main definitions of packaging

The main purpose of any packaging is to contain, separate and prevent the product from touching or being exposed to the environment. The second purpose is to protect and to prevent the product from sustaining any physical damage [6]. For drug forms, packaging can be classically summarized into three elements: container; case; and notice. Now, the packaging's role is expanded and can include sterility, closing, communication (via the label), compliance, tamper evidence for anticounterfeiting and temperature stability [7]. Primary packaging should use specific materials as they are in direct contact with the drug form. Secondary packaging and the labeling have a huge impact on recognition of the right product (dosage) for a correct intravenous or intramuscular use [7].

\section{Packaging \& guidelines}

Various international guidelines such as ICH Q9 (Quality Risk Management) "where the protection of the patient by managing the risk to quality should be considered of prime importance" [8] or ICH Q8 (Pharmaceutical development) "where in all cases, the product should be designed to meet patients' needs and the intended product performance" [9] have emphasized the effects of packaging, which is much more than a simple container. The Good Practice Guide on risk minimization and prevention of medication errors (EMA/606/103/2014) targets specific populations (children, elderly) and emphasizes this effect of packaging in order to avoid errors [10]. European Parliament (2011/62/EU) has also given rules related to the prevention of falsified medicine, with requirements for the secondary pack of prescribed drugs to have a tamper verification feature [11]. The US FDA has also edited guidance for applying human factors/usability that can help manufacturers improve the design of their devices in order to optimize the ease of use and minimize potential user errors and resulting harm [12].

\section{Counterfeiting, a major problem}

According to statistics given by the WHO, between 7 and 15\% of all medicines sold in developed countries are counterfeit drugs. This problem becomes worse in developing countries, with nearly $10-30 \%$ of all medicines sold that are counterfeit [13]. Several new regulations are being implemented across Europe, Asia and North America to fight counterfeiting drugs throughout the supply chain. As an example, serialization is becoming a strict regulation worldwide and is one active step to protect the consumer. Since 2011, for all prescribed medication, a feature to verify the originality of the pack must be integrated as a tamper-evident system to limit counterfeit [7].

\section{Main economical packaging aspects}

The global packaging market is estimated to reach US\$ 839 billion in 2015 and should reach US\$998 billion in 2020 (with an average annual growth rate of 5\%, it should also reach US\$ 1100 billion in 2024). Regarding packaging materials, rigid plastic should enjoy the strongest growth until 2020 with a compound annual growth rate of $4.4 \%$ until 2020 [14-16]. In 2016, five major elements impacted primary packaging: serialization; the use of glass versus plastic; innovative products; self-administration; and patient compliance [17]. A consumer survey [18] has pointed 'practicality' (50\%), 'ease of use' (45\%) and 'design' (42\%) as the main levers for innovation. The packaging of the future, the 'Millennial Packaging' will be resolutely more ultrasmart (digital packaging, smart label, anticounterfeiting) with greener packaging (bioplastic and biodegradable for sustainability). It is related to the 'Millennial' population (those born between 1980 and 2000) who are the first generation to have grown up with the internet, and who represent a huge target all over the world (80 million in the USA, 364 million in China and in France half of the active population by 2020) [14,15].

\section{Drug design adaptation \& various needs \& users}

\section{Design definition}

The design is definitely not an option, as it is now clearly associated with the product's function, and integrating the design in the development process means creating a product that is functional in all areas of everyday life. By this way, it adds a value to the product and encompasses its ease of use and the product's performance, which are particularly essential for a drug form in the health area [19].

\section{Design \& drug forms}

The Public Health Code defines a drug form as a specific product used to treat a disease, showing specific rules depending on the country and whether it is a prescribed or an 'over the counter' drug. The patient is the real 
final user of medicine, as he/she is placed at the chain end, after the prescriber (who chooses the products) and pharmacist (who delivers them). However, it is fundamental to realize that the patient is not the only user in contact with the drug form that can be (especially for injectable route) administered by practitioners, nurses or caregivers, or sometimes also by patients themselves [20].

Parenteral administration can be involved in emergency use (where the design should fundamentally allow a quick and safe use) as well as for chronic use (where the design should allow ease of use and should be painless) [2]. For these elements, the design appears fundamental for helping toward use and compliance.

\section{Compliance definition}

A compliant patient is one that takes the right medicine at the right time in the right way. It remains a huge challenge (especially for patients with chronic disease) to correctly follow medical advice and a prescription over a long period of time. WHO indicates that $50 \%$ of patients with chronic disease do not comply [21]. Three main pillars are described for helping compliance: knowing what the treatment is (understanding correctly the full elements on use, storage and administration); access to treatment (not only in financial term but also with a proposal of treatment that presents no serious side effect); and last but not the least, the willingness to join the process, because only the patient himself/herself can decide whether to take the treatment (however, nurses or doctor can be involved for injection in an emergency) [20].

\section{Overview on patient diversity}

For young patients, the various scales of ages (from preterms to teenagers) are given by the European Medical Agency $[22,23]$ with the idea that children are not at all 'small adults' and that age remains a key factor "that determines the stage of organ development and physiological functions". Dosage varies throughout infancy and childhood; they are frequently quoted as amount $/ \mathrm{m}^{2}$ or amount $/ \mathrm{kg}$. Injection systems must provide a perfect accuracy - even for a low dosage - and of course, any pain related to needle-use must be avoided or limited [24].

On the other end of the scale, the elderly also represent a huge challenge, for all the drug forms as well as for injectables. The aging population is a universal phenomenon (globally, the number of people over 65 years of age in 2015 is projected to reach $12 \%$ in 2030), and there is a particular increase of people over 80 years of age. This age group remains the top-consumer population and polypharmacy (often observed in this age group) is unfortunately very often associated with drug misuse and poor compliance $[17,20]$. Injectable forms must be adapted to avoid/limit pain and to propose a safe recognition and use of all the systems [14].

From the very young to the elderly, this large diversity of users means various needs such as safety and time-saving are the main requests from nurses and practitioners; painless, ease and safe use remain the most important factors for patients.

\section{Injectable forms, proposals for a better use}

\section{Systems for safe preparation}

Therapeutic agents administered by injection can be proposed in solution, suspension, or emulsion, according to the physical-chemical properties of active drug. Sometimes, medicines must be reconstituted just before the injection. Various transfer mix-sets have been proposed for a safer reconstitution in a closed system. These mix-sets have evolved subsequently to limit the number of steps of preparation, retaining the basic idea: facilitating the reliability of reconstitutions. They appear both secure and time-saving for the healthcare workers and patients themselves (increasingly involved in self-injection with development of chronic pathologies needing regular injections). Most recently, the digital era has been integrated with various automated systems providing a perfect reliability. This is an absolute necessity for these complex, unstable and - most of the time - expensive assets.

\section{Era of 'ready-to-administer'}

Prefilled syringes were invented over 30 years ago by BD Medical Pharmaceutical systems and offer an 'all-in-one' and 'ready-to-administer' system for patients. They are now fully developed and allow both to contain and to administer the injectable forms; they can be used alone or integrated with an auto-injector or pump. Prefilled syringes can be adapted to provide an easier and quicker injection in an emergency; for specific formulations (biotechnologies) the prefilled syringes will limit the material/product contact. 


\section{Injection \& safety of use}

Also of interest, 'tailor-made' design adaptation is given with the safety-needle that appeared in the 2000s. They represent a response to the problem of blood diseases with the request of a needle protection after injection. Active systems require an additional gesture to activate the protection device, whereas passive systems set off automatically once the injection is done. Various innovations can be added and combined: such as prefilled syringes allowing mixing and reconstitution of product with a safety-needle and sometimes a tamper verification feature. Limiting the pain caused by the needle remains a universal request and a great deal of work has been done on new materials and designs to adapt the shape and thickness of the needle for decreasing pain at the injection point.

\section{A world of pen injectors}

More than 350 million patients with Type 1 diabetes need regular insulin injections, leading to a successive generation of pen injectors. Suppliers regularly propose new pen injectors with improvements that can provide safe accuracy for mid-units, offer various ergonomic adaptations for easier use by the elderly fingers and can integrate digital counters for dose memorization. Pen injectors can also help avoid stigmatization, as the pen can look similar to a regular writing pen and can also sometimes be customized for children and teens [25].

\section{Injectable delivery \& the full integration of the digital era}

The digital era is definitely present for injectable systems with safe auto-injectors that can be integrated onto a screen (providing a full explanation for the injection process) and can be connected on an internet platform (allowing a better monitoring of chronic diseases). New connectable, injectable pens (or additional sensors added on conventional injectable pens) can offer a direct digital full service on the injectable target in relation to the internet and apps on disease monitoring [26].

\section{Infusion \& innovative systems}

Infusions are more often used in hospitals, where products need to be both safe but also time-saving. With that target in mind, the number of mix-set devices has multiplied and these ensure a safe mixing of the compounds within sealed containers or protected vials, allowing preparation in advance, which may be useful for nurses. Another innovative system proposed are self-cleaning infusion bags, which allow the medication to be delivered to its last droplet, which is very important in oncology treatment.

\section{Importance of secondary packaging \& labeling}

As previously mentioned, a clear recognition of the dosage and product is fundamental for injectable forms. For this, the product name and dosage should be mentioned in large font (similar colors can be retrieved on labeling and secondary packaging). In many cases (such as with insulin pen injectors) the photo of the drug forms can be directly printed on this to allow the therapeutic education of patients (e.g., seeing the safety-needle). For some injectable forms that must be used in emergency situations, pictograms can be useful for explaining more easily the correct steps to follow. Regarding the large universe of labels, multifunctional labeling is very often proposed for injectable drug forms. Specific labels can integrate fine foam, for offering a protection for the vials and avoiding them being broken, or labels can be designed to cover the entire vial and prevent bottle breakage - a system particularly useful for highly potent drugs.

\section{Conclusion}

This short overview emphasized the highly regulated world of injectable forms, and demonstrates that a great number of clever adaptations and rules do not hamper innovation. Some of these improvements can be used for other administration forms (such as inhaler devices). The digital era has become a reality, fully integrated in all fields as it provides a better monitoring of treatment and the best explanation on chronic disease. A simple add-on solution to record and transmit injection data allows the creation of a long-term health benefit that can decrease the costs of healthcare and introduce a new interface with patients. Despite the large diversity of all these systems, they show common points for helping the use, improving the compliance and ultimately providing proof that packaging remains an essential partner for the proper use of medicines. 
Financial \& competing interests disclosure

The author has no relevant affiliations or financial involvement with any organization or entity with a financial interest in or financial conflict with the subject matter or materials discussed in the manuscript. This includes employment, consultancies, honoraria, stock ownership or options, expert testimony, grants or patents received or pending, or royalties.

No writing assistance was utilized in the production of this manuscript.

\section{References}

1 Research and markets: injectable drug delivery market: by devices, therapeutics and formulations 2015-2020. www.businesswire.com/news/home/20150930006037/en/Research-Markets-Injectable-Drug-Delivery-Market-Devices

2 Gauthier P. Design adaptation to injectable forms depending users and drugs specificities. Presented at: $2^{\text {nd }}$ Annual Prefilled Syringes and Novel Injectors Devices, Informa Drug Delivery Summit. Berlin, Germany 17-18 September 2013.

3 USP 35 NF 30, vol 1, (2012).

4 Pharmacopée Européenne 8.0, (2014).

5 Gauthier P. 'Extractables and leachables, an overview'. Aerosol Eur. 23(6), 7-13, (2015).

6 Bauer EJ. Pharmaceutical Packaging Handbook. Informa 585, (2009).

7 Gauthier P. Much more than packs! Overview of innovative packaging that changes life of drugs forms. Presented at: Pharmapack Europe 2015, Paris, France 11-12 February 2015. 'Packaging as a partner for compliance'. Aerosol Eur. 24(5), 17-21 (2016).

8 ICH Q9 Guideline Q9 on quality risk management, September 2015 EMA/CHMP/ICH/24235/2006. www.ema.europa.eu/docs/en_GB/document_library/Scientific_guideline/2009/09/WC500002873.pdf

9 ICH Q8 (R2) Guideline on Pharmaceutical Development September 2015 EMA/CHMP/ICH/167068/2004. www.ema.europa.eu/docs/en_GB/document_library/Scientific_guideline/2009/09/WC500002872.pdf

10 Good practice on risk minimization and prevention on risk medication errors. www.ema.europa.eu/docs/en_GB/document_library/Regulatory_and_procedural_guideline/2015/11/WC500196981.pdf

11 Directive 2011/62/EU of the European Parliament and of the Council . . . as regards the prevention of the entry into the legal supply chain of falsified medicinal products. http://ec.europa.eu/health/sites/health/files/files/eudralex/vol-1/dir_2011

12 Applying Human factor and usability engineering to medical devices. www.fda.gov/downloads/MedicalDevices/./UCM259760.pdf

13 Health Research Funding.org. http://healthresearchfunding.org/20-shocking-counterfeit-drugs-statistics

14 The Future of Global Packaging 2020, Smithers Pira. www.smitherspira.com/resources/2015/september/insight-four-key-trends-driving-flexible-packaging

15 The Future of Packaging - Long Term Strategic Forecasts to 2024, Smithers Pira. www.smitherspira.com/industry-market-reports/packaging/the-future-of-packaging-design-long-term-strategic

16 Health sector, key figures. www.all4pack.com/Archives/2014/Fiche-marche/Health-sector

17 Global medicines use in 2020: outlook and implications. IMS Institute, dec 2015. Global trends impacting the market for packaging machinery PMMI, 2016.

18 Exclusive findings of the 2016 All4Pack observatory. www.all4pack.com/the-show/Previous-sessions/ALL4PACK-Paris-2016/2016press-releases/Exclusive-findings-of-the-2016-ALL4PACK-Observatory

19 Gauthier P. Formulation and packaging design for NCE, examining specific population and product repositioning. Ther. Deliv. 2(7), 845-848 (2011).

20 Gauthier P. Packaging as pharmaceutical partner: from dream to reality, now with digital integration. Presented at: Pharmapack Europe 2017, Paris, France, 11 February 2017.

21 Adherence to long term therapies: evidence for action. www.who.int/chp/knowledge/publications/adherence_report/en/

22 Committee for Medicinal Product for Human Use (CMPH). Reflection Paper: Formulations of Choice for the Pediatric Population. European Medicines Agency (EMA): London, UK, 28 July 2006; EMEA/CHMP/PEG/194810/2005.

23 European Medicine Agency, 2011. Guideline on Pharmaceutical Development of Medicines for paediatric use. Draft. EMA/CHMP/QWP/180157/2011, London, UK.

24 Gauthier P, Cardot JM. Developing drugs for children and the adjustment of medication - is it a new challenge or an adaptation of past ideas? J. Pers. Med. 1(1), 5-16 (2011).

25 Gauthier P, Cardot JM. Teenagers as a moving target: how can teenagers be encouraged to accept treatment? J. Pers. Med. 2, 277-286 (2012).

26 Gauthier P. Electronic health and the role of software in drug dispensing and administration. Ther. Deliv. 7(10), 659-664 (2016). 



\title{
An industry update: the latest news in therapeutic delivery
}

\author{
Elaine Harris ${ }^{*}, 1$ \\ ${ }^{1}$ Innovation21, Unit B Deansgrange Business Park, Blackrock, Co Dublin, Ireland \\ * Author for correspondence: Tel.: +353 872988 189; Elaine@innovation21.ie
}

The present industry update covers the period 1-31 July 2017. Information was sourced primarily from company press releases, regulatory and patent agencies, scientific literature and various news websites. There was positive approval news this month for GlaxoSmithKline for its new self-injecting treatment for systemic lupus erythematosus but less positive news for Ocular Therapeutix, a new drug application for its treatment for postoperative ocular pain, DEXTENZA ${ }^{\mathrm{TM}}$ was rejected for a second time. Endo Pharmaceuticals agreed to withdraw its opioid formulation Opana ${ }^{\circledR} \mathrm{Er}$ due to abuse concerns. Collaborations for novel therapeutic delivery research were announced this month by Takeda and BioSurfaces, Catelent and Rutgers University, Lilly and Purdue University and Titan Pharmaceuticals with Walter Reed and the Southwest Research Institute. A number of companies announced significant financing deals to allow for the clinical development of products with enhanced delivery options including Sebacia and Diasome.

First draft submitted: 15 August 2017; Accepted for publication: 1 September 2017; Published online: 24 October 2017

Keywords: bioresponsive $\bullet$ competitor intelligence $\bullet$ emerging technologies

\section{Business development}

Takeda, BioSurfaces to collaborate on gastrointestinal drug-delivery devices

Takeda Pharmaceutical Company Ltd and BioSurfaces, Inc. (both MA, USA) announced on 25 July that they have agreed to initiate a research program with the aim of developing novel, innovative medical devices to treat patients with gastrointestinal (GI) diseases. This program will make use of BioSurfaces' proprietary nanomaterial technology which exploits the ability to produce nanofibrous materials with US FDA approved polymers. It is proposed that the BioSurfaces' process will allow for the incorporation of therapeutics for localized delivery to the GI tract. Financial details of the collaborative agreement were not disclosed [1].

Sebacia raises $\$ 36 \mathrm{~m}$ for novel microparticle acne treatment

Sebacia (GA, USA) announced on 25 July that it had completed a Series D funding round and had leveraged additional debt facility from Hercules Partners.

Sebacia's proprietary technology, Sebacia Microparticle ${ }^{\mathrm{TM}}$ is composed of a silica core encapsulated in a gold coating and is designed to target the light from laser systems typically used by dermatologists. This targeted treatment aims to photothermally affect change in the sebaceous glands and thus treat acne.

The company announced that the finance raised would be used to complete US pivotal trials with results expected by Q3 2018. The technology is already CE marked in Europe and approximately 2000 procedures have been performed to date [2].

\section{Gurnet Point acquire Innocoll}

Athlone (Ireland)-based Innocoll announced on 24 July the completion of its acquisition by Gurnet Point through its wholly owned subsidiary Lough Ree Technologies Ltd. Announcing the acquisition Christopher Viehbacher, Managing Partner of Gurnet Point Capital commented, "Gurnet Point is pleased to announce that its acquisition of Innocoll is now effective. We are excited by the prospect of helping to develop and bring XaraColl to the market and we look forward to assisting with the re-submission of Xara Coll for FDA approval and preparing for its commercialization". 
XaraColl is Innocoll's lead product - a bupivacaine $\mathrm{HCl}$ collagen-matrix implant in development for the treatment of postsurgical pain. In December 2016 received a 'Refusal to File' letter from the FDA for the New Drug Application (NDA) submitted for XaraColl. Following further meetings with the FDA, the company indicated that it intended to address certain issues raised in the Refusal to Fle by conducting an additional short-term pharmacokinetic study and several short-term nonclinical toxicology and biocompatibility studies. Gurnet Point now believes that these studies, if successful, may be completed in time for a resubmission of the NDA at the end of 2017 [3].

\section{Diasome lands $\$ 30 \mathrm{~m}$ to fund clinical trials of liver-targeted insulin}

Diasome (OH, USA) announced on July 6 that it had concluded an agreement that allowed it to access of over $\$ 30 \mathrm{~m}$ to fund the development of their liver-targeted insulin product. Medicxi, a European life sciences venture capital firm is the lead investor in this round of funding. Diasome's proprietary technology for targeting insulin to the liver is known as hepatocyte directed vesicles (HDV) and it preferentially allows insulin to be delivered to liver hepatocytes. The funding achieved will allow Diasome to progress their lead candidate, HDV-Insulin, through clinical trials. The HDV-insulin therapy is specifically designed to mimic the mealtime exposure of the liver to insulin and will include options for the treatment of both Type 1 and Type 2 diabetes (T1D and T2D), respectively.

Significant funding was provided from the JDRF T1D Fund, a Boston-based venture philanthropy fund dedicated to facilitating solutions to treat, prevent and cure T1D $[4,5]$.

\section{Catalent \& Rutgers partner to study pediatric drug formulation \& delivery}

The Catalent Applied Drug Delivery Institute (NJ, USA) and Rutgers University (NJ, USA) announced on 20 July that they had initiated a partnership to identify diseases and therapies for which there is significant need for novel pediatric formulations.

The Catalent Applied Drug Delivery Institute was established in 2012 with an aim to accelerate the use of advanced formulations, dosage forms and delivery devices.

According to Dr. Cornell Stamoran, Catalent's VP Strategy, "Most initiatives focus on individual new drugs or disease areas - we're collaborating to identify a broad platform, founded on rigorous stakeholder insights, to establish a foundation for future pediatric drug development" [6].

\section{Inovio looks to raise $\$ 75 \mathrm{~m}$ in public offering}

On 18 July, Inovio (PA, USA) announced that it was going to offer $\$ 75.0$ million of shares of its common stock in an underwritten public offering. Inovio intends that the proceeds from this offering be used for such purposes as, clinical trial expenses, research and development expenses, manufacturing expenses and other business development activities.

Inovio's therapeutic focus is the delivery of DNA-based immunotherapeutic with lead candidates in the area of cancers caused by the human papilloma virus, their proprietary electroporation technology facilitates delivery of the DNA vaccines to different target tissues [7].

\section{Titan Pharmaceuticals partners with Walter Reed \& the Southwest Research Institute for long-acting antimalarial implant}

On 6 July, the Californian-based Titan Pharmaceuticals announced a collaboration with the Walter Reed Army Institute of Research (WRAIR) and Southwest Research Institute (SwRI) to evaluate ProNeura ${ }^{\mathrm{TM}}$, Titan's proprietary long-term, continuous drug delivery technology, for prevention of malaria.

ProNeura is a subdermal implant that can offer continuous drug release and nonfluctuating medication levels over a period of 3 months to a year. In 2016, the FDA approved Titan's first product employing this technology for the long-term maintenance treatment of opioid dependency, Probuphine ${ }^{\circledR}[8]$.

\section{Eli Lilly \& Purdue University announce strategic research collaboration}

On 6 July, Purdue University and Eli Lilly (both IN, USA) announced a new \$52 million strategic research collaboration in the areas of improving the delivery of injectable medicines and also developing novel predictive models for clinical success that can reduce the risks associated with drug development and more effectively predict the outcome of new therapies.

This new agreement has a term of 5 years and builds upon the previous successful relationship between the company and the university and is Purdue's largest strategic collaboration with a single company [9]. 


\section{pSivida licenses European rights for Durasert to Alimera}

The Massachusetts-based specialty drug delivery company pSivida announced on 10 July that it had agreed to license European, Middle East and African rights for its product Durasert ${ }^{\mathrm{TM}}$ for the treatment of posterior segment uveitis in an amendment to an existing global collaboration agreement with Alimera (GA, USA).

Under the new agreement, pSivida will withdraw its centralized EU application for the drug's indication for posterior uveitis and allow Alimera to file an application for the indication in the countries where it is currently approved for Diabetic Macular Edema [10].

\section{Developments \& clinical trials}

\section{Submission of an NDA for opioid disorder by Camarus}

Swedish company, Camarus, along with its co-development partner Braeburn Pharmaceuticals (NJ, USA) announced on 20 July that they had submitted an NDA to the FDA for their depot formulation of buprenorphine (CAM2038) for the treatment of opioid use disorder.

Camurus' proprietary technology FluidCrystal ${ }^{\circledR}$ is a drug delivery technology that is based on endogenous polar lipids that can spontaneously form liquid crystal nanostructures in aqueous environments. These lipid structures can then carry very high drug payloads and are suitable for formulation as intravenous bolus injections, infusion products and subcutaneous injection products.

Data from 944 study participants across seven Phase II/III clinical trials was submitted as part of the NDA submission for CAM2038 [11].

\section{FDA grants priority review to Kaleo's auto-injector for children}

The Virginia-based device company Kaléo announced on 27 July that the FDA had a Priority Review of its supplemental NDA for its AUVI-Q $0.1 \mathrm{mg}$ product. This is the first epinephrine auto-injector specifically designed for the treatment of anaphylaxis in infants and small children weighing 16.5-33 pounds. The design of the device accommodates both a shorter needle length and lower dose than other marketed epinephrine auto-injectors [12].

\section{Surmodics to launch pivotal trial for drug-coated balloon}

Surmodics (MN, USA) a company that specializes in surface modification technologies for intravascular medical devices announced on 26 July that it had received an investigational device exemption from the FDA to initiate a pivotal clinical trial of its SurVeil ${ }^{\mathrm{TM}}$ drug-coated balloon (DCB). This new DCB incorporates new proprietary coating for interventional treatment. This trial will evaluate the safety and effectiveness of the device for treatment of subjects with symptomatic peripheral artery disease due to stenosis of the femoral and/or popliteal arteries and will enroll up to 446 subjects at 80 sites in the USA and elsewhere [13].

\section{Samumed granted orphan drug status for inhaled idiopathic pulmonary fibrosis therapy}

Samumed (CA, USA) announced on 25 July that it had been granted orphan drug status for its SM04646, to treat idiopathic pulmonary fibrosis (IPF). Development of the therapeutic as an inhalable, nebulized formulation is ongoing. IPF is incurable and current treatment options are very limited. IPF is the most common interstitial lung disease in USA, with an estimated prevalence of 13.2-27.9 per 100,000 women and 20.2-63.0 per 100,000 men. After diagnosis, the estimated median survival for IPF patients is between 2.5 and 3.5 years [14].

Mylan, Theravance's inhaled chronic obstructive pulmonary disease therapy: positive late stage clinical trial results announced

Mylan (Dublin, Ireland) and Theravance (Hertfordshire, UK) announced on 19 July positive results for the Phase III study of revefenacin (TD-4208) in patients with chronic obstructive pulmonary disease (COPD).

TD-4208 is an investigational long-acting muscarinic antagonist and a proposed once-daily, nebulized bronchodilator for the treatment of COPD. Theravance Biopharma and Mylan have established a strategic collaboration to develop and commercialize nebulized revefenacin products for COPD and other respiratory diseases. TD-4208 is delivered via Mylan's dry-powder drug-delivery platform technology. TD-4208 was acquired from Theravance in 2015 by Mylan in a $\$ 265$ million deal [15]. 


\section{Aequus to launch trial for anti-nausea patch}

The Vancouver-based Canadian company Aequus announced on 5 July that it had received approval from Health Canada to initiate a proof of concept trial for its novel anti-nausea patch, AQS1303. The patch contains a combination of pyridoxine and doxylamine and it is hoped that it will offer a more convenient option for patients who currently receive the medication in the oral form up to four-times daily. The company also announced that in parallel to the Canadian Proof of Concept study it will also prepare for pre-Investigational New Drug (pre-IND) meeting with the FDA to define the clinical strategy for regulatory approval in the USA [16].

\section{AntriaBio commences Phase I studies for their insulin product}

It was announced on 6 July that AntriaBio (CO, USA) had commenced its Phase I first-in-human clinical trial of its lead product candidate, AB101. This initial study is a single ascending dose study to assess the safety and tolerability, pharmacokinetics and pharmacodynamics of AB101 in patients with Type 1 diabetes mellitus. The company aims for this trial to facilitate the demonstration of clinical proof of concept for AB101 as a onceweekly insulin therapy. AntriaBio's sustained delivery technology is based on poly-lactide co-glycolic acid (PLGA) microspheres with encapsulated Peg $(5 \mathrm{kDa})$-insulin [17].

\section{Biohaven commences Phase III pivotal trials for migraine treatment}

The dates 24 and 31 July saw two announcements from Connecticut's Biohaven on their Phase III studies for the treatment of migraine with their oral rimegepant formulations. Rimegepant is one of a family of calcitonin generelated peptide receptor antagonists under clinical evaluation by Biohaven for a number of indications including migraine and was licensed exclusively from Bristol-Myers Squibb Company [18,19].

\section{Early stage development}

Researchers create unique thermal ablation system to enable transdermal protein delivery

It was reported on 27 July on the website www.news-medical.net that Japanese scientists from the University of Kumamoto are researching a novel thermal ablation system to enable the transdermal delivery of therapeutic proteins. To facilitate the transdermal delivery while minimizing damage to the skin, the researchers used a combination of transparent gel patches, gold nano-rods and near-infrared light. In initial in vivo and in vitro tests with murine skin, the researchers discovered that the near-infrared-irradiated gold nano-rod patches heated the skin to approximately $43^{\circ} \mathrm{C}$ and the fluorescently labeled ovalbumin (FITC-OVA) they used as model therapeutics were significantly translocated. Although this work is at a very early stage of development, the research group is confident that in time, they can advance the current state of the art in transdermal delivery [20].

\section{Hydrogel-based capsules may help patients' compliance}

An article published in 25 July edition of the MIT News (http://news.mit.edu/) reported a paper published in Nature Communications by researchers MIT's Koch Institute for Integrative Cancer Research group on triggerable tough hydrogels (TTH). The group has been working with the Bill and Melinda Gates Foundation to develop ultra-long-lasting capsules, which might last for the entire course of a treatment, or could be taken once a week or once a month, depending on the device. Initial preclinical studies, including large animal studies, have indicated that capsules made from the novel hydrogels may have the capacity to deliver therapeutics over a significant time (days, even weeks). The hydrogels used are manufactured from a combination of polymer networks, one is alginate the other is a polyacrylamide, and these give additional strength and durability to the device/capsule. However, the scientists have also included crosslinking features within these intertwined networks which can be dissolved on demand using biocompatible trigger compounds and this allows for rapid removal and/or dissolution of the device should it become necessary [21].

\section{Preclinical studies on oral monoclonal antibody for autoimmune diseases}

Tiziana Life Sciences (London, UK) published an article in the Journal Clinical Immunology on 28 July that described the results of preclinical studies for Foralumab (NI-0401). Tiziana claim that these studies on the orally available, fully human anti-CD3 monoclonal antibody $(\mathrm{mAb})$ demonstrated prevention of skin xenograft rejection in humanized mice. Until recently, it was thought that oral delivery of mAbs was not achievable. According to Dr Gabriele Cerrone, Chairman of Tiziana Life Sciences, "New data demonstrating oral efficacy in animals with 
foralumab is a major milestone and opens a novel avenue for the treatment of NASH and autoimmune diseases with fully human mAbs" [22,23].

\section{Antibiotic-releasing polymer may help eradicate joint implant infection}

On 18 July, a team of investigators from Massachusetts General Hospital discussed their recently published article in the Journal Nature Biomedical Engineering. The paper describes the development of a novel antibiotic-releasing polymer for the treatment of prosthetic joint infection. Using mathematical and statistical models, researchers developed polyethylene materials which 'doped' with antibiotic clusters. These clusters were designed to release the antibiotic over extended periods of time without compromising the strength of the material. Preclinical models of prosthetic joint infection were produced either by injecting a Staphylococcus aureus-containing solution into the prosthesis or implanting a titanium rod covered with a $S$. aureus biofilm. When the novel antibiotic/polymer matrices were tested, the antibiotic-releasing polymer successfully eliminated the infection. As a control, the antibiotic was also incorporated into the bone-cement spacer but this was not effective in treating the infection [24].

\section{Molecular hitchhiker on human protein signals tumors to self-destruct}

Researchers from Vanderbilt University (TN, USA) have reported, in a paper published in the Proceedings of the National Academy of Sciences USA, that by making slight modifications to a small-interfering RNA molecule, called siRNA-L2, it can effectively 'hitchhike' on the human protein albumin. The ability of this modified siRNA molecule to bind to the albumin allows it to circulate around the body for a number of days - much longer than typically is observable with siRNA-loaded micro/nanoparticles. The authors propose that this mechanism may be used to develop more successful tumor-targeting therapeutics. Initial in vitro results were encouraging - looking at the siRNA-L2 in human breast tumor tissue removed from a donor, the modified molecule appeared to be more effective in targeting the tumor, with siRNA-L2 more than three-times as present in the tumor than siRNA delivered with synthetic nanoparticles [25].

\section{Researchers develop light-sensitive hydrogel for drug delivery}

A report published on the EurekaAlert website on 6 July reported that Hong Kong based-researchers from the University of Science and Technology had developed a B12-dependent light-sensing hydrogel. The hydrogel was created by covalently attaching the photoreceptor C-terminal adenosylcobalamin binding domain (CarHC) proteins. The researchers claim that the hydrogels created via this process allow for the design of 'smart' materials. It is proposed that these photoresponsive hydrogels would provide 'tuneable' drug-delivery opportunities as they undergo a rapid gel-sol transition caused by light-induced CarHC disassembly. It is claimed that these novel hydrogels would be particularly suitable for the delivery of stem cells and protein molecules. The initial article describing this work was published in the 6 June edition of the Proceedings of the National Academy of Sciences USA $[26,27]$.

\section{Defymed reveals development of novel insulin delivery device}

The Strasbourg-based company Defymed announced its novel delivery device for insulin for the treatment of Type 1 diabetes, ExOlin ${ }^{\circledR}$ on 10 July.

ExOlin is a novel medical device under development by Defymed, the company claims that if it proves successful in clinical trials, it will allow for instant administration of an intraperitoneal injection of insulin using a simple subcutaneous injection (via an external connection to a syringe, pen, insulin pump, etc.). The company aims to enter clinical trials in 2018 predicts that the product could be commercially available by the end of 2020 after obtaining CE marking [28].

\section{Regulatory news \& approvals}

\section{Endos' Opana ${ }^{\circledR} E R$ removed from market}

Endo Pharmaceuticals (Dublin, Ireland) announced on 6 July that following consultation with the FDA, it had decided to voluntarily remove OPANA ${ }^{\circledR}$ ER from the market. This move follows on from a request by the FDA in June 2017 that Endo remove its opioid pain medication Opana ER from the market due to risks of abuse. The company was very keen to reiterate that the withdrawal did not reflect a finding that the product was not safe or effective when taken as prescribed. As a result of the decision to remove the product from market, the company 
indicated that it expected to incur a pretax impairment charge of approximately $\$ 20$ million in the Q2 of 2017 to write-off the remaining net book value of its Opana ER intangible asset [29,30].

GlaxoSmithKline receives FDA approval for a self-injectable formulation of belimumab for systemic lupus erythematosus

GlaxoSmithKline (London, UK) announced on 20 July that the FDA has approved Benlysta - a self-injectable formulation for systemic lupus erythematosus (SLE). Benlysta is the first subcutaneous self-injection treatment option approved for patients with SLE. SLE is the most common form of lupus. It is a chronic, incurable autoimmune disease which produces autoantibodies that can attack almost any system in the body. This product will be the second formulation of belimumab and it complements the existing intravenous formulation which was approved in 2011. It is expected that the product will be commercially available by the end of August 2017.

Belimumab is a human $\mathrm{mAb}$ that inhibits B-cell activating factor (also known as B-lymphocyte stimulator [BLyS]). Belimumab was developed by Human Genome Sciences and Cambridge Antibody Technology. GlaxoSmithKline acquired Human Genome Sciences, took belimumab through Phase III clinical trials and markets belimumab [31].

\section{Spectranetics receives approval from the FDA for Stellarex DCB}

Spectranetics (CO, USA) and medical device company that were recently acquired by the Dutch Royal Philips company, announced on 26 July that it had received premarket approval of its Stellarex ${ }^{\mathrm{TM}}$ DCB. Stellarex DCB was designed to restore and maintain blood flow to the superficial femoral and popliteal arteries in patients with peripheral arterial disease. Clinical trial data obtained during the pivotal studies indicated that it was successful in treating patients with low paclitaxel drug dose. According to Dr Sean Lyden the co-principal investigator of the study "When considering options, I think most clinicians would prefer to use a lower drug dose if they can also achieve great patency results" [32].

\section{FDA rejects Ocular's Dextenza for a second time}

Ocular Therapeutix ${ }^{\mathrm{TM}}$ (MA, USA) disclosed on 11 July that it had received a complete response letter (CRL) from the FDA, regarding its resubmission of an NDA for DEXTENZA ${ }^{\mathrm{TM}}$ (dexamethasone insert) $0.4 \mathrm{mg}$ for the treatment of ocular pain following ophthalmic surgery. The CRL stated that the FDA has determined that it cannot approve the NDA in its present form. This was the second submission of the NDA for the company. In the CRL, deficiencies observed during a pre-NDA approval inspection in May 2017 in manufacturing processes and analytical testing related to manufacture of drug product for commercial production were disclosed. DEXTENZA was designed to deliver dexamethasone to the ocular surface for up to 30 days for the treatment of postocular surgery associated pain. According to the company's President and CEO, Amar Sawhney, the company hopes these issues can be resolved and will allow for DEXTENZA to be approved in the future [33].

\section{Patents}

Biohaven (CT, USA) announced on 13 July that it had received a notice of allowance from the US Patent and Trademark Office (USPTO) for its patent application number 14/385551. This patent application covers claims that concern prodrugs of riluzole - a glutamate modulating agent. These prodrugs are being developed by Biohaven for the treatment of obsessive compulsive disorder, anxiety disorders and cancers [34].

\section{Financial \& competing interests disclosure}

E Harris is a partner in Innovation21 Ltd, providing consultancy, advisory and evaluation services to pharmaceutical/biotechnology companies and government funded agencies.

The author has no other relevant affiliations or financial involvement with any organization or entity with a financial interest in or financial conflict with the subject matter or materials discussed in the manuscript apart from those disclosed.

No writing assistance was utilized in the production of this manuscript.

\section{References}

1 Takeda and BioSurfaces announce joint research program to explore promising devices to treat gastrointestinal diseases. www.takeda.com/newsroom/newsreleases/2017/takeda-and-biosurfaces-announce-joint-research-programto-explore-promisingdevices-to-treat-gastrointestinal-diseases/ 
2 Sebacia announces $\$ 36$ million equity and debt financing to advance breakthrough in-office procedure for acne. www.sebacia.com/media/SebaciaPressRelease20170725.pdf

3 Innocoll and Gurnet Point announcement relating to the scheme becoming effective. www.innocoll.com/investors.aspx?NEWS@http://phx.corporate-ir.net/phoenix.zhtml?c=253636\&p=irol-news\&nyo=0

4 Diasome Pharmaceuticals, Inc. Receives funding led by Medicxi. www.businesswire.com/news/home/20170705005155/en/Diasome-Pharmaceuticals-Receives-Funding-Led-Medicxi

Diasome Pharmaceuticals, Inc. Receives funding from JDRF T1D fund. www.jdrf.org/press-releases/diasome-pharmaceuticals-inc-receives-funding-jdrf-t1d-fund/

6 Catalent Applied Drug Delivery Institute partners with Rutgers University to better understand the challenges of pediatric drug formulation and delivery. www.catalent.com/index.php/news-events/news/Catalent-Applied-Drug-Delivery-Institute-Partners-withRutgers-University-to-Better-Understand-the-Challenges-of-Pediatric-Drug-Formulation-and-Delivery

7 Inovio announces proposed public offering of common stock. http://ir.inovio.com/news/news-releases/news-releases-details/2017/ Inovio-Announces-Proposed-Public-Offering-of-Common-Stock/default.aspx

8 Titan Pharmaceuticals and Walter Reed Army Institute of Research collaborate to evaluate proneura antimalarial implants. www.titanpharm.com/news/press-releases/detail/174/titan-pharmaceuticals-and-walter-reed-army-institute-of

9 Lilly and Purdue University announce strategic research collaboration. www.purdue.edu/newsroom/releases/2017/Q3/lilly-and-purdue-university-announce-strategic-research-collaboration.html

10 pSivida out-licenses EMEA Rights for Durasert ${ }^{\mathrm{TM}}$ three-year treatment for posterior segment uveitis while retaining U.S. Commercial Rights; amended global collaboration agreement with Alimera for ILUVIEN ${ }^{\circledR}$ improves pSivida's revenue generation. http://investors.psivida.com/releasedetail.cfm?ReleaseID=1032622

11 Camurus and Braeburn announce submission of NDA for long-acting Buprenorphine (CAM2038) for Opioid use disorder. www.camurus.com

12 U.S. FDA grants priority review for an epinephrine auto-injector in development by Kaléo specifically for infants and small children. https://kaleopharma.com/u-s-fda-grants-priority-review-for-an-epinephrine-auto-injector-in-development-by-kaleo-specifically-forinfants-and-small-children/

13 Surmodics to launch pivotal trial for drug-coated balloon. www.drugdeliverybusiness.com/surmodics-launch-pivotal-trial-drug-coated-balloon/

14 FDA grants orphan drug designation to Samumed's investigational SM04646 for IPF treatment. www.samumed.com/medium/image/ samumed-receives-orphan-drug-designation-from-fda-for-sm04646-as-a-treatment-for-idiopathic-pulmonar_141/view.aspx

15 Theravance Biopharma and Mylan announce positive results from 12-Month Phase 3 safety study of Revefenacin (TD-4208) in patients with chronic obstructive pulmonary disease (COPD).

http://newsroom.mylan.com/2017--07--19-Theravance-Biopharma-and-Mylan-Announce-Positive-Results-from-12-Month-Phase-3Safety-Study-of-Revefenacin-TD-4208-in-Patients-with-Chronic-Obstructive-Pulmonary-Disease-COPD

16 Aequus receives approval from Health Canada to initiate proof of concept clinical trial of anti-nausea patch. www.aequuspharma.ca/ investors/press-release/aequus-receives-approval-from-health-canada-to-initiate-proof-of-concept-clinical-trial-of-anti-naus

17 AntriaBio announces first patient dosed in Phase 1 clinical study of AB101. www.antriabio.com/news/press-releases/detail/240/antriabio-announces-first-patient-dosed-in-phase-1-clinical

18 Biohaven enrolls first patient in pivotal Phase 3 clinical trial of oral rimegepant for the acute treatment of migraine. http://biohavenpharma.com/biohaven-enrolls-first-patient-in-pivotal-phase-3-clinical-trial-of-oral-rimegepant-for-the-acutetreatment-of-migraine

19 Biohaven initiates second pivotal Phase 3 clinical trial of oral CGRP antagonist for the acute treatment of migraine. http://biohavenpharma.com/biohaven-initiates-second-pivotal-phase-3-clinical-trial-of-oral-cgrp-antagonist-for-the-acute-treatmentof-migraine

20 Researchers create unique thermal ablation system for transdermal drug delivery. www.news-medical.net/news/20170727/Researchers-create-unique-thermal-ablation-system-for-transdermal-drug-delivery.aspx

21 Ingestible drug-delivery materials may help patients comply with treatment regimens. http://news.mit.edu/2017/ingestible-drug-delivery-hydrogel-capsules-0725

22 Publication of research article on Foralumab, a fully human anti-Cd3 antibody being developed as an oral therapy for NASH and autoimmune diseases. www.biospace.com/News/tiziana-life-sciences-plc-release-publication-of/464466/source=MoreNews

23 Ogura M, Deng S, Preston-Hurlburt P et al. Oral treatment with foralumab, a fully human anti-CD3 monoclonal antibody, prevents skin xenograft rejection in humanized mice. Clin. Immunol. pii: S1521-S6616(17), 30342-X (2017). doi:10.1016/j.clim.2017.07.005 [Epub ahead of print].

24 New antibiotic-releasing polymer may simplify treatment of prosthetic joint infection. www.massgeneral.org/about/pressrelease. aspx?id=2124

25 Molecular hitchhiker on human protein signals tumors to self-destruct. www.eurekalert.org/pub_releases/2017--07/vu-mho072117.php 
26 Photo-responsive protein hydrogels as agent for controlled stem cell/protein release. www.eurekalert.org/pub_releases/2017--07/hkuo-pph070617.php

27 B12-dependent photoresponsive protein hydrogels for controlled stem cell/protein release. www.pnas.org/content/114/23/5912

28 Defymed is developing ExOlin ${ }^{\circledR}$, a new insulin delivery device. www.businesswire.com/news/home/20170710005895/en/Defymed-Developing-ExOlin\%C2\%AE-New-Insulin-Delivery-Device

29 Endo provides update on Opana ER. www.endo.com/news-events/press-releases

30 FDA requests removal of Opana ER for risks related to abuse. www.fda.gov/newsevents/newsroom/pressannouncements/ucm562401.htm

31 GSK receives FDA Approval for a new self-injectable formulation of Benlysta (belimumab) for systemic lupus erythematosus. www.drugs.com/newdrugs/gsk-receives-fda-approval-new-self-injectable-formulation-benlysta-belimumab-systemic-lupus-4563.html

32 Spectranetics announces FDA approval of Stellarex ${ }^{\mathrm{TM}}$ drug-coated balloon. www.spectranetics.com/investor-relations/news-release

33 Ocular Therapeutix ${ }^{\mathrm{TM}}$ receives complete response letter from FDA for DEXTENZA ${ }^{\mathrm{TM}}$ NDA. http://investors.ocutx.com/phoenix.zhtml?c=253650\&p=irol-newsArticle\&ID=2285862

34 Biohaven receives notice of allowance from U.S. Patent and Trademark office on patent application for glutamate modulating prodrugs. http://biohavenpharma.com/biohaven-receives-notice-of-allowance-from-u-s-patent-and-trademark-office-on-patent-application-forglutamate-modulating-prodrugs 


\title{
Delivering natural products and biotherapeutics to improve drug efficacy
}

\author{
Mohammad A. Obeid ${ }^{1,2}$, Mohammed M. Al Qaraghuli ${ }^{1}, 3$, Manal Alsaadi ${ }^{1}$, Abdullah R. \\ Alzahrani ${ }^{1,4}$, Kanidta Niwasabutra ${ }^{1}$ \& Valerie A. Ferro ${ }^{*}, 1$ \\ ${ }^{1}$ Strathclyde Institute of Pharmacy \& Biomedical Sciences, University of Strathclyde, 161 Cathedral Street, Glasgow, G4 ORE, UK \\ ${ }^{2}$ Faculty of Pharmacy, Yarmouk University, Irbid, Jordan \\ ${ }^{3}$ Department of Chemical \& Process Engineering, University of Strathclyde, 75 Montrose Street, Glasgow G1 1XJ, UK \\ ${ }^{4}$ Department of Pharmacology \& Toxicology, Faculty of Medicine, Umm Al-Qura University, Makkah, Saudi Arabia \\ * Author for correspondence: v.a.ferro@strath.ac.uk
}

Due to the increasing problem of drug resistance, new and improved medicines are required. Natural products and biotherapeutics offer a vast resource for new drugs; however, challenges, including the cost and time taken for traditional drug discovery processes and the subsequent lack of investment from the pharmaceutical industry, are associated with these areas. New techniques are producing compounds with appropriate activity at a faster rate. While the formulation of these combined with drug-delivery systems offers a promising approach for expanding the drug developments available to modern medicine. Here, various classes of drug-delivery systems are described and the advantages they bring to small molecule and biotherapeutic targeting are highlighted. This is an attractive approach to the pharmaceutical industry and the rising trend in research in this area is examined in brief.

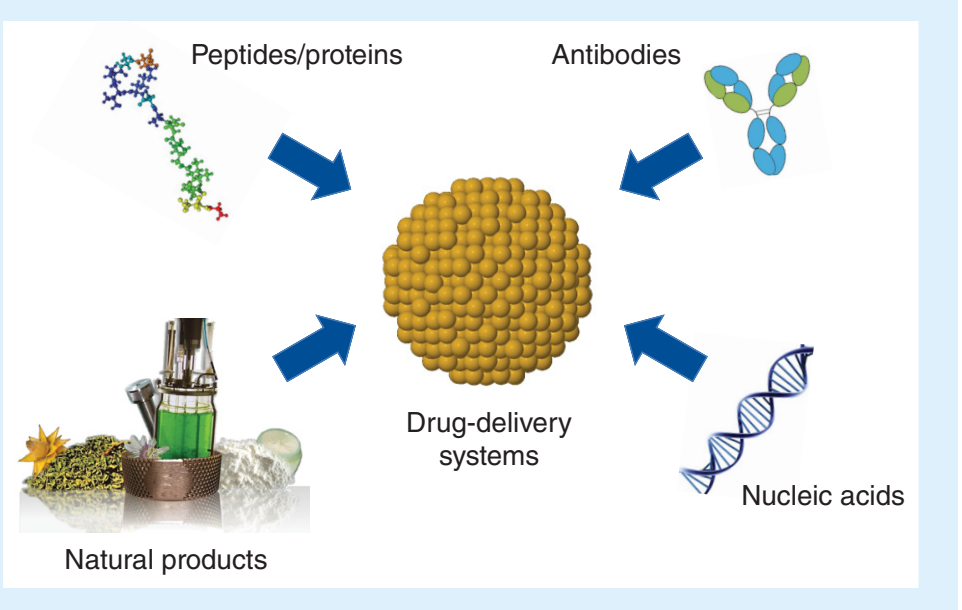

First draft submitted: 19 May 2017; Accepted for publication: 31 August 2017; Published online: 24 October 2017

Keywords biotherapeutics $\bullet$ drug-delivery systems $\bullet$ drug discovery $\bullet$ natural products

New medicines are constantly being developed or repurposed, aimed at curing or preventing diseases or conditions where therapeutic product availability is lacking, or to reduce side effects, improve quality of life, reduce the burden on the cost of healthcare systems, while significantly extending patients' lives. However, drug discovery, research and development $(\mathrm{R} \& \mathrm{D})$ can be an extensive process lasting over $7-10$ years, with an average cost of $\$ 2.6$ billion for each successful drug that reaches the market [1]. These substantial cost and time factors originate from the scientific, technical and regulatory challenges that are needed to fully understand the drug mechanisms of action and physiological interactions for complex diseases at molecular level. Achieving viable commercial success subsequently 
requires investment in highly sophisticated technologies, advanced manufacturing processes and innovative research approaches to tackle the ever-growing cost and time of the entire process.

Traditional medicine has been around for millennia, and in recent decades its use has increased in developing countries, while pharmaceutical companies have embraced synthetic and combinatorial technologies in favor of drug discovery programs based on natural products. However, there is a need for new medicines since growing drug resistance has rendered antibiotics virtually useless [2] and modern medicine is facing a crisis. One hurdle that slows down progress to the market of naturally derived candidate molecules arises when high-throughput screening reveals potentially excellent in vitro therapeutic properties that are then shown to be inactive, toxic or nonselective when evaluated in vivo or in the clinic [3]. One approach to overcome this is to improve the bioavailability and effects of these compounds, or reduce their toxicities by loading them into different types of delivery systems [4]. These have the ability to deliver the therapeutic agent to a particular site of the body at a specific rate and have the potential to enable multiple molecules with different roles to be included in a single delivery system [5]. Our research group has extensive experience in natural products as well as developing drug-delivery systems [6,7]. More recently, we have expanded our research to include biotherapeutic developments [8,9], and in this paper we discuss how these research fields have benefitted from being combined to meet the need to produce new and better drugs. In addition, there is evidence that delivery systems can help to reduce drug resistance and so have a critical role to expand the arsenal of therapies available to modern medicine.

\section{Drug-delivery systems}

Drug delivery refers to the use of a delivery tool or vehicle to carry a therapeutic agent and release it at a specific rate at a particular location [5]. Recently, there has been a significant rise in the use of delivery systems to deliver therapeutic agents for treatment of various diseases with many successful outcomes [10]. Drug-delivery systems can be used to facilitate the delivery of small compounds as well as large molecules such as peptides, nucleic acids, polymers and poorly water-soluble therapeutic agents from natural or synthetic sources [11]. Different types of delivery systems have been effectively used (Figure 1). These include: lipid-based nanoparticles such as liposomes, solid-lipid particles, micelles and niosomes; polymeric nanoparticles such as chitosan and atelocollagen; dendrimers; inorganic nanoparticles such as carbon nanotubes, metal-based nanoparticles, quantum dots and silica nanoparticles [12]. Polymeric nanoparticles (such as poly(lactic-co-glycolic acid), polyethylene glycol, polyvinyl alcohol, poly-l-lactic acid, polycaprolactone and chitosan) and liposomes have been the most tested in combination with natural products. The former are the most commonly used due to their biocompatibility, biodegradability and the ease by which they can be functionalized [13]. All these delivery systems can be characterized in terms of particle size, size distribution, surface charge, shape, stability and encapsulation efficiency [14].

Despite the advantages of the use of delivery systems in developing new medicines, some challenges still need to be addressed before their wide application becomes commonplace; these are related to the fast elimination of the delivery system by the reticuloendothelial system, especially for charged molecules [15]. In addition, toxicity and inflammation as a result of the use of specific types of delivery systems can cause tissue damage [16]. It is also critical for the delivery system to be well established and to meet regulatory considerations given to the manufacturing processes involved, such as physicochemical characterization, controlled drug release, stability, storage, large-scale production and manufacturing costs [17].

\section{Impact of drug-delivery systems in natural product research}

The plant kingdom provides an abundant source of natural products, which has fueled the drug-discovery process and resulted in a plethora of small drug-like molecules to complex polymers [18,19]. Other natural sources include marine organisms, fungi, microbes and invertebrates (such as insects and reptiles) [20]. Between the years 20002006, the natural products field was estimated to produce or be involved in $R \& D$ of approximately $50 \%$ of all small drug molecules, and 10 out of the 44 approved small molecules by the regulatory authorities in 2014 were derived from natural sources [21,22]. Conventionally, natural products are extracted from source materials, concentrated, fractionated and purified. At the early stages of research, a range of techniques are used to isolate pure natural products, and the pros and cons of these have been comprehensively reviewed elsewhere [18,23,24]. Soxhlet solvent extraction, maceration or infusions are utilized to extract constituents. This is usually followed by TLC and NMR analysis for chemical structure elucidation, and MS as a confirmatory technique; these are examples of the core techniques that are usually utilized [7,25], along with open-column chromatography (gel filtration and vacuum liquid separation). For further separation and isolation of pure compounds, modern chromatography 
Figure 1. Different types of delivery system, suitable for use with natural products and biotherapeutics.

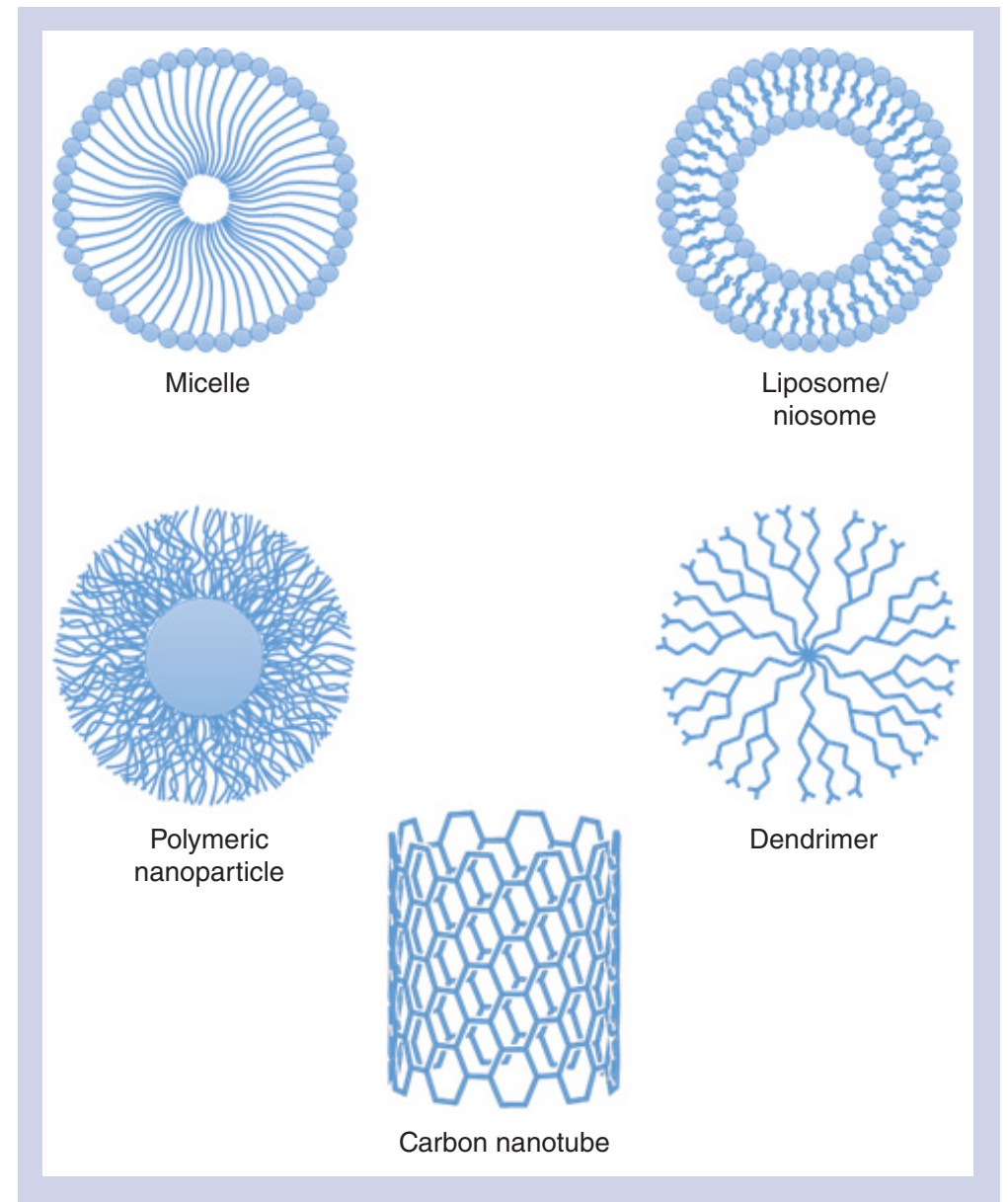

(medium-pressure and high-pressure liquid chromatography systems) is used. In order to identify a particular compound's location within tissues, techniques such as MALDI-TOF MS are usually employed. Compounds isolated in this way from different natural sources have consistently shown promising therapeutic potential against a range of diseases including cancer, hypertension, diabetes and infections [26]. In evaluating extracts and isolated compounds, a range of bioassays are employed to determine the potential therapeutic activity present [27]. For extensive evaluation of mechanisms of action, new technologies such as molecular biology (polymerase chain reaction and RNA-sequencing) have been introduced into this field. These latter methods can show which genes are affected and therefore aid in tailoring the subsequent research toward specific disease pathways. Metabolomics is also used to advance the understanding and development of any potential lead molecules [28].

Use of advanced techniques in natural product research has increased the rate of identification of bioactive compounds, however, this has not translated into an increase in marketable products. The reasons for this are diverse. Some issues include solubility, bioavailability in vivo, hydrophilicity, physical and chemical instability. In addition, poor pharmacokinetics, first-pass metabolism, accumulation in tissues or low targeting efficacy can hinder therapeutic benefit. Drug-delivery systems can be employed to enhance bioavailability and pharmacological activity of molecules by enabling them to cross cellular membranes of target cells. Diseases that have benefitted from combination of drug-delivery systems with natural products include diabetes [29], cancer [30-32], neurodegenerative diseases [33] and infections [13] to name a few. Table 1 provides some examples where delivery systems have been used with selected natural compounds. In addition, Bilia has recently reviewed the role of drug-delivery systems to improve effectiveness of natural products [30].

One extensively studied natural compound encapsulated into different delivery systems to improve bioavailability via different tissues has been curcumin, a yellow pigment present in the spice turmeric (Curcuma longa) [34]. A recent review provides details of the application of delivery systems with curcumin in various diseases [35]. 
Table 1. Some examples of selected natural compounds being investigated with delivery systems.

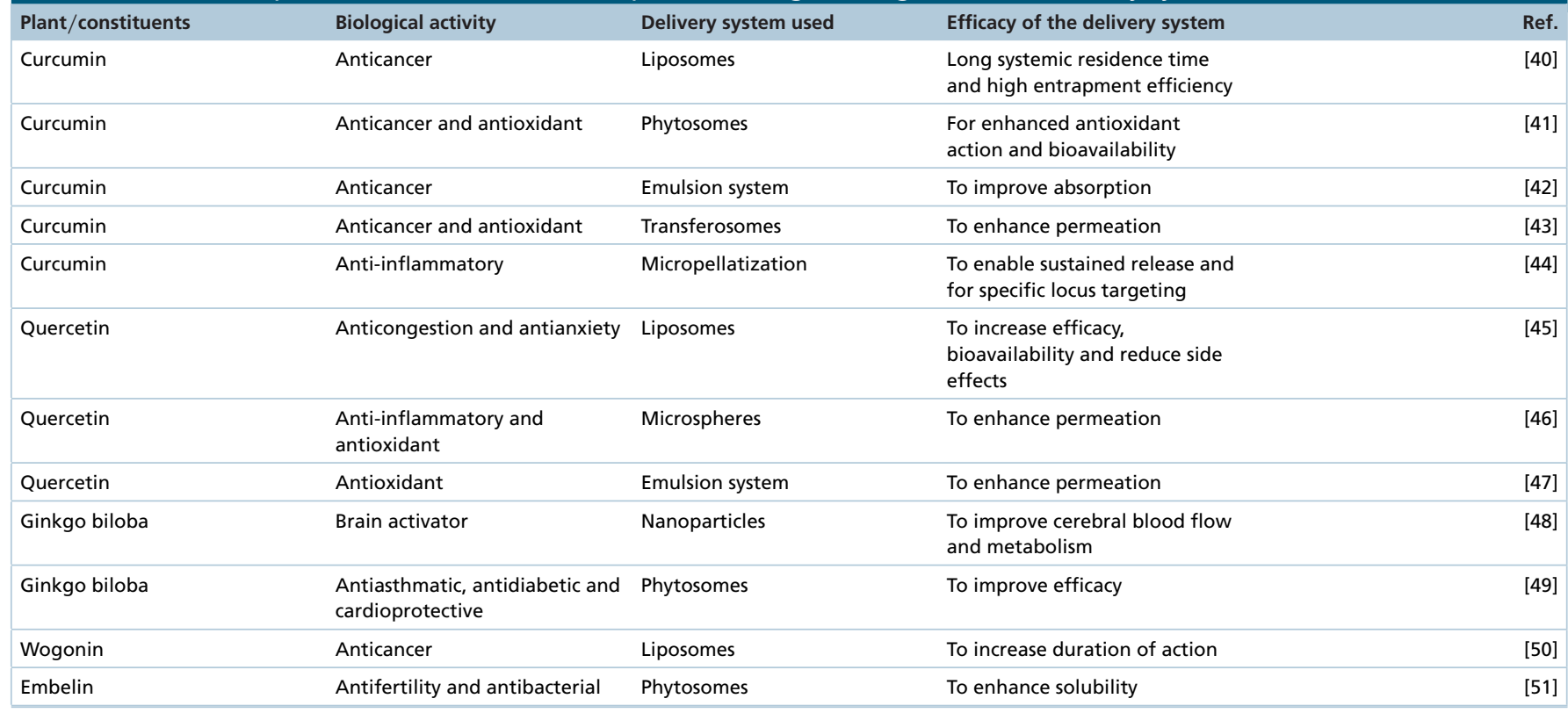

Drug-delivery systems have also been used to stabilize components and increase therapeutic activity. For example, essential oils are often unstable and susceptible to degradation, hence, encapsulation into colloidal systems is desirable [36]. Sinico et al. used multilamellar liposomes to increase therapeutic activity of Artemisia arborescens essential oil against Herpes simplex virus type 1 . They found that the oil enhanced in vitro activity by increasing the cytoplasmic viral barrier penetration of the active components of the plant [37]. Rajendran et al. used chitosan nanoparticles to encapsulate a methanolic extract of Ocimum sanctum. This formulation was demonstrated to have significant antibacterial activities against Escherichia coli, Bacillus subtilis, Pseudomonas aeruginosa and Staphylococcus aureus compared with the free unencapsulated extract [38]. One issue that can be highlighted from this area of research is the extensive use of extracts as opposed to pure compounds that makes it a problem from a regulatory perspective, as relative quantities of the active(s) may not be known. However, often several compounds present in extracts act synergistically and, so it is critical to understand synergy and adverse effects as well as the mechanisms of action when combining extracts of unknown composition and batch-to-batch variation with delivery systems [39].

Often, compounds are found to be biologically active, however, they are also highly water soluble but have low absorption (like flavonoids, tannins and terpenoids) and in some cases such as polysaccharides can have high molecular weight. This renders them unable to freely cross lipid membranes resulting in poor absorption and loss of biological activity when tested in vivo [52]. In addition, some compounds are highly toxic when exposed to normal cells [53]. In this respect, delivery systems can be employed to improve absorption, reduce toxicity and increase selectivity of some of these compounds.

Delivery systems can also provide the potential to develop therapeutics with multiple purposes. Radwan et al. described several uses of ganoderic acids (triterpenoids) extracted using methanol from the fungus Ganoderma lucidum, combined with nanoparticles for cancer therapy [54]. This particular mushroom has been in used in traditional medicine for centuries. By encapsulating a lipophilic near infrared dye within hydrophobic pockets in the polymeric matrix of polyacrylic acid-coated iron oxide nanoparticles (IONPs) together with the anticancer drug, the nanoparticles can be used for therapy as well as imaging. The development of ganoderic acid-infused nanoparticles has also enabled enhanced targeting and reduced toxic side effects by combining the anticancer drug and cancer cell-targeting moieties. The concept being that drug doses used to kill tumor cells can also affect normal cells, but use of a drug-delivery system allows accumulation of the drug in cancer cells only. In this case, folic acid was coencapsulated with gum arabic (GA) to target the folate receptor that is overexpressed in cancer cells, thus enabling higher drug doses of GA to accumulate in the tumors and reduce off-target side effects [27]. A micelle-based delivery system has also been used for enhancing the bioavailability and biodistribution of other mushroom compounds such as Flammulina velutipes sterols [54]. In these examples, delivery systems can be seen to 
have multiple purposes including their use to enhance stability, provide sustained delivery, reduce toxicity, improve targeting and offer protection from chemical or physical degradation of complex natural compounds [52,53]. The main challenge of working with mushrooms is the need to produce consistent quality of therapeutic molecules. These include difficulty in cultivating mushrooms, requirement of good manufacturing practice of cultivation methods and establishing sound methods of isolation, purification, identification and testing of bioactive compounds to elucidate the mechanisms of action [55]. The greatest problem with this area of research is the lack of standardization and this has limited the clinical trials carried out [56]. Further examples of the applications of nanoparticles for delivery of natural products have been reviewed elsewhere [52,53]. One area that might be of relevance include potent natural antibiotics, which have limited use due to insolubility issues and have required new synthesis procedures to improve their bioavailability or use of delivery systems to improve targeting and reduce the induction of antibiotic resistance $[57,58]$.

\section{Impact of drug-delivery systems in biotherapeutics developments}

The introduction of targeting moieties (such as monoclonal antibodies, peptides and proteins) into the surfaces of delivery systems can be employed to target the therapeutic agents to certain tissues, which will increase the selectivity of these plant extract formulations. This can increase the accumulation of the encapsulated product at the desired site, which will enhance its efficacy [59]. This introduces another face of the drug discovery coin that relies on the development of novel biotherapeutics. The shift in prominence toward the development of protein therapeutics (including antibodies) or nucleic acid-based drugs is in part reflected by the growing prevalence of biologic agents in the portfolio of major biopharmaceutical companies. The annual number of first approvals was in the range of 5-8 in 2014 onward, with 53 novel antibody therapeutics in Phase III studies in 2016, and approximately 210 novel antibody therapeutics in each of Phase I and II of clinical development [60]. Financially, the global sales revenue for all monoclonal antibody products was nearly US $\$ 75$ billion in 2013, and expected to reach $\$ 125$ billion by 2020. This unprecedented attraction to antibodies originates from the remarkable structural flexibility of these proteins to selectively recognize different antigen classes such as proteins, carbohydrates and lipids and challenging haptens like pharmaceutical small molecules, pesticides and even biomarkers that can contribute to the potential detection of life on other planets like Mars [61,62]. Antibodies not only represent potential therapeutics but can be implemented in diverse bespoke applications such as immunodiagnostics, biosensors, photothermal therapies and nanoparticle conjugation for drug delivery.

Several advancements have been achieved in the application of drug-delivery systems for biotherapeutics delivery. This can be desirable to enhance the onset of therapeutic action and enables administration of biomolecules via noninvasive routes such as oral or inhalation to avoid intravenous administration [63]. For example, biotherapeutics such as growth hormone, glucagon or $\alpha_{1}$-antitrypsin can be effectively delivered by inhalation therapy through the use of an appropriate delivery system [64]. Drug-delivery systems can be used to improve the pharmacokinetics of highly degradable compounds such as peptides and proteins through protecting these agents and increasing their half-lives [65]. One important development has been the delivery of insulin by noninvasive oral or inhalation routes. Insulin oral delivery is considered to be the optimum route of delivery as it will go directly to the liver, which is the main site for insulin action [66]. In addition, it will overcome the needle-anxiety barrier that is associated with delivering insulin subcutaneously [67]. However, it is highly challenging to deliver insulin using this approach for a number of reasons, including $\mathrm{pH}$ inactivation, harsh chemicals in the digestive tract, enzymatic and cellular barriers [68]. All these can lead to very low insulin bioavailability $(<1 \%)$ [69]. Recent studies have been carried out using liposomes, microspheres and microemulsions to increase the bioavailability of insulin when it is administered orally. Encapsulation of proteins like insulin in such vehicles will protect them from the harsh stomach environment [69]. In terms of inhaled insulin, the lungs have been targeted for insulin delivery due to the large surface area $\left(100 \mathrm{~m}^{2}\right)$, and the onset of action following inhalation [70]. Inhaled insulin falls into two formulations, dry powder or liquid. Dry powder formulations are considered to be more stable and require less complex devices for delivery [71]. Pfizer developed a product called Exubera (normal human insulin in dry powder formulation) that was the only approved inhaled insulin therapy to have reached the market [70,71]. It obtained approval by the US FDA in 2006. However, due to very low sales and poor patient acceptance, the product was discontinued in October 2007. A number of reasons were responsible for Exubera's failure, including the bulk and size of the inhaler device, it required weekly cleaning, patients had to perform more than one inhalation per dose to achieve the therapeutic effect [71]. Nevertheless, the potential is there when a suitable medical device is designed to overcome these problems. 
Another example of the use of drug-delivery systems with biotherapeutics is antibody-drug conjugates (ADCs). ADCs are considered a prototype of Paul Ehrlich's 'magic bullet' theory for tailored and targeted drug delivery to combat invading microbes or malignant cells. ADCs have proven their value in fatal diseases like cancer, where the standard chemotherapies are notoriously associated with limited selectivity against cancer cells leading to a small therapeutic window that can subsequently limit their efficacy [72]. The ADCs concept relies on combining the high specificity and stability profile of antibodies with the antitumor potency of very cytotoxic small-molecule drugs 'warheads' to create a selective treatment with an increased therapeutic window and reduced off-target toxicity [73]. Albeit the intensive focus of ADCs development has been on cancer, their exploitation has been extended to other applications through the optimization of immunosuppressants [74], and antibody-antibiotic conjugates [75]. The first-generation ADCs, represented by Wyeth's Mylotarg ${ }^{\circledR}$ (gemtuzumab-ozogamicin), revealed disappointing, insufficient potency and toxicity effects that led to withdrawal of this conjugate from the market in 2010. The development of more potent (100-1000 fold) cytotoxic agents, like auristatins and maytansinoid, have led to the approval of two second-generation ADCs, Acetris ${ }^{\circledR}$ (brentuximab-vedotin) and Kadcyla ${ }^{\circledR}$ (trastuzumabemtansine), by both the FDA and EMA [76,77]. Even with these approvals, the second-generation ADCs are still associated with drawbacks such as heterogeneity resulting from stochastic coupling strategies, limited penetration of solid tumors and the development of resistance [78]. Consequently, third-generation ADCs have emerged with significant focus on site-specific conjugation in order to confirm homogenous ADCs with well-defined antibodydrug ratio [79]. The high potential of these conjugates has revolutionized the biopharma R\&D, with currently over 50 ADCs undergoing clinical evaluation. According to a recent market analysis, the ADC global market is projected to display a robust growth represented by a compound annual growth rate of $22 \%$ during 2017-2022, primarily driven by a large number of $\mathrm{ADC}$ candidates in the pipeline, rising numbers of cancer patients and a wider therapeutic window offered by these ADCs [80]. Despite the wide variances among the recent conjugation approaches, which were comprehensively reviewed elsewhere [81,82], their differences have mainly focused on target selection, warhead optimisation, design of suitable linkers, selection of specific antibodies and site-specific and alternative conjugation strategies to enhance potency [72,83].

ADCs are continuously revealing great potential in both oncological and nononcological indications. A great number of drug candidates have failed in preclinical or clinical stages due to lack of selectivity or toxicity issues. For this specific reason, the ADC strategy provides a valuable opportunity for these molecules to be re-evaluated, especially with the significant advancement of antibody engineering and conjugation technologies.

Another example for the application of drug-delivery systems with biotherapeutics includes delivery of several types of nucleic acids such as plasmids, nucleotides or RNA. Drug-delivery systems enhance the application of these nucleic acid therapeutics by facilitating their accumulation and uptake by the target site. One interesting example is the use of these systems for the delivery of short-interfering RNA (siRNA). siRNA consists of short double-stranded nucleic acids that have the ability to silence specific protein expression through a mechanism called RNA interference [84]. Through this mechanism, siRNA in the cytoplasm of the target cell can cause the degradation of a specific mRNA that is complementary to the antisense strand of the siRNA, which will result in the degradation of this mRNA with the subsequent inhibition of the protein being expressed by this mRNA [85]. This results in significant research for the application of siRNA in treating different diseases such cancer. However, siRNA is a very hydrophilic molecule that cannot pass through the target cell membrane. Moreover, siRNA is highly susceptible to degradation by nuclease enzymes which limit their half-lives [86]. Drug-delivery systems such as liposomes or niosomes have been successfully applied to the delivery of siRNA in which the encapsulation of siRNA into these systems can protect them from the early degradation as well as enhance their uptake by target cells [87]. This is still an area of ongoing research, with very promising outcomes.

\section{Conclusion}

Although biotherapeutics have been developed for decades, while natural products have been around for millennia, drug-delivery systems have not been extensively utilized with them. This is particularly true for natural products, where prior to 2015 publications in this area were sparse. Since then, the advantages of combining these systems are being actively explored and are expanding the repertoire of new and safer medicines.

\section{Future perspective}

Drug discovery and drug delivery are now proceeding in parallel to improve efficacy of newly discovered drugs, increase their selectivity and bioavailability or reduce their toxicity. Delivery systems not only increase the ef- 
fectiveness of active compounds but also enable many compounds that have been discarded because of the lack of efficacy to be reinvestigated. This is an upcoming approach that has a promising future. It also provides an opportunity for pharmaceutical companies to revisit candidate molecules they have previously abandoned. This is particularly relevant to the antibiotic sector, where previously potent antibiotics have had limited use due to issues with solubility. Improved targeting with drug-delivery systems can help revitalize this area, particularly where organisms are multidrug resistant. A recent comprehensive review examines the use of nanoparticles in infection control, showing that there is renewed interest in the field [58].

\section{Financial \& competing interests disclosure}

M Obeid is funded through a PhD scholarship from Yarmouk University-Jordan; M Al-Qaraghuli is funded through an EPSRC Impact Acceleration Account (IAA)-University of Strathclyde grant (IAA grant reference number: EP/R51178X/1); A Alzahrani is funded through a PhD scholarship from the Saudi Arabian Cultural Bureau in London; K Niwasabutra is funded through a PhD scholarship from the Ministry of Science and Technology, Royal Thai Government.

The authors have no other relevant affiliations or financial involvement with any organization or entity with a financial interest in or financial conflict with the subject matter or materials discussed in the manuscript apart from those disclosed.

No writing assistance was utilized in the production of this manuscript.

\section{Executive summary}

The need for new medicines

- Increased drug resistance, for example, antibiotics, cancer chemotherapies.

- Pharmaceutical companies have moved away from drug discovery based on natural product research, however, traditional medicine is thriving in developing countries.

- New developments rising in terms of biotherapeutics.

Delivery of natural products

- Use of drug-delivery systems can improve bioavailability, biodistribution, therapeutic activity and stability of natural products.

- Introduction of multiple components into drug-delivery systems to enable a range of roles (e.g., imaging) as well as therapy.

Delivery of biotherapeutics

- Offers alternative routes of administration.

- Improved targeting of molecules.

\section{References}

Papers of special note have been highlighted as: $\bullet$ of interest

1 Peters S, Cost to develop and win marketing approval for a new drug is $\$ 2.6$ billion. (2014).

http://csdd.tufts.edu/news/complete_story/pr_tufts_csdd_2014_cost_study

2 Mack A, Relman DA, Choffnes ER. Antibiotic Resistance: Implications for Global Health and Novel Intervention Strategies: Workshop Summary. National Academies Press, Washington, D.C., USA (2011).

3 Cragg GM, Grothaus PG, Newman DJ. Impact of natural products on developing new anti-cancer agents. Chem. Rev. 109(7), 3012-3043 (2009).

4 Aqil F, Munagala R, Jeyabalan J, Vadhanam MV. Bioavailability of phytochemicals and its enhancement by drug-delivery systems. Cancer Lett. 334(1), 133-141 (2013).

5 Parveen S, Misra R, Sahoo SK. Nanoparticles: a boon to drug delivery, therapeutics, diagnostics and imaging. Nanomedicine 8(2), 147-166 (2012).

- Provides an overview on the different uses of nanoparticles not only for drug delivery, but also for imaging and diagnostics.

6 Obeid MA, Gebril AM, Tate RJ, Mullen AB, Ferro VA. Comparison of the physical characteristics of monodisperse non-ionic surfactant vesicles (NISV) prepared using different manufacturing methods. Int. J. Pharm. 521(1), 54-60 (2017).

7 Alzahrani A, Abbott G, Young L, Igoli J, Gray A, Ferro V. Phytochemical and biological investigation of Calliandra surinamensis as a potential treatment for diabetes. Planta Medica 81(S 01), P385 (2016).

8 Al Qaraghuli MM, Ferro VA. Analysis of the binding configuration and surface adaptation of different crystallized single-domain antibodies in response to various antigens. J. Mol. Recog. 30(4), (2017).

9 Gebril AM, Lamprou DA, Alsaadi MM, Stimson WH, Mullen AB, Ferro VA. Assessment of the antigen-specific antibody response induced by mucosal administration of a GnRH conjugate entrapped in lipid nanoparticles. Nanomedicine 10(5), 971-979 (2014). 
10 Miele E, Spinelli GP, Miele E et al. Nanoparticle-based delivery of small interfering RNA: challenges for cancer therapy. Int. J. Nanomed. 7, 3637-3657 (2012).

11 Hans M, Lowman A. Biodegradable nanoparticles for drug delivery and targeting. Curr. Opin. Solid State Mater. Sci. 6(4), 319-327 (2002).

12 Pelaz B, Alexiou C, Alvarez-Puebla RA et al. Diverse applications of nanomedicine. CS Nano 2017 11, 2313-2381 (2017).

13 Watkins R, Wu L, Zhang C, Davis RM, Xu B. Natural product-based nanomedicine: recent advances and issues. Int. J. Nanomed. 10, 6055 (2015).

- Provides a detailed review of improvements in natural product research.

14 Obeid MA, Khadra I, Mullen AB, Tate RJ, Ferro VA. The effects of hydration media on the characteristics of non-ionic surfactant vesicles (NISV) prepared by microfluidics. Int. J. Pharm. 516(1), 52-60 (2017).

15 Desai N. Challenges in development of nanoparticle-based therapeutics. AAPS J. 14(2), 282-295 (2012).

16 Allen TM, Cullis PR. Liposomal drug-delivery systems: from concept to clinical applications. Adv. Drug Deliv. Rev. 65(1), 36-48 (2013).

17 Zamboni WC, Torchilin V, Patri AK et al. Best practices in cancer nanotechnology: perspective from NCI nanotechnology alliance. Clin. Cancer Res. 18(12), 3229-3241 (2012).

18 Atanasov AG, Waltenberger B, Pferschy-Wenzig E-M et al. Discovery and resupply of pharmacologically active plant-derived natural products: a review. Biotechnol. Adv. 33(8), 1582-1614 (2015).

19 Shen B. A new golden age of natural products drug discovery. Cell 163(6), 1297-1300 (2015).

20 Cragg GM, Newman DJ. Natural product drug discovery in the next millennium. Pharm. Biol. 39(Suppl. 1), 8-17 (2001).

21 Newman DJ, Cragg GM. Natural products as sources of new drugs over the last 25 years. J. Nat. Prod. 70 (3), 461-477 (2007).

22 Newman DJ, Cragg GM. Natural products as sources of new drugs from 1981 to 2014. J. Nat. Prod. 79(3), 629-661 (2016).

- Comprehensive report on the trends in naturally derived drugs.

23 Bucar F, Wube A, Schmid M. Natural product isolation - how to get from biological material to pure compounds. Nat. Prod. Rep. 30(4), 525-545 (2013).

24 Sasidharan S, Chen Y, Saravanan D, Sundram K, Latha LY. Extraction, isolation and characterization of bioactive compounds from plants' extracts. Afr. J. Tradit. Complement. Altern. Med. 8(1), 1-10 (2011).

25 Niwasabutra K, Igoli J, Young L, Gray A, Ferro V. Effects of crude extracts from mushrooms on different cancer cell lines. Planta Medica 81(S 01), P320 (2016).

26 Ali S, Igoli J, Clements $\mathrm{C}$ et al. Antidiabetic and antimicrobial activities of fractions and compounds isolated from Berberis brevissima Jafri and Berberis parkeriana Schneid. Bangladesh J. Pharmacol. 8(3), 336-342 (2013).

27 Balunas MJ, Kinghorn AD. Drug discovery from medicinal plants. Life Sci. 78(5), 431-441 (2005).

28 Alonezi S, Tusiimire J, Wallace J et al. Metabolomic profiling of the effects of melittin on cisplatin resistant and cisplatin sensitive ovarian cancer cells using mass spectrometry and biolog microarray technology. Metabolites 6(4), 35 (2016).

29 Ganesan P, Arulselvan P, Choi D-K. Phytobioactive compound-based nanodelivery systems for the treatment of Type 2 diabetes mellitus-current status. Int. J. Nanomed. 12, 1097 (2017).

30 Bilia A. Improving on nature: the role of nanomedicine in the development of clinical natural drugs. Planta Medica 78(11), IL48 (2012).

31 Nam J-S, Sharma AR, Nguyen LT, Chakraborty C, Sharma G, Lee S-S. Application of bioactive quercetin in oncotherapy: from nutrition to nanomedicine. Molecules 21(1), 108 (2016).

32 Chuan L, Zhang J, Yu-Jiao Z et al. Biocompatible and biodegradable nanoparticles for enhancement of anti-cancer activities of phytochemicals. Chin. J. Nat. Med.13(9), 641-652 (2015).

33 Bagli E, Goussia A, Moschos MM, Agnantis N, Kitsos G. Natural compounds and neuroprotection: mechanisms of action and novel delivery systems. In Vivo 30(5), 535-547 (2016).

34 Prasad S, Tyagi AK, Aggarwal BB. Recent developments in delivery, bioavailability, absorption and metabolism of curcumin: the golden pigment from golden spice. Cancer Res. Treat. 46(1), 2-18 (2014).

35 Rahimi HR, Nedaeinia R, Shamloo AS, Nikdoust S, Oskuee RK. Novel delivery system for natural products: nano-curcumin formulations. Avicenna J. Phytomed. 6(4), 383 (2016).

36 Sherry M, Charcosset C, Fessi H, Greige-Gerges H. Essential oils encapsulated in liposomes: a review. J. Liposome Res. 23(4), 268-275 (2013).

37 Sinico C, De Logu A, Lai F et al. Liposomal incorporation of Artemisia arborescens L. essential oil and in vitro antiviral activity. Eur. J. Pharm. Biopharm. 59(1), 161-168 (2005).

38 Rajendran R, Radhai R, Kotresh T, Csiszar E. Development of antimicrobial cotton fabrics using herb loaded nanoparticles. Carbohydr. Polym. 91(2), 613-617 (2013).

39 Yuan H, Ma Q, Ye L, Piao G. The traditional medicine and modern medicine from natural products. Molecules 21(5), pii: E559 (2016). 
40 Li D-C, Zhong X-K, Zeng Z-P et al. Application of targeted drug delivery system in Chinese medicine. J. Control. Rel. 138(2), 103-112 (2009).

41 Bhattacharya S. Phytosomes: emerging strategy in delivery of herbal drugs and nutraceuticals. Pharma Times 41(3), 9-12 (2009).

42 Cao FH, Ouyang WQ, Wang YP et al. Study of preparation of matrin nanoemulsion and its anti-oxidation on mice. J. Northwest Sci. Tech. Univer. Agric. Forest. 3, 61-64, (2007).

43 Zhaowu Z, Xiaoli W, Yangde Z, Nianfeng L. Preparation of matrine ethosome, its percutaneous permeation in vitro and anti-inflammatory activity in vivo in rats. J. Liposome Res. 19(2), 155-162 (2009).

44 Verma H, Prasad SB, Yashwant SH. Herbal drug delivery system: a modern era prospective. Int. J. Current Pharm. Rev. Res. 4, 88-101 (2013).

$45 \mathrm{Li} \mathrm{H}, \mathrm{Li}$ S, Duan H. Preparation of liposomes containing extracts of Tripterygium wilfordii and evaluation of its stability. Zhongguo Zhong Yao Za Zhi 32(20), 2128-2131 (2007).

46 Garg R, Gupta G. Gastroretentive floating microspheres of silymarin: preparation and in vitro evaluation. Trop. J. Pharm. Res. 9(1), 58-66 (2010).

47 Pandey S, Goyani M, Devmurari V, Fakir J. Transferosomes: a novel approach for transdermal drug delivery. Der Pharmacia Lettre 1(2), 143-150 (2009).

48 Hou J, Zhou S. Formulation and preparation of glycyrrhizic acid solid lipid nanoparticles. ACTA Academiae Medicinae Militaris Tertiae 30(11), 1043-1045 (2008).

49 Sikarwar MS, Sharma S, Jain AK, Parial S. Preparation, characterization and evaluation of marsupsin-phospholipid complex. AAPS PharmSciTech 9(1), 129-137 (2008).

50 Chen Y, Lin X, Park H, Greever R. Study of artemisinin nanocapsules as anticancer drug-delivery systems. Nanomedicine 5(3), 316-322 (2009).

51 Naik SR, Panda VS. Hepatoprotective effect of Ginkgoselect Phytosome ${ }^{\circledR}$ in rifampicin induced liver injurym in rats: evidence of antioxidant activity. Fitoterapia 79(6), 439-445 (2008).

52 Bonifácio BV, Da Silva PB, Dos Santos Ramos MA, Negri KMS, Bauab TM, Chorilli M. Nanotechnology-based drug-delivery systems and herbal medicines: a review. Int. J. Nanomed. 9, 1 (2014).

- Provides insight into how drug-delivery stystems have advanced the phytochemisty field of research.

53 Saraf S. Applications of novel drug delivery system for herbal formulations. Fitoterapia 81(7), 680-689 (2010).

54 Radwan FF, Perez JM, Haque A. Apoptotic and immune restoration effects of ganoderic acids define a new prospective for complementary treatment of cancer. J. Clin. Cell Immunol. S3, 4 (2011).

55 El Enshasy HA, Hatti-Kaul R. Mushroom immunomodulators: unique molecules with unlimited applications. Trends Biotechnol. 31(12), 668-677 (2013).

56 Borchers AT, Krishnamurthy A, Keen CL, Meyers FJ, Gershwin ME. The immunobiology of mushrooms. Exp. Biol. Med. 233(3), 259-276 (2008).

57 Zaidi S, Misba L, Khan AU. Nano-therapeutics: a revolution in infection control in post antibiotic era. Nanomedicine 13(7), 2281-2301 (2017).

58 Brooks BD, Brooks AE. Therapeutic strategies to combat antibiotic resistance. Adv. Drug Deliv. Rev. 78, 14-27 (2014).

59 Grill AE, Johnston NW, Sadhukha T, Panyam J. A review of select recent patents on novel nanocarriers. Recent Pat. Drug Deliv. Formul. 3(2), 137-142 (2009).

60 Reichert J. Antibodies to watch in 2016. MAbs.9(2), 167-181 (2017).

61 Kang H-J, Kim H-J, Cha S-H. Isolation of human anti-serum albumin Fab antibodies with an extended serum-half life. Immunol. Lett. 169, 33-40 (2016).

62 Wong-Baeza C, Reséndiz-Mora A, Donis-Maturano L et al. Anti-lipid IgG antibodies are produced via germinal centers in a murine model resembling human lupus. Front. Immunol. 7, Article 396 (2016).

63 Sung JC, Pulliam BL, Edwards DA. Nanoparticles for drug delivery to the lungs. Trends Biotechnol. 25(12), 563-570 (2007).

64 Edwards DA, Ben-Jebria A, Langer R. Recent advances in pulmonary drug delivery using large, porous inhaled particles. J. Appl. Physiol. 85(2), 379-385 (1998).

65 Parveen S, Sahoo SK. Nanomedicine: clinical applications of polyethylene glycol conjugated proteins and drugs. Clin. Pharmacokinet.45(10), 965-988 (2006).

66 Ramineni SK, Cunningham LL, Dziubla TD, Puleo DA. Competing properties of mucoadhesive films designed for localized delivery of imiquimod. Biomater. Sci. 1(7), 753-762 (2013).

67 Edelman S, Pettus J. Challenges associated with insulin therapy in type 2 diabetes mellitus. Am. J. Med. 127(10), S11-S16 (2014).

68 Khafagy E-S, Morishita M, Onuki Y, Takayama K. Current challenges in non-invasive insulin delivery systems: a comparative review. Adv. Drug Deliv. Rev. 59(15), 1521-1546 (2007). 
69 Hosseininasab S, Pashaei-Asl R, Khandaghi AA et al. Synthesis, characterization, and in vitro studies of PLGA-PEG nanoparticles for oral insulin delivery. Chem. Biol. Drug Des. 84(3), 307-315 (2014).

70 Brunton S. Insulin delivery systems: reducing barriers to insulin therapy and advancing diabetes mellitus treatment. Am. J. Med. 121(6), S35-S41 (2008).

71 Cavaiola TS, Edelman S. Inhaled insulin: a breath of fresh air? A review of inhaled insulin. Clin. Ther. 36(8), 1275-1289 (2014).

72 Diamantis N, Banerji U. Antibody-drug conjugates-an emerging class of cancer treatment. Br. J. Cancer 114(4), 362-367 (2016).

73 Hughes B. Antibody-drug conjugates for cancer: poised to deliver? Nat. Rev. Drug Discov. 9(9), 665-667 (2010).

74 Talpaz M, Shah NP, Kantarjian H et al. Dasatinib in imatinib-resistant Philadelphia chromosome-positive leukemias. N. Engl. J. Med. 354(24), 2531-2541 (2006).

75 Lehar SM, Pillow T, Xu M et al. Novel antibody-antibiotic conjugate eliminates intracellular S. aureus. Nature 527(7578), 323-328 (2015).

76 Senter PD, Sievers EL. The discovery and development of brentuximab vedotin for use in relapsed Hodgkin lymphoma and systemic anaplastic large cell lymphoma. Nat. Biotechnol. 30(7), 631-637 (2012).

77 Lambert JM, Chari RV. Ado-trastuzumab Emtansine (T-DM1): an antibody-drug conjugate (ADC) for HER2-positive breast cancer. J. Med. Chem. 57, 6949-6964 (2014).

78 Vankemmelbeke M, Durrant L. Ther. Deliv. 73 141-144 Third-generation antibody drug conjugates for cancer therapy - a balancing act. (2016).

79 Kim EG, Kim KM. Strategies and advancement in antibody-drug conjugate optimization for targeted cancer therapeutics. Biomol. Ther. (Seoul) 23(6), 493-509 (2015).

80 Research and Markets Ltd R and ML. Global antibody drug conjugates (ADC) market - analysis by drugs (Adcetris, Kadcyla), pipeline drugs, regulations: opportunities and forecasts (2017-2022) [Internet]. [cited 9 May 2017]. www.researchandmarkets.com/reports/4176119/global-antibody-drug-conjugates-adc-market

81 Tolcher A. Antibody drug conjugates: lessons from 20 years of clinical experience. Ann. Oncol. 27(12), 2168-2172 (2016).

- Comprehensive overview of a biotherapeutic development that provides insight into the hurdles of translating research from the bench to the clinic.

82 Zhang Y-F, Ho M. Humanization of high-affinity antibodies targeting glypican-3 in hepatocellular carcinoma. Sci. Rep. 6, 33878 doi: 10.1038/srep33878 (2016).doi:10.1038/srep33878

83 Beck A, Goetsch L, Dumontet C, Corvaï N. Strategies and challenges for the next generation of antibody-drug conjugates. Nat. Rev. Drug Discov. 16(5), 315-337 (2017).

84 Fire A, Xu S, Montgomery MK, Kostas SA, Driver SE, Mello CC. Potent and specific genetic interference by double-stranded RNA in Caenorhabditis elegans. Nature 391(6669), 806-811 (1998).

85 Ameres SL, Martinez J, Schroeder R. Molecular basis for target RNA recognition and cleavage by human RISC. Cell 130(1), 101-112 (2007).

86 Higuchi Y, Kawakami S, Hashida M. Strategies for in vivo delivery of siRNAs. BioDrugs 24(3), 195-205 (2010).

87 Obeid MA, Elburi A, Young LC, Mullen AB, Tate RJ, Ferro VA. Formulation of non-ionic surfactant vesicles (NISV) prepared by microfluidics for therapeutic delivery of siRNA into cancer cells. Mol. Pharm. doi:10.1021/acs.molpharmaceut.7b00352 (2017) (Epub ahead of print). 


\title{
FDM 3D printing of modified drug-delivery systems using hot melt extrusion: a new approach for individualized therapy
}

\author{
Marcilio Cunha-Filho ${ }^{1}$, Maísa RP Araújo ${ }^{1}$, Guilherme M Gelfuso ${ }^{1}$ \& Tais Gratieri*,1 \\ ${ }^{1}$ Laboratory of Food, Drug \& Cosmetics (LTMAC), School of Health Sciences, University of Brasília (UnB), 70.910-900, Brasília, DF, \\ Brazil \\ *Author for correspondence: tgratieri@gmail.com
}

The production process of 3D-printed drugs offers unique advantages such as the possibility of individualizing the drug therapy and easily associating different drugs and release technologies in the same pharmaceutical unit. Fused deposition modeling, a 3D printing technique, seems especially interesting for pharmaceutical applications, due to its low cost, precise and reproducible control of the printed structures, and versatility for industrial and laboratory scale. This technique combined with another technology already adapted for the pharmaceutical industry, the hot melt extrusion, is able to incorporate various mechanisms of modified drug release. This special report aims to bring together data of the experimental progress achieved using the fused deposition modeling 3D printing combined with hot melt extrusion technique and its potential in drug delivery.

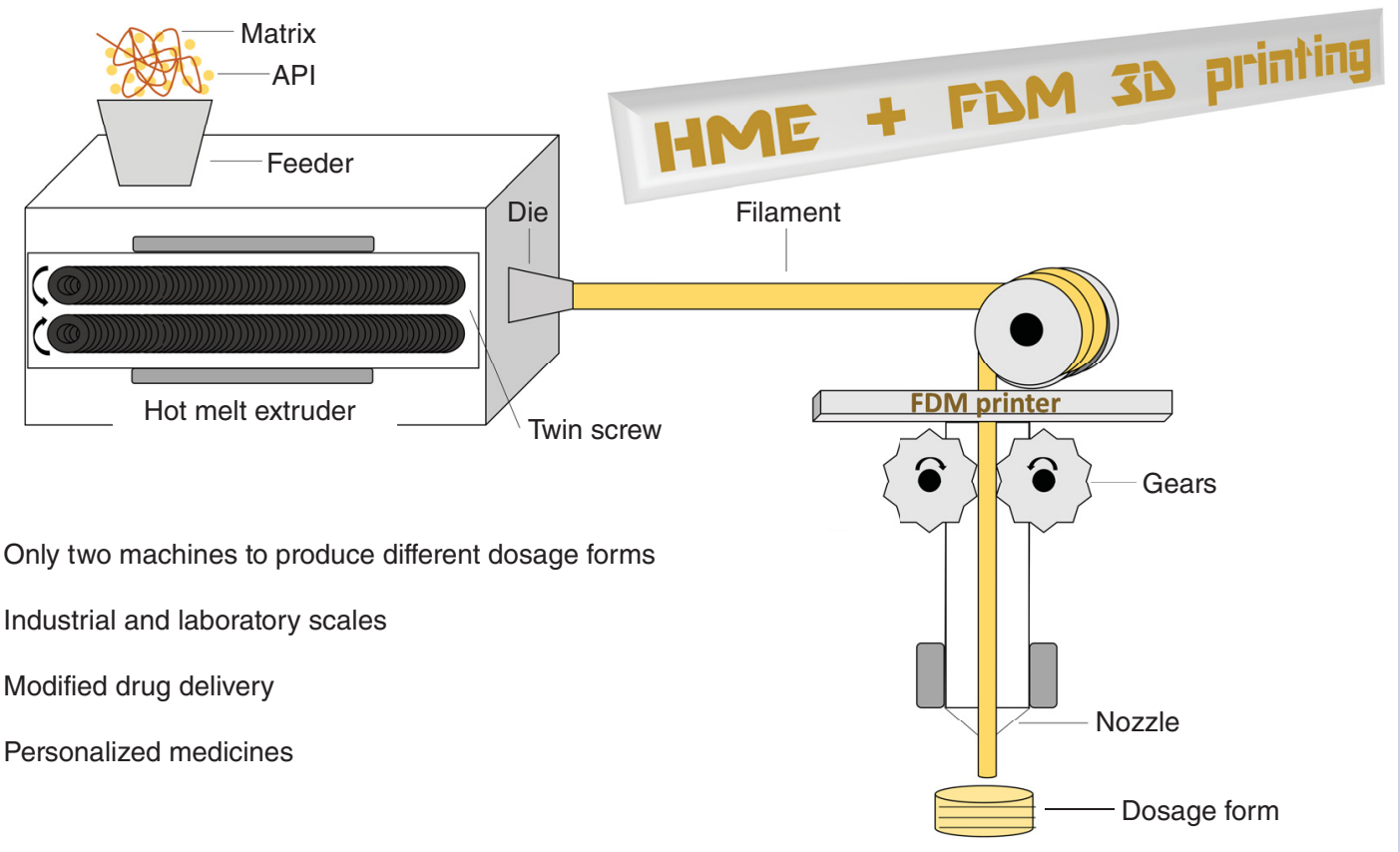

First draft submitted: 13 June 2017; Accepted for publication: 25 August 2017; Published online: 24 October 2017

Keywords: $3 \mathrm{D}$ printing $\bullet \mathrm{FDM} \bullet$ fused deposition modeling $\bullet \mathrm{HME} \bullet$ hot melt extrusion

For decades, technological advances have been launched in the pharmaceutical field as a promise of revolutionizing the segment through a new therapeutic approach involving a modified drug delivery. Emblematic examples include the Ocusert ${ }^{\circledR}$, an ophthalmic insert for glaucoma treatment from the 1970s and the osmotic pump capsule Oros ${ }^{\circledR}$ 
developed in the 1990s [1]. More recently, the so-called 'intelligent drug-delivery devices' capable of responding to patients' physiological conditions, and nanosystems developed to modulate drug release into specific organs and tissues have already become a reality [2-4].

Such new technologies have often presented insurmountable difficulties of scale-up for industrial production, mainly due to high cost and/or formulation stability issues. This explains at least in part why, despite recognized therapeutic advantages, the vast majority of currently marketed drug products do not comprise any modified drug-delivery technology. As a general rule, most pharmaceutical formulations currently marketed are nothing but a simple vehicle for storage and administration of drugs.

Still, technologies continue to evolve and 3D printing of medicines have emerged as a new feasible tool for production of modified drug-release systems, raising high hopes that it may actually be the one capable of revolutionizing the segment. Among different 3D printing technologies, for example, stereolithography, selective laser sintering, powder bed and fusion deposition modeling (FDM), FDM presents clear advantages for drugdelivery purposes. Also known as fused filament fabrication or fused filament modeling, FDM is considered of simple handling, low cost and presents the unique ability of producing dosage forms from polymeric matrices [5-7]. FDM 3D printing uses a plastic filament as an 'ink,' which is hot molded through the printer nozzle delivering consecutive layers over a platform, following desired geometry as established by a computational program [5]. There, however, resides the greatest challenge for pharmaceutical applications, such as 'plastic ink' must be biodegradable and impregnated with the drug. In this scenario, hot melt extrusion (HME) stands out among the most promising tools to produce the ideal infill materials.

HME is a technology to produce modified drug-release systems already employed in more than a dozen products marketed around the world. Besides easy processing scale-up, remarkable advantages include avoidance of organic solvents and possibility of continuous manufacturing. Also worth mentioning is HME versatility in production of modified drug-release systems (accelerated, delayed, controlled and vectorized) and the possibility of easily incorporating other technologies, for example, the insertion of nanocarriers into stable and scalable polymeric solids $[8,9]$. From recent publications, it is clear that HME potentials are broaden with the conjunction of 3D printing, which could provide higher process control capable of delivering large production of 'individualized' systems.

This review intends to analyze and discuss the challenges and future opportunities involved in coupling these two technologies.

\section{D printing of drug products}

3D printing, also known as 'additive manufacturing' and 'rapid prototyping', is the deposition of printed materials in layers in the $x, y$ and $z$-axes, following a computational control to generate a 3D structure. 3D printing is not exactly new and has already been used in a wide range of activities, as construction, automotive industry and in various areas of engineering for rapid creation of models and prototypes [10-12]. Application examples in biomedical field are recent, as production of medical implants, tissue reconstitution [13,14], and the launch of the 3D orodispersible tablet Spritam ${ }^{\circledR}$ (levetiracetam), in the USA by Aprecia Pharmaceuticals [15].

One of the greatest appeals of $3 \mathrm{D}$ printed drug products is the possibility to individualize therapy according to patient's need, which has been lost since the industrial revolution of the 19th century. Specific characteristics of each individual such as age, weight, disease stage, in addition to genetic and biological factors, demand doses and drug-release kinetics adapted to each condition [16-18]. Not coincidentally, inadequacy of drug dosage responds for $75-85 \%$ of medicine side effects [13]. The only way of adapting industrial standard pharmaceutical dosage and presentation of tablets (the most common dosage form) is through its subdivision. However, this very common practice can lead to significant dose variations also compromising drug stability, representing a serious risk to the patient [19].

$3 \mathrm{D}$ printing enables material combination, on a same printing process, to generate multilayer tablets including, not only different drugs but systems with different drug-release profiles [20,21]. Technological variables such as geometry and shape of the dosage form could be modified on demand, implying important advantages for patients with difficulty of swallowing or dysphagia [22,23].

Nonetheless, for the successful exploitation of this technology several known and still unknown challenges must be overcome. For instance, available $3 \mathrm{D}$ printers must be adapted to specific needs of drug products manufacturing, as include mechanisms for product contamination control [24]; new pharmaceutical excipients might be needed as 


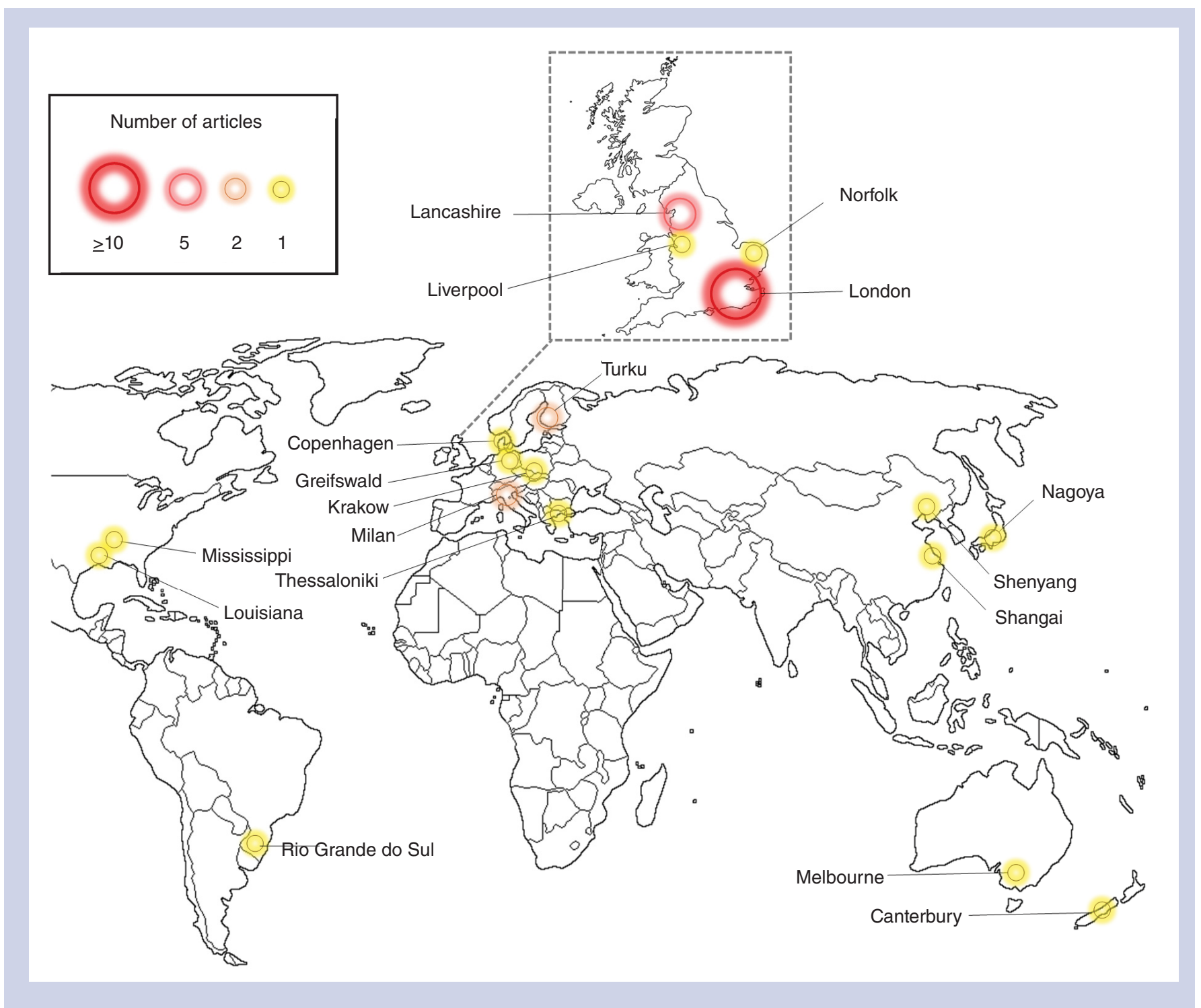

Figure 1. Geographic distribution of research paper published in fused deposition modeling 3D printing of drug-delivery systems.

well as studies to evaluate the impact of printing production variables on the quality parameters of the 3D-printed medicine.

\section{FDM 3D printing technology}

The completely automated process of 3D printing by FDM allows a surgical precision of each millimeter of the printed dosage form in terms of size, composition and shape, providing a superior reproducibility and suitability [23]. At least in theory, this technology presents less risk of generating quality deviations. Lastly, its versatility for both industrial and laboratory scales highlights the FDM process from other 3D-printing methods. 3D FDM is one of the most commercialized home printers in the world and its industrial use is well established, for example, in the production of medical devices [25]. In this sense, it is possible to glimpse a large-scale manufacturing as well as the use of FDM printer in commercial drugstores for personalized printing of the formula.

Figure 1 shows the geographic distribution of research papers published on FDM 3D printing of drugs, according to PubMed ${ }^{\circledR}$ and SciFinder ${ }^{\circledR}$ databases. More than 30 papers were published on this subject focusing mainly on tablets development and on the effect of FDM-printing parameters. Novelty is evidenced by the fact that $90 \%$ of the papers have been published in the last 30 months. Most of the work has been conducted in the UK. Start-up 
Table 1. Fused deposition modeling 3D printer parameters used in printing dosage form.

\begin{tabular}{|ll|}
\hline Parameters & Conditions tested \\
\hline Printer software & MakerWare \\
\hline Document format & Stereolithography $($. stl) \\
\hline Minimum filament length & $20 \mathrm{~cm}$ \\
\hline Filament diameter & $1.75 \pm 0.05 \mathrm{~mm}$ \\
\hline Configuration & Adjusted for PLA \\
\hline Resolution & Low $(340 \mu \mathrm{m})$, standard $(270 \mu \mathrm{m})$, high $(100 \mu \mathrm{m})$ \\
\hline Nozzle temperature & $95-250^{\circ} \mathrm{C}$ \\
\hline Plate temperature & $20-90^{\circ} \mathrm{C}$ \\
\hline Nozzle diameter & $0.20,0.25,0.30,0.40 \mathrm{~mm}$ \\
\hline Infill & $0-100 \%$ \\
\hline Layer height & $100,200,300,400,600 \mu \mathrm{m}$ \\
\hline Speed traveling & $50-150 \mathrm{~mm} / \mathrm{s}$ \\
\hline Speed extruding & $10-90 \mathrm{~mm} / \mathrm{s}$ \\
\hline Time per product & $2-5 \mathrm{~min}$ \\
\hline Raft & With and without \\
\hline Platform adherence & Scotch blue painter's tape \\
\hline PLA: Polylactic acid. & \\
\hline
\end{tabular}

companies have emerged on highlighted regions; hence, patents request involving the pharmaceutical use of 3D printing by FDM should arise in the coming months. Considering data dissemination in recent congresses, it is possible to assume many of those blanks depicted in Figure 1 will be quickly swallowed in coming months.

From commercial 3D printers commonly used for research, $78 \%$ is a MakerBot ${ }^{\circledR}$ printer model replicator 2X (NY, USA) equipped with two independent nozzles. Experiments have also been performed using Multirap M420 ${ }^{\circledR}$ (Riedhaunsen, Germany), Clouovo Delta-MK2 ${ }^{\circledR}$ (ShenYang, China), FDM-200W ${ }^{\circledR}$ (Shizuoka, Japan), Prusa i $3^{\circledR}$ (Prague, Czech Republic) and Wanhao Duplicator $4^{\circledR}$ (Zhejiang, China) $[2,3,20,24,26]$. Several parameters are required to be set up, for example, resolution, nozzle and plate temperatures, nozzle diameter, infill, layer height, speed traveling, speed extruding, extrusion rate, time per product and raft. Table 1 shows the usual machine conditions described in published papers, as determined following empirical tests [22,27,28].

Regarding 3D-printing parameters, nozzle temperature has significant importance. Filament must be heated above polymers glass transition temperature and considering the short residence time of the material in the nozzle and the small shear of the process, temperature must be higher than employed in HME. Depending on the material, temperature difference between HME and FDM could reach more than $100^{\circ} \mathrm{C}$ [29]. Molten material is then deposited in juxtaposed layers that need to stick together. The rapid cooling can affect layers bond and preclude structure construction. In this way, printing base should be thermostated, being slightly heated to allow a gradual cooling and an appropriate consolidation of the 3D structure [30].

Other important parameter is the infill of the 3D structure. Faster release of the active pharmaceutical ingredient (API) occurs for higher infill, even though the authors could not establish a relation between porosity and drugrelease rates [6]. The infill of the 3D impression can assume very small values or produce compact structures with direct reflexes on the drug release [31]. The geometric shape of 3D dosage form has also shown to influence decisively the drug release due differences in the ratio surface area and volume, as described by Goyanes et al. for tablets produced in different shapes, for example, cube, pyramid, cylinder, sphere and torus [23].

The printer resolution refers to the quality of the printed material and can be quite relevant in $3 \mathrm{D}$ printing. A high resolution leads to a more detailed printed structure, which is obtained with small movements of the extruder, and reduced the thickness of each layer, in consequence increasing the time required for printing. In fact, printing time may be the main limiting factor for this technology, since producing a regular tablet using a tablet machine takes few seconds while 3D printing can last for minutes [10]. Preliminary studies with theophylline tablets, though, have showed that resolution did not affect significantly the weight or the drug dissolution rate of 3D tablets [30]. Although this is an encouraging result for large-scale production, other studies involving different materials and more detailed analyzes are still needed. 
Some plastic polymers, such as polylactic acid (PLA), polyvinyl alcohol and acrylonitrile butadiene styrene, are ideally suited for the FDM-printing process and are very popular in the different sectors that use this technology. The pharmaceutical use of those materials, however, initially involved the drug impregnation into the commercial filaments. The simplest option for this is the immersion of the filaments into concentrated drug solutions; however, this method allows only a limited drug loading, generally below $10 \%[5,27]$. In addition, acrylonitrile butadiene styrene and PLA are insoluble in water, which produce inert matrices that present low therapeutic adherence since they remain intact throughout the entire transit of the GI tract and can provoke intestinal obstructions [32].

In such context, the previous processing of a formulation by HME may be a solution to overcome the limitations of polymers that are commonly used in 3D FDM printing, allowing the exploitation of 3D technology in the pharmaceutical field. In fact, HME can expand exponentially the materials that can be used in FDM 3D printing by obtaining filaments with higher drug loading [22].

\section{HME as an ideal match for FDM 3D printing}

HME is a technology raised in the plastic and food industries in the 1970s, which is recently being inserted in the pharmaceutical field to improve drugs' physicochemical properties and develop new drug-delivery systems [33,34]. Examples of drug products in the market containing this technology in their production are the tablets Onmel ${ }^{\mathbb{B}}$ (itraconazole), Covera-HS ${ }^{\circledR}$ (verapamil HCL) and Zithromax ${ }^{\circledR}$ (azithromycin); the intraocular implant Ozurdex ${ }^{\circledR}$ (dexamethasone); and the subcutaneous implants Zoladex ${ }^{\circledR}$ (goserelin acetate) and Implanon ${ }^{\circledR}$ (etonogestrel) [35].

The hot melt extruder is basically composed by a feeder in which the drugs and excipients are added, a barrel containing a single-screw or twin-screws where the mixing and heating of the products takes place and a die, through which the final product is ejected [35]. The high temperature and shear forces the drug to be melted and dispersed into the polymer [36]. Thus, HME can improve the bioavailability of drugs from different biopharmaceutical classes giving rise to controlled, sustained or immediately drug-release systems $[35,37]$.

The extruded filament or film produced by HME is cut in the requested size, or coupled with others devices to produce granules, pellets, transdermal implants, films, patches, modified release tablets and others [35]. Original filaments obtained by HME are themselves ideal substrates for FDM 3D printers, which can preserve, after the printing process, their modified drug-release properties. The production process from the association of these technologies consists of few steps illustrated in Figure 2.

The apparent simplicity of coupling the two technologies, however, masks a practical difficulty, which are the requirements that filaments must meet for FDM 3D printing. Concerning their dimensions, filaments need to obey narrow-range set of commercial 3D printers. Studies have demonstrated that the filament needs to be at least $20-\mathrm{cm}$ longer in order to go through the printer's feed gears [38]. Additionally, round shape and regular diameter are needed according to printer nozzle. For the most commonly used nozzle $(0.20-\mathrm{mm}$ diameter), filament must have a diameter of $1.75 \pm 0.05 \mathrm{~mm}[29,30]$. Other ideal mechanical and thermoplastic requirements are not completely clear yet. For example, filaments must be resistant and flexible at the same time, so they do not break into the printer [29,39]. Nonetheless, viscoelastic properties can obviously be modified by other components addition [29]. The class of materials most recommended to change polymers properties is plasticizers, which are low-molecular-weight compounds that increase free space between the polymer chains and improve its processing conditions [22,40]. More specifically, plasticizers can flexibilize the polymer and reduce the polymer glass transition temperature, which should enable the use of thermolabile drugs [11]. The popular HME pharmaceutical polymers, Soluplus ${ }^{\circledR}$ and Eudragit $\mathrm{E}^{\circledR}$, have not shown appropriate filament for FDM 3D printing. In such case, the use of polymer blends or its association with fillers (Table 2) could overcome these issues, interfering directly in filament rheological properties [22,41]. Oily lubricants with high melting points, like castor oil and oleic acid, were used around the filaments during the $3 \mathrm{D}$ printing, to avoid sticking of the filament inside the printer and clogging of the nozzle. According to some published studies, use of these components does not influence the drug stability or its dissolution rate $[27,28,42]$.

Polymers usually exploited in FDM 3D printing as well as several polymers used in HME not yet evaluated are listed in Table 2. Other pharmaceutical adjuvants and drugs tested so far using FDM 3D printing are also included. Noticeably, the number of drugs tested using this technology is still quite low. Nonetheless, stability maintenance after thermal processing by both $\mathrm{HME}$ and $3 \mathrm{D}$ printing was achieved in all the drugs tested, in other words, theophylline, captopril and prednisolone, even following manipulation at temperatures above $100^{\circ} \mathrm{C}$ [22].

The range of polymers evaluated to produce release systems by HME/FDM 3D printing includes well-known materials such as PLA, polyglycolic acid, polyvinylpyrrolidone, Eudragits ${ }^{\circledR}$, cellulose derivatives, ethylene vinyl 

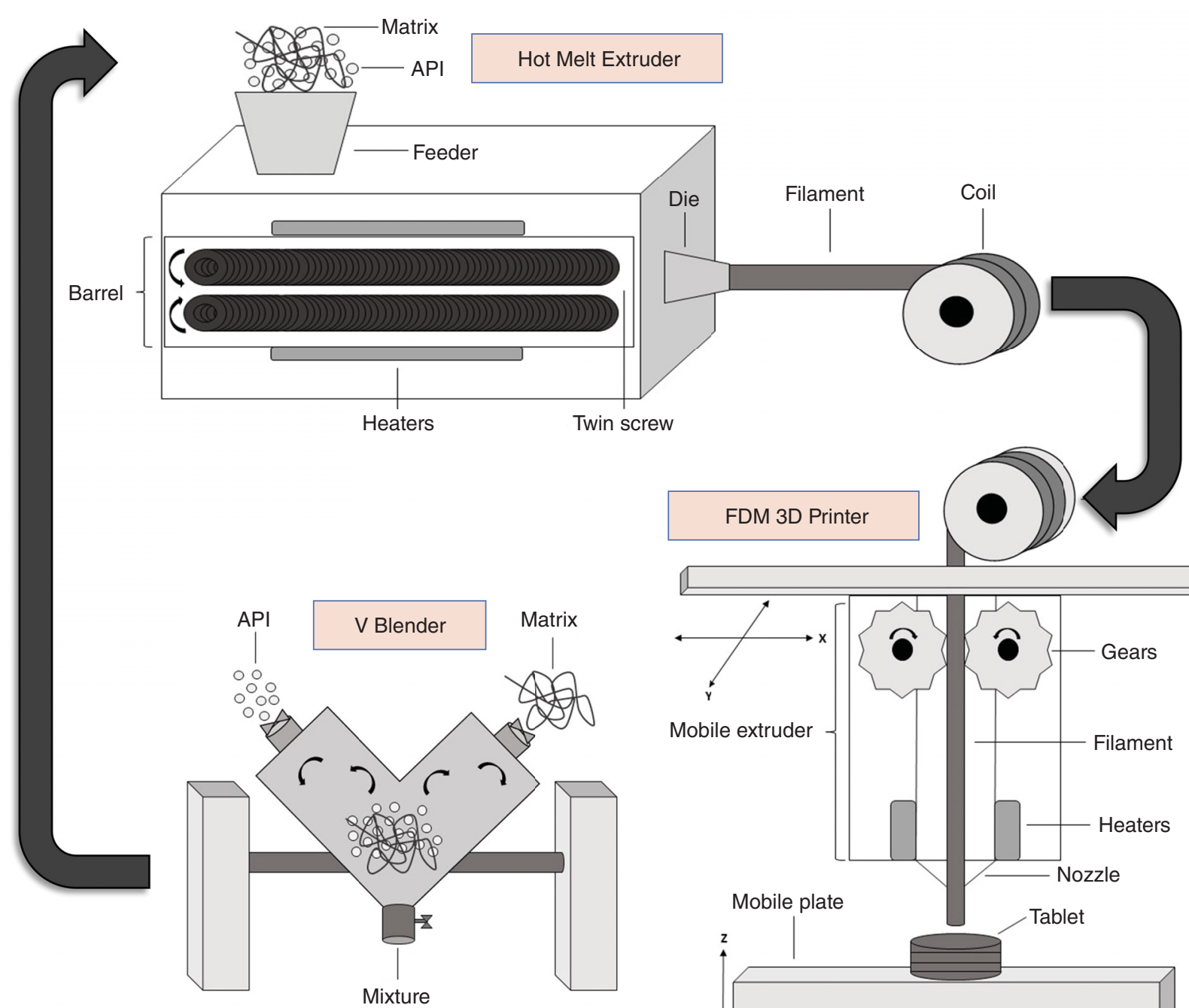

DM 3D Printer
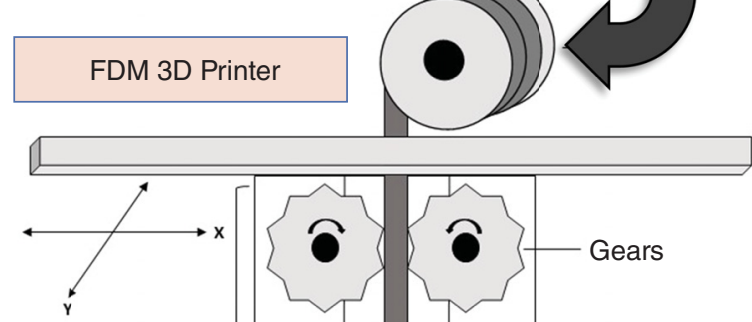

Mobile extruder

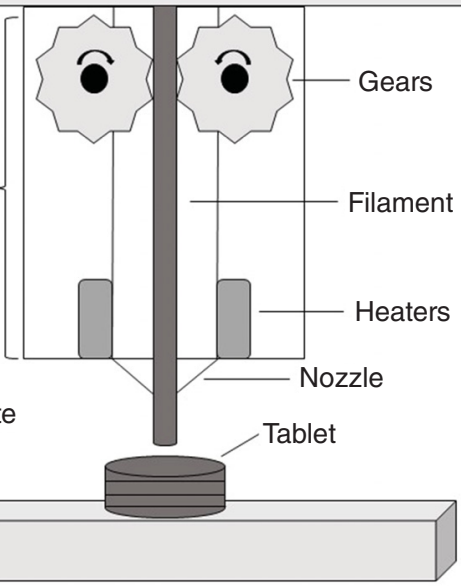

Figure 2. Schematic of the producing process of fused deposition modeling 3D printing of drug products using hot melt extrusion filaments.

acetate and hypromellose acetate succinate $[22,29,30,39,43]$. Still, several others as chitosan, xanthan gum, polyacrylic acid, polyglycolic acid, already used in HME systems could be exploited (Table 2) [35].

\section{Drug-delivery systems using FDM 3D printing \& the potential of 3D nanocarriers}

Researchers have preferably focused on the production of oral tablets with modified drug delivery with HME/FDM $3 \mathrm{D}$-printing association, since the most diverse release profiles can be yield, for example, immediate release [22,28,30], delayed release [42], pulsatile release [38], enteric release [44] and controlled release [14,30,31]. The possibility of combining different drug-release profiles has already been probed in the development of double-drug tablets containing acetaminophen and caffeine, in which the drug release was fast for the coating tablet layer and retarded for the matrix content [21]. Also, topical drug delivery has been successfully explored through $3 \mathrm{D}$ printing of a nose mask containing an antiacne drug and antimicrobial wound dressings personalized according to the anatomy of the patient using a $3 \mathrm{D}$ scanning $[45,46]$. The potential of 3D technology application on pharmaceutical field, however, might be yet to be revealed. Wider applications might appear with the $3 \mathrm{D}$ printing of nanosystems. In fact, the first experimental approach coupling nanotechnology and 3D FDM has just been published. 3D tablets elaborated 
Table 2. Components utilized to produce drug-delivery systems by fused deposition modeling 3D printing using hot melt extrusion filaments and pharmaceutical materials with potential for this use.

Component
Raw material

Polylactic acid (PLA), poly- $\varepsilon$-caprolactone (PCL), polyurethane (PU), polyvinyl alcohol (PVA), polyvinylpyrrolidone (PVP), Eudragit, polyvinyl caprolactam-polyvinyl acetate-polyethylene glycol graft (SLP), polyvinyl alcohol-polyethylene glycol graft (KIR), hydroxypropyl cellulose (HPC), hydroxypropyl methyl cellulose (HPMC), ethyl cellulose (EC), hydroxypropyl methyl cellulose acetate succinate (HPMCAS), poly-L-lactide (PLLA), ethylene vinyl acetate (EVA)

Pharmaceutical HME polymers not yet used in 3D FDM

Polyacrylic acid (PAA), polyglycolic acid (PGA), polylactide-co-glycolide, $\varepsilon$-caprolactone, L,D-lactide, glycolide, polyortho esters (POE I, II, III, IV), polyanhydride, starch, chitosan and xanthan gum

Fillers and plasticizers

Triethyl citrate (TEC), tricalcium phosphate (TCP), tribasic phosphate (TBP), triacetin, talc, spray-dried lactose (SD), directly compressible lactose (DC), microcrystalline cellulose (MMC), polyethylene glycol (PEG), polysorbate, polyethylene oxide (PEO), methylparaben, mannitol

Lubricants

Active pharmaceutical ingredients

Theophylline, 5-ASA, 4-ASA, captopril, prednisolone, budesonide, paracetamol, caffeine, salicylic acid, dipyridamole, felodipine, acetaminophen, furosemide, fluorescein sodium salt, quinine, indomethacin, nitrofurantoin monohydrate, nitrofurantoin anhydrate, hydroxyapatite, glipizide, curcumin, disulfiram, zinc, copper, silver, gentamicin, methotrexate, deflazacort

FDM: Fused deposition modeling; HME: Hot melt extrusion.

with Eudragit and poly- $\varepsilon$-caprolactone were impregnated with deflozacort nanocapsules, converting nanocapsule suspensions into a solid dosage forms [47].

In general, nanosystems' production generates toxic organic solvent residues and does not allow a continuous fabrication process. Those methods also produce particles that can be uneven and instable, leading to aggregation. HME in some circumstances can also circumvent those problems [48,50]. Nanocrystal solid dispersions of efavirez were obtained with high-pressure homogenization associated with HME, resulting in a highly stable formulation after a real-time-used storage conditions [48]. Solid-lipid nanoparticles of fenofibrate were produced using HME in a continuous and large-scale process, revealing important improvement in drug pharmacokinetic parameters [51]. HME has also been employed to convert a liquid-stabilized nanosuspension into a solid formulation in a one-step process by removing aqueous solvent following devolatilization, which successfully resulted in solidified polymer at the outlet-forming filaments containing nanocrystals [4]. Despite the limited number of studies, HME technology has demonstrated great feasibility as a vehicle for nano-based drug-delivery systems. Nevertheless, many advances need to be made, especially in order to achieve a better insertion of the nanosystems elaboration directly into the HME process.

\section{Conclusion}

The results obtained so far in the development of drug products using HME associated with FDM 3D reinforce the envisioned potential of this technology of being capable of bringing modified drug-delivery systems to people's daily lives. Nevertheless, the adaptation of FDM 3D printing for pharmaceutical manufacturing needs to be better understood, especially regarding printer adjustments and expansion of available pharmaceutical materials.

\section{Future perspective}

When a retrospective of the main innovations in the pharmaceutics field is taken in account, it is possible to affirm that no other technology attracted so much attention and created so many expectations as the $3 \mathrm{D}$ printing of drug products. However, the greatest challenge for FDM 3D-printing technique application is to obtain a suitable filament with appropriate drug loading. HME appears in this scenario as a perfect match for FDM 3D technology, being capable of producing filaments with high drug-load capacity that can modulate delivery of drugs. Hence, in the next few years, it is possible to foresee, not only these, but novel materials being tested for pharmaceutical purposes. Stability concern may remains for all heat-based or products that need thermal treatments. Nevertheless, with technology evolution novel machinery design with actual coupling of thermal extruders and 3D printers may overcome difficulties in meeting filament requirements and probably allow for a proper quality control, as nowadays 
critical aspects to meet product quality remain unclear. In a few years, after meeting primary industrial production and regulation challenges, personalized medicines may become a reality.

\section{Financial \& competing interests disclosure}

The authors' research has been founded by grants from the National Council for the Research and Development (CNPq) of Brazilian Government, Coordination for Higher Education Personnel Improvement (CAPES) and Research support foundation of the Federal District (FAPDF). MRP Araújo's scholarship is provided by CAPES. The authors have no other relevant affiliations or financial involvement with any organization or entity with a financial interest in or financial conflict with the subject matter or materials discussed in the manuscript apart from those disclosed.

No writing assistance was utilized in the production of this manuscript.

\section{Executive summary}

\section{D printing of drugs}

- Individual characteristics such as age, weight, stage of disease, in addition to genetic and biological factors, demand doses and drug-release kinetics adapted to each condition.

- 3D printing can provide the association of different drugs, high control of the printing process, modification in the geometry and dosage of the pharmaceutical form, adapting it to each patient needs.

- Drug-delivery devices (immediate, prolonged, controlled and vectorized) or therapeutic nanosystems can be produced affordably and in large scale.

\section{Fused deposition modeling 3D printing of drugs}

- Fused deposition modeling (FDM) is more suitable for drug production because of its simple handling, low cost and the use of polymeric matrices, although high temperature can degrade thermosensitive drugs.

- There are critical parameters in the printer that need to be observed, such as resolution, temperature, nozzle diameter, infill, layer height, speed and raft.

- There are polymers, fillers, plasticizers and active pharmaceutical ingredients already studied to produce filaments.

Hot melt extrusion filaments for FDM 3D printing of drugs

- FDM printing combined with the hot melt extrusion (HME) technique can improve the solubility of poorly soluble drugs and assists in more uniform dispersion of the drug in the polymeric medium.

- To produce HME filaments, the active pharmaceutical ingredient and the excipients are inserted on the HME feeder for heating and mixing, preferably by twin-screw, and the homogenized product is collected as a filament.

\section{References}

1 Hoffman AS. The origins and evolution of 'controlled' drug-delivery systems. J. Control. Rel. 132(3), 153-163 (2008).

2 Kempin W, Franz C, Koster LC et al. Assessment of different polymers and drug loads for fused deposition modeling of drug loaded implants. Eur. J. Pharm. Biopharm. 115, 84-93, (2017).

3 Zhang J, Feng X, Patil H, Tiwari RV, Repka MA. Coupling 3D printing with hot-melt extrusion to produce controlled-release tablets. Int. J. Pharm. 519(1-2), 186-197 (2017).

4 Khinast J, Baumgartner R, Roblegg E. Nano-extrusion: a one-step process for manufacturing of solid nanoparticle formulations directly from the liquid phase. AAPS. PharmSciTech. 14(2), 601-604 (2013).

5 Goyanes A, Buanz AB, Basit AW, Gaisford S. Fused-filament 3D printing (3DP) for fabrication of tablets. Int. J. Pharm. 476(1-2), 88-92 (2014).

6 Goyanes A, Kobayashi M, Martínez-Pacheco R, Gaisford S, Basit AW. Fused-filament 3D printing of drug products: microstructure analysis and drug release characteristics of PVA-based caplets. Int. J. Pharm. 514(1), 290-295 (2016).

7 Wang J, Goyanes A, Gaisford S, Basit AW. Stereolithographic (SLA) 3D printing of oral modified-release dosage forms. Int. J. Pharm. 503(1-2), 207-212 (2016).

8 Patil H, Tiwari RV, Repka MA. Hot-melt extrusion: from theory to application in pharmaceutical formulation. AAPS. PharmSciTech. 17(1), 20-42 (2016).

9 Tiwari RV, Patil H, Repka MA. Contribution of hot-melt extrusion technology to advance drug delivery in the 21 st century. Expert. Opin. Drug. Deliv. 13(3), 451-464 (2016).

10 Norman J, Madurawe RD, Moore CM, Khan MA, Khairuzzaman A. A new chapter in pharmaceutical manufacturing: 3D-printed drug products. Adv. Drug. Deliv. Rev. 108, 39-50 (2017).

11 Prasad LK, Smyth H. 3D Printing technologies for drug delivery: a review. Drug. Dev. Ind. Pharm. 42(7), 1019-1031 (2016). 
12 Yu DG, Zhu LM, Branford-White CJ, Yang XL. Three-dimensional printing in pharmaceutics: promises and problems. J. Pharm. Sci. 97(9), 3666-3690 (2008).

13 Alhnan MA, Okwuosa TC, Sadia M, Wan KW, Ahmed W, Arafat B. Emergence of 3D printed dosage forms: opportunities and challenges. Pharm. Res. 33(8), 1817-1832 (2016).

14 Masood SH. Application of fused deposition modelling in controlled drug delivery devices. Assembly Automation 27(3), 215-221 (2007).

15 Aprecia Pharmaceuticals. FDA approves the first 3D printed drug product. (2015). www.aprecia.com/pdf/ApreciaSPRITAMLaunchPressRelease__FINAL.PDF

16 Planchette C, Pichler H, Wimmer-Teubenbacher M. Printing medicines as orodispersible dosage forms: effect of substrate on the printed micro-structure. Int. J. Pharm. 509(1-2), 518-527 (2016).

17 Raijada D, Genina N, Fors D et al. A step toward development of printable dosage forms for poorly soluble drugs. J. Pharm. Sci. 102(10), 3694-3704 (2013).

18 Kolakovic R, Viitala T, Ihalainen P, Genina N, Peltonen J, Sandler N. Printing technologies in fabrication of drug-delivery systems. Expert. Opin. Drug. Deliv. 10(12), 1711-1723 (2013).

19 Teixeira MT, Sá-Barreto LCL, Gratieri T, Gelfuso GM1, Silva ICR, Cunha-Filho MSS. Key technical aspects influencing the accuracy of tablet subdivision. AAPS. PharmSciTech. 18(4), 1393-1401 (2017).

20 Li Q, Wen H, Jia D et al. Preparation and investigation of controlled-release glipizide novel oral device with three-dimensional printing. Int. J. Pharm. 525(1), 5-11 (2017).

21 Goyanes A, Wang J, Buanz A et al. 3D printing of medicines: engineering novel oral devices with unique design and drug release characteristics. Mol. Pharm. 12(11), 4077-4084 (2015).

22 Sadia M, Sośnicka A, Arafat B et al. Adaptation of pharmaceutical excipients to FDM 3D printing for the fabrication of patient-tailored immediate release tablets. Int. J. Pharm. 513(1-2), 659-668 (2016).

23 Goyanes A, Robles Martinez P, Buanz A, Basit AW, Gaisford S. Effect of geometry on drug release from 3D printed tablets. Int. J. Pharm. 494(2), 657-663 (2015).

24 Davies MJ, Costley E, Ren J, Gibbons P, Kondor A, Naderi M. On drug-base incompatibilities during extrudate manufacture and fused deposition 3D printing. J. 3D Print. Med. 1(1), 31-47 (2017).

25 Cicala G, Latteri A, Del Curto B, Lo Russo A, Recca G, Farè S. Engineering thermoplastics for additive manufacturing: a critical perspective with experimental evidence to support functional applications. J. Appl. Biomater. Funct. Mater. 15(1), 0 (2017).

26 Tagami T, Fukushige K, Ogawa E, Hayashi N, Ozeki T. 3D printing factors important for the fabrication of polyvinylalcohol filament-based tablets. Biol. Pharm. Bull. 40(3), 357-364 (2017).

27 Skowyra J, Pietrzak K, Alhnan MA. Fabrication of extended-release patient-tailored prednisolone tablets via fused deposition modelling (FDM) 3D printing. Eur. J. Pharm. Sci. 68, 11-17 (2015).

28 Okwuosa TC, Stefaniak D, Arafat B, Isreb A, Wan KW, Alhnan MA. A lower temperature FDM 3D printing for the manufacture of patient-specific immediate release tablets. Pharm. Res. 33(11), 2704-2712 (2016).

29 Melocchi A, Parietti F, Maroni A, Foppoli A, Gazzaniga A, Zema L. Hot-melt extruded filaments based on pharmaceutical grade polymers for 3D printing by fused deposition modeling. Int. J. Pharm. 509(1-2), 255-263 (2016).

30 Pietrzak K, Isreb A, Alhnan MA. A flexible-dose dispenser for immediate and extended release 3D printed tablets. Eur. J. Pharm. Biopharm. 96, 380-387 (2015).

31 Goyanes A, Buanz AB, Hatton GB, Gaisford S, Basit AW. 3D printing of modified-release aminosalicylate (4-ASA and 5-ASA) tablets. Eur. J. Pharm. Biopharm. 89, 157-162 (2015).

32 Long J, Gholizadeh H, Lu J, Bunt C, Seyfoddin A. Application of fused deposition modelling (FDM) method of 3D printing in drug delivery. Curr. Pharm. Des. 23(3), 433-439 (2017).

33 Maniruzzaman M, Rana MM, Boateng JS, Mitchell JC, Douroumis D. Dissolution enhancement of poorly water-soluble APIs processed by hot-melt extrusion using hydrophilic copolymers. Drug. Dev. Ind. Pharm. 39(2), 218-227 (2013).

34 Maniruzzaman M, Boateng JS, Bonnefille M, Aranyos A, Mitchell JC, Douroumis D. Taste masking of paracetamol by hot-melt extrusion: an in vitro and in vivo evaluation. Eur. J. Pharm. Biopharm. 80(2), 433-442 (2012).

35 Stanković M, Frijlink HW, Hinrichs WL. Polymeric formulations for drug release prepared by hot melt extrusion: application and characterization. Drug. Discov. Today. 20(7), 812-823 (2015).

Zhang Y, Luo R, Chen Y, Ke X, Hu D, Han M. Application of carrier and plasticizer to improve the dissolution and bioavailability of poorly water-soluble baicalein by hot melt extrusion. AAPS. PharmSciTech. 15(3), 560-568 (2014). Moulton SE, Wallace GG. 3-dimensional (3D) fabricated polymer based drug-delivery systems. J. Control. Rel. 193, 27-34 (2014). 
39 Genina N, Holländer J, Jukarainen H, Mäkilä E, Salonen J, Sandler N. Ethylene vinyl acetate (EVA) as a new drug carrier for 3D printed medical drug delivery devices. Eur. J. Pharm. Sci. 90, 53-63 (2016).

40 Madan S, Madan S. Hot melt extrusion and its pharmaceutical applications. Asian. J. Pharm. Sci. 7(2), 123-133 (2012).

41 Alhijjaj M, Belton P, Qi S. An investigation into the use of polymer blends to improve the printability of and regulate drug release from pharmaceutical solid dispersions prepared via fused deposition modeling (FDM) 3D printing. Eur. J. Pharm. Biopharm. 108, 111-125 (2016).

42 Okwuosa TC, Pereira BC, Arafat B, Cieszynska M, Isreb A, Alhnan MA. Fabricating a shell-core delayed release tablet using dual FDM 3D printing for patient-centred Therapy. Pharm. Res. 34(2), 427-437 (2017).

43 Goyanes A, Fina F, Martorana A, Sedough D, Gaisford S, Basit AW. Development of modified release 3D printed tablets (printlets) with pharmaceutical excipients using additive manufacturing. Int. J. Pharm. 527(1-2), 21-30 (2017).

44 Goyanes A, Chang H, Sedough D et al. Fabrication of controlled-release budesonide tablets via desktop (FDM) 3D printing. Int. J. Pharm. 496(2), 414-420 (2015).

45 Goyanes A, Det-Amornrat U, Wang J, Basit AW, Gaisford S. 3D scanning and 3D printing as innovative technologies for fabricating personalized topical drug-delivery systems. J. Control. Rel. 234, 41-48 (2016).

46 Hassan ZM, Goyanes A, Clark V, Basit AW, Hilton ST, Gaisford S. Patient-specific 3D scanned and 3D printed antimicrobial polycaprolactone wound dressings. Int. J. Pharm. 0378-5173(17), 30387-30393 (2017).

47 Beck RCR, Chaves PS, Goyanez A et al. 3D printed tablets loaded with polymeric nanocapsules: an innovative approach to produce customized drug-delivery systems. Int. J. Pharm. 0378-5173(17), 30498-30502 (2017).

48 Ye X, Patil H, Feng X et al. Conjugation of hot-melt extrusion with high-pressure homogenization: a novel method of continuously preparing nanocrystal solid dispersions. AAPS. PharmSciTech. 17(1), 78-88 (2016).

49 Waard H, Frijlink HW, Hinrichs WL. Bottom-up preparation techniques for nanocrystals of lipophilic drugs. Pharm. Res. 28(5), 1220-1223 (2011).

50 Repka MA, Majumdar S, Kumar Battu S, Srirangam R, Upadhye SB. Applications of hot-melt extrusion for drug delivery. Expert. Opin. Drug. Deliv. 5(12), 1357-1376 (2008).

51 Patil H, Feng X, Ye X, Majumdar S, Repka MA. Continuous production of fenofibrate solid lipid nanoparticles by hot-melt extrusion technology: a systematic study based on a quality by design approach. AAPS. J. 17(1), 194-205 (2015). 


\title{
Therapeutic Delivery
}

\section{Potential enhancement and targeting strategies of polymeric and lipid-based nanocarriers in dermal drug delivery}

\author{
Emine Kahraman ${ }^{1}$, Sevgi Güngör ${ }^{1}$ \& Yıldız Özsoy ${ }^{*}, 1$ \\ ${ }^{1}$ Department of Pharmaceutical Technology, Faculty of Pharmacy, Istanbul University, 34116, Istanbul, Turkey \\ * Author for correspondence: Tel: +90 21244002 72; Fax: +90 21244002 52; yozsoy@istanbul.edu.tr
}

\begin{abstract}
Nanocarriers used for alternative drug-delivery strategies have gained interest due to improved penetration and delivery of drugs into specific regions of the skin in recent years. Dermal drug delivery via polymeric-based nanocarriers (polymeric nanoparticles, micelles, dendrimers) and lipid-based nanocarriers (solid-lipid nanoparticles and nanostructured lipid carriers, vesicular nanocarriers including liposomes, niosomes, transfersomes and ethosomes) has been widely investigated. Although penetration of nanocarriers through the intact skin could be restricted, these carriers are particularly considered as feasible for the treatment of dermatological diseases in which the skin barrier is disrupted and also for follicular delivery of drugs for management of skin disorders such as acne. This review mainly highlights the recent approaches on potential penetration enhancement and targeting mechanisms of these nanocarriers.
\end{abstract}

First draft submitted: 11 July 2017; Accepted for publication: 1 September 2017; Published online: 24 October 2017

Keywords: dermal drug delivery • enhanced skin penetration • follicular targeting • lipid nanocarriers $\bullet$ polymeric nanocarriers

The dermal delivery has proposed transport of drugs into targeted areas in the skin. But, the skin is an excellent barrier preventing the penetration of drugs into its deeper layers due to the defensive membrane structure of the stratum corneum, which is the uppermost layer of the skin. A lot of penetration enhancement strategies have been utilized to overcome this barrier [1].

In the last two decades, nanosized drug carriers have been widely explored to improve skin penetration of drugs and examined dermal targeting strategy in the treatment of dermatological diseases. The nanocarriers have also superiorities such as improving chemical drug stability and limiting side effects of drugs by providing drug release behavior over prolonged periods of time [2,3]. Although skin penetration of nanocarriers through the intact skin could be restricted, these carriers could provide advantages for the treatment of diseases including atopic dermatitis, and psoriasis in which skin barrier is disrupted and requiring follicular delivery such as acne.

The potential applications of various nanocarriers in dermal drug delivery have intensively been handled out in excellent review articles [4-6] and reviewed in book chapters [7,8] until now. The aim of the present review is to give an overview to enhance transport and targeting of drugs into desired skin areas via polymeric-based nanocarriers (polymeric nanoparticles, micelles and dendrimers) and lipid-based nanocarriers (solid-lipid nanoparticles [SLNs] and nanostructured lipid carriers [NLCs], vesicular nanocarriers including liposomes, niosomes, transfersomes and ethosomes). This review article explains the potential penetration enhancement mechanisms and targeting strategies for diverse types of nanocarriers in a detailed and systematic manner, which proposes a different view to the other review articles related to nanocarriers.

\section{The skin structure \& penetration pathways}

The human skin is a unique membrane due to the well-designed structure. It represents a rigid physical barrier that protects the organism from external environmental factors and, regulates transepidermal water loss from the body. The skin comprises three main layers called as epidermis, dermis and hypodermis (subcutaneous tissue) [9] (Figure 1). The epidermis is divided into two main layers: the stratum corneum and viable epidermis. The stratum 

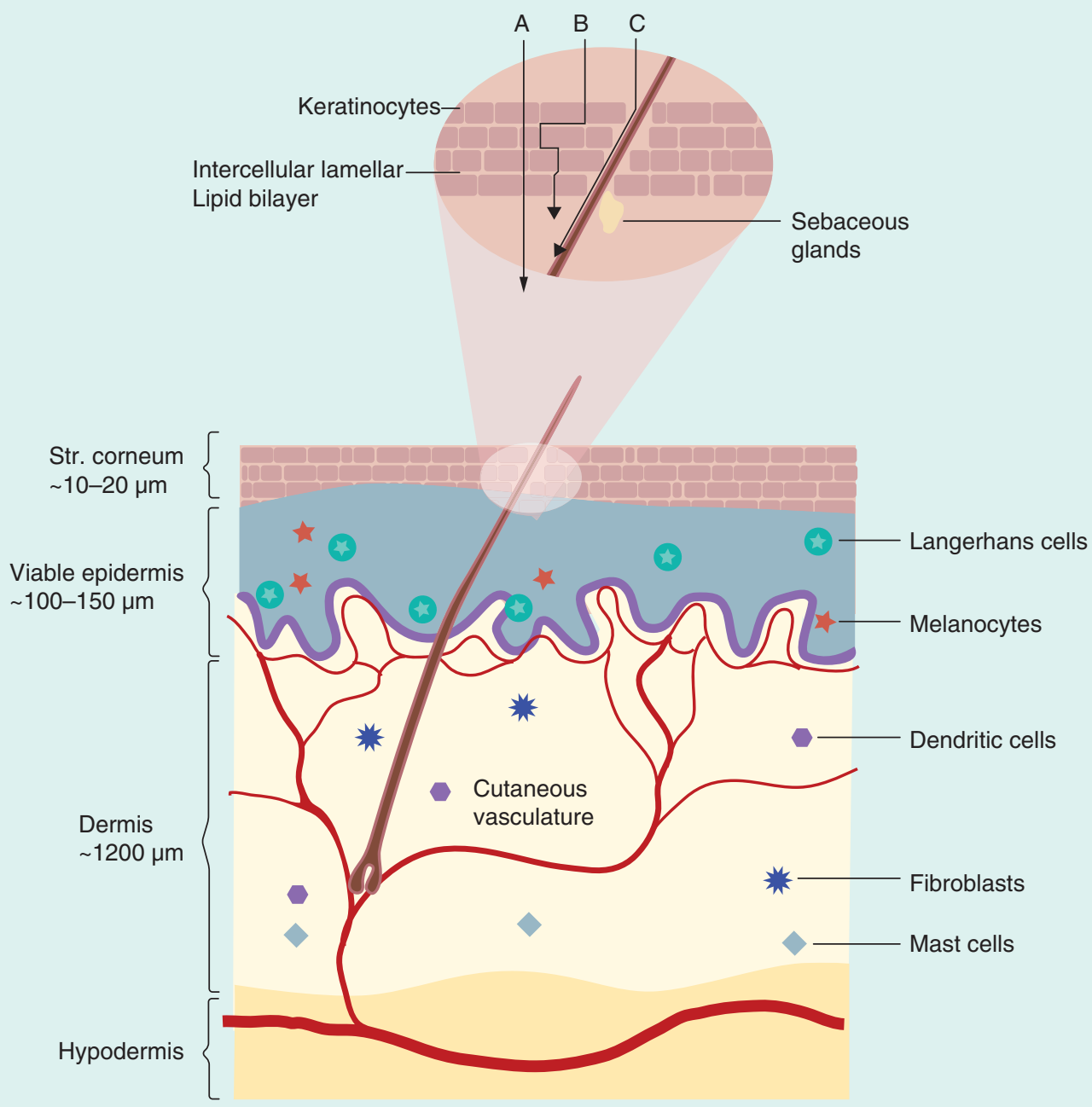

Figure 1. The skin structure and penetration pathways. (A) Transcellular, (B) intercellular and (C) transappendageal routes.

corneum, the uppermost layer of the epidermis, generates major defensive functions of the skin. It is composed of dead, flattened, keratin-rich cells (keratinocytes) and intercellular lamellar lipid bilayers. Although its thickness is only $10-20 \mu \mathrm{m}$, the stratum corneum is considered as a rate-limiting barrier for diffusion of drugs. Topically applied compounds should be delivered across that barrier to penetrate either into skin strata or through the skin. The viable epidermis ( 100-150 $\mu \mathrm{m}$ thickness) comprises keratinocytes differentiated by corneocytes, Langerhans cells (antigen-presenting immune cells) and melanocytes that produce the protective skin-darkening pigment melanin.

The dermis comprises fibrous proteins such as collagen, elastin and fibronectin and glycosaminoglycan matrix in which there are hyaluronic acid (HA) and chondroitin sulfates. There are also the fibroblasts, mast cells and dendritic cells, pilosebaceous units (hair follicles and associated sebaceous glands), eccrine sweat and apocrine glands in dermis. The hypodermis (subcutaneous tissue) plays supporting role to connect the skin to underlying muscle like an anchor [10].

As seen in Figure 1, the potential penetration pathways of drug molecules across the stratum corneum are intercellular, transcellular and/or transappendageal routes [11]. The intercellular route has been considered as the principal penetration pathway of drug molecules, which is defined as transport of drugs across intercellular lamellar 
lipid bilayers. The transcellular route signifies directly to passage of drugs across keratinocytes and intercellular lamellar lipid bilayers to deeper skin layers. These pathways are ascendant for penetration of relatively small $(<500 \mathrm{Da})$ and moderately lipophilic $(\log \mathrm{p}=1-3)$ molecules [12], thus the penetration of highly lipophilic and large molecules through the stratum corneum is limited. In this respect, penetration of highly lipophilic and relatively high-molecular-weight drug molecules into the skin via nanocarriers has gained most of the interest in recent years.

The appendageal pathways (hair follicles, sweat glands and sebaceous glands) (Figure 1), called as shunt route, act in the transport of large and water-soluble drugs. This transport route comprises only $0.1 \%$ of the area of skin surface. On the other hand, in the last decades, shunt route has taken a great attention for nano-based formulations due to its a potential depot effect, high surface area via the hair follicles and extending deep into the skin tissues up to $2000 \mu \mathrm{m}[5,13]$.

\section{Nanocarrier-based carrier systems for dermal drug delivery}

In recent years, dermal drug delivery via nanocarriers has been extensively investigated due to their potential of improving cutaneous drug delivery and targeting of active ingredients into pilosebaceous units [7,13]. Numerous types of nanocarriers including polymeric nanoparticles, micelles, dendrimers, lipid-based carriers (SLNs, NLCs, vesicular systems), microemulsions, nanoemulsions, nanocrystals, nanofibers, nanosponges, nanogels, inorganic and metal nanoparticles such as quantum dots and gold nanoparticles have been widely explored to improve skin delivery of drugs [14-20]. In this review, we opted to examine polymeric-based nanocarriers and lipid-based nanocarriers due to the abundance of research studies related to enhanced penetration and targeting of drugs into the skin. In particular, these nanocarriers that penetrate into the hair follicles have drawn a great attention in the treatment of skin diseases such as melanoma, squamous cell carcinoma, eczema, atopic dermatitis, psoriasis, inflammation, pain, fungal infection, acne and alopecia (Tables $1 \& 2$ ), as well as vaccination, protein, peptide and gene delivery into the skin $[21,22]$.

In the present review, the dispositions and penetration enhancement mechanisms of polymeric nanoparticles, dendrimers, polymeric micelles, lipid-based carriers and vesicular carriers are discussed with handling the studies published in the last 10 years. Moreover, the research studies in which have explained the interaction of polymeric and lipid-based nanocarriers with skin models are mainly taken into consideration in the discussion.

\section{Polymeric nanocarriers}

In dermal drug delivery, the polymeric nanocarriers consist of nanoparticles (nanospheres, nanocapsules and lipidbased nanocapsules), micelles, dendrimers, nanofibers, nanosponges, nanogels, etc. have attracted attention due to their some superiorities over lipid nanocarrier systems such as high physical stability (oxidation observed because of lipid material used in their content) and relatively small particle size. However, some of these carries such as dendrimers do not have enough toxicological assessments.

The advantages and disadvantages of the polymeric nanocarriers are given in Table 3 . The structures of polymeric nanoparticles, polymeric micelles and dendrimers are depicted in Figure 2. The characteristic features of polymeric nanocarriers are summarized in Table 1.

\section{Fundamentals of polymeric nanocarriers}

The polymeric nanoparticles in which the drug is dissolved, entrapped, encapsulated or attached to a matrix/membrane polymer are divided into three main groups: nanospheres with matrix structure, nanocapsules and lipid-based nanocapsules with the membrane structure [73,74] (Figure 2). These carriers are mostly prepared with either natural polymers such as chitosan, cellulose derivatives, alginate, gelatine or synthetic polymers (biodegradable aliphatic polyesters such as polylactides [PLA], poly(f-caprolactone) [PCL] and poly(lactide-co-glycolide) copolymers and nondegradable polymers such as polyacrylates, methyl methacrylate and polystyrene) $[20,26,27,32]$ (Table 1). However, the achievement of reproducible data and drug release in a controlled manner via polymeric nanoparticles consisted of natural polymers is relatively hard due to their variable purity and lack of batch-to-batch consistency [75]. Thus, we only focused the research studies performed on polymeric nanoparticles composed of synthetic polymers in this review.

The polymeric nanoparticles are between 20 and $1500 \mathrm{~nm}$ in particle size and mostly negative charged (Table 1) in this review article, they have unique advantages such as drug release in controlled and sustained manner, numerous 
Table 1. Localization in the skin and characteristics of some polymeric nanocarriers

\begin{tabular}{|c|c|c|c|c|c|c|}
\hline & Size $(n m)$ & $\begin{array}{l}\text { Surface } \\
\text { charge }(m V)\end{array}$ & Drug/labeled molecule & Materials used & Localization in the skin & Ref. \\
\hline \multirow[t]{8}{*}{ Polymeric nanoparticles } & $20-200 \mathrm{~nm}$ & n.d. & Fluorescein & Polystyrene & $\begin{array}{l}\text { The most superficial layers (a } \\
\text { depth of } 2-3 \mu \mathrm{m} \text {, of stratum } \\
\text { corneum) }\end{array}$ & [23] \\
\hline & $20-200 \mathrm{~nm}$ & n.d. & $\begin{array}{l}\text { Fluorescein } \\
5 \text {-isothiocyanate }\end{array}$ & Polystyrene & Furrows and follicular openings & [14] \\
\hline & $90-630 \mathrm{~nm}$ & $16-36 \mathrm{mV}$ & Nile Red & $\begin{array}{l}\text { Cellulose acetate } \\
\text { butyrate/polycaprolactone } \\
\text { /polystyrene }\end{array}$ & $\begin{array}{l}\text { Lipid domains between stratum } \\
\text { corneum } \\
\text { corneocytes and follicular } \\
\text { openings }\end{array}$ & [24] \\
\hline & $40-1500 \mathrm{~nm}$ & n.d. & - & FluoSphere ${ }^{\circledR} /$ Fluoresbrite ${ }^{\mathrm{TM}}$ & Follicular openings & [25] \\
\hline & $320 \mathrm{~nm}$ & n.d. & Sodium fluorescein & PLGA & $\begin{array}{l}\text { Hair follicles after massage } \\
\text { application }\end{array}$ & [26] \\
\hline & $106-257 \mathrm{~nm}$ & -14 to $-10 \mathrm{mV}$ & Clobetasol propionate & $\begin{array}{l}\text { Rhodamin B-labeled-poly }(\varepsilon- \\
\text { caprolactone), polysorbate } \\
80, \text { capric-caprylic } \\
\text { triglyceride/sorbitan } \\
\text { monostearate }\end{array}$ & Hair follicles & [27] \\
\hline & $50-1000 \mathrm{~nm}$ & -2 to $-5 \mathrm{mV}$ & Betamethasone/Nile Red & $\begin{array}{l}\text { Ethyl cellulose, polyvinyl } \\
\text { alcohol }\end{array}$ & $\begin{array}{l}\text { In inflamed skin hair follicles and } \\
\text { sebaceous glands, On hairs, } \\
\text { corneocytes borders, skin furrows } \\
\text { and around hair follicles in } \\
\text { healthy skin }\end{array}$ & [29] \\
\hline & $227-674 \mathrm{~nm}$ & -11 to $-32 \mathrm{mV}$ & Retinyl acetate & $\begin{array}{l}\text { Ethyl cellulose, poly(ethylene } \\
\text { glycol)-4-methoxy } \\
\text { cinnamoylphthaloylchitosan }\end{array}$ & $\begin{array}{l}\text { Hair follicles (drug delivery into } \\
\text { dermis and away from the hair } \\
\text { follicles) }\end{array}$ & [30] \\
\hline \multirow[t]{3}{*}{$\begin{array}{l}\text { Combination with } \\
\text { penetration enhancers }\end{array}$} & $78-180 \mathrm{~nm}$ & -26 to $-39 \mathrm{mV}$ & $\begin{array}{l}\text { Dehydroepiandrosterone/ } \\
\text { dye }\end{array}$ & $\begin{array}{l}\text { PLGA, Solutol HS 15, Tween }{ }^{\circledR} \\
\text { II80 }\end{array}$ & Internal viable skin layers & [31] \\
\hline & $70-300 \mathrm{~nm}$ & -12 to $0 \mathrm{mV}$ & - & $\begin{array}{l}\text { Fluoresceinamine grafted } \\
\text { PLGA, polyvinyl alcohol }\end{array}$ & $\begin{array}{l}\text { Up to depth of } 55 \mu \mathrm{m} \\
\text { inflammatory skin }\end{array}$ & [34] \\
\hline & $153-217 \mathrm{~nm}$ & 5-17 mV & $\begin{array}{l}\text { Spantide II, } \\
\text { ketoprofen/DiO/DID dye }\end{array}$ & PLGA, chitosan, oleic acid & Up to depth of $240 \mu \mathrm{m}$ & [35] \\
\hline \multirow[t]{4}{*}{ Polymeric micelles } & $25-52 \mathrm{~nm}$ & n.d. & $\begin{array}{l}\text { Fluorescein-labeled- } \\
\text { cyclosporin } \\
\text { A }\end{array}$ & $\begin{array}{l}\text { Nile } \\
\text { Red-labeled-mPEG-dihex-PLA }\end{array}$ & $\begin{array}{l}\text { Between corneocytes and in the } \\
\text { intercluster regions }\end{array}$ & [36] \\
\hline & $70-163 \mathrm{~nm}$ & n.d. & $\begin{array}{l}\text { Clotrimazole, econazole } \\
\text { nitrate, } \\
\text { fluconazole/fluorescein }\end{array}$ & mPEG-dihex-PLA & Hair follicles & [15] \\
\hline & $51-106 \mathrm{~nm}$ & n.d. & $\begin{array}{l}\text { Tacrolimus/fluorescein, } \\
\text { DiO }\end{array}$ & $\begin{array}{l}\text { Nile } \\
\text { Red-labeled-mPEG-dihex-PLA }\end{array}$ & Hair follicles & [37] \\
\hline & $72-106 \mathrm{~nm}$ & n.d. & Retinoic acid & mPEG-dihex-PLA & Pilosebaceous unit & [38] \\
\hline \multirow[t]{2}{*}{ Dendrimers } & n.d. & n.d. & Methoxy psoralene & $\begin{array}{l}\text { Fluorescein-conjugated } \\
\text { PAMAM }\left(G_{3} \text { and } G_{4}\right)\end{array}$ & $\begin{array}{l}\text { Release into epidermis and } \\
\text { dermis of drug and increased } \\
\text { transdermal flux }\end{array}$ & [39] \\
\hline & $11-18 \mathrm{~nm}$ & -13 to $26 \mathrm{mV}$ & - & $\begin{array}{l}\text { Fluoroisothiocynate-labeled } \\
\text { PAMAM }\left(\mathrm{G}_{2}-\mathrm{G}_{6} \mathrm{NH}_{2}\right. \\
\left.\mathrm{G}_{3.5}-\mathrm{COOH} \text { and } \mathrm{G}_{4}-\mathrm{OH}\right)\end{array}$ & $\begin{array}{l}\text { Furrows, intercellular lipids and } \\
\text { hair follicles (in case of passive } \\
\text { and ionthophosis) }\end{array}$ & [40] \\
\hline
\end{tabular}


Table 1. Localization in the skin and characteristics of some polymeric nanocarriers (cont.).

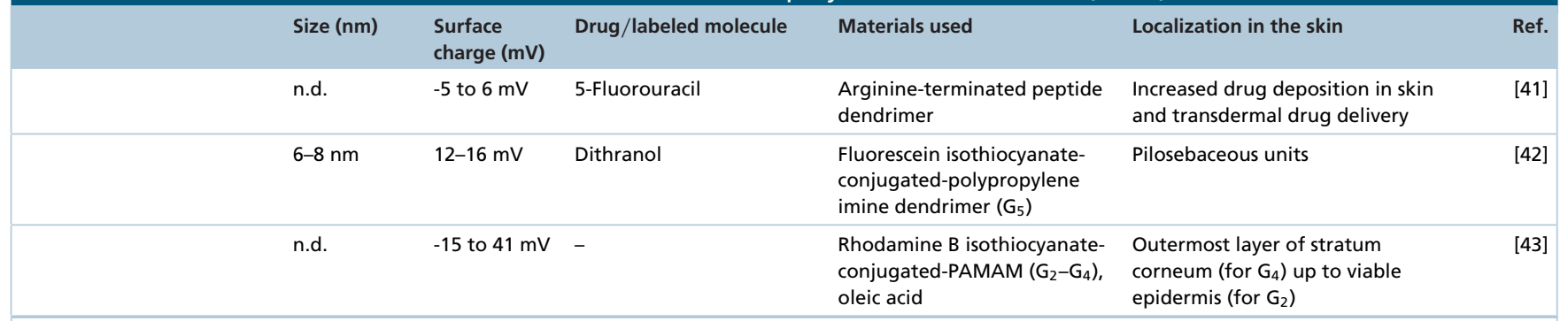

HPMC: Hydroxypropyl methylcellulose; MEH-PPV: Poly[2-methoxy-b-(2 ethylhexyloxy)-1,4-phenylenevinylene]; mPEG-dihex-PLA: Methoxy-poly(ethylene glycol)-hexyl-substituted polylactide; n.d.: Not determined; PAMAM: Poly(amidoamine); PLGA: Polylactide-co-glycolide; PMMA: Poly[methyl methacrylate-co-(fluorecein-o-methacrylate)].

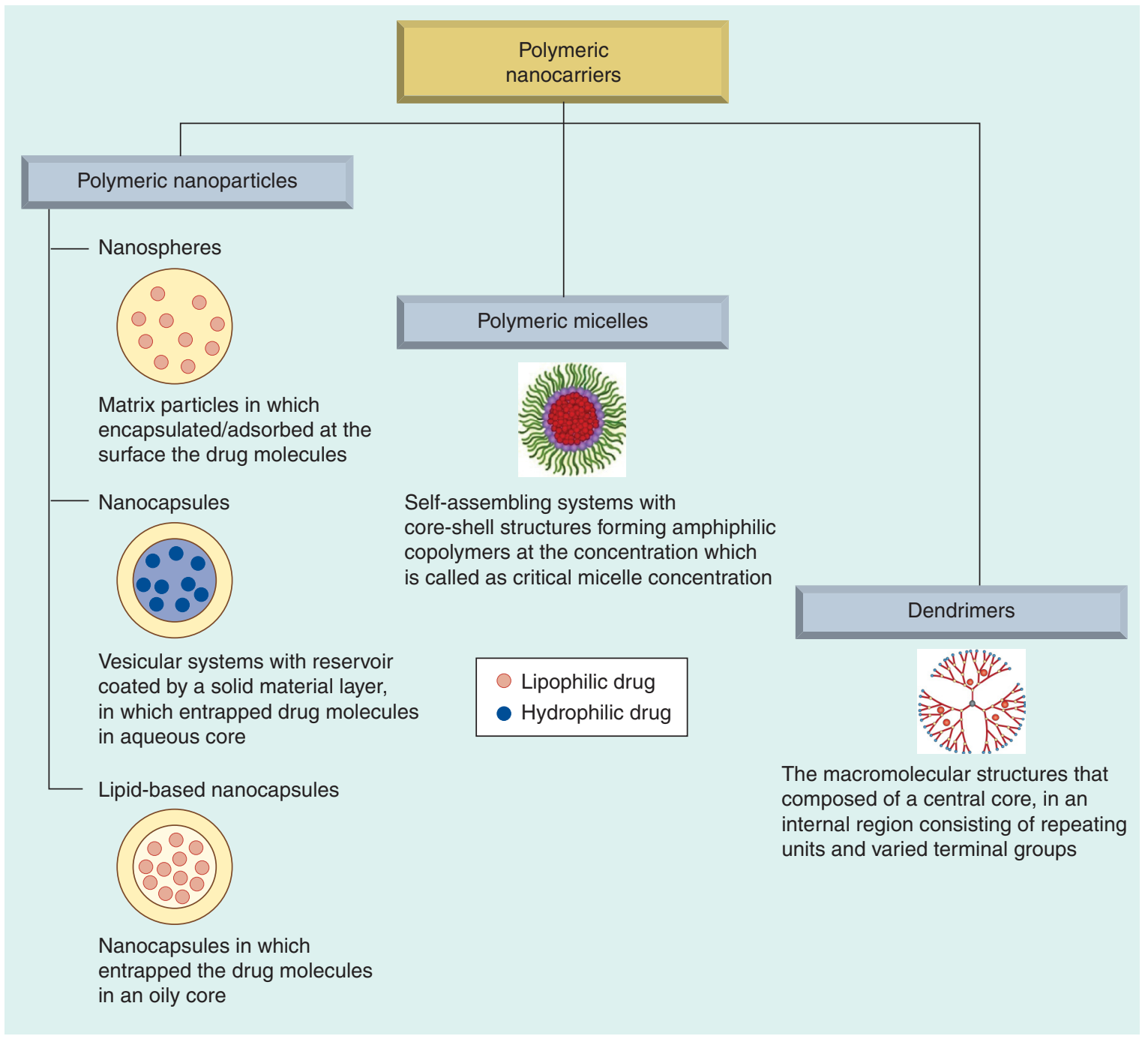

Figure 2. The structures of polymeric nanocarriers.

of preparation methods. But, they also have some limitations such as problems in scale-up process and lack of their toxicity assessments [72].

The polymeric micelles have gained a great attention with their abilities, which increase the solubility of highly lipophilic drugs in recent years. These carriers self-assemble from amphiphilic block copolymers at/above specific polymer concentration called as 'critical micelle concentration' to decrease surface-free energy in an aqueous 
Table 2. Drug delivery into the skin and characteristics of some lipid nanocarriers

\begin{tabular}{|c|c|c|c|c|c|}
\hline & Size $(\mathrm{nm})$ & $\begin{array}{l}\text { Surface charge } \\
(\mathrm{mV})\end{array}$ & Drug/labeled molecule & Drug delivery into the skin & Ref. \\
\hline \multirow[t]{10}{*}{ Lipid-based nanoparticles } & $150-212 \mathrm{~nm}$ & n.d. & Betamethasone-17-valerate & Stratum corneum & [44] \\
\hline & $762 \mathrm{~nm}$ & $-30 \mathrm{mV}$ & $\mathrm{TiO}_{2}$ & $\begin{array}{l}\text { The deepest stratum corneum layers (up } \\
\text { to the corneum compactum) }\end{array}$ & [45] \\
\hline & $166-1227 \mathrm{~nm}$ & -7 to $-36 \mathrm{mV}$ & $\begin{array}{l}\text { Econazole nitrate/Rhodamine } \\
\text { Red }\end{array}$ & Up to the stratum basale & [46] \\
\hline & $30-49 \mathrm{~nm}$ & n.d. & Idebenone & Upper skin layers and epidermis & [47] \\
\hline & $140-220 \mathrm{~nm}$ & $-12 \mathrm{mV}$ & Adapalene/FITC & Epidermis & [48] \\
\hline & $141-203 \mathrm{~nm}$ & -6 to $-50 \mathrm{mV}$ & Coenzyme Q10 & Epidermis & [49] \\
\hline & $172 \mathrm{~nm}$ & $-32 \mathrm{mV}$ & Roxithromycin/RBHE & Pilosebaceous units & [50] \\
\hline & $177 \mathrm{~nm}$ & $-54 \mathrm{mV}$ & $\begin{array}{l}\text { Diphencyprone, minoxidil/Nile } \\
\text { Red }\end{array}$ & Hair follicles & [51] \\
\hline & $305-358 \mathrm{~nm}$ & $30-36 \mathrm{mV}$ & Genistein & Deeper skin layers (especially for NLC) & [52] \\
\hline & $137-161 \mathrm{~nm}$ & -28 to $-45 \mathrm{mV}$ & $\begin{array}{l}\text { All-trans-retinoic } \\
\text { acids/Rhodamine B }\end{array}$ & $\begin{array}{l}\text { Rhodamin B delivery up to depth of } \\
30 \mu \mathrm{m}(10 \% \text { limonen SLN) and } 75 \mu \mathrm{m} \\
(10 \% \text { limonen NLC) }\end{array}$ & [53] \\
\hline \multicolumn{6}{|l|}{ Vesicular nanocarriers } \\
\hline \multirow[t]{3}{*}{ Liposomes } & $77-88 \mathrm{~nm}$ & -6 to $-10 \mathrm{mV}$ & Calcipotriol & Epidermis of diseased skin & [54] \\
\hline & $115-149$ nm & 0 to $-4 \mathrm{mV}$ & Vitamin K1 & Epidermis and dermis & [55] \\
\hline & n.d. & n.d. & $\gamma$-Reductase inhibitor & $\begin{array}{l}\text { Flank organs including sebaceous } \\
\text { glands, hair follicles and melanocytes } \\
\text { clumps }\end{array}$ & [56] \\
\hline \multirow[t]{4}{*}{$\begin{array}{l}\text { Combination with } \\
\text { penetration enhancer }\end{array}$} & $80-140 \mathrm{~nm}$ & -7 to $3 \mathrm{mV}$ & Methotrexate & $\begin{array}{l}\text { Epidermis and dermis layers (with oleic } \\
\text { acid) }\end{array}$ & [57] \\
\hline & $116-164 \mathrm{~nm}$ & -47 to $-69 \mathrm{mV}$ & Tretinoin & $\begin{array}{l}\text { Deeper layers of the epidermis (in } \\
\text { presence of penetration enhancer) }\end{array}$ & [58] \\
\hline & $140-195 \mathrm{~nm}$ & -52 to $-58 \mathrm{mV}$ & Minoxidil & $\begin{array}{l}\text { Upper skin layers (in presence of } \\
\text { penetration enhancer) }\end{array}$ & [59] \\
\hline & $87-146 \mathrm{~nm}$ & -53 to $-78 \mathrm{mV}$ & $\begin{array}{l}\text { Diclofenac/ } \beta \text {-carotene } / 5(6) \text { - } \\
\text { carboxyfluorescein }\end{array}$ & $\begin{array}{l}\text { Stratum corneum, epidermis, dermis } \\
\text { and transdermal delivery (diversity by } \\
\text { amount of penetration enhancer) }\end{array}$ & [60] \\
\hline \multirow[t]{3}{*}{ Niosomes } & $299-402 \mathrm{~nm}$ & n.d. & Resveratrol & Epidermis and dermis & [61] \\
\hline & $124-752 \mathrm{~nm}$ & n.d. & Ellagic acid & Up to depth of $120 \mu \mathrm{m}$ & [62] \\
\hline & $156-245 \mathrm{~nm}$ & -48 to $-57 \mathrm{mV}$ & $\begin{array}{l}\text { Tretinoin/5(6)- } \\
\text { carboxyfluorescein/Rhodamine- } \\
\text { PE }\end{array}$ & $\begin{array}{l}\text { Skin appendages (for niosomes with } \\
\text { labrasol) }\end{array}$ & [63] \\
\hline \multirow[t]{5}{*}{ Transfersomes } & $39 \mathrm{~nm}$ & $-10 \mathrm{mV}$ & $\begin{array}{l}\text { Fluorescein } \\
\text { sodium/Rhodamine-PE }\end{array}$ & $\begin{array}{l}\text { Stratum corneum and hair roots (in case } \\
\text { of } 1 \% \text { D-limonene) }\end{array}$ & [64] \\
\hline & $106-145 \mathrm{~nm}$ & -1 to $-11 \mathrm{mV}$ & Amphotericin B & Stratum corneum and viable epidermis & [65] \\
\hline & $131 \mathrm{~nm}$ & $-6 \mathrm{mV}$ & Tretinoin/Nile Red & $\begin{array}{l}\text { Especially stratum corneum, viable } \\
\text { epidermis and dermis }\end{array}$ & [66] \\
\hline & $115-122 \mathrm{~nm}$ & -23 to $27 \mathrm{mV}$ & Aminolevulinic acid & Epidermis and dermis & [67] \\
\hline & $307-664$ nm & -19 to $-33 \mathrm{mV}$ & $\begin{array}{l}\text { Resveratrol, } \\
\text { 5-fluorouracil/Rhodamine-DHPE }\end{array}$ & Deeper strata of the skin & [68] \\
\hline \multirow[t]{3}{*}{ Ethosomes } & $130-137$ nm & $-19 \mathrm{mV}$ & Vitamin E, caffeine & Stratum corneum, epidermis, dermis & [69] \\
\hline & $203-257$ nm & -56 to $-84 \mathrm{mV}$ & Clotrimazole/Rhodamine B & Epidermis & [70] \\
\hline & $176-269 \mathrm{~nm}$ & -49 to $-75 \mathrm{mV}$ & $\begin{array}{l}\text { Econazole nitrate/Rhodamine } \\
\text { Red }\end{array}$ & $\begin{array}{l}\text { The last layer of epidermis (stratum } \\
\text { basale) }\end{array}$ & [71] \\
\hline
\end{tabular}

FITC: Fluorescein isothiocyanate; n.d.: Not determined; NLC: Nanostructured lipid carrier; RBHE: Rhodamine B hexyl ester perchlorate; Rhodamine-DHPE: Rhodamine B-1,2dihexadecanoyl-sn-glycero-3-phosphoethanolamine triethylammonium salt.

medium. The amphiphilic block copolymers comprise a hydrophilic polymer such as polyethylene glycol (PEG) and a hydrophobic polymer such as PLA, PCL, etc. The hydrophobic core gives rise to both of drug solubility and depot effect while the hydrophilic shell generates stabilizing due to their core-shell structures in aqueous medium [76,77] (Figure 2). Polymeric micelles also have the other superiorities of polymeric nanocarrier such as 


\begin{tabular}{|c|c|c|}
\hline Nanocarriers & Advantages & Disadvantages \\
\hline \multirow[t]{8}{*}{ Polymeric nanoparticles } & Drug release in controlled and sustained manner & Difficulty for their scale-up \\
\hline & Incorporation of hydrophilic and hydrophobic drugs & Insufficient of toxicological assessment in the literature \\
\hline & Tunable chemical and physical properties & \\
\hline & Use of a lot of biodegradable materials when desired & \\
\hline & $\begin{array}{l}\text { Existence of } \mathrm{pH} \text {, enzymatic, hydrolysis, etc., sensitive } \\
\text { properties when preferred proper polymers }\end{array}$ & \\
\hline & Reproducible data when used synthetic polymers & \\
\hline & Higher stability than lipid based ones & \\
\hline & Being many methods to prepare them & \\
\hline \multirow[t]{4}{*}{ Polymeric micelles } & Increasing solubility of highly lipophilic drugs & Use only for lipophilic drugs \\
\hline & Tunable chemical and physical properties & Low drug-loading capacity \\
\hline & Drug release in controlled manner & Dependency of critical micelle concentration \\
\hline & Protecting drug from environmental conditions & \\
\hline \multirow[t]{5}{*}{ Dendrimers } & Increasing solubility of highly lipophilic drugs & Not a good candidate carrier for hydrophilic drugs \\
\hline & Tunable chemical and physical properties & Cellular toxicity \\
\hline & Multiple functional groups for targeted drug delivery & $\begin{array}{l}\text { Elimination and metabolism depending on the generation } \\
\text { and materials }\end{array}$ \\
\hline & Covalently associating drugs & High cost for their synthesis \\
\hline & Acting like solubility enhancers & \\
\hline
\end{tabular}

tunable chemical and physical properties [7] (Table 3). However, they have some limitations including dependence of critical micelle concentrations and low drug-loading capacity [78,79].

The dendrimers have been widely explored for diagnostic imaging and drug delivery in recent years. They are unimolecular, monodisperse, synthetic polymers (< generally $15 \mathrm{~nm}$ ) with highly branched structures. These molecules have a layered structure that composed of a central core, an internal region consisting of repeating units and varied terminal groups that result in 3D and fractal architecture. Dendrimers exhibit relatively uniform shapes, sizes and molecular weights [80] (Figure 2). Although they might have cellular toxicity and high cost for their synthesis, etc., dendrimers are considered as potential carriers for targeted drug delivery due to multiple functional groups in their structure [72] (Table 3).

\section{The potential penetration enhancement mechanisms of polymeric nanocarriers}

It is generally accepted that the intact penetration of polymeric nanoparticles across the stratum corneum is negligible [6]. It has been shown that the polymeric nanoparticles applied onto the skin have generally localized in stratum corneum surface, furrows and openings of hair follicles (infundibulum) $[14,23,24,81]$. Thus, these nanocarriers can generate a depot effect in these skin regions, resulting in high drug concentrations. Then, the drug is released in a sustained manner into viable skin layers such as epidermis and dermis (Table 4). Campbell et al. [23] showed that polystyrene nanoparticles (20-200 $\mathrm{nm}$ ) existed only 2-3 $\mu \mathrm{m}$ in depth at the superficial layers (Table 1 ) and the nanoparticles did not reach into deeper skin layers despite removing partly of the stratum corneum by tape stripping. They underlined that polymeric nanoparticles would not be reached into viable epidermis and deeper tissues. On the other hand, it has been proved that the polymeric nanoparticles have mostly accumulated in hair follicles and furrows on the skin surface based on the confocal laser scanning microscopy (CLSM) images [14]. The findings of Abdel-Mottaleb et al. [82] verified that the polymeric nanoparticles exhibited fourfold greater accumulation in the skin compared with lipid-based nanoparticles. However, it has been reported that the drug penetration amount from nanoparticles into the skin could be affected by individual characteristics of these carriers such as surface properties, rigidity, hydrophobicity and particle size (Table 1). In addition, higher drug delivery was observed in the case of smaller particle size of nanocarriers due to increased exposure area of the particles into the skin while less drug delivery with the increase in hydrophobicity of nanocarriers [24]. Vogt et al. [25] also viewed that only $40 \mathrm{~nm}$ of fluorescence polystyrene nanoparticles penetrated into follicular epithelium and larger particles than that of $40 \mathrm{~nm}$ ones remained in the entrance of hair follicles. Thus, the polymeric nanoparticles could be considered as appropriate carriers, which enable a sustained and localized drug release on the skin surface as a drug reservoir. 
Table 4. Potential skin penetration enhancement mechanisms of nanocarriers.

\begin{tabular}{|c|c|c|c|c|c|c|}
\hline & \multicolumn{3}{|c|}{ Polymeric nanocarriers } & \multicolumn{3}{|c|}{ Lipid nanocarriers } \\
\hline & \multirow{2}{*}{$\begin{array}{l}\text { Polymeric } \\
\text { nanoparti- } \\
\text { cles }\end{array}$} & \multirow{2}{*}{$\begin{array}{l}\text { Polymeric } \\
\text { micelles }\end{array}$} & \multirow[t]{2}{*}{ Dendrimers } & \multirow{2}{*}{$\begin{array}{l}\text { Lipid-based } \\
\text { nanocarriers } \\
\text { (SLNs and } \\
\text { NLCS) }\end{array}$} & \multicolumn{2}{|c|}{ Vesicular nanocarriers } \\
\hline & & & & & Liposomes & $\begin{array}{l}\text { Flexible vesicular } \\
\text { nanocarriers } \\
\text { (niosomes, } \\
\text { transfersomes and } \\
\text { ethosomes) }\end{array}$ \\
\hline Increase in thermodynamic activity of the drug & $\checkmark$ & $\checkmark$ & $\checkmark$ & $\checkmark$ & $\checkmark$ & $\checkmark$ \\
\hline Disposition on the stratum corneum surface & $\checkmark$ & & & $\checkmark$ & $\checkmark$ & \\
\hline Localization of in furrows & $\checkmark$ & $\checkmark$ & $\checkmark$ & & & \\
\hline $\begin{array}{l}\text { Deposition of in pilosebaceous units including } \\
\text { hair follicles }\end{array}$ & $\checkmark$ & $\checkmark$ & $\checkmark$ & $\checkmark$ & $\checkmark$ & $\checkmark$ \\
\hline Acting as penetration enhancer & & $\begin{array}{l}\checkmark \\
\text { Unimers- } \\
\text { forming } \\
\text { micelles }\end{array}$ & $\checkmark$ & & & $\checkmark$ \\
\hline $\begin{array}{l}\text { Film formation on the skin surface and occlusive } \\
\text { effect }\end{array}$ & & & & $\checkmark$ & & \\
\hline Fusion onto the stratum corneum surface & & & & & & $\checkmark$ \\
\hline Penetration enhancing effect of ethanol & & & & & & $\begin{array}{l}\checkmark \\
\text { For ethosomes }\end{array}$ \\
\hline
\end{tabular}

NLC: Nanostructured lipid carrier; SLN: Solid-lipid nanoparticle

The potential of polymeric micelles for the dermal drug delivery has been widely investigated in recent years $[15,36-$ 38,83-85]. Regarding potential penetration enhancement of polymeric micelles, early studies indicated that fluorescein loaded into micelles reached up to the depth of $40 \mu \mathrm{m}$ via hair follicles. Additionally, the penetration of the antifungal drug into the porcine and human skin from polymeric micelles was found significantly higher than that of its commercial liposomal formulation [15]. Then, same researchers reported that Nile Red-labeled mPEG-dihexPLA micelles were localized between corneocytes and in the intercluster regions (furrows) via CLSM images. They also indicated that fluorescein-labeled cyclosporin A in the micelles penetrated into deeper layers of the skin by releasing drug from the micelles in the intercluster regions, which might be considered as a potential penetration route for cutaneous drug delivery through the micelles [36] (Table 4). The same group researchers also reported that tacrolimus loaded in mPEG-dihex-PLA micelles was delivered higher amounts of the drug into the stratum corneum, viable epidermis and upper dermis compared with its commercial ointment product. However, they suggested that the copolymer could not pass across the stratum corneum but localized into follicular ducts [37] (Table 4). Similarly, it was indicated by same researchers that retinoic acid-loaded mPEG-dihex-PLA micelles caused to twofold higher drug accumulation in pilosebaceous units using the punch biopsy method [38]. Also, the accumulation of the polymeric micelles into hair follicles has intensely gained interest in recent years. So that, antiacne drugs such as all transretinol [83], retinoic acid [38], benzoyl peroxyde [84]-loaded micelles were prepared using poly(lactide)-block-poly(ethylene glycol) (PLA-b-PEG)/poly(caprolactone)-block-poly(ethylene glycol) (PCL$b$-PEG), mPEG-dihex-PLA and Pluronic ${ }^{\circledR}$ F127 copolymer, respectively.

In the last published study, Smejkalová et al. [85] showed that polymeric micelles either generated from fluorescentlabeled hydrophobized HA or loaded with Nile Red highly accumulated in epidermis and dermis layers of the based on CLMS images. However, Förster resonance energy transfer pair dyes which loaded in the micellar core made this extraordinary accumulation clarify. The observed data suggested that polymeric micelles might start to decay with increasing the skin depth and then, hydrophobic drug could reach to dermis preferentially via transcellular pathway. Interestingly, this research revealed that modified HA by itself penetrated into deeper skin layer. Although it was not mentioned in this paper, intensively fluorescence accumulation into hair follicles has been observed in CLMS images. Thus, it might be said that drug-loaded polymeric micelles mostly localize in hair follicles and furrows, then the micelles could decay with time in the skin. Consequently, the drug penetration into viable skin layers increases due to drug release from the micelles. In addition, amphiphilic copolymers could exhibit penetration enhancer effect due to their surfactant properties. Here, polymeric micelles could also act as a penetration enhancer following the micelles decay in the skin (Table 4). 


\begin{tabular}{|c|c|c|}
\hline Nanocarriers & Advantages & Disadvantages \\
\hline \multirow{5}{*}{$\begin{array}{l}\text { Lipid-based nanocarriers (SLNs } \\
\text { and NLCs) }\end{array}$} & Producing on large industrial scale & Low drug loading for SLNs \\
\hline & $\begin{array}{l}\text { Low toxicity due to their biocompatible and } \\
\text { biodegradable components and absence of organic } \\
\text { solvent(s) in their process }\end{array}$ & Risk of gelation for SLNs \\
\hline & Incorporation of lipophilic and hydrophilic drugs & $\begin{array}{l}\text { Drug expulsion during storage cause by lipid } \\
\text { polymorphism for SLNs }\end{array}$ \\
\hline & Protecting drug from environmental conditions & \\
\hline & Low cost compared with liposomes & \\
\hline \multicolumn{3}{|l|}{ Vesicular nanocarriers } \\
\hline \multirow[t]{4}{*}{ Liposomes } & $\begin{array}{l}\text { Encapsulating both of hydrophilic and lipophilic } \\
\text { drugs in their different phases }\end{array}$ & $\begin{array}{l}\text { Poor stability due to phospholipids of predisposition } \\
\text { to oxidative degradation }\end{array}$ \\
\hline & Protecting drug from environmental conditions & Requirement of special storage \\
\hline & Having good biocompatibility and biodegradability & Limited penetration in the skin \\
\hline & Low toxicity & \\
\hline \multirow[t]{5}{*}{$\begin{array}{l}\text { Flexible vesicular nanocarriers } \\
\text { (niosomes, transfersomes and } \\
\text { ethosomes) }\end{array}$} & Incorporation of lipophilic and hydrophilic drugs & $\begin{array}{l}\text { Obligation of emulsifiers (edge activators) high purity } \\
\text { to avoid risk of skin irritation and toxicity for } \\
\text { transfersomes }\end{array}$ \\
\hline & $\begin{array}{l}\text { Higher encapsulation efficiency and stability in } \\
\text { compared with liposomes }\end{array}$ & $\begin{array}{l}\text { Skin irritation due to high concentration of alcohol } \\
\text { for ethosomes }\end{array}$ \\
\hline & $\begin{array}{l}\text { Simple and inexpensive to manufacture in compared } \\
\text { with liposomes }\end{array}$ & \\
\hline & $\begin{array}{l}\text { Ability of intactly transport of across stratum } \\
\text { corneum due to their soft and malleable } \\
\text { characteristics }\end{array}$ & \\
\hline & Enhanced penetration and permeation & \\
\hline
\end{tabular}

The localization of the dendrimers in the furrows and pilosebaceous units including hair follicles has also observed [40,42] (Table 4). However, the penetration and permeation of dendrimers into and through the skin are a controversial issue and it has thought that it could depend on their size/generation (G), hydrophobicity, surface charge and concentration $[41,43,86]$ (Table 1). It was reported that dendrimers bounded with oleic acid as, terpenes, fatty acids permeation enhancers, chemically surface-modified dendrimers by acetylation or carboxylation [43] increased only drug penetration up to the viable epidermis, as charged peptide dendrimers with arginine [41], and the some poly(amidoamine) dendrimers [39] produced transdermal drug delivery (Table 1). Sun et al. [87] discussed three different possible enhancement mechanisms of drug penetration through dendrimers. In the first approach, it is considered that the drugs loaded to dendrimers cross the skin with increased drug concentration in the carrier, but not dendrimers. In the second approach, the passage of the dendrimer itself is assumed by interacting between penetration enhancer and the stratum corneum lipids, which results in disruption of skin structural integrity. Thus, the passage of drug across the skin has improved. The last approach is regarded to the localization of drug-dendrimer complexes in the hair follicles and release of the drug at high concentration in hair follicles (Table 4).

The combination of polymeric nanoparticles and physicochemical methods have also been available to increase drug penetration into viable skin layers in the literature [31-35,88-91]. In these studies, the combination of polymeric nanoparticles and penetration enhancers has been on the agenda in recent years [31,33-35]. In the case of these combinations were determined an increase up to $240 \mu \mathrm{m}$ in depth [35] (Table 1). This penetration enhancement might be due to release of drug and penetration enhancer together.

\section{Lipid nanocarriers}

The lipid nanocarriers comprise of lipid-based nanoparticles including SLNs, NLCs and vesicular nanocarriers including liposomes, niosomes, tranfersomes, ethosomes. The structures of lipid-based nanoparticles and vesicular nanocarriers are depicted in Figure 3. The characteristic features of lipid nanocarriers are summarized in Table 2. The advantages and disadvantages of the lipid nanocarriers are given in Table 5. 


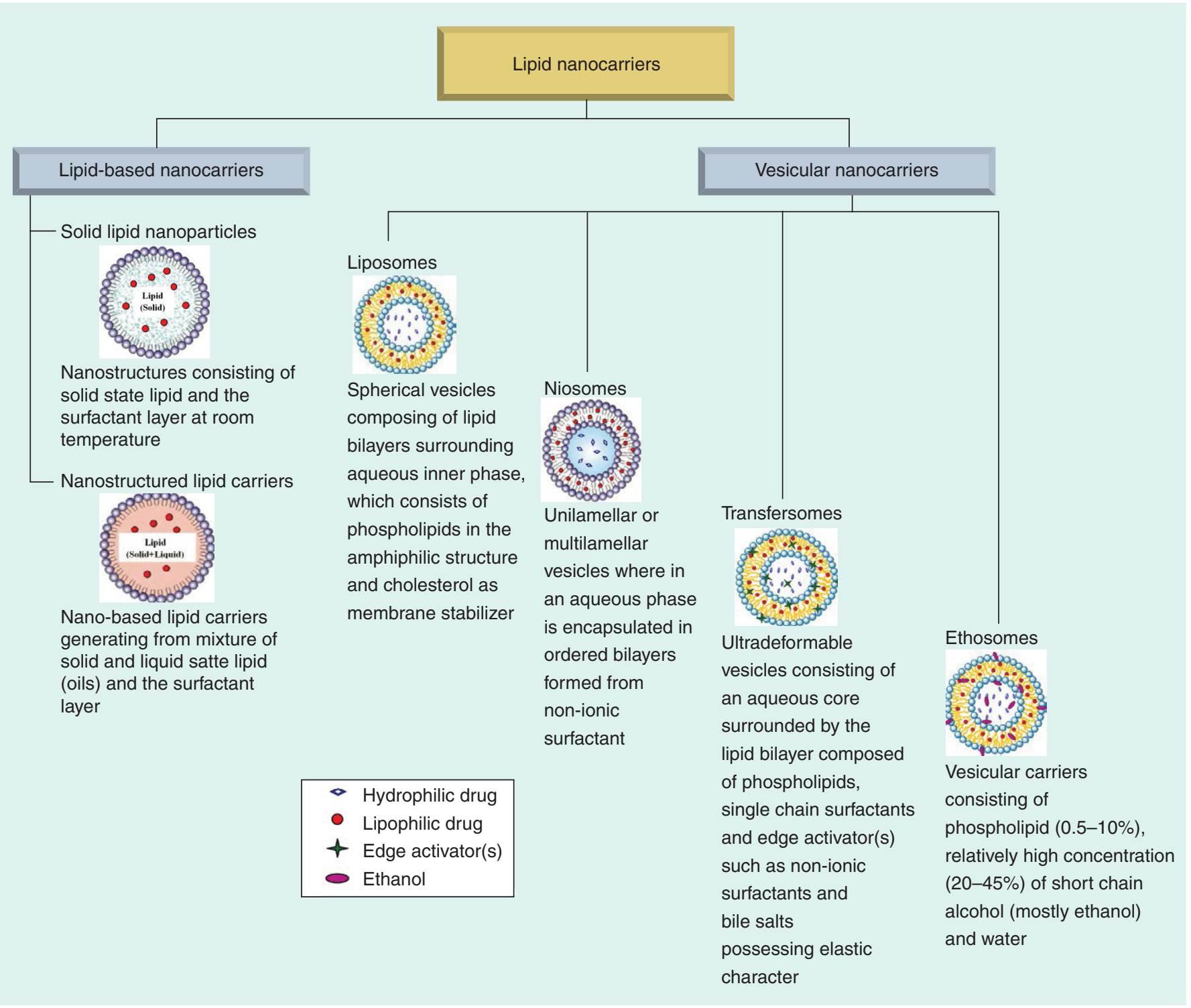

Figure 3. The structures of lipid nanocarriers.

\section{Fundamentals of lipid-based nanocarriers}

Lipid-based nanocarriers are classified as SLNs and NLCs. SLNs are prepared from solid lipid(s) that is/are in solid form at body and room temperatures and are mostly stabilized by surfactants (Figure 3). SLNs commonly are consisted of glycerol behenate (Compritol 888 ATO), glycerol palmitostearate (Precirol ATO 5), glyceryl tripalmitate (Dynasan 116), stearate or palmitate, cetyl palmitate and glyceryl trimyristate as solid state lipid(s) [9294]. It has assumed that they might be produced on the large industrial scale. Moreover, they have less toxicity due to their biocompatible and biodegradable components and absence of organic solvent(s) in their production process in comparison with polymeric nanoparticles [95] (Table 5). However, they have disadvantages such as low drug loading, risk of gelation, the expulsion of drug from the carrier caused by lipid polymorphism during storage period [4]. Therefore, NLCs prepared from liquid lipid(s) mixed with solid lipid(s), which is/are in solid form at room temperature, and surfactant are generated to overcome the limitations of SLNs (Table 5). NLCs exhibit greater drug penetration across skin due to increased drug solubility in liquid lipid(s) and drug-loading capacity [6]. In the composition of NLCs, medium-chain triglycerides (Miglyol 812, Squalene), triglycerides of caprylic and capric acid (Captex 355) and/or oleic acid using as the liquid state lipid (s) are mostly used [5]. Furthermore, Solutol 
HS15, Cremophor A25, polysorbate 80, etc., are added to the composition to SLNs and NLCs as surfactant between 0.5 and $5 \%[44,82,95]$.

\section{The potential skin penetration-enhancement mechanisms of lipid-based nanocarriers}

Lots of research studies have reported that SLNs and NLCs are proposed to be ideal carriers for epidermal and follicular targeting of drugs in the skin delivery [44-46,48-50] (Table 2). Both SLNs and NLCs could not penetrate through the stratum corneum, but remain in the upper skin layers [44]. The lipid-based nanocarriers form a hydrophobic monolayer film, which generates occlusive effect following their accumulation on the skin surface. Then, the transepidermal water loss reduces, and barrier function of the stratum corneum is protected [4]. As a consequence, compact structure of the stratum corneum loosens due to its hydration and, then drug loaded into the carriers penetrates into the epidermal layers [44] (Table 4). Small particle size, highly crystalline structure and lowmelting lipid components are crucial factors for this mechanism [96]. Moreover, facilitating penetration of lipophilic drugs via SLNs and NLCs might occur during the occlusion due to the interaction between the stratum corneum lipids and nanocarrier lipids. Küchler et al. [97] revealed that platelet structure of SLN(s) deteriorated following their administration onto the skin surface $2 \mathrm{~h}$ later. Similarly, Khurana et al. [98] promoted this finding using the nanocarriers containing cetyl palmitate via differential scanning calorimetry and Fourier transform infrared spectroscopy analysis. Zhang and Smith [99] also demonstrated that this case highly depends on the content of lipid-based nanocarriers.

The lipid-based nanocarriers, which intensively accumulate in the hair follicles could reach into viable skin layers through shunt pathway [51] (Table 4). Wosicka et al. [50] demonstrated that Rhodamine B hexyl ester perchlorate-loaded SLN(s) localized up to $1 \mathrm{~mm}$ of depth in the hair follicles. Furthermore, Lauterbach and Muller-Goymann [100] have proposed that lipids in SLNs and NLCs are similar to sebum lipids in hair follicles, not the stratum corneum lipids. Thus, the hair follicles might act as an effective depot for these carriers. Interestingly, Aljuffali et al. [51] developed nanoparticles called as 'squaticles,' comprised squalene and sebum-derived lipids such as fatty acid esters, to enhance the delivery of minoxidil and diphencyprone into the hair follicles and related to the cells. The follicular uptakes of the drugs via squaticles enhanced compared with their solutions. Furthermore, fluorescence and CLSM images evidenced that the squaticles were localized into the hair follicles and reached to deeper skin layers. Also, the expression of vascular endothelial growth factor was upregulated in dermal papilla cells following application of minoxidil-loaded squaticles to the mice, indicating the squaticles provided targeting of the drug in dermal papilla cells and further follicular drug accumulation.

The other strategy to enhance drug penetration across the skin is the combination of lipid-based nanocarriers and physicochemical methods as seen in polymeric nanocarriers. To enhance drug penetration into viable skin layers, the penetration enhancers such as terpenes were also used with both of SLNs and NLCs. Charoenputtakun et al. [53] prepared Rhodamine B-loaded NLCs with limonene and showed that Rhodamin B reached up to $75 \mu \mathrm{m}$ via combination of NLCs and limonene as up to $30 \mu \mathrm{m}$ via combination of SLNs and limonene (Table 2).

The surface modification of NLCs might be an alternative for enhancing delivery and targeting of NLCs to the skin layers. Singh and colleagues [101-103] revealed that NLCs modified the cell-penetrating peptide (CPP; transactivating transcriptional activator) exhibited greater epidermal localization of a fluorescent probe and celecoxib in comparison with control NLCs formulation. Also, they have suggested that a CPP containing 11 arginines and transactivator of transcription (TAT) peptides have penetration enhancing ability.

\section{Fundamentals of vesicular nanocarriers}

The liposomes, one of the first vesicular systems, are composed of phospholipids and cholesterol, which associate as a double layer (unilamellar vesicles) or multiple double layers (multilamellar vesicles) (Figure 3). The liposomes typically have spherical shape and are between 25 and $5000 \mathrm{~nm}$ in size [104]. They might be ideal candidate due to their lipid composition, which is similar to epidermis [4]. They have other superiorities such as encapsulating both hydrophilic and lipophilic compounds in their different phases, protecting drug from external environment, having good biocompatibility, biodegradability and low toxicity $[4,105]$. However, they have chemically poor stability due to predisposition of phospholipids to oxidative degradation, requirement of special storage conditions and limited penetration ability to the skin [6] (Table 5). So that, the niosomes produced by nonionic surfactants such as polyoxyethylene alkyl ethers or esters and cholesterol have been developed by researchers in the cosmetic industry (L'Oreal) in $1975[104,106]$. These vesicles that resemble liposomes in their structures are between 100 and $2000 \mathrm{~nm}$ in size (Figure 3). They have higher encapsulation efficiency, improved chemical stability, enhanced penetration 
and lower cost compared with liposomes [106] (Table 5). The transfersomes that are another member of vesicular systems were designed in 1992 by Cevc and Blume [107]. These vesicles are comprised phospholipids and edge activators (such as sodium cholate and sodium deoxycholate) and enhance the skin permeation [108,109] (Figure 3). The transfersomes have flexible and deformable characteristics due to edge activators in their composition. Thus, these vesicles are also called as deformable or flexible liposomes [6]. The ethosomes are first reported in 2000 by Touitou et al. [110] and contain ethanol (up to 45\%; instead of cholesterol) used as constituent in ethosomes, unlikely conventional liposomes [111] (Figure 3). The ethosomes ensure enhancement of drug penetration into deeper layers of the skin, due to their soft and malleable characteristics because of high ethanol concentration [112] (Table 5).

\section{The potential penetration enhancement mechanisms of vesicular nanocarriers}

The most of the studies have explained that classical liposomes have not reached into viable skin layers and, only accumulated on the surface of the stratum corneum due to their large size and deficiency of elasticity (Table 4). Kwon et al. [113] examined the cellular uptake of liposomes conjugated with the cationic arginine-rich CPP. CLSM images have shown that Rhodamin B loaded in hydrophilic core of CPP-conjugated liposomes reached into viable skin layers, whereas FITC in the lipid bilayer of the liposomes remained in the upper epidermis, due to perturbation of phospholipids in the intercellular lipid lamellae of the stratum corneum. Also, the liposomes localized into hair follicles and acted as a drug reservoir [114,115] (Table 4). Li et al. [56] proved that the liposomes localized in flank organs (sebaceous glands, hair follicles and melanocytes clumps).

The liposomal drug delivery into the skin might be affected by their characteristic properties such as size, surface charge and lamellarity of vesicles, lipid composition and type of lipid [54,116,117]. Nohynek et al. [104] discussed the effect of vesicle size on skin penetration and clarified that nanosized of liposomes did not affect skin penetration. We have also assumed that the localization of liposomes in hair follicles might be affected by their size, thereby drug concentration in hair follicles. One of the earlier studies reported that negatively charged vesicles exhibited higher drug flux than positively charged vesicles [118]. Conversely, positively charged liposomes caused similar drug release to the negatively charged ones in another study [119]. Some researchers also showed that drug loaded into the liquid-state liposomes had greater penetration ability into the skin in comparison with the gel-state liposomes [120] due to the interaction of the stratum corneum lipids and liposomal lipids. The liposomes composed of saturated phospholipids with a phase-transition temperature $\left(\mathrm{T}_{\mathrm{m}}\right)$ above the temperature of the skin $\left(32^{\circ} \mathrm{C}\right)$ are in a rigid gel form when applied to the skin. Then, intercellular lipid deposition occurs among the stratum corneum lipids, decreases fluidity of the stratum corneum and gel-state liposomes remain in upper epidermis. In contrast to that the liposomes prepared with unsaturated phospholipids are in liquid form at $\mathrm{T}_{\mathrm{m}}$ below $32^{\circ} \mathrm{C}$, enhance the fluidity of the stratum corneum lipids due to perturbation of the lipid organization of the stratum corneum and, then the drug molecules loaded into the liposomes penetrate into viable epidermis.

Some researchers have claimed that the niosomes have enabled only cutaneous penetration of the drug, as the others have argued the possibility of transdermal drug delivery via niosomes [121-125]. However, it clarified that the penetration of niosomes depends on their characteristics such as composition, lamellarity, elasticity, surface charge and size $[126,127]$. Also, some researchers reported that transfollicular pathway is an active penetration mechanism for liquid-state niosomes [128], penetration enhancer containing niosomes [63] and the cationic niosomes [129]. In recent years, several strategies have developed to enhance drug transport via niosomes in the skin [61,62] (Table 4). One of those approaches is the addition of solubilizers into the niosomes, which act as penetration enhancer. Confocal laser scanning images displayed that fluorescence in PEG400-niosomes and Labrasol association to the diolein-niosomes reached into depth of $120 \mu \mathrm{m}$ and skin appendages, respectively [62,63]. The other strategy is incorporation of the niosomes into a gel matrix that provides improvement in the mechanical stability and membrane integrity of the niosomes $[124,130]$. Thus, increased drug penetration might be provided improving their stabilities and increasing their retention in the skin.

The enhancement of drug penetration or permeability via the niosomes is explained by three possible mechanisms: adsorption and fusion of the niosomes into the stratum corneum, then affecting intercellular lipids in the stratum corneum and improving skin penetration/permeation of the drug; acting of the niosomes as penetration enhancers due to the presence of surfactants in their structures and diffusing into the stratum corneum, resulted in impaired barrier function and improved penetration/permeation of the drug; localization of the niosomes into the hair follicles and release of drug from the niosomes $[121,123,130]$ (Table 4).

The transfersomes can penetrate sufficiently through skin pores due to their deformable characteristics [131]. Cevc [132] suggested that hydrophilic elastic vesicles squeezed into the narrow intercellular paths in the stratum 
corneum, consisted of microchannels formed of water-rich regions surrounded by polar lipids under the influence of the transepidermal water-activity gradient, resulting in the widening of the intercellular pathways up to 20 $30 \mathrm{~nm}$. Then, the deformable transfersomes moved into skin layers through the microchannels of skin along the transdermal hydration gradient responding to external stresses within the intercellular lipid packing of the stratum corneum. After passage, the transfersomes distributed between the corneocytes via intercellular paths, and localized in the subcutaneous tissue [67,132] (Table 4). These researchers have asserted that transfersomes penetrated through the skin barrier by this mechanism, reached into the subcutaneous tissue such as muscle and eliminate by the liver. Also, they indicated that the vesicle size did not change after barrier passage though the shape and volume adaptation transiently changed [132]. Therefore, the transfersomes are proposed as superior carriers over the conventional lipid vesicles for the enhancement of drug permeation and vesicle-skin interactions [132,133]. Also, Duangjit et al. [134] suggested that meloxicam-loaded transfersomes penetrated to the skin by disruption and fluidization of the stratum corneum lipids (act as penetration enhancers).

Bahia et al. [135] established that calcein-encapsulated deformable vesicles decreased the transdermal flux of the hydrophilic marker compared with its control solutions. They stated that deformable vesicles have acted as a sustained release system for hydrophilic drugs into the viable skin tissues. Cosco et al. [68] also demonstrated that transfersomes reached into deeper strata of the skin. Similarly, Oh et al. [67] reported that 5-aminolevulinic acid loaded into the cationic ultradeformable liposomes selectively localized in the epidermis. Ascenso et al. [66] and Perez et al. [65] also indicated that the drug in the transfersomes accumulated in the stratum corneum and viable epidermis.

The exact mechanism of skin permeation and penetration of the ethosomes is still not clear. However, phospholipids and high concentration of ethanol in the ethosomes have created a synergistic mechanism for drug penetration into deeper layers of the skin [136]. Ethanol increases the lipid fluidity both of the ethosomal and skin lipid bilayers and decreases the density of the highly ordered lipids of the stratum corneum. The soft flexible ethosomes perturbed intercellular lipid layers of the stratum corneum penetrate into the deeper skin layers. The drug diffuses into deeper skin layers or systemic circulation after fusion of the vesicle with the lipid contents of the stratum corneum [137] (Table 4).

The enhanced permeation and deposition of drug via the ethosomes into the epidermis are also available in the literature [69-71] (Table 4). Recently, improved stability and elasticity of the transethosomes compared with the other vesicles have intensively attracted attention [138]. Transethosomes with high concentration of ethanol (up to 30\%) and an edge activator are in irregular spherical shape and have higher values of elasticity and skin permeation/penetration characteristics than ethosomes and transfersomes [69]. The transethosomes increased deposition of voriconazole in the dermis/epidermis as well as dramatically enhanced the skin permeation of drug, in comparison with deformable liposomes and conventional liposomes [139].

\section{Conclusion \& future perspective}

The human skin, especially the stratum corneum, is an impermeable barrier for drug molecules as well as dust, bacteria, chemical agents. The conventional formulations exhibit insufficient drug penetration into viable skin layers due to this barrier. In recent years, the nanocarriers have considered as an alternative strategy to improve delivery and targeting of the drugs into the deeper skin layers. In particular, topical nanocarriers have attracted attention due to their potential for targeting of drugs into desired skin regions, drug release in a controlled manner and reducing side effects of drugs.

The penetration ability of nanocarriers through the stratum corneum barrier could be variable by the effect of various factors including the skin condition: intact, impaired and so on; the formulation characteristics: type of carrier, the presence of excipients such as penetration enhancers; the physicochemical properties of nanocarrier: size, surface charge, flexibility and so on; the skin model: human, porcine, pig, mouse, rat; the environmental and experimental conditions: time, temperature, occlusion, mechanical effects such as massage, flexing. It is generally supposed that the intact penetration of polymeric nanoparticles across the stratum corneum is negligible, they localize in the stratum corneum surface, furrows and hair follicles. Thus, the polymeric nanocarriers could be proposed as appropriate carriers to enable sustained and localized drug release onto the skin surface. Similarly, SLNs and NLCs are also considered as ideal carriers for epidermal and follicular targeting of drugs in the dermal delivery. The niosomes, transfersomes, ethosomes and transethosomes might intactly exceed the stratum corneum barrier via fusion mechanism. Therefore, they might be an alternative carrier for topical and transdermal drug administration. 
In conclusion, several studies have shown that topical nanocarriers have some superiorities for the treatment of dermatological diseases. Topical drug delivery via nanocarriers into deeper skin layers and hair follicles might be a promising strategy for the treatment of dermatological diseases, but it is crucial to understand the potential enhancement mechanisms of these novel carriers and further clinical, toxicological and pharmaceutical studies (such as release kinetics) are required.

\section{Financial \& competing interests disclosure}

The authors have no relevant affiliations or financial involvement with any organization or entity with a financial interest in or financial conflict with the subject matter or materials discussed in the manuscript. This includes employment, consultancies, honoraria, stock ownership or options, expert testimony, grants or patents received or pending, or royalties.

No writing assistance was utilized in the production of this manuscript.

Executive summary

Skin structure \& penetration pathways

- The skin has a unique barrier function due to the stratum corneum that is the uppermost layer of the skin.

- The stratum corneum is a rate-limiting barrier for penetration of large (>500 Da) and highly lipophilic molecules.

- The potential penetration pathways of drug molecules across the stratum corneum are intercellular, transcellular and/or transappendageal routes including hair follicles, sweat glands and sebaceous glands.

Nanocarrier-based drug delivery systems for dermal delivery

- Nanocarriers-based dermal-delivery systems have superiorities to improve cutaneous drug delivery and target the drugs into pilosebaceous units in comparison with the conventional dosage forms.

- Penetration of nanocarriers through the intact skin could be limited. However, nanosized delivery systems might be a good alternative for the treatment of dermatological diseases affecting skin and hair follicles.

Polymeric nanocarriers

- Polymeric nanocarriers have some advantages such as drug release in a controlled and sustained manner, plenty of preparation methods, despite the lack of toxicological assessment in the literature.

- The intact penetration of nanoparticles across the stratum corneum is negligible. They have localized in the stratum corneum surface, furrows and hair follicles. Then, the drug is released by passive diffusion from the polymeric nanoparticles in a sustained manner into viable skin layers.

- The polymeric nanoparticles could be appropriate carriers, which enable a sustained and localized drug release on the skin surface and hair follicles as a drug reservoir.

Lipid-based nanocarriers

- Lipid-based nanocarriers have less toxicity due to their biocompatible and biodegradable components in comparison with polymeric nanoparticles.

- Solid-lipid nanoparticles and nanostructured lipid carriers are proposed to be appropriate carriers for epidermal and follicular targeting of drugs in the dermal delivery.

- Liposomes remain on the stratum corneum surface because of their deficiency of flexibility and greater size while niosomes, transfersomes, ethosomes and transethosomes might intactly exceed the stratum corneum barrier via fusion.

Conclusion \& future perspective

- Polymeric nanocarriers, lipid nanocarriers (solid-lipid nanoparticles and nanostructured lipid carriers) and liposomes could be alternative carriers for treatment of skin diseases affecting epidermis and hair follicles.

- The niosomes, transfersomes, ethosomes and transethosomes enhance drug penetration up to deeper skin layers such as dermis.

- Nanocarriers for dermal delivery are promising systems, but further clinical and toxicological studies are still required for their commercialization and use in the treatment.

\section{References}

Papers of special note have been highlighted as: $\bullet$ of interest; $\bullet \bullet$ of considerable interest

1 Trommer H, Neubert RH. Overcoming the stratum corneum: the modulation of skin penetration. A review. Skin Pharmacol. Physiol. 19(2), 106-121 (2006).

2 Raza K, Singh B, Singal P, Wadhwa S, Katare OP. Systematically optimized biocompatible isotretinoin-loaded solid lipid nanoparticles (SLNs) for topical treatment of acne. Colloids Surf. B: Biointerfaces 105, 67-74 (2013).

3 Barua S, Mitragotri S. Challenges associated with penetration of nanoparticles across cell and tissue barriers: a review of current status and future prospects. Nano Today 9(2), 223-243 (2014). 
4 Gupta M, Agrawal U, Vyas SP. Nanocarrier-based topical drug delivery for the treatment of skin diseases. Expert Opin. Drug Deliv. 9(7), 783-804 (2012).

5 Raphael AP, Garrastazu G, Sonvico F, Prow TW. Formulation design for topical drug and nanoparticle treatment of skin disease. Ther. Deliv. 6(2), 197-216 (2015).

6 Roberts MS, Mohammed Y, Pastore MN et al. Topical and cutaneous delivery using nanosystems. J. Control. Rel. 247, 86-105 (2017).

7 Güngör S, Kahraman E, Özsoy Y. Polymeric micelles for cutaneous drug delivery. In: Nano Based Drug Delivery. Naik J (Ed.). IAPC Publishing, Zagreb, Croatia, 369-384 (2015).

8 Dragicevic-Curic N, Maibach HI. Percutaneous Penetration Enhancer Chemical Methods in Penetration Enhancement. Springer-Verlag, Berlin, Heidelberg, Germany (2015).

9 Ellias PM. Epidermal barrier function: intercellular lamellar lipid structures, origin composition and methabolism. J. Control. Rel. 5 , 199-208 (1991).

10 Menon GK. New insights into skin structure: scratching the surface. Adv. Drug Deliv. Rev. 54, S3-S17 (2002).

11 Hadgraft J, Lane ME. Skin: the ultimate interface. Phys. Chem. 13(12), 5215-5222 (2011).

12 Roberts MS, Cross ES, Pellet MA. Skin transport. In: Dermatological and Transdermal Formulations. Walters KA (Ed.). ABD, Marcel Dekker, Germany, 89-195 (2002).

13 Fang CL, Aljuffali IA, Li YC, Fang JY. Delivery and targeting of nanoparticles into hair follicles. Ther. Deliv. 5(9), $991-1006$ (2014).

14 Alvarez-Roman R, Naik A, Kalia YN, Guy RH, Fessi H. Skin penetration and distribution of polymeric nanoparticles. J. Control. Release 99(1), 53-62 (2004).

15 Bachhav YG, Mondon K, Kalia YN, Gurny R, Moller M. Novel micelle formulations to increase cutaneous bioavailability of azole antifungals. J. Control. Release 153(2), 126-132 (2011).

16 Venuganti VV, Perumal OP. Poly(amidoamine) dendrimers as skin penetration enhancers: influence of charge, generation, and concentration. J. Pharm. Sci. 98(7), 2345-2356 (2009).

17 Pardeike J, Hommoss A, Muller RH. Lipid nanoparticles (SLN, NLC) in cosmetic and pharmaceutical dermal products. Int. J. Pharm. 366(1-2), 170-184 (2009).

18 Unnithan AR, Sasikala AR, Murugesan P et al. Electrospun polyurethane-dextran nanofiber mats loaded with estradiol for post-menopausal wound dressing. Int. J. Biol. Macromol. 77, 1-8 (2015).

19 Abu Samah NH, Heard CM. The effects of topically applied poly NIPAM-based nanogels and their monomers on skin cyclooxygenase expression, ex vivo. Nanotoxicology 8(1), 100-106 (2014).

20 Zhang Z, Tsai PC, Ramezanli T, Michniak-Kohn BB. Polymeric nanoparticles-based topical delivery systems for the treatment of dermatological diseases. Wiley Interdiscip. Rev. Nanomed. Nanobiotechnol. 5(3), 205-218 (2013).

21 Venuganti VV, Saraswathy M, Dwivedi C, Kaushik RS, Perumal OP. Topical gene silencing by iontophoretic delivery of an antisense oligonucleotide-dendrimer nanocomplex: the proof of concept in a skin cancer mouse model. Nanoscale 7(9), 3903-3914 (2015).

22 Saydam M, Cheng WP, Palmer N et al. Nano-sized Soluplus ${ }^{\circledR}$ polymeric micelles enhance the induction of tetanus toxin neutralising antibody response following transcutaneous immunisation with tetanus toxoid. Vaccine 35(18), 2489-2495 (2017).

23 Campbell CS, Contreras-Rojas LR, Delgado-Charro MB, Guy RH. Objective assessment of nanoparticle disposition in mammalian skin after topical exposure. J. Control. Release 162(1), 201-207 (2012).

24 Wu X, Biatry B, Cazeneuve C, Guy RH. Drug delivery to the skin from sub-micron polymeric particle formulations: influence of particle size and polymer hydrophobicity. Pharm. Res. 26(8), 1995-2001 (2009).

-• Reported that polymeric nanocarriers do not penetrate into deeper skin layers, only remain superficial layers of the stratum corneum.

25 Vogt A, Combadiere B, Hadam S et al. $40 \mathrm{~nm}$, but not 750 or 1,500 nm, nanoparticles enter epidermal CD1a+ cells after transcutaneous application on human skin. J. Invest. Dermatol. 126(6), 1316-1322 (2006).

26 Lademann J, Richter H, Teichmann A et al. Nanoparticles an efficient carrier for drug delivery into the hair follicles. Eur. J. Pharm. Biopharm. 66(2), 159-164 (2007).

27 Mathes C, Melero A, Conrad P et al. Nanocarriers for optimizing the balance between interfollicular permeation and follicular uptake of topically applied clobetasol to minimize adverse effects. J. Control. Rel. 223, 207-214 (2016).

28 Morgen M, Lu GW, Du D et al. Targeted delivery of a poorly water-soluble compound to hair follicles using polymeric nanoparticle suspensions. Int. J. Pharm. 416(1), 314-322 (2011).

29 Abdel-Mottaleb MM, Moulari B, Beduneau A, Pellequer Y, Lamprecht A. Nanoparticles enhance therapeutic outcome in inflamed skin therapy. Eur. J. Pharm. Biopharm. 82(1), 151-157 (2012).

30 Arayachukeat S, Wanichwecharungruang SP, Tree-Udom T. Retinyl acetate-loaded nanoparticles: dermal penetration and release of the retinyl acetate. Int. J. Pharm. 404(1-2), 281-288 (2011). 
31 Badihi A, Debotton N, Frusic-Zlotkin M, Soroka Y, Neuman R, Benita S. Enhanced cutaneous bioavailability of dehydroepiandrosterone mediated by nano-encapsulation. J. Control. Rel. 189, 65-71 (2014).

32 Teixeira Z, Zanchetta B, Melo BA et al. Retinyl palmitate flexible polymeric nanocapsules: characterization and permeation studies. Colloids Surf. B Biointerfaces 81(1), 374-380 (2010).

33 Batheja P, Sheihet L, Kohn J, Singer AJ, Michniak-Kohn B. Topical drug delivery by a polymeric nanosphere gel: formulation optimization and in vitro and in vivo skin distribution studies. J. Control. Rel. 149(2), 159-167 (2011).

34 Try C, Moulari B, Beduneau A et al. Size dependent skin penetration of nanoparticles in murine and porcine dermatitis models. Eur. J. Pharm. Biopharm. 100, 101-108 (2016).

35 Shah PP, Desai PR, Singh M. Effect of oleic acid modified polymeric bilayered nanoparticles on percutaneous delivery of spantide II and ketoprofen. J. Control. Rel. 158(2), 336-345 (2012).

36 Lapteva M, Santer V, Mondon K et al. Targeted cutaneous delivery of ciclosporin A using micellar nanocarriers and the possible role of inter-cluster regions as molecular transport pathways. J. Control. Rel. 196, 9-18 (2014).

37 Lapteva M, Mondon K, Moller M, Gurny R, Kalia YN. Polymeric micelle nanocarriers for the cutaneous delivery of tacrolimus: a targeted approach for the treatment of psoriasis. Mol. Pharm. 11(9), 2989-3001 (2014).

38 Lapteva M, Moller M, Gurny R, Kalia YN. Self-assembled polymeric nanocarriers for the targeted delivery of retinoic acid to the hair follicle. Nanoscale 7(44), 18651-18662 (2015).

- Indicated that the polymeric micelles enabled an increased and targeted delivery of drug to the pilosebaceous units.

39 Borowska K, Wolowiec S, Rubaj A, Glowniak K, Sieniawska E, Radej S. Effect of polyamidoamine dendrimer G3 and G4 on skin permeation of 8-methoxypsoralene in vivo study. Int. J. Pharm. 426(1-2), 280-283 (2012).

40 Venuganti VV, Sahdev P, Hildreth M, Guan X, Perumal O. Structure-skin permeability relationship of dendrimers. Pharm. Res. 28(9), 2246-2260 (2011).

41 Mutalik S, Shetty PK, Kumar A, Kalra R, Parekh HS. Enhancement in deposition and permeation of 5-fluorouracil through human epidermis assisted by peptide dendrimers. Drug Deliv. 21(1), 44-54 (2014).

42 Agrawal U, Mehra NK, Gupta U, Jain NK. Hyperbranched dendritic nano-carriers for topical delivery of dithranol. J. Drug Target. 21(5), 497-506 (2013).

43 Yang Y, Sunoqrot S, Stowell C et al. Effect of size, surface charge, and hydrophobicity of poly(amidoamine) dendrimers on their skin penetration. Biomacromolecules 13(7), 2154-2162 (2012).

44 Jensen LB, Petersson K, Nielsen HM. In vitro penetration properties of solid lipid nanoparticles in intact and barrier-impaired skin. Eur. J. Pharm. Biopharm. 79(1), 68-75 (2011).

45 Iannuccelli V, Coppi G, Romagnoli M, Sergi S, Leo E. In vivo detection of lipid-based nano- and microparticles in the outermost human stratum corneum by EDX analysis. Int. J. Pharm. 447(1-2), 204-212 (2013).

46 Keshri L, Pathak K. Development of thermodynamically stable nanostructured lipid carrier system using central composite design for zero order permeation of econazole nitrate through epidermis. Pharm. Dev. Technol. 18(3), 634-644 (2013).

47 Montenegro L, Sinico C, Castangia I, Carbone C, Puglisi G. Idebenone-loaded solid lipid nanoparticles for drug delivery to the skin: in vitro evaluation. Int. J. Pharm. 434(1-2), 169-174 (2012).

48 Jain AK, Jain A, Garg NK et al. Adapalene loaded solid lipid nanoparticles gel: an effective approach for acne treatment. Colloids Surf. B Biointerfaces 121, 222-229 (2014).

49 Chen S, Liu W, Wan J et al. Preparation of Coenzyme Q10 nanostructured lipid carriers for epidermal targeting with high-pressure microfluidics technique. Drug Dev. Ind. Pharm. 39(1), 20-28 (2013).

50 Wosicka-Frackowiak H, Cal K, Stefanowska J et al. Roxithromycin-loaded lipid nanoparticles for follicular targeting. Int. J. Pharm. 495(2), 807-815 (2015)

51 Aljuffali IA, Sung CT, Shen FM, Huang CT, Fang JY. Squarticles as a lipid nanocarrier for delivering diphencyprone and minoxidil to hair follicles and human dermal papilla cells. AAPS J. 16(1), 140-150 (2014).

52 Andrade LM, de Fatima Reis C, Maione-Silva L et al. Impact of lipid dynamic behavior on physical stability, in vitro release and skin permeation of genistein-loaded lipid nanoparticles. Eur. J. Pharm. Biopharm. 88(1), 40-47 (2014).

53 Charoenputtakun P, Pamornpathomkul B, Opanasopit P, Rojanarata T, Ngawhirunpat T. Terpene composited lipid nanoparticles for enhanced dermal delivery of all-trans-retinoic acids. Biol. Pharm. Bull. 37, 1139-1148 (2014).

54 Knudsen NO, Jorgensen L, Hansen J, Vermehren C, Frokjaer S, Foged C. Targeting of liposome-associated calcipotriol to the skin: effect of liposomal membrane fluidity and skin barrier integrity. Int. J. Pharm. 416(2), 478-485 (2011).

55 Campani V, Marchese D, Pitaro MT, Pitaro M, Grieco P, De Rosa G. Development of a liposome-based formulation for vitamin K1 nebulization on the skin. Int. J. Nanomedicine 9, 1823-1832 (2014).

$56 \mathrm{Li} \mathrm{L}$, Tang L, Baranov E et al. Selective induction of apoptosis in the hamster flank sebaceous gland organ by a topical liposome 5-alpha-reductase inhibitor: a treatment strategy for acne. J. Dermatol. 37(2), 156-162 (2010). 
57 Srisuk P, Thongnopnua P, Raktanonchai U, Kanokpanont S. Physico-chemical characteristics of methotrexate-entrapped oleic acid-containing deformable liposomes for in vitro transepidermal delivery targeting psoriasis treatment. Int. J. Pharm. 427(2), 426-434 (2012).

58 Manconi M, Sinico C, Caddeo C, Vila AO, Valenti D, Fadda AM. Penetration enhancer containing vesicles as carriers for dermal delivery of tretinoin. Int. J. Pharm. 412(1-2), 37-46 (2011).

59 Mura S, Manconi M, Sinico C, Valenti D, Fadda AM. Penetration enhancer-containing vesicles (PEVs) as carriers for cutaneous delivery of minoxidil. Int. J. Pharm. 380(1-2), 72-79 (2009).

60 Manconi M, Caddeo C, Sinico C et al. Ex vivo skin delivery of diclofenac by transcutol containing liposomes and suggested mechanism of vesicle-skin interaction. Eur. J. Pharm. Biopharm. 78(1), 27-35 (2011).

61 Pando D, Matos M, Gutierrez G, Pazos C. Formulation of resveratrol entrapped niosomes for topical use. Colloids Surf. B Biointerfaces 128, 398-404 (2015).

62 Junyaprasert VB, Singhsa P, Suksiriworapong J, Chantasart D. Physicochemical properties and skin permeation of Span 60/Tween 60 niosomes of ellagic acid. Int. J. Pharm. 423(2), 303-311 (2012).

63 Manca ML, Manconi M, Nacher A et al. Development of novel diolein-niosomes for cutaneous delivery of tretinoin: influence of formulation and in vitro assessment. Int. J. Pharm. 477(1-2), 176-186 (2014).

64 Subongkot T, Wonglertnirant N, Songprakhon P, Rojanarata T, Opanasopit P, Ngawhirunpat T. Visualization of ultradeformable liposomes penetration pathways and their skin interaction by confocal laser scanning microscopy. Int. J. Pharm. 441(1-2), 151-161 (2013).

65 Perez AP, Altube MJ, Schilrreff P et al. Topical amphotericin B in ultradeformable liposomes: formulation, skin penetration study, antifungal and antileishmanial activity in vitro. Colloids Surf. B Biointerfaces 139, 190-198 (2016).

66 Ascenso A, Salgado A, Euleterio C et al. In vitro and in vivo topical delivery studies of tretinoin-loaded ultradeformable vesicles. Eur. J. Pharm. Biopharm. 88(1), 48-55 (2014).

67 Oh EK, Jin SE, Kim JK, Park JS, Park Y, Kim CK. Retained topical delivery of 5-aminolevulinic acid using cationic ultradeformable liposomes for photodynamic therapy. Eur. J. Pharm. Sci. 44(1-2), 149-157 (2011).

68 Cosco D, Paolino D, Maiuolo J et al. Ultradeformable liposomes as multidrug carrier of resveratrol and 5-fluorouracil for their topical delivery. Int. J. Pharm. 489(1-2), 1-10 (2015).

69 Ascenso A, Raposo S, Batista C et al. Development, characterization, and skin delivery studies of related ultradeformable vesicles: transfersomes, ethosomes, and transethosomes. Int. J. Nanomedicine 10, 5837-5851 (2015).

70 Akhtar N, Pathak K. Cavamax W7 composite ethosomal gel of clotrimazole for improved topical delivery: development and comparison with ethosomal gel. AAPS PharmSciTech. 13(1), 344-355 (2012).

71 Verma P, Pathak K. Nanosized ethanolic vesicles loaded with econazole nitrate for the treatment of deep fungal infections through topical gel formulation. Nanomedicine 8(4), 489-496 (2012).

72 Uchechi O, Ogbonna JDN, Attama AA. Chapter 6. Nanoparticles for dermal and transdermal drug delivery. In: Application of Nanotechnology in Drug Delivery. Sezer AD (Ed.). InTech, Zagreb, Croatia (2014).

73 Couvreur P, Dubernet C, Puisieux F. Controlled drug delivery with nanoparticles: current possibilities and future trends. Eur. J. Pharm. Biopharm. 41, 2-13 (1995).

74 Vauthier C, Couvreur P. Development of nanoparticles made of polysaccharides as novel drug carrier systems. In: Handbook of Pharmaceutical Controlled Release Technology. Wise DL (Ed.). Marcel Dekker, NY, USA, 13-429 (2000).

75 Panyam J, Labhasetwar V. Biodegradable nanoparticles for drug and gene delivery to cells and tissue. Adv. Drug Deliv. Rev. 55, 329-347 (2003).

76 Torchilin VP. Block copolymer micelles as a solution for drug delivery problems. Expert Opin. Ther. Pat. 15, 63-75 (2005).

77 Torchilin VP. Structure and design of polymeric surfactant-based drug delivery systems. J. Control. Rel. 73, 137-172 (2001).

78 Lee SC, Huh KM, Ooya T, Park K. Hydrotropic polymer micelles for cancer therapeutics. In: Nanotechnology for Cancer Therapeutics. Amiji M (Ed.). CRC Press, USA, 385-408 (2007).

79 Cha EJ, Kim JE, Ahn CH. Stabilized polymeric micelles by electrostatic interactions for drug delivery system. Eur. J. Pharm. Sci. 38(4), 341-346 (2009).

80 Dianzani C, Zara GP, Maina G et al. Drug delivery nanoparticles in skin cancers. Biomed Res. Int. 895986 (2014).

81 Matos BN, Reis TA, Gratieri T, Gelfuso GM. Chitosan nanoparticles for targeting and sustaining minoxidil sulphate delivery to hair follicles. Int. J. Biol. Macromol. 75, 225-229 (2015).

82 Abdel-Mottaleb MM, Neumann D, Lamprecht A. Lipid nanocapsules for dermal application: a comparative study of lipid-based versus polymer-based nanocarriers. Eur. J. Pharm. Biopharm. 79(1), 36-42 (2011).

83 Laredj-Bourezg F, Bolzinger MA, Pelletier J et al. Skin delivery by block copolymer nanoparticles (block copolymer micelles). Int. J. Pharm. 496(2), 1034-1046 (2015). 
84 Kahraman E, Ozhan G, Ozsoy Y, Gungor S. Polymeric micellar nanocarriers of benzoyl peroxide as potential follicular targeting approach for acne treatment. Colloids Surf. B Biointerfaces 146, 692-699 (2016).

85 Smejkalová D, Muthny T, Nesporova K et al. Hyaluronan polymeric micelles for topical drug delivery. Carbohydr. Polym. 156, 86-96 (2017).

86 Yang J, Hu J, He B, Cheng Y. Transdermal delivery of therapeutic agents using dendrimers (US20140018435A1): a patent evaluation. Exp. Opin. Ther. Patents 25, 1209-1214 (2015).

87 Sun M, Fan A, Wang Z, Zhao Y. Dendrimer-mediated drug delivery to the skin. Soft Matter 8, 4301-4305 (2012).

88 Meidan VM, Walmsley AD, Docker MF, Irwin WJ. Ultrasound-enhanced diffusion into coupling gel during phonophoresis of 5-fluorouracil. Int. J. Pharm. 185, 205-213 (1999).

89 Merino V, Lopez A, Kalia YN, Guy RH. Electrorepulsion versus electroosmosis: effect of $\mathrm{pH}$ on the iontophoretic flux of 5-fluorouracil. Pharm. Res. 16, 758-761 (1999).

90 Lee WR, Shen SC, Wang KH, Hu CH, Fang JY. The effect of laser treatment on skin to enhance and control transdermal delivery of 5-fluorouracil. J. Pharm. Sci. 91, 1613-1626 (2002).

91 Beall HD, Sloan KB. Topical delivery of 5-fluorouracil (5-FU) by 1, 3-bisalkylcarbonyl- 5-FU prodrugs. Int. J. Pharm. 231, 43-49 (2002).

92 Mehnert W, Mader K. Solid lipid nanoparticles: production, characterization and applications. Adv. Drug Deliv. Rev. 47, 165-196 (2001).

93 Wissing SA, Müller RH. Cosmetic applications for solid lipid nanoparticles (SLN). Int. J. Pharm. 254, 65-68 (2003).

94 Gokce EH, Korkmaz E, Tuncay-Tanriverdi S et al. A comparative evaluation of coenzyme Q10-loaded liposomes and solid lipid nanoparticles as dermal antioxidant carriers. Int. J. Nanomedicine 7, 5109-5117 (2012).

95 Javadzadeh Y, Bahari LA. Therapeutic nanostructures for dermal and transdermal drug delivery. In: Nano- and Micro-scale Drug Delivery Systems: Design and Fabrication. Grumezescu A (Ed.). Elsevier, USA, 131-142 (2017).

96 Lauterbach A, Müller-Goymann CC. Comparison of rheological properties, follicular penetration, drug release, and permeation behavior of a novel topical drug delivery system and a conventional cream. Eur. J. Pharm. Biopharm. 88, 614-624 (2014).

97 Küchler S, Radowski MR, Blaschke T et al. Nanoparticles for skin penetration enhancement: a comparison of a dendritic core-multishell-nanotransporter and solid lipid nanoparticles. Eur. J. Pharm. Biopharm. 71(2), 243-250 (2009).

98 Khurana S, Bedi PM, Jain NK. Preparation and evaluation of solid lipid nanoparticles based nanogel for dermal delivery of meloxicam. Chem. Phys. Lipids 175-176, 65-72 (2013).

99 Zhang J, Smith E. Percutaneous permetion of betamethasone 17-valerate incorporated in lipid nanoparticles. J. Pharm. Sci. 100, 896-903 (2011).

100 Lauterbach A, Muller-Goymann CC. Applications and limitations of lipid nanoparticles in dermal and transdermal drug delivery via the follicular route. Eur. J. Pharm. Biopharm. 97(Pt A), 152-163 (2015).

101 Desai PR, Shah PP, Patlolla RR, Singh M. Dermal microdialysis technique to evaluate the trafficking of surface-modified lipid nanoparticles upon topical application. Pharm. Res. 29, 2587-2600 (2012).

102 Shah PP, Desai PR, Channer D, Singh M. Enhanced skin permeation using polyarginine modified nanostructured lipid carriers. J. Control. Rel. 161, 735-745 (2012).

103 Patlolla RR, Desai PR, Belay K, Singh MS. Translocation of cell penetrating peptide engrafted nanoparticles across skin layers. Biomaterials 31, 5598-5607 (2010).

104 Nohynek GJ, Dufour EK, Roberts MS. Nanotechnology, cosmetics and the skin: is there a health risk? Skin Pharmacol. Physiol. 21(3), 136-149 (2008).

105 Lohani A, Verma A, Joshi H, Yadav N, Karki N. Nanotechnology-based cosmeceuticals. ISRN Dermatol. 843687 (2014).

106 Kazi KM, Mandal AS, Kuotsu K. Niosome: a future of targeted drug delivery systems. J. Adv. Pharm. Tech. \& Res. 1(4), 374-380 (2010).

107 Cevc G, Blume G. Lipid vesicles penetrate into intact skin owing to the transdermal osmotic gradients and hydration force. Biochim. Biophys. Acta. 1104, 226-232 (1992).

108 Zhang Y, Ng W, Feng X, Cao F, Xu H. Lipid vesicular nanocarrier: quick encapsulation efficiency determination and transcutaneous application. Int. J. Pharm. 516(1-2), 225-230 (2017).

109 Avadhani KS, Manikkath J, Tiwari M et al. Skin delivery of epigallocatechin-3-gallate (EGCG) and hyaluronic acid loaded nano-transfersomes for antioxidant and anti-aging effects in UV radiation induced skin damage. Drug Deliv. 24(1), 61-74 (2017).

110 Touitou E, Dayan N, Bergelson L, Godin B, Eliaz M. Ethosomes - novel vesicular carriers for enhanced delivery: characterization and skin penetration properties. J. Control. Rel. 65, 403-418 (2000).

111 Mbah CC, Builders PF, Attama AA. Nanovesicular carriers as alternative drug delivery systems: ethosomes in focus. Exp. Opin. Drug Deliv. 11, 45-59 (2014).

112 Touitou E. Composition of applying active substances to or through the skin. US5716638 (1996). 
113 Kwon SS, Kim SY, Kong BJ et al. Cell penetrating peptide conjugated liposomes as transdermal delivery system of Polygonum aviculare L. extract. Int. J. Pharm. 483(1-2), 26-37 (2015).

114 Hoffman RM. Topical liposome targeting of dyes, melanins, genes, and proteins selectively to hair follicles. J. Drug Target. 5(2), 67-74 (1998).

115 El Maghraby GM, Barry BW, Williams AC. Liposomes and skin: from drug delivery to model membranes. Eur. J. Pharm. Sci. 34(4-5), 203-222 (2008).

116 Babu S, Fan C, Stepanskiy L, Uitto J, Papazoglou E. Effect of size at the nanoscale and bilayer rigidity on skin diffusion of liposomes. J. Biomed. Mater. Res. A 91(1), 140-148 (2009).

117 Shanmugam S, Song CK, Nagayya-Sriraman S et al. Physicochemical characterization and skin permeation of liposome formulations containing clindamycin phosphate. Arch. Pharm. Res. 32(7), 1067-1075 (2009).

118 Montenegro L, Panico AM, Ventimiglia A, Bonina FP. In vitro retinoic acid release and skin permeation from different liposome formulations. Int. J. Pharm. 133, 89-96 (1996).

119 Sinico C, Manconi M, Peppi M, Lai F, Valenti D, Fadda AM. Liposomes as carriers for dermal delivery of tretinoin: in vitro evaluation of drug permeation and vesicle-skin interaction. J. Control. Rel. 103(1), 123-136 (2005).

120 Perez-Cullell N, Coderch L, de la Maza A, Parra JL, Estelrich J. Influence of the fluidity of liposome compositions on percutaneous absorption. Drug Deliv. 7, 7-13 (2000).

121 Alsarra IA, Bosela AA, Ahmed SM, Mahrous GM. Proniosomes as a drug carrier for transdermal delivery of ketorolac. Eur. J. Pharm. Biopharm. 59(3), 485-490 (2005).

122 Paolino D, Cosco D, Muzzalupo R, Trapasso E, Picci N, Fresta M. Innovative bola-surfactant niosomes as topical delivery systems of 5-fluorouracil for the treatment of skin cancer. Int. J. Pharm. 353(1-2), 233-242 (2008).

123 Balakrishnan P, Shanmugam S, Lee WS et al. Formulation and in vitro assessment of minoxidil niosomes for enhanced skin delivery. Int. J. Pharm. 377(1-2), 1-8 (2009).

124 Tavano L, Gentile L, Oliviero Rossi C, Muzzalupo R. Novel gel-niosomes formulations as multicomponent systems for transdermal drug delivery. Colloids Surf. B Biointerfaces. 110, 281-288 (2013).

125 Zhang Y, Zhang $\mathrm{K}$, Wu Z et al. Evaluation of transdermal salidroside delivery using niosomes via in vitro cellular uptake. Int. J. Pharm. 478(1), 138-146 (2015).

126 Manconi M, Sinico C, Valenti D, Lai F, Fadda AM. Niosomes as carriers for tretinoin. III. A study into the in vitro cutaneous delivery of vesicle-incorporated tretinoin. Int. J. Pharm. 311(1-2), 11-19 (2006).

127 Choi MJ, Maibach HI. Liposomes and niosomes as topical drug delivery systems. Skin Pharmacol. Physiol. 18(5), 209-219 (2005).

128 Tabbakhian M, Tavakoli N, Jaafari MR, Daneshamouz S. Enhancement of follicular delivery of finasteride by liposomes and niosomes: 1. In vitro permeation and in vivo deposition studies using hamster flank and ear models. Int. J. Pharm. 323(1-2), 1-10 (2006).

129 Manosroi A, Ruksiriwanich W, Abe M, Manosroi W, Manosroi J. Transfollicular enhancement of gel containing cationic niosomes loaded with unsaturated fatty acids in rice (Oryza sativa) bran semi-purified fraction. Eur. J. Pharm. Biopharm. 81(2), 303-313 (2012).

130 Ioele G, Tavano L, De Luca M, Ragno G, Picci N, Muzzalupo R. Photostability and ex-vivo permeation studies on diclofenac in topical niosomal formulations. Int. J. Pharm. 494(1), 490-497 (2015).

131 Jain S, Jain P, Umamaheshwari RB, Jain NK. Transfersomes, a novel vesicular carrier for enhanced transdermal delivery: development, characterization, and performance evaluation. Drug Dev. Ind. Pharm. 29, 1013-1026 (2003).

132 Cevc G. Lipid vesicles and other colloids as drug carriers on the skin. Adv. Drug Deliv. Rev. 56(5), 675-711 (2004).

133 Dragicevic-Curic N, Gräfe S, Gitter B, Winter S, Fahr A. Surface charged temoporfin-loaded flexible vesicles: in vitro skin penetration studies and stability. Int. J. Pharm. 384, 100-108 (2010).

134 Duangit S, Opanasopit P, Rojanarata T, Ngawhirunpat T. Characterization and in vitro skin permeation of meloxicam-loaded liposomes versus transfersomes. J. Drug Deliv. 418316 (2011).

135 Bahia AP, Azevedo EG, Ferreira LA, Frezard F. New insights into the mode of action of ultradeformable vesicles using calcein as hydrophilic fluorescent marker. Eur. J. Pharm. Sci. 39(1-3), 90-96 (2010).

136 Touitou E, Godin B, Dayan N, Weiss C, Piliponsky A, Levi-Schaffer F. Intracellular delivery mediated by an ethosomal carrier. Biomaterials 22, 3053-3059 (2001).

137 Verma P, Pathak K. Therapeutic and cosmeceutical potential of ethosomes: an overview. J. Adv. Pharm. Technol. Res. 1(3), 274-282 (2010).

138 Garg V, Singh H, Bhatia A et al. Systematic development of transethosomal gel system of piroxicam: formulation optimization, in vitro evaluation, and ex vivo assessment. AAPS PharmSciTech. 18(1), 58-71 (2016).

139 Song YK, Hyun SY, Kim HT, Kim CK, Oh JM. Transdermal delivery of low molecular weight heparin loaded in flexible liposomes with bioavailability enhancement: comparison with ethosomes. J. Microencapsul. 28(3), 151-158 (2011). 



\title{
Integration of nano drug-delivery system with cancer immunotherapy
}

\author{
Takashi Nakamura ${ }^{1}$ \& Hideyoshi Harashima*,1 \\ ${ }^{1}$ Faculty of Pharmaceutical Sciences, Hokkaido University, Kita 12 Nishi 6, Kita-ku, Sapporo, Hokkaido 060-0812, Japan \\ * Author for correspondence: Tel.: +81 11706 3919; Fax: +81 11706 3734; harasima@pharm.hokudai.ac.jp
}

Immune checkpoint therapy represents a new, revolutionary type of cancer therapy, but emerging evidence indicates that only a minority of patients will benefit from it. The issue of how to improve and widen the clinical response is a pivotal issue, and combining other types of therapy with immune checkpoint inhibitors is currently under development. A nanotechnology-based drug-delivery system (nano DDS) could be an important contribution to the development of an effective combination therapy. In this document, we review recent findings in the field of tumor immunology, which provide a strategy for an efficient combination therapy, and discuss nano DDS that are associated with cancer immunotherapy and nano DDS strategies based on the immune status in tumor microenvironments.

First draft submitted: 29 June 2017; Accepted for publication: 4 September 2017; Published online: 24 October 2017

Keywords: adjuvant $\bullet$ cancer immunotherapy $\bullet$ drug-delivery system $\bullet$ immune checkpoint inhibitor $\bullet$ lipid nanoparticle • nanotechnology $\bullet$ nucleic acid $\bullet$ STING

Up until a few years ago, the use of immunotherapy in the field of cancer therapy was received with skepticism. The impregnable ranks have been surgery, radiation, chemotherapy and targeted therapy. However, the appearance of immune checkpoint inhibitors marked a turning point in cancer therapy. The success of immune checkpoint therapy has revolutionized the field of cancer therapy and immunotherapy has joined the impregnable ranks as a pillar. As of this writing, three types of immune checkpoint inhibitors have been approved; anti-CTLA-4, anti-PD-1, anti-PD ligand 1 (anti-PD-L1) as of August 2017 (Table 1). Anti-PD-1 is making particularly rapid progress, has widened such applications and achieved the first line application against non-small-cell lung cancer (NSCLC). However, positive responses were observed only in a minority of the treated patients and tumor types, and several resistance mechanisms have been identified. The emerging focus is on improving the response rate and overcoming resistance, and combination therapies are currently being evaluated in ongoing clinical trials. In combination therapies, anitPD-1 is a fundamental therapy and other cancer therapies such as radiation, chemotherapy, targeted therapy, tumor antigens, adjuvants, anti-CTLA-4, inhibitor, cell therapy, are combined with anti-PD-1. The efficacy of anti-PD1 is largely dependent on the immunogenicity of the tumor microenvironment (TME) [1]. Immunogenicity is basically defined as the ability of an antigen to prime either T or B cells [2]. T-cell infiltration and PD-L1 expression are observed in immunogenic TME, and anti-PD-1 monotherapy has shown durable clinical benefits $[1,3]$. Thus, in combination therapy, major efforts involve remodeling nonimmunogenic TME to immunogenic TME.

Nanotechnology-based drug-delivery systems (nano DDS) are an important contribution to the development of effective combination therapy. Nanoparticle formulations such as micelles, liposomes, lipid nanoparticles (LNPs), polyplexes and emulsions, are often used for assisting immunotherapy $[4,5]$. The leading stars in immunotherapeutic materials are tumor antigens (protein or peptide) and adjuvants, and they contribute to the remodeling to immunogenic TME. However, they are easily degraded and have a low affinity for immune cells, resulting in failure to induce effective immune responses. These cargos can be protected from degradation and delivered to target tissues, cells or organelles when they are loaded in nanoparticles. Nanoparticles have largely contributed to the promotion of antigen presentation, the activation of antigen-presenting cells (APCs), targeting dendritic cells (DCs), targeting lymph tissues, etc. Nano DDS therefore, represent a fundamental technology in cancer immunotherapy. Meanwhile, recent advances in tumor immunology require strategically combined immunotherapy based on the 


\begin{tabular}{|c|c|c|c|c|}
\hline Name & Company & Target & Approval & Indications \\
\hline $\begin{array}{l}\text { Ipilimumab } \\
\text { (Yervoy) }\end{array}$ & Bristol-Myers Squibb & CTLA-4 & 2011 & $\begin{array}{l}\checkmark \text { Unresectable or metastatic melanoma } \\
\checkmark \text { Adjuvant treatment of melanoma }\end{array}$ \\
\hline $\begin{array}{l}\text { Nivolumab } \\
\text { (Opdivo) }\end{array}$ & Bristol-Myers Squibb & PD-1 & 2014 & $\begin{array}{l}\checkmark \text { Unresectable or metastatic melanoma } \\
\checkmark \text { Metastatic non-small-cell lung cancer } \\
\checkmark \text { Renal cell carcinoma } \\
\checkmark \text { Classical Hodgkin lymphoma } \\
\checkmark \text { Squamous cell carcinoma of the head and neck } \\
\checkmark \text { Urothelial carcinoma } \\
\checkmark \text { MSI-H or mismatch repair } \\
\checkmark \text { Deficient (dMMR) metastatic colorectal cancer }\end{array}$ \\
\hline $\begin{array}{l}\text { Pembrolizumab } \\
\text { (Keytruda) }\end{array}$ & MSD & PD-1 & 2014 & $\begin{array}{l}\checkmark \text { Melanoma } \\
\checkmark \text { Non-small-cell lung cancer } \\
\checkmark \text { Head and neck cancer } \\
\checkmark \text { Classical Hodgkin lymphoma } \\
\checkmark \text { Urothelial carcinoma } \\
\checkmark \text { Microsatellite instability-high cancer }\end{array}$ \\
\hline $\begin{array}{l}\text { Atezolizumab } \\
\text { (Tecentriq) }\end{array}$ & $\begin{array}{l}\text { Roche } \\
\text { Genentech }\end{array}$ & PD-L1 & 2016 & $\begin{array}{l}\checkmark \text { Locally advanced or metastatic urothelial carcinoma } \\
\checkmark \text { Metastatic non-small-cell lung cancer }\end{array}$ \\
\hline $\begin{array}{l}\text { Avelumab } \\
\text { (Bavencio) }\end{array}$ & $\begin{array}{l}\text { Merck Serono } \\
\text { Pfizer }\end{array}$ & PD-L1 & 2017 & $\begin{array}{l}\checkmark \text { MCC } \\
\checkmark \text { Locally advanced or metastatic urothelial carcinoma }\end{array}$ \\
\hline $\begin{array}{l}\text { Durvalumab } \\
\text { (Imfinzi) }\end{array}$ & AstraZeneca & PD-L1 & 2017 & $\checkmark$ Locally advanced or metastatic urothelial carcinoma \\
\hline
\end{tabular}

immune status in TME. Thus, the development of nano DDS focused on the immune status in TME will be necessary. Nano DDS must be also revolutionized.

Herein, we review recent findings in the field of tumor immunology, which provides a strategy for efficient combination therapy, and discuss types of nano DDS that are associated with cancer immunotherapy and a nano DDS strategy based on the immune status in TME. Although, nano DDS is an important technique for strengthening combination immunotherapy, our intent was to emphasize that it is necessary to change the development strategy of nano DDS for cancer immunotherapy in order for it to efficiently enhance the combination immunotherapy tailored to the immune status of individuals in the near future.

\section{Spontaneous immune responses in tumor microenvironment}

In our body, the immune system protects us from cancer invasion. In the past, this type of protection was termed 'cancer immunosurveillance' [6]. However, the concept of cancer immunosurveillance has now expanded to 'cancer immunoediting', largely because of the much broader and complex interaction between tumors and immune systems. Cancer immunoediting consists of elimination, equilibrium and escape [7]. Cancer immunosurveillance represents the elimination phase. In the elimination phase, the recognition of transformed cells by immune systems (APCs, T cells, natural killer cells) is occurred, leading to the killing of transformed cells. If some tumor remains in the elimination phase, the process can then progress to the equilibrium phase. In the equilibrium phase, tumor cells persist but their growth is prevented by immune pressure. Genetic instability in tumor cells and immune selection of tumor cells then occurs. In the final phase, the escape phase, the balance between tumor cells and the immune system begin to tilt toward tumor growth as a consequence of immune inhibition or exhaustion, tumor cell mutation. The PD-1/PD-L1 pathway is one type of immune inhibition in the escape phase. As a result, cancer becomes clinically evident. The immune status in TME of cancer patients differs depending on the mechanism for the escape phase.

One of the most important features governing the success of immunotherapy is the presence of tumor-specific $\mathrm{T}$ cells in TME. To induce the infiltration of $\mathrm{T}$ cells, recognition of the tumor cells by the immune system, namely innate immune sensing, is the most proximal event. The initiation of innate immune sensing requires the recruitment of APCs into TME. Macrophages and DCs are classified as APCs. Both cells have heterogeneous subsets. Which APC initiates innate immune sensing in TME? It has been reported that a subset of DCs, driven by the transcription factor Batf3, are crucial for the induction of an antitumor response [8,9]. There are two major subsets of DCs, plasmacytoid DCs and conventional CD11 ${ }^{+}$DCs (cDCs) [10]. The subsets of cDCs are classified 


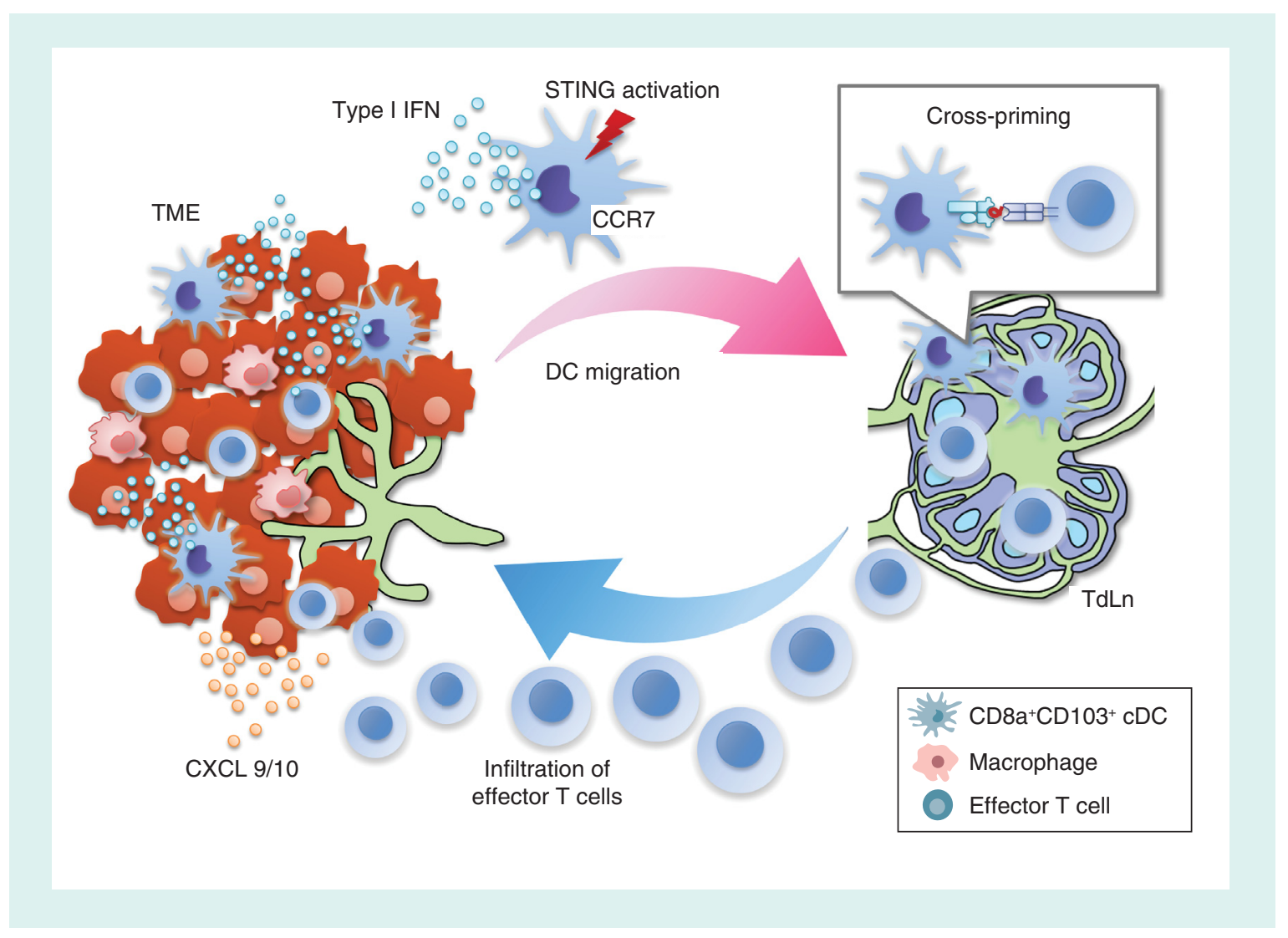

Figure 1. Loop for spontaneous T-cell responses against tumors. Antigen-presenting cells (APCs), especially $\mathrm{CD} 8 \alpha^{+} \mathrm{CD} 103^{+}, \mathrm{CDCs}$, engulf tumor cells, and induce the production of type I IFNs and migration to TdLN by activation via the STING pathway. DC migration is dependent on the CCR7 expressed on DCs. In the tumor-draining lymph node (TdLN), CD8 $\alpha^{+} \mathrm{CD} 103^{+} \mathrm{CDCs}$ then prime T cells. The activated T cells (effector T cells) infiltrate into the TME via the CXCL9/CXCL10 gradient.

CCR7: C-C chemokine receptor type 7; CDC: Conventional DC; CXCL9: C-X-C motif chemokine ligand 9; DC: Dendritic cell; TME: Tumor microenvironment.

based on several markers, including CD8 $\alpha, \mathrm{CD} 4, \mathrm{CD} 11 \mathrm{~b}$ and $\mathrm{CD} 103$. Batf3 $3^{--}$mice showed a selective loss of $\mathrm{CD} 8 \alpha^{+} \mathrm{cDCs}$ and cDCs obtained from Batf $3^{-/-}$mice were defective in cross-presentation [8]. Batf $3^{-/-}$mice lacked the ability to reject tumors against immunogenic tumors and the infiltration of cytotoxic T-lymphocytes (CTL) into the TME [8]. These results indicate that CD8 $\alpha^{+} \mathrm{cDCs}$ have a critical role in CTL-mediated antitumor responses. Moreover, the infiltration of $\mathrm{CD} 8 \alpha^{+}$cDCs into TME and the subsequent CTL mediated antitumor responses require type I IFN signaling [9]. That is, type I IFNs must signal CD $8 \alpha^{+} \mathrm{cDCs}$ for the optimal priming of CTL.

Recent studies have further characterized the DCs subset that initiates the innate immune sensing in TME and the findings indicate that $\mathrm{CD} 8 \alpha^{+} \mathrm{CD} 103^{+} \mathrm{cDC}$ driven by Batf3 is responsible for the transport of tumor antigens from TME to tumor-draining lymph nodes (TdLN) and $\mathrm{CD} 8^{+}$T-cell priming [11-13] (Figure 1). The $\mathrm{CD} 8 \alpha^{+} \mathrm{CD} 103^{+} \mathrm{cDCs}$ migration from TME to TdLN requires the C-C chemokine receptor type 7 [12]. Interestingly, the $\mathrm{CD} 8 \alpha^{+} \mathrm{CD} 103^{+} \mathrm{cDC}$ are involved in, not only direct $\mathrm{CD} 8^{+}$T-cell priming, but also antigen hand-off to resident APCs in TdLN [12]. On the other hand, CD169+ macrophages, lymph node (LN)-resident APCs, also dominate the activation of CTL, but not migratory DCs and LN-resident DCs [14]. This contradiction is due to differences in the status of tumor antigens. When dead tumor cells were injected, they were transported via lymphatic flow, during which $\mathrm{CD} 169^{+}$macrophages in the draining LN phagocytose them and subsequently crosspresent the antigen peptide to $\mathrm{CD} 8^{+} \mathrm{T}$ cells [14]. In the case of $\mathrm{CD} 8^{+} \mathrm{T}$-cell priming driven by dead tumor cells, migratory DCs and LN-resident DCs were not essential for cross-presentation. Meanwhile, in the immunogenic tumor-bearing mice, namely intact tumor cells, no tumor cell loading by $\mathrm{CD} 169^{+}$macrophages was observed, while the $\mathrm{CD} 8 \alpha^{+} \mathrm{CD} 103^{+}$cDCs were observed to engulf the tumor cells [12]. 
In addition to the engulfment of tumor antigens by the $\mathrm{CD} 8 \alpha^{+} \mathrm{CD} 103^{+} \mathrm{cDCs}$ in TME, a maturation process of $\mathrm{CD} 8 \alpha^{+} \mathrm{CD}_{103}{ }^{+} \mathrm{cDCs}$ is required for migration to TdLN. The $\mathrm{CD} 8 \alpha^{+} \mathrm{CD} 103^{+} \mathrm{cDCs}$ recognize cytosolic DNA derived from the engulfed tumor cells by the cGAS-STING, also as known as the TMEM173), resulting in maturation and the production of type I IFNs [3,15]. The cGAS binds to the cytosolic DNA and catalyzes the synthesis of cGAMP from ATP and GTP [16-20]. cGAMP functions as a second messenger that binds to STING. Stimulation of STING triggers a signal of the TBK1-IRF3 pathway, leading to the production of type I IFN and the maturation of APCs (Figure 1). Details of the cGAS-STING pathway have been reviewed elsewhere [19,20]. Moreover, other cyclic dinucleotides, including cyclic di-GMP (c-di-GMP) and cyclic di-AMP, also bind to STING [21]. Recent reports clearly show that the STING pathway is essential for innate immune sensing against immunogenic tumors [22,23]. STING and IRF3 were required for $\mathrm{CD}^{+}$T-cell priming in immunogenic B16.SIY melanomas [22]. Interestingly, $\mathrm{CD} 8^{+} \mathrm{T}$-cell priming was not required other innate immune pathway such as toll-like receptors. Moreover, the cGAS-STING pathway has an essential role as an antitumor effect of immune checkpoint therapy (anti-CTLA-4, anti-PD-1 and anti-PD-L1) [22,24]. The stimulation of the STING pathway in DCs also triggers the production of C-X-C motif chemokine ligand 9 (CXCL9) and CXCL10. These chemokines, which are recognized by the C-X-C motif chemokine receptor 3 on $\mathrm{CD}^{+} \mathrm{T}$ cells, are critical for the recruitment of effector T cells into TME [25]. The major source of CXCL9 and CXCL10 is the subset of CD $8 \alpha^{+}$CD103 ${ }^{+}$cDCs $[3,15]$. The effector T cells migrated via CXCL9/CXCL10 to TME attack tumor cells (Figure 1). Taken together, the sequence of spontaneous immune responses against immunogenic tumors protects us from invasion by cancer (Figure 1). That is, turning the cycle of antitumor responses can be effective for tumor rejection. Some aspects of the cycle are problematic in cancer patients. The cycle shown in Figure 1 can likely be fundamental, when we eventually understand the problems in the cancer patients, select appropriate therapies, and also design nano DDS for cancer immunotherapy.

\section{Combination therapy with immune checkpoint inhibitors}

Even if the infiltration of effector T cells into TME is induced through the above mechanism, tumors sometimes become clinically evident. Tumors cleverly escape from immune systems via several mechanisms. A typical example of such an escape mechanism is the suppression of effector T cells via PD-L1 expression. IFN- $\gamma$, produced by tumor-infiltrating T cells, upregulates PD-L1 expression on the tumor cells [26-28]. The PD-L1 on the tumor cells binds to the PD-1 on the tumor-infiltrating T cells, resulting in the dysfunction of T cells. However, in this case, anti-PD-1 monotherapy can be effective [29,30]. Anti-PD-1 reinvigorates tumor-infiltrating $T$ cells by inhibiting the PD-1/PD-L1 engagement. Recent reports show that the reinvigoration of tumor-associated macrophage (TAM) is also involved in the antitumor effect caused by PD-1/PD-L1 blockade [31]. PD-1 expression on TAM was reported to be correlated negatively with phagocytic activity against tumor cells, and PD-1/PD-L1 blockade increased the phagocytic activity of TAM and antitumor effects. For example, approximately $38 \%$ of advanced melanoma patients are included in this status of TME [29,30]. That is, more than $60 \%$ of patients have another immune status in TME and are resistant to anti-PD-1 monotherapy. To improve the response rate and to overcome this resistance, combination therapies with conventional therapy or other immune checkpoint inhibitors are currently being evaluated in ongoing clinical trials.

Inhibitory receptors other than PD-1 also participate in immune suppression in TME. This includes, for example, TIM3, CTLA-4 and LAG3. TIM3 is selectively expressed on Th1 and CTL [32]. The C-type lectin galectin-9 is a ligand of TIM3, and TIM3/galectin-9 engagement negatively regulates T-cell functions [33]. Although, a TIM3 blockade alone showed antitumor effects that were similar to a PD-1 blockade, a combination of anti-TIM3 with anit-PD-1 was found to be more effective for tumor regression [34,35]. Phase I and II trials using an anti-TIM3/antiPD-1 combination is in the planning stage (NCT02817633, NCT02608268). CTLA-4 is also a checkpoint molecule that is expressed on effector T cells and binds to CD80 or CD86, leading to T-cell dysfunction. AntiCTLA- 4 showed a drastic antitumor effect [36], and became the first approved immune checkpoint inhibitor in 2011. The effect of a combination of anti-CTLA- 4 with anti-PD-1 has been already evaluated in several clinical trials [37,38]. However, an increase in adverse events was observed when this combination was used [38]. LAG3 is type I transmembrane protein expressed on activated T cells and NK cells, and is highly structurally homologous to CD4 [39,40]. Thus, the ligand of LAG3 is a MHC class II molecule, and liver sinusoidal endothelial cell lectin (LSECtin) and galectin-3 are also alternative ligands [40]. In a mouse model, the treatment of both anti-LAG3 and anti-PD-1 showed an antitumor effect against established tumors that were largely resistant to treatment with a 
single antibody [41]. Combinations of anti-LAG3 with anti-PD-1 are currently being evaluated in several clinical trials (NCT01968109, NCT02061761, NCT02750514, etc).

Indoleamine 2,3-dioxygenase (IDO) also functions as an immune escape mechanism [42]. IDO is a metabolic enzyme that converts tryptophan into kynurenine. IDO is expressed in human cancer, and IDO expression in immunogenic mouse tumors enhanced their growth [43]. The depletion of local tryptophan by IDO activates the stress-response kinase GCN2, and GCN2 activation in T cells induces the inhibition of their proliferation and the differentiation from naive $\mathrm{CD}^{+} \mathrm{T}$ cells to regulatory $\mathrm{T}$ cells (Treg) [42,44]. Most studies have shown that a high expression of IDO in TME or TdLN is an adverse prognostic factor [45-50]. IDO, by its nature, can function in a counter-regulatory manner, and is induced in response to IFN production [42]. In TME, the induction of type I IFNs by the stimulation of the STING pathway appear to be an inducer of IDO. Antigenicity associated with TME is involved in the IDO induction by the STING pathway [51]. Antigenicity defines the capacity of an antigen peptide to specifically bind to lymphocyte receptors [2]. That is, tumor cells either express tumor antigens cross-presented by APCs or do not. Stimulation of the STING pathway promotes antitumor immunity in the presence of tumor antigens (high antigenicity), while it induces IDO, suppresses the antitumor immunity and promotes tumorigenesis in the case of low antigenic TME [42,51]. As pointed out above, IDO is one of the important mechanism for Treg induction in TME. Treg is a major suppressive mechanism in TME, and details regarding the role of Treg in cancer immunity are reviewed elsewhere [52]. IDO-mediated GCN2 activation in Treg inhibits the mTOR/Akt signal pathway that suppresses Treg functions, leading to the upregulation of FoxO3a, PD-1, PTEN [42]. Consequently, a signaling loop from PD-1 to PTEN stably maintains the highly suppressive status. Thus, the inhibition of IDO represents a potential strategy for reprograming the immune status in TME. Combinations of IDO inhibitors with chemotherapy, immune checkpoint inhibitors, or vaccines are currently being evaluated in clinical trials [42]. At the American Society of Clinical Oncology 2017 meeting, promising clinical responses regarding the use of the combination of an IDO inhibitor with an anti-PD-1 were reported. A Phase III study of the use of a combination of IDO inhibitor with anti-PD-1 are currently in the planning stage (NCT02752074).

In contrast to T-cell inflamed TME, non-T-cell inflamed TME is observed in cancer patients. More than $40 \%$ of melanoma patients are classified to the immune status in TME and are predicted to have a very poor prognosis [53]. In this situation, anti-PD-1 monotherapy would most likely fail, because of the lack of effector T-cell infiltration. Thus, in current immunotherapy, non-T-cell inflamed TME can be the most serious problem and overcoming the immune status in TME can be considered to be the next big hurdle. Non-T-cell infiltration to TME can be due to the suppression of innate immune sensing and the recruitment of effector $T$ cells. The comparative gene expression profiling of human melanomas between non-T-cell inflamed and T-cell inflamed revealed that the activation of the WST/ $\beta$-catenin signaling pathway, a melanoma-cell-intrinsic oncogenic pathway, contributed to a lack of T-cell infiltration in melanoma cases [54]. The activation of the WST/ $\beta$-catenin signaling pathway in melanoma cells results in the defective production of CCL4 from melanoma cells, leading to the defective recruitment of $\mathrm{CD}_{103}{ }^{+}$DCs into TME. Consequently, the innate sensing of tumor cells by DCs does not happen, and subsequent T-cell priming/recruitment is also impaired. An anti-CTLA-4-anti-PD-1 combination showed no therapeutic effect against WST/ $\beta$-catenin signaling activated tumors, while this resistance was reversed by the intratumoral injection of Flt3 ligand-induced DCs. CXCL9 and CXCL10 are important ligands that are responsible for the infiltration of effector T cells into TME. The genetic methylation of CXCL9 and CXCL10 loci represses the production of these chemokines from tumor cells, and subsequently impairs T-cell infiltration into TME [55]. The methylation was associated with the enhancer of EZH2-mediated histone $\mathrm{H} 3$ lysine 27 trimethylation and DNMT1-mediated DNA methylation. In this study, the authors showed that the administration of a DNMT-1 inhibitor, 5-aza-2'-deoxycytidine, restored the loss of therapeutic antitumor effect by the immune checkpoint inhibitor.

Conventional and targeted anticancer drugs are also attractive partners for combination therapy with immune checkpoint inhibitors. Because an accumulating body of evidence indicates that the therapeutic effect caused by some anticancer drugs involve, not only direct cell killing, but also antitumor effects by the immune system [56]. Several mechanisms were extensively discussed in this review [56], for example, the enhancement of tumor antigen release, the release of endogenous adjuvants, and the deletion of suppressor cells. The treatment of oxaliplatin/cyclophosphamide elicited the infiltration of effector T cells against Kras/Trp 53 mutant tumors lacking T-cell infiltration, namely tumors that were resistant to immune checkpoint inhibitors [57]. In addition, the oxaliplatin/cyclophosphamide induced infiltration of T cells were sensitized to anti-PD-1/anti-CTLA- 4 therapy. These results indicate that when combined with chemotherapy this approach can be a potent candidate for over- 
coming non-T-cell inflamed tumors. Recent clinical trials clearly show the incredible effect of using a combination of chemotherapy with anti-PD-1 [58]. The combination therapy, carboplatin and pemetrexed with pembrolizumab (anti-PD-1), against advanced, nonsquamous NSCLC achieved 55\% objective response. This combination therapy was approved for use by the US FDA in May 2017 as a first-line therapy for patients with metastatic nonsquamous NSCLC.

In contrast to efficacy, immunotherapy shows distinct adverse effects that are different from those associated with normal cancer therapy. $T$ cells undergo natural central selection in the thymus, autoreactive $T$ cells in the periphery are controlled by Tregs and immune checkpoint molecules. However, when the regulation of the immune system is disrupted by immunotherapy, aberrations of immune homeostasis lead to inflammation mainly involving T-cell infiltration in the whole body and in individual organs, resulting in autoimmune-like symptoms. This type of toxicity is defined as an immune-related adverse event (irAE). Major irAEs are disorders of the skin, GI tract, liver and endocrine system. Cytokine storm is one such serious disorder. Details of irAE have been reviewed elsewhere [59,60]. The irAE disorder appears to occur in effective immunotherapy against cancer. Although, cancer vaccines generally show minimal toxicity, adaptive cell therapy and immune check point inhibitors are likely to show a high incidence of irAE and serious side effects can sometimes occur [59]. The management of irAE is a very important issue in cancer immunotherapy. Furthermore, when irAE is used in combination therapy, side effects are generally more severe than those in monotherapy [38]. Thus, it is necessary to thoroughly monitor the dosage and administration intervals.

Collectively, a combination therapy using immune checkpoint inhibitors in conjunction with other cancer therapies can be effective and promising for improving the response rate and overcoming resistance in nonresponsive patients with checkpoint inhibitor monotherapy. On the other hand, an increase in adverse effects is the issue of most concern, because the current combination enhances systemic immune activities and suppresses systemic immune regulation. Controlling the biodistribution of a drug by nano DDS technology would contribute to the reduction of adverse effects and the enhancement of positive effects. Moreover, nano DDS technology can provide a more precisely directed combination therapy associated with complex immune status in TME.

\section{Nano DDS in cancer immunotherapy}

Nano DDS, namely nanoparticles, have been used to enhance immune responses since the skeptical era against cancer immunotherapy, and has made important contributions to the development of effective cancer immunotherapies. Nanoparticle formulations such as micelles, liposomes, LNPs, polyplexes and emulsions, are frequently used for assisting immunotherapy $[4,5]$. Nanoparticles increase the water dispersibility of immune-functional molecules, protect them from degradation and efficiently deliver them to tissues, cells or organelles, leading to drastic enhancements in antitumor immunity.

To induce effective antitumor immune responses, delivering antigens to APCs is one of the major and established strategies. Generally, exogenous antigens taken up via endocytosis are degraded in lysosomes and the peptides derived from the antigens are presented on the MHC class II molecule (MHC-II), resulting in antigen presentation to $\mathrm{CD}^{+}$ $\mathrm{T}$ cells [61]. On the other hand, endogenous antigens in the cytosol are degraded by proteasomes and the peptides are presented to the MHC class I molecule (MHC-I), resulting in the antigen being presented to $\mathrm{CD} 8^{+} \mathrm{T}$ cells. Thus, antigen presentation on MHC-I is crucial for $\mathrm{CD}^{+}$T-cell priming. To achieve MHC-I antigen presentation, exogenous antigens must be delivered to the cytosol, because tumor antigens are almost all exogenous in nature. Fusogenic liposomes composed of dioleoyl phosphatidylethanol amine (DOPE) was found to enhance antigen presentation on MHC class I molecules, namely by cross-presentation [62]. Furthermore, the use of a combination of cell-penetrating peptides with fusogenic liposomes resulted in a drastic enhancement of cellular uptake and MHC-I presentation [63]. The octaarginine (R8) peptide, a type of cell-penetrating peptides, was used to modify the surface of DOPE-based fusogenic liposomes by using stearylated R8 (STR-R8). In in vitro experiments, the R8-modified liposome (R8-Lip) was taken up by most mouse DCs via macropinocytosis, delivered the encapsulated substance to the cytosol, and induced specific MHC-I antigen presentation. Interestingly, the R8-Lip promoted MHC-I antigen presentation not only by delivering the antigen to the cytosol, but also by enhancing the C-terminal trimming of the antigen peptide via proteasomes [64]. In addition to liposomes, similar strategy was used to achieve efficient cross-presentation in micelles and poly(lactic-co-glycolic acid) (PLGA) nanoparticles [65,66]. Collectively, it appears that the cytosolic delivery of antigens by a nano DDS can induce much more effective cross-presentation compared with only the antigen $[5,67]$. 
Another factor for the effective induction of antitumor immunity is the stimulation of APCs by adjuvants. If the stimulation by an adjuvant is absent, DCs with internalized antigens result in immune tolerance or suppression. Ligands for pattern-recognition receptors associated with innate immunity are currently used as an adjuvant. These ligands induce the maturation of DCs and cytokine production [68]. In particular, ligands for nucleic acidsensing pathways are expected to function as a cancer adjuvant [69]. Polyinosine-polycytidylic acid (polyI:C, double stranded RNA, TLR3/Mda5 ligand), CpG oligodeoxynucleotides (CpG-ODNs, DNA containing unmethylated CpG motifs, the TLR9 ligand), and DNA or cyclic dinucleotides (a STING pathway ligand) are frequently used for this purpose. However, these adjuvants by themselves cannot internalize into cells, because they are highly negatively charged and are degraded by nucleases. Thus, when the nucleic acid based ligands are applied to cancer adjuvants, a nano DDS can be a useful technology. Because TLR3 and TLR9 are present in endosomes, main strategy of nano DDS is to promote the cellular internalization of polyI:C and CpG-ODNs [70-72]. In addition to increasing the cellular internalization of adjuvants, we discuss the importance of nano DDS design with regard to the topology of adjuvants [73]. We prepared two types of nanoparticles; R8-Lip encapsulating polyI:C and ovalbumin (OVA) (R8-Lip/PIC/OVA); the complex of STR-R8, polyI:C, and OVA (R8/PIC/OVA-Com). Both nanoparticles had similar sizes and both were positively charged. Interestingly, when mice were immunized with the R8-Lip/PIC/OVA, a much stronger OVA-specific CTL induction was observed and antitumor effects against the E.G7-OVA tumor were larger than that of the R8/PIC/OVA-Com. This difference can be attributed to the following reasons. In the case of R8-Lip/PIC/OVA, polyI:C in the aqueous phase of the liposome would be expected to be free form, while the polyI:C in the R8/PIC/OVA-Com may not be, due to electrostatic binding with STR-R8. Thus, it is suggested that the polyI:C in R8-Lip/PIC/OVA would easily access the receptors compared with that in R8/PIC/OVA-Com. Another reason is that the polyI:C in R8-Lip/PIC/OVA might be recognized by TLR3 in endosomes and Mda5 in the cytosol, because the R8-Lip can deliver encapsulated substances to the cytosol [63]. Clinical applications of polyI:C are predicted to be difficult due to the development of systemic toxicity, while a new TLR3-specific adjuvant without systemic toxicity, ARNAX, was developed [74]. The ARNAX induced effective antitumor immunity by NK cells and CTL [74], and also relieved anti-PD-L1 resistance [75]. Clinical applications of ARNAX and the combination with nano DDS are expected in the future. The development of CpG-ODNs as a cancer adjuvant is being actively pursued [76]. A novel nanoparticle form of K type CpG ODN, K3-schizophyllan (K3-SPG), was recently reported [77]. K3-SPG efficiently induced both humoral and cellular immune responses, especially CTL by accumulating to the dLN.

Recent progress in the development of cancer adjuvant, agonists of the STING pathway have attracted considerable interest. The activation of the STING pathway is highly related to the induction of antitumor immunity, as pointed out above. STING agonists are recognized by cGAS or adaptor molecules in the cytosol. Thus, applying STING agonists as a cancer adjuvant represents a bigger hurdle than polyI:C and CpG-ODNs. Our group first reported on the successful development of a STING agonist (c-di-GMP) loaded nanoparticle for use as a cancer adjuvant [78]. The c-di-GMP binds to DDX41, a cytosolic ATP dependent RNA helicase, and forms a complex with STING, resulting in the activation of the TBK1-IRF3 pathway [21,79]. We loaded YSK05, a synthetic lipid-based LNP (YSK05-LNP) with c-di-GMP for the efficient cytosolic delivery of c-di-GMP [78]. YSK05 has pH-responsive and high fusogenic properties, which permits efficient endosomal escape [80]. Treatment with the c-di-GMP loaded YSK05-LNP (cdGMP/YSK05-LNP) efficiently induced type I IFN from RAW264.7 cells compared with that of c-di-GMP loaded conventional transfection reagent, which was dependent on the TBK1-IRF3 pathway [78]. Immunization of mice with OVA with the cdGMP/YSK05-LNP resulted in the induction of an OVA-specific CTL and antitumor effect against EG.7-OVA tumors, suggesting that the cdGMP/YSK05-LNP has the potential for function as a potent inducer of antigen-specific tumor immunity [78]. Meanwhile, a part of cancer cells escape from elimination by CTL by changing the MHC-I expression [81]. In this case, NK cells can function as a major reliable effector cell against the cancer cells, such as in cases of malignant melanomas. Thus, we investigated the effect of cdGMP/YSK05-LNP on NK cells [82]. The intravenous administration of cdGMP/YSK05-LNP to mice activated NK cells in the spleen and prevented tumor growth of B16-F10 melanomas in a lung metastasis mouse model. This antitumor effect was dependent on NK cells, and NK cells infiltrated to the lung with B16-F10 melanomas. The findings suggest that cdGMP/YSK05-LNP induced MHC-I non-restricted antitumor immunity via NK cells. Subsequently, a c-di-GMP loaded nanoparticle targeting LN was recently reported [83]. The c-di-GMP was loaded with $150 \mathrm{~nm}$ sized LNP particles modified with $5 \mathrm{~mol} \%$ of a PEGylated lipid. The LNP accumulated to the LN and was taken up by APCs in the LN. In addition, the treatment of LNP with OVA strongly induced the production of OVA-specific $\mathrm{CD}^{+} \mathrm{T}$ cells and antitumor effects against EG.7-OVA tumors. These 
results indicate that $\mathrm{LN}$-targeting by nanoparticles is a useful strategy for maximizing the functions of STING agonists. Furthermore, a recent report described a unique nano DDS for activating the STING pathway [84]. The polymer-based nanoparticle, PC7A-NPs, were $29 \mathrm{~nm}$ in diameter, and subcutaneously administrated PC7A-NPs efficiently accumulated to the LN, leading to the production of type I IFN and cross-presentation. Interestingly, the induction of immune responses was dependent on the STING pathway, and a PC7A polymer found to directly bind to STING. The PC7A-NPs led to the potent inhibition of tumor growth against melanomas, colon cancer and human papilloma virus-E6/E7 tumor models. Moreover, the combination of antigen peptide loaded PC7A-NPs with anti-PD-1 exhibited a substantial synergistic antitumor effect in B16-OVA melanomas and a TC-1 tumor model. On the other hand, anti-PD-1 monotherapy showed no antitumor effects in either of these models. The low efficiency can be due to the low expression of PD-L1 on tumor cells. Although it is our conclusion that treatment with antigen peptide loaded PC7A-NPs efficiently induced antigen-specific CTL, enhanced CTL infiltration into TME, thus establishing PD-1/PD-L1 suppression, resulting in the realization of an anti-PD-1 effect.

We achieved a reversed-translational approach in the development of cancer immunotherapy by using the nano DDS. The intravesical instillation of a live Bacillus Calmette-Guerin (BCG) is the gold standard therapy against superficial bladder cancer and carcinoma in situ, in addition to a vaccine against tuberculosis [85]. BCG therapy can induce a very effective antitumor immunity and is a quite successful cancer immunotherapy. Thus, BCG therapy would be expected to be applicable to other types of cancer. However, applying it to other cancers is quite impossible, because of serious adverse effects associated with the use of live mycobacteria [86]. Such side effects pose a significant concern even if locally intravesical instilled. Therefore, we considered the possibility of a cancer immunotherapy based on an artificial BCG by loading a nano DDS with immune-simulative components of BCG. The BCG cell wall skeleton (BCG-CWS), the main immune active component of BCG [87], would be a potent drug instead of live BCG. But, BCG-CWS cannot be used in a drug formulation, because of its unfavorable physical characteristics. BCG-CWS in a hydrophilic solution shows heterogeneity in the size of tens of micrometers [88]. These unfavorable characters hamper the delivery of BCG-CWS to target cells such as bladder cancer cells and APCs. However, we succeeded in developing a novel packaging method, liposome evaporated via emulsified lipid (LEEL) method, that permits BCG-CWS to be encapsulated into a sub-200 nm sized LNP [89]. The LNP encapsulating BCG-CWS (CWS-LNP) achieved a high uniform and water-dispersed character. CWS-LNP was efficiently taken up by mouse bladder cancer cells in vitro, and the CWS-NP treatment showed a significant antitumor effect against mice bearing mouse bladder tumors [89]. This anti-bladder tumor mechanism was similar to that of BCG [90]. Moreover, the intravesical administration of CWS-LNP to a carcinogen-induced urinary bladder cancer rat model caused the tumors to shrink. Since the rat model is similar to the actual clinical conditions, CWS-LNP would be expected to be a potent candidate for an anti-bladder cancer immunotherapeutic drug. In addition, in contrast to BCG, CWS-NP can be applied to other types of cancer, because of its non-infectiveness. Nano DDS has the potential for expanding the application of clinical-used immunotherapy.

As partly pointed out above, the LN-targeting of antigens and/or adjuvants is a potent strategy for enhancing antitumor immunity and the nano DDS can largely contribute to the success of such a system. Most small molecules are primarily drained from the interstitial space via blood capillaries, while macromolecules such as proteins generally enter into the lymphatic system. That is, the lymphatic entry of molecules is dependent on the size. Small molecules $<10 \mathrm{~nm}$ in size are primarily transported via blood capillaries rather than lymphatic capillaries [91]. On the other hand, particles $>100 \mathrm{~nm}$ are poorly transported in lymphatics [91]. Macromolecules with weights of 20-30 kDa and particles with sizes of 10-100 nm are suitable for entry into lymphatic vessels [91]. Reddy et al. clearly showed the effect of nanoparticle size on lymphatic transport [92]. The efficiency of lymphatic transport between $100 \mathrm{~nm}$ pluronic-stabilized polypropylene sulfide nanoparticles (100 nm-NPs) and $25 \mathrm{~nm}-\mathrm{NPs}$ were compared. As a result, $25 \mathrm{~nm}$ NPs were highly efficiently transported via lymphatic vessels into the LN, whereas the efficiency of transport of $100 \mathrm{~nm}$ NPs was only 10\%. In addition to PC7A-NPs [84], polymeric nanoparticles with sizes of $30 \mathrm{~nm}$ also successfully delivered the adjuvant to DCs in the LN [93]. Liu et al. reported the $\mathrm{LN}$-targeting cancer vaccine based on an albumin hitchhiking approach, which is a pioneering study that uses LN-targeted delivery for enhancing vaccinations [94]. The authors linked the antigen or adjuvant to a lipophilic albumin-binding tail by a solubility-promoting polar polymer chain. This system resulted in a marked increase in LN accumulation, a 30-fold increase in T-cell priming and a strong antitumor effect. A unique nano DDS, high-density lipoprotein-mimicking nanodiscs, can markedly improve antigen/adjuvant co-delivery to the LN, cross-presentation and antitumor immunity [95]. The nanodiscs also induced a synergistic antitumor effect against B16-F10 melanoma cells by the combination therapy with anti-PD-1 and anti-CTLA-4. The mechanism in this 


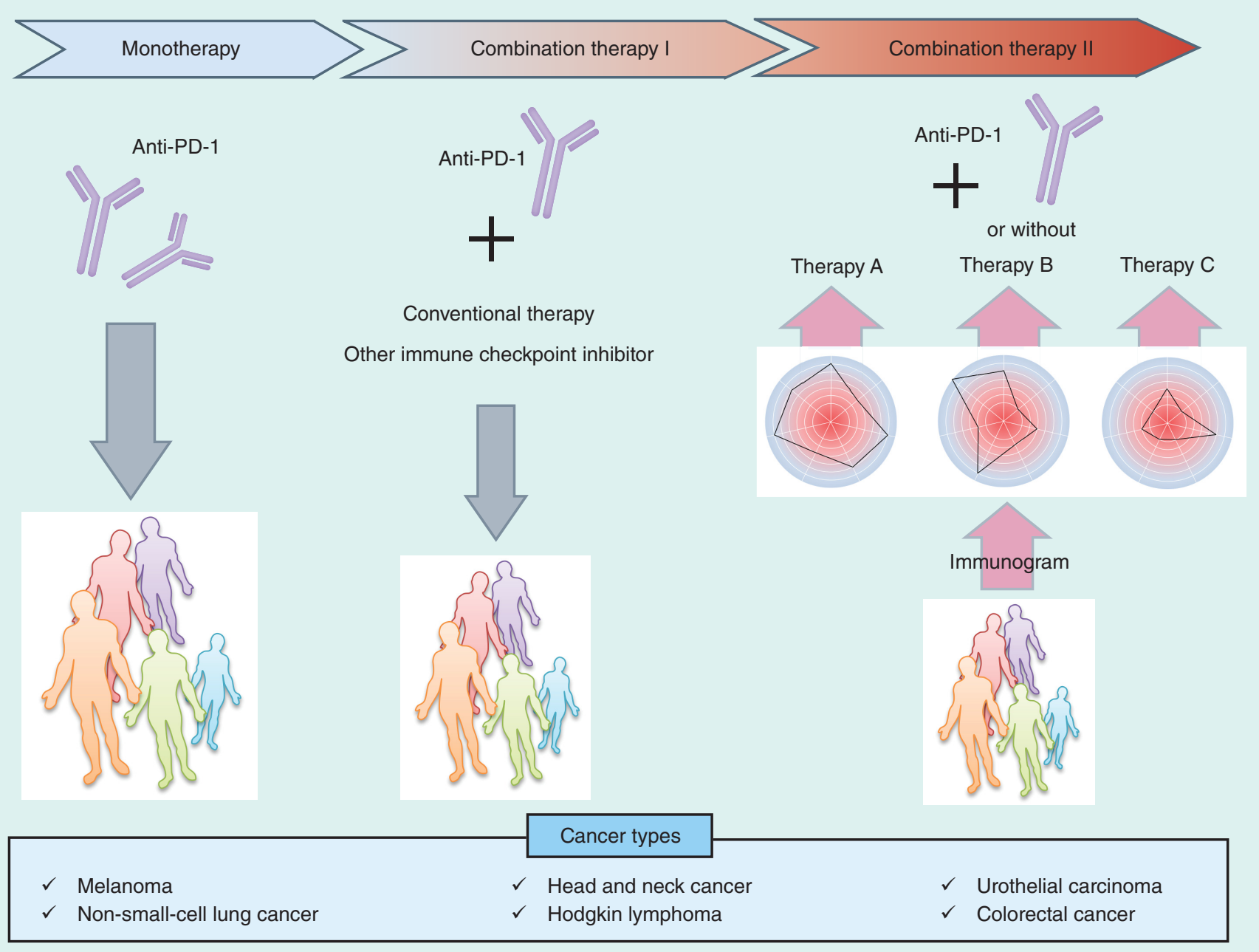

Figure 2. Change in development strategy for anti-PD-1-based cancer immunotherapy. Verification of anti-PD-1 monotherapy against various cancer types has largely been completed. In current combination therapy (combination therapy I), a combination of anti-PD-1 with conventional cancer therapy or other immune checkpoint inhibitors is being evaluated for use against several cancer types. In the near future, the combination therapy will change drastically into a therapy in which optimal drugs for an individual patient's immune status in TME combined with classification by means of an immunogram (combination therapy II).

TME: Tumor microenvironment.

case appears to be similar to that for PC7A-NPs. The nanodisc-induced suppression of CTL was unleashed by immune checkpoint inhibitors. Taken together, LN-targeting by nano DDS represents a promising strategy for cancer immunotherapy.

\section{Future perspective}

Since, the development of anti-PD-1, the validity of anti-PD-1 against various cancer types was rapidly verified and its verification in now largely completed (Figure 2, monotherapy part). The current mainstream is combination therapy with anti-PD-1 to overcome anti-PD-1 resistance. The feature of current combination therapies are simply a combination of existing treatments mainly to enhance overall effectiveness (Figure 2, combination therapy I part). It is comprehensive, but not strategic. However, based on the clinical data obtained to date far and findings related to tumor immunology, the strategic combination therapy considering the immune status of TME is being steered (Figure 2, combination therapy II part). For example, we have selected targets only for cancer types so far, but in the future, it is expected that more specific targets will be selected, such as patients who are anti-PD-1 resistant or without T-cell infiltration. To visually understand immune status in TME, the use of cancer immunogram is proposed [96]. The cancer immunogram is a radar plot depicting some parameters that characterize aspects of the 


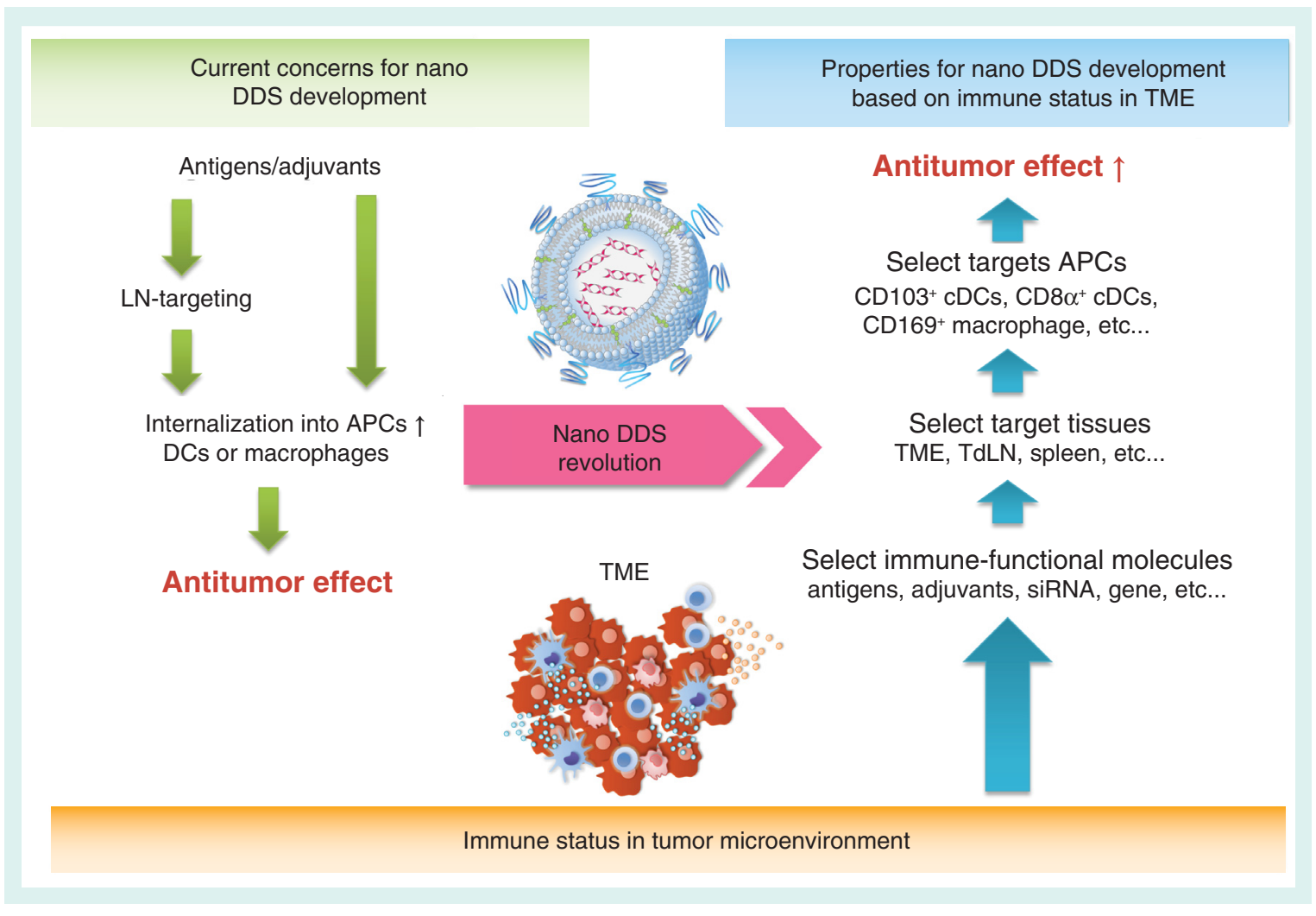

Figure 3. Revolution of nanotechnology-based drug-delivery system. Current concerns for nano DDS development focuses on efficiently delivering cargoes to the LN and APCs, and enhancing antitumor activity. However, in order for nano DDS to contribute to combination therapy in the future (Figure 2, combination therapy II), the strategy for using nano DDS will need to be changed. Similar to combination therapy II, cargoes, target tissues and APCS are selected based on the individual patient's immune status in the TME, and nano DDS is designed to achieve them. APC: Antigen-presenting cell; CDC: Conventional dendritic cell; DDS: Drug-delivery system; LN: Lymph node; TdLN: Tumor draining lymph node; TME: Tumor microenvironment.

immune status in TME (Figure 2, combination therapy II part). The further the point is from the center of the circle, the more desirable it is, and the closer it is to the center, the more undesirable it is. The development of biomarkers and methods for creating a cancer immunogram has not been established yet, but it will surely become a very useful tool in the future. Collectively, considering the immune status in TME must be quite important in terms of managing cancer immunotherapy. That is, the same can be said for nano DDS development in cancer immunotherapy.

Numerous nano DDS particles in the field of cancer immunotherapy have been reported. However, the design of a nano DDS based on immune status in the TME has never been considered. The current nano DDS largely focuses on the promotion of antigen presentation, the activation of APCs, targeting DCs, targeting lymph tissues (Figure 3). The functions are also analyzed by routine methodology and tumor-bearing mice. Of course, evaluating the validity of the current nano DDS in combination therapy with immune check point inhibitors is necessary for advancing the development of cancer immunotherapy. The current nano DDS preparations can promote the efficacy of immune checkpoint inhibitors and permit their dosage to be decreased. Meanwhile, recent advances in tumor immunology requires strategic combined immunotherapy procedures based on the immune status in the TME. Thus, it will be necessary to develop nano DDS capable of responding to various types of immune status in TME (Figure 2). Where should immune-functional molecules, such as antigens and adjuvants, be delivered to, in the case of a T-cell-inflamed tumor? What about the case of T-cell-noninflamed tumors? Which cell should immune-functional molecules be delivered to, migratory DCs or resident DCs? It will be necessary to systematize the relationship between a delivery strategy based on the use of a nano DDS and the immune status in the TME. Antigens and STING agonists may be delivered to $\mathrm{CD} 8 \alpha^{+} \mathrm{CD} 103^{+}$cDCs in the TME in the case of T-cell 
inflamed tumors. In the case of T-cell-non-inflamed tumors, T-cell infiltration into the TME may be first promoted by delivering antigens/adjuvants to $\mathrm{CD} 8 \alpha^{+}$cDCs in the TdLN. In addition to delivering antigens/adjuvants to APCs, controlling the function of other immune cells, such as T cells, NK cells, B cells, etc., would be required to overcome the immune suppression in the TME. Controlling such a function at the gene level could be a potent strategy, because it is difficult to cover all immuno-suppressive factors by antibodies and small molecules. We recently succeeded in developing a novel LNP for introducing small interfering (siRNA) to immune cells [97,98]. The main component of the LNP is YSK12-C4, a lipid having cationic properties and a high fusogenic activity. The siRNA loaded LNP was greatly effective in silencing against the target gene in mouse DCs and human immune cell lines (Jurkat, THP-1, KG-1, NK92), compared with conventional siRNA transfection reagents. Therefore, our LNP would be expected to be a potent technology for use after immune checkpoint inhibitors for controlling the functions of immune cells to overcome immune suppression.

\section{Conclusion}

Over the past few years, cancer immunotherapy and tumor immunology have rapidly progressed. In the near future, precision combination immunotherapy will be performed based on analyses of the immune status in the TME of patients. Nano DDS has the potential to make a significant contribution to the success of this immunotherapy as a key technology. In addition to the enhancement of efficacy, nano DDS appear to be useful for decreasing irAEs. To achieve this, it will be necessary to revolutionize nano DDS in the field of cancer immunotherapy.

Financial \& competing interests disclosure

This work was supported, in part by JSPS KAKENHI Grant Numbers 17H03974 and 16K15102. We also appreciate MS Feather for this helpful advice in writing the English manuscript. The authors have no other relevant affiliations or financial involvement with any organization or entity with a financial interest in or financial conflict with the subject matter or materials discussed in the manuscript apart from those disclosed.

No writing assistance was utilized in the production of this manuscript.

\section{Executive summary}

\section{Spontaneous immune response in tumor microenvironments}

- $\mathrm{CD} 8 \alpha^{+}$conventional dendritic cells (cDCs) driven by the transcription factor Batf3, particularly $\mathrm{CD} 8 \alpha^{+} \mathrm{CD} 103^{+}$ $C D C s$, is crucial for the induction of antitumor responses against immunogenic tumors.

- After the engulfment of tumor cells in the tumor microenvironment (TME), $C D 8 \alpha^{+} \mathrm{CD} 103^{+} \mathrm{CDCs}$ produce type I IFN via activation of the STING pathway, and then migrate to tumor-draining lymph nodes for cross-priming to $\mathrm{CD}^{+}{ }^{+} \mathrm{T}$ cells.

- The activated $\mathrm{CD}^{+} \mathrm{T}$ cells infiltrate into the TME dependent on CXCL9/10, leading to the elimination of tumor cells.

Combination therapy with immune checkpoint inhibitors

- Combination therapy using anti-PD-1 with antibodies against other inhibitory receptors are currently being evaluated in several clinical trials.

- Combination therapy with anti-PD-1 combined with a IDO inhibitor is a promising approach.

- Combination therapy with anti-PD-1 combined with chemotherapy shows great synergic effects and has already approved by the US FDA.

Nano drug-delivery system in cancer therapy

- Nanoparticles efficiently deliver antigens and adjuvants to the antigen-presenting cells, leading to an enhanced cross-presentation, antigen-presenting cells maturation, and antitumor immunity.

- Nanoparticles loaded with a STING agonist represent a potential, next-generation adjuvant system.

- Lymph node targeting of antigens/adjuvants by nanoparticles are one of the potent strategies for cancer immunotherapy.

- The development of a nano drug-delivery system focused on the immune status in TME will be necessary.

\section{References}

Papers of special note have been highlighted as: $\bullet$ of interest; $\bullet \bullet$ of considerable interest.

1 Sharma P, Allison JP. The future of immune checkpoint therapy. Science 348(6230), 56-61 (2015).

- Review on the progress and perspectives related to immune chekpoint therapy.

2 Bronte V. Tumors STING adaptive antitumor immunity. Immunity 41(5), 679-681 (2014). 
3 Spranger S. Mechanisms of tumor escape in the context of the T-cell-inflamed and the non-T-cell-inflamed tumor microenvironment. Int. Immunol. 28(8), 383-391 (2016).

•. Review on the immune status in tumor microenvironments.

4 Kapadia CH, Perry JL, Tian S, Luft JC, DeSimone JM. Nanoparticulate immunotherapy for cancer. J. Control. Rel. 219 167-180 (2015).

5 Irvine DJ, Hanson MC, Rakhra K, Tokatlian T. Synthetic nanoparticles for vaccines and immunotherapy. Chem. Rev. 115(19), 11109-11146 (2015).

6 Dunn GP, Bruce AT, Ikeda H, Old LJ, Schreiber RD. Cancer immunoediting: from immunosurveillance to tumor escape. Nat. Immunol. 3(11), 991-998 (2002).

7 Dunn GP, Koebel CM, Schreiber RD. Interferons, immunity and cancer immunoediting. Nat. Rev. Immunol. 6(11), 836-848 (2006).

8 Hildner K, Edelson BT, Purtha WE et al. Batf3 deficiency reveals a critical role for CD8alpha+ dendritic cells in cytotoxic T cell immunity. Science 322(5904), 1097-1100 (2008).

-. First report showing that dendritic cells driven by Batf3 are crucial for the rejection of immunogenic tumors.

9 Fuertes MB, Kacha AK, Kline J et al. Host type I IFN signals are required for antitumor CD8+ T cell responses through CD8 $\{$ alpha $\}+$ dendritic cells. J. Exp. Med. 208(10), 2005-2016 (2011).

10 Merad M, Sathe P, Helft J, Miller J, Mortha A. The dendritic cell lineage: ontogeny and function of dendritic cells and their subsets in the steady state and the inflamed setting. Annu. Rev. Immunol. 31 563-604 (2013).

11 Broz ML, Binnewies M, Boldajipour B et al. Dissecting the tumor myeloid compartment reveals rare activating antigen-presenting cells critical for T cell immunity. Cancer Cell 26(5), 638-652 (2014).

-. The data presented in this article indicate that $\mathrm{CD}_{103}{ }^{+}$dendritic cells is a central player for spontaneous $\mathrm{T}$-cell responses against immunogenic tumors.

12 Roberts EW, Broz ML, Binnewies M et al. Critical role for CD103(+)/CD141(+) dendritic cells bearing CCR7 for tumor antigen trafficking and priming of T cell immunity in melanoma. Cancer Cell 30(2), 324-336 (2016).

13 Salmon H, Idoyaga J, Rahman A et al. Expansion and activation of CD103(+) dendritic cell progenitors at the tumor site enhances tumor responses to therapeutic PD-L1 and BRAF inhibition. Immunity 44(4), 924-938 (2016).

14 Asano K, Nabeyama A, Miyake Y et al. CD169-positive macrophages dominate antitumor immunity by crosspresenting dead cell-associated antigens. Immunity 34(1), 85-95 (2011).

15 Corrales L, Matson V, Flood B, Spranger S, Gajewski TF. Innate immune signaling and regulation in cancer immunotherapy. Cell. Res. 27(1), 96-108 (2017).

16 Ishikawa H, Barber GN. STING is an endoplasmic reticulum adaptor that facilitates innate immune signalling. Nature 455(7213), 674-678 (2008).

-. First report showing that STING is a DNA sensor in innate immunity.

17 Ishikawa H, Ma Z, Barber GN. STING regulates intracellular DNA-mediated, type I interferon-dependent innate immunity. Nature 461(7265), 788-792 (2009).

18 Sun L, Wu J, Du F, Chen X, Chen ZJ. Cyclic GMP-AMP synthase is a cytosolic DNA sensor that activates the type I interferon pathway. Science 339(6121), 786-791 (2013).

19 Barber GN. STING: infection, inflammation and cancer. Nat. Rev. Immunol. 15(12), 760-770 (2015).

20 Chen Q, Sun L, Chen ZJ. Regulation and function of the cGAS-STING pathway of cytosolic DNA sensing. Nat. Immunol. 17(10), 1142-1149 (2016).

21 Burdette DL, Monroe KM, Sotelo-Troha K et al. STING is a direct innate immune sensor of cyclic di-GMP. Nature 478(7370), 515-518 (2011).

22 Woo SR, Fuertes MB, Corrales L et al. STING-dependent cytosolic DNA sensing mediates innate immune recognition of immunogenic tumors. Immunity 41(5), 830-842 (2014).

-. First report showing that the STING pathway is essential for the induction of spontaneous T-cell responses in immunogenic tumors.

23 Corrales L, Glickman LH, McWhirter SM et al. Direct activation of STING in the tumor microenvironment leads to potent and systemic tumor regression and immunity. Cell Rep. 11(7), 1018-1030 (2015).

24 Wang $\mathrm{H}, \mathrm{Hu}$ S, Chen X et al. cGAS is essential for the antitumor effect of immune checkpoint blockade. Proc. Natl Acad. Sci. USA 114(7), 1637-1642 (2017).

25 Mikucki ME, Fisher DT, Matsuzaki J et al. Non-redundant requirement for CXCR3 signalling during tumoricidal T-cell trafficking across tumour vascular checkpoints. Nat. Commun. 67458 (2015).

26 Dong H, Strome SE, Salomao DR et al. Tumor-associated B7-H1 promotes T-cell apoptosis: a potential mechanism of immune evasion. Nat. Med. 8(8), 793-800 (2002). 
27 Hirano F, Kaneko K, Tamura H et al. Blockade of B7-H1 and PD-1 by monoclonal antibodies potentiates cancer therapeutic immunity. Cancer Res. 65(3), 1089-1096 (2005).

28 Chen L, Han X. Anti-PD-1/PD-L1 therapy of human cancer: past, present, and future. J. Clin. Invest. 125(9), 3384-3391 (2015).

29 Taube JM, Anders RA, Young GD et al. Colocalization of inflammatory response with B7-h1 expression in human melanocytic lesions supports an adaptive resistance mechanism of immune escape. Sci. Transl. Med. 4(127), 127ra137 (2012).

30 Taube JM, Klein A, Brahmer JR et al. Association of PD-1, PD-1 ligands, and other features of the tumor immune microenvironment with response to anti-PD-1 therapy. Clin. Cancer Res. 20(19), 5064-5074 (2014).

31 Gordon SR, Maute RL, Dulken BW et al. PD-1 expression by tumour-associated macrophages inhibits phagocytosis and tumour immunity. Nature 545(7655), 495-499 (2017).

32 Monney L, Sabatos CA, Gaglia JL et al. Th1-specific cell surface protein Tim-3 regulates macrophage activation and severity of an autoimmune disease. Nature 415(6871), 536-541 (2002).

33 Zhu C, Anderson AC, Schubart A et al. The Tim-3 ligand galectin-9 negatively regulates Thelper type 1 immunity. Nat. Immunol. 6(12), 1245-1252 (2005).

34 Ngiow SF, von Scheidt B, Akiba H, Yagita H, Teng MW, Smyth MJ. Anti-TIM3 antibody promotes T cell IFN-gamma-mediated antitumor immunity and suppresses established tumors. Cancer Res. 71(10), 3540-3551 (2011).

35 Sakuishi K, Apetoh L, Sullivan JM, Blazar BR, Kuchroo VK, Anderson AC. Targeting Tim-3 and PD-1 pathways to reverse T cell exhaustion and restore anti-tumor immunity. J. Exp. Med. 207(10), 2187-2194 (2010).

36 Leach DR, Krummel MF, Allison JP. Enhancement of antitumor immunity by CTLA-4 blockade. Science 271(5256), 1734-1736 (1996).

37 Hodi FS, Chesney J, Pavlick AC et al. Combined nivolumab and ipilimumab versus ipilimumab alone in patients with advanced melanoma: 2-year overall survival outcomes in a multicentre, randomised, controlled, Phase 2 trial. Lancet Oncol. 17(11), 1558-1568 (2016).

38 Larkin J, Chiarion-Sileni V, Gonzalez R et al. Combined nivolumab and ipilimumab or monotherapy in untreated melanoma. N. Engl. J. Med. 373(1), 23-34 (2015).

39 Triebel F, Jitsukawa S, Baixeras E et al. LAG-3, a novel lymphocyte activation gene closely related to CD4. J. Exp. Med. 171(5), 1393-1405 (1990).

40 Andrews LP, Marciscano AE, Drake CG, Vignali DA. LAG3 (CD223) as a cancer immunotherapy target. Immunol. Rev. 276(1), 80-96 (2017).

41 Woo SR, Turnis ME, Goldberg MV et al. Immune inhibitory molecules LAG-3 and PD-1 synergistically regulate T-cell function to promote tumoral immune escape. Cancer Res. 72(4), 917-927 (2012).

42 Munn DH, Mellor AL. IDO in the tumor microenvironment: inflammation, counter-regulation, and tolerance. Trends Immunol. 37(3), 193-207 (2016).

43 Uyttenhove C, Pilotte L, Theate I et al. Evidence for a tumoral immune resistance mechanism based on tryptophan degradation by indoleamine 2,3-dioxygenase. Nat. Med. 9(10), 1269-1274 (2003).

44 Fallarino F, Grohmann U, You S et al. The combined effects of tryptophan starvation and tryptophan catabolites down-regulate T cell receptor zeta-chain and induce a regulatory phenotype in naive T cells. J. Immunol. 176(11), 6752-6761 (2006).

45 Munn DH, Sharma MD, Hou D et al. Expression of indoleamine 2,3-dioxygenase by plasmacytoid dendritic cells in tumor-draining lymph nodes. J. Clin. Invest. 114(2), 280-290 (2004).

46 Speeckaert R, Vermaelen K, van Geel N et al. Indoleamine 2,3-dioxygenase, a new prognostic marker in sentinel lymph nodes of melanoma patients. Eur. J. Cancer 48(13), 2004-2011 (2012).

47 Ferdinande L, Decaestecker C, Verset L et al. Clinicopathological significance of indoleamine 2,3-dioxygenase 1 expression in colorectal cancer. Br. J. Cancer 106(1), 141-147 (2012).

48 Zhai L, Lauing KL, Chang AL et al. The role of IDO in brain tumor immunotherapy. J. Neurooncol. 123(3), 395-403 (2015).

49 Okamoto A, Nikaido T, Ochiai $\mathrm{K}$ et al. Indoleamine 2,3-dioxygenase serves as a marker of poor prognosis in gene expression profiles of serous ovarian cancer cells. Clin. Cancer Res. 11(16), 6030-6039 (2005).

50 Folgiero V, Goffredo BM, Filippini P et al. Indoleamine 2,3-dioxygenase 1 (IDO1) activity in leukemia blasts correlates with poor outcome in childhood acute myeloid leukemia. Oncotarget 5(8), 2052-2064 (2014).

51 Lemos H, Mohamed E, Huang L et al. STING promotes the growth of tumors characterized by low antigenicity via IDO activation. Cancer Res. 76(8), 2076-2081 (2016).

- Indicates that the stimulation of the STING pathway causes different responses depending on antigenicity in the tumor microenvironment.

52 Nishikawa H, Sakaguchi S. Regulatory T cells in cancer immunotherapy. Curr. Opin. Immunol. 27 1-7 (2014).

53 Teng MW, Ngiow SF, Ribas A, Smyth MJ. Classifying cancers based on T-cell infiltration and PD-L1. Cancer Res. 75(11), 2139-2145 (2015). 
54 Spranger S, Bao R, Gajewski TF. Melanoma-intrinsic beta-catenin signalling prevents anti-tumour immunity. Nature 523(7559), 231-235 (2015).

55 Peng D, Kryczek I, Nagarsheth $\mathrm{N}$ et al. Epigenetic silencing of TH1-type chemokines shapes tumour immunity and immunotherapy. Nature 527(7577), 249-253 (2015).

56 Galluzzi L, Buque A, Kepp O, Zitvogel L, Kroemer G. Immunological effects of conventional chemotherapy and targeted anticancer agents. Cancer Cell 28(6), 690-714 (2015).

57 Pfirschke C, Engblom C, Rickelt S et al. Immunogenic chemotherapy sensitizes tumors to checkpoint blockade therapy. Immunity 44(2), 343-354 (2016).

58 Langer CJ, Gadgeel SM, Borghaei H et al. Carboplatin and pemetrexed with or without pembrolizumab for advanced, non-squamous non-small-cell lung cancer: a randomised, Phase 2 cohort of the open-label KEYNOTE-021 study. Lancet Oncol. 17(11), 1497-1508 (2016).

59 Weber JS, Yang JC, Atkins MB, Disis ML. Toxicities of immunotherapy for the practitioner. J. Clin. Oncol. 33(18), 2092-2099 (2015).

60 Yang L, Yu H, Dong S, Zhong Y, Hu S. Recognizing and managing on toxicities in cancer immunotherapy. Tumour Biol. 39(3), $1010428317694542(2017)$

61 Trombetta ES, Mellman I. Cell biology of antigen processing in vitro and in vivo. Annu Rev Immunol. 23 975-1028 (2005).

62 Harding CV, Collins DS, Kanagawa O, Unanue ER. Liposome-encapsulated antigens engender lysosomal processing for class II MHC presentation and cytosolic processing for class I presentation. J. Immunol. 147(9), 2860-2863 (1991).

63 Nakamura T, Moriguchi R, Kogure K, Shastri N, Harashima H. Efficient MHC class I presentation by controlled intracellular trafficking of antigens in octaarginine-modified liposomes. Mol. Ther. 16(8), 1507-1514 (2008).

64 Nakamura T, Ono K, Suzuki Y, Moriguchi R, Kogure K, Harashima H. Octaarginine-modified liposomes enhance cross-presentation by promoting the C-terminal trimming of antigen peptide. Mol. Pharm. 11(8), 2787-2795 (2014).

65 Rosalia RA, Silva AL, Camps M et al. Efficient ex vivo induction of $\mathrm{T}$ cells with potent anti-tumor activity by protein antigen encapsulated in nanoparticles. Cancer Immunol. Immunother. 62(7), 1161-1173 (2013).

66 Keller S, Wilson JT, Patilea GI, Kern HB, Convertine AJ, Stayton PS. Neutral polymer micelle carriers with pH-responsive, endosome-releasing activity modulate antigen trafficking to enhance CD8(+) T cell responses. J. Control. Rel. 191 24-33 (2014).

67 Zang X, Zhao X, Hu H, Qiao M, Deng Y, Chen D. Nanoparticles for tumor immunotherapy. Eur. J. Pharm. Biopharm. 115 243-256 (2017).

68 Brubaker SW, Bonham KS, Zanoni I, Kagan JC. Innate immune pattern recognition: a cell biological perspective. Annu. Rev. Immunol. 33 257-290 (2015).

69 Junt T, Barchet W. Translating nucleic acid-sensing pathways into therapies. Nat. Rev. Immunol. 15(9), 529-544 (2015).

70 Hafner AM, Corthesy B, Merkle HP. Particulate formulations for the delivery of poly(I:C) as vaccine adjuvant. Adv. Drug Deliv. Rev. 65(10), 1386-1399 (2013).

71 Malyala P, O’Hagan DT, Singh M. Enhancing the therapeutic efficacy of CpG oligonucleotides using biodegradable microparticles. Adv. Drug Deliv. Rev. 61(3), 218-225 (2009).

72 Wilson KD, de Jong SD, Tam YK. Lipid-based delivery of CpG oligonucleotides enhances immunotherapeutic efficacy. Adv. Drug Deliv. Rev. 61(3), 233-242 (2009).

73 Nakamura T, Moriguchi R, Kogure K, Harashima H. Incorporation of polyinosine-polycytidylic acid enhances cytotoxic T cell activity and antitumor effects by octaarginine-modified liposomes encapsulating antigen, but not by octaarginine-modified antigen complex. Int J. Pharm. 441(1-2), 476-481 (2013).

74 Matsumoto M, Tatematsu M, Nishikawa F et al. Defined TLR3-specific adjuvant that induces NK and CTL activation without significant cytokine production in vivo. Nat. Commun. 66280 (2015).

75 Takeda Y, Kataoka K, Yamagishi J, Ogawa S, Seya T, Matsumoto M. A TLR3-specific adjuvant relieves innate resistance to PD-L1 blockade without cytokine toxicity in tumor vaccine immunotherapy. Cell. Rep. 19(9), 1874-1887 (2017).

76 Temizoz B, Kuroda E, Ishii KJ. Vaccine adjuvants as potential cancer immunotherapeutics. Int. Immunol. 28(7), 329-338 (2016).

77 Kobiyama K, Aoshi T, Narita H et al. Nonagonistic dectin-1 ligand transforms CpG into a multitask nanoparticulate TLR9 agonist. Proc. Natl Acad. Sci. USA 111(8), 3086-3091 (2014).

78 Miyabe H, Hyodo M, Nakamura T, Sato Y, Hayakawa Y, Harashima H. A new adjuvant delivery system 'cyclic di-GMP/YSK05 liposome' for cancer immunotherapy. J. Control. Rel. 184 20-27 (2014).

-• First report showing that STING agonist loaded nanoparticles are a potent adjuvant system for cancer immunotherapy.

79 Parvatiyar K, Zhang Z, Teles RM et al. The helicase DDX41 recognizes the bacterial secondary messengers cyclic di-GMP and cyclic di-AMP to activate a type I interferon immune response. Nat. Immunol. 13(12), 1155-1161 (2012).

80 Sato Y, Hatakeyama H, Sakurai Y, Hyodo M, Akita H, Harashima H. A pH-sensitive cationic lipid facilitates the delivery of liposomal siRNA and gene silencing activity in vitro and in vivo. J. Control. Rel. 163(3), 267-276 (2012). 
81 Ahmad M, Rees RC, Ali SA. Escape from immunotherapy: possible mechanisms that influence tumor regression/progression. Cancer Immunol. Immunother. 53(10), 844-854 (2004).

82 Nakamura T, Miyabe H, Hyodo M, Sato Y, Hayakawa Y, Harashima H. Liposomes loaded with a STING pathway ligand, cyclic di-GMP, enhance cancer immunotherapy against metastatic melanoma. J. Control. Rel. 216 149-157 (2015).

83 Hanson MC, Crespo MP, Abraham W et al. Nanoparticulate STING agonists are potent lymph node-targeted vaccine adjuvants. J. Clin. Invest. 125(6), 2532-2546 (2015).

84 Luo M, Wang H, Wang Z et al. A STING-activating nanovaccine for cancer immunotherapy. Nat. Nanotechnol.12 (7), 648-654 (2017).

85 Redelman-Sidi G, Glickman MS, Bochner BH. The mechanism of action of BCG therapy for bladder cancer - a current perspective. Nat. Rev. Urol. 11(3), 153-162 (2014).

86 Lamm DL, van der Meijden PM, Morales A et al. Incidence and treatment of complications of bacillus Calmette-Guerin intravesical therapy in superficial bladder cancer. J. Urol. 147(3), 596-600 (1992).

87 Azuma I, Seya T. Development of immunoadjuvants for immunotherapy of cancer. Int. Immunopharmacol. 1(7), 1249-1259 (2001).

88 Uenishi Y, Kawabe K, Nomura T, Nakai M, Sunagawa M. Morphological study on Mycobacterium bovis BCG Tokyo 172 cell wall skeleton (SMP-105). J. Microbiol. Methods 77(2), 139-144 (2009).

89 Nakamura T, Fukiage M, Higuchi M et al. Nanoparticulation of BCG-CWS for application to bladder cancer therapy. J. Control. Rel. $17644-53$ (2014).

- First report of the successful encapsulation of Bacillus Calmette-Guerin cell wall skeleton into lipid nanoparticles.

90 Nakamura T, Fukiage M, Suzuki Y et al. Mechanism responsible for the antitumor effect of BCG-CWS using the LEEL method in a mouse bladder cancer model. J. Control. Rel. 196 161-167 (2014).

91 Trevaskis NL, Kaminskas LM, Porter CJ. From sewer to saviour - targeting the lymphatic system to promote drug exposure and activity. Nat. Rev. Drug Discov. 14(11), 781-803 (2015).

92 Reddy ST, van der Vlies AJ, Simeoni E et al. Exploiting lymphatic transport and complement activation in nanoparticle vaccines. Nat. Biotechnol. 25(10), 1159-1164 (2007).

93 Thomas SN, Vokali E, Lund AW, Hubbell JA, Swartz MA. Targeting the tumor-draining lymph node with adjuvanted nanoparticles reshapes the anti-tumor immune response. Biomaterials 35(2), 814-824 (2014).

94 Liu H, Moynihan KD, Zheng Y et al. Structure-based programming of lymph-node targeting in molecular vaccines. Nature 507(7493), 519-522 (2014).

95 Kuai R, Ochyl LJ, Bahjat KS, Schwendeman A, Moon JJ. Designer vaccine nanodiscs for personalized cancer immunotherapy. Nat Mater 16(4), 489-496 (2017).

96 Blank CU, Haanen JB, Ribas A, Schumacher TN. CANCER IMMUNOLOGY. The 'cancer immunogram'. Science 352(6286), 658-660 (2016).

97 Warashina S, Nakamura T, Sato Y et al. A lipid nanoparticle for the efficient delivery of siRNA to dendritic cells. J. Control. Rel. 225 183-191 (2016).

98 Nakamura T, Kuroi M, Fujiwara Y, Warashina S, Sato Y, Harashima H. Small-sized, stable lipid nanoparticle for the efficient delivery of siRNA to human immune cell lines. Sci. Rep. 637849 (2016). 



\title{
Therapeutic Delivery
}

\section{Nanomedicine for cancer diagnosis and therapy: advancement, success and structure-activity relationship}

\author{
Ketki Bhise1, Samaresh Sau¹, Hashem Alsaab1, Sushil Kumar Kashaw ${ }^{1,2}$, Rakesh Kumar \\ Tekade $^{3}$ \& Arun K lyer*,1,4 \\ ${ }^{1}$ Use-inspired Biomaterials \& Integrated Nano Delivery (U-BiND) Systems Laboratory, Department of Pharmaceutical Sciences, \\ Eugene Applebaum College of Pharmacy \& Health Sciences, Wayne State University, 259 Mack Ave, Room 3601, Detroit, Ml \\ 48201, USA \\ ${ }^{2}$ Department of Pharmaceutical Sciences, Dr. Harisingh Gour University (a Central University), Sagar, MP, India \\ ${ }^{3}$ National Institute of Pharmaceutical Education \& Research (NIPER), Ahmedabad, Gujarat, India \\ ${ }^{4}$ Molecular Imaging Program, Barbara Ann Karmanos Cancer Institute, Wayne State University School of Medicine, Detroit, MI, \\ USA \\ * Author for correspondence: Tel.: +1 313577 5875; Fax: +1 313577 2033; arun.iyer@wayne.edu
}

Multifunctional nanoparticles (NPs), composed of organic and inorganic materials, have been explored as promising drug-delivery vehicles for cancer diagnosis and therapy. The success of nanosystems has been attributed to its smaller size, biocompatibility, selective tumor accumulation and reduced toxicity. The relationship among numbers of molecules in payload, NP diameter and encapsulation efficacy have crucial role in clinical translation. Advancement of bioengineering, and systematic fine-tuning of functional components to NPs have diversified their optical and theranostic properties. In this review, we summarize wide varieties of NPs, such as ultrasmall polymer-lipid hybrid NPs, dendrimers, liposomes, quantum dots, carbon nanotubes, gold NPs and iron oxide NPs. We also discuss their tumor targetability, tissue penetration, pharmacokinetics, and therapeutic and diagnostic properties.

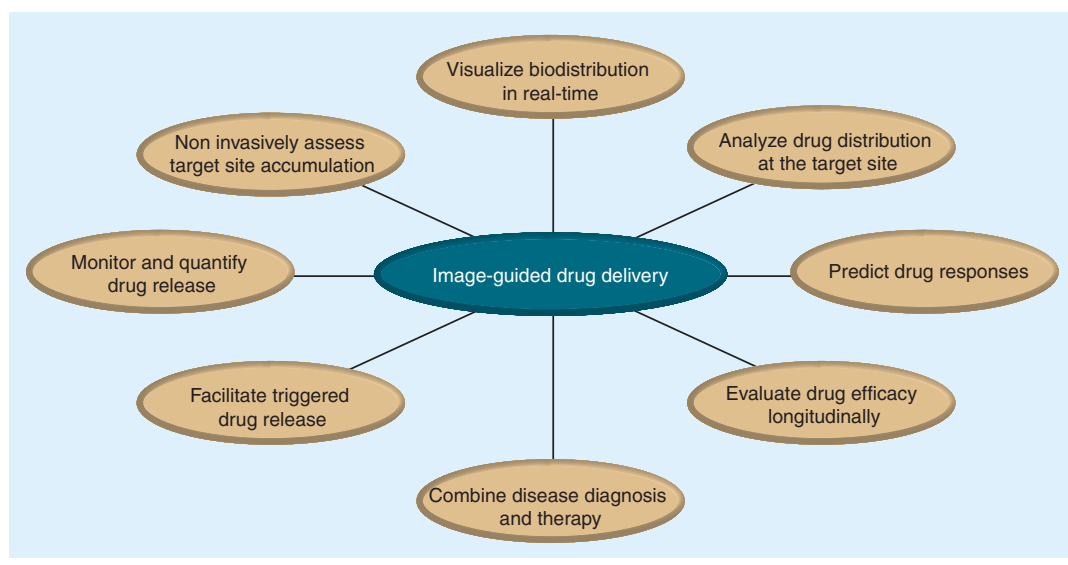

First draft submitted: 23 May 2017; Accepted for publication: 1 September 2017; Published online:

24 October 2017

Keywords: inorganic and organic nanoparticles • polymer-lipid hybrid nanosystem • theranostic nanoparticles • tumor stroma penetration • ultrasmall nanoparticle

'Theranostics,' a recent entrant in the vast field of medical nanotechnology, is lately achieving remarkable heights in patient healthcare systems. It combines the advantages of therapy and diagnosis, simultaneously delivering agents for both on a single platform. Taking advantage of their small size and large surface area, nanoparticles (NPs) play a pivotal role in realization of multimodal theranostics. The large surface area and accessibility to manipulate surface functional groups make NPs an ideal platform for theranostics. Separate investigations on therapeutic and 


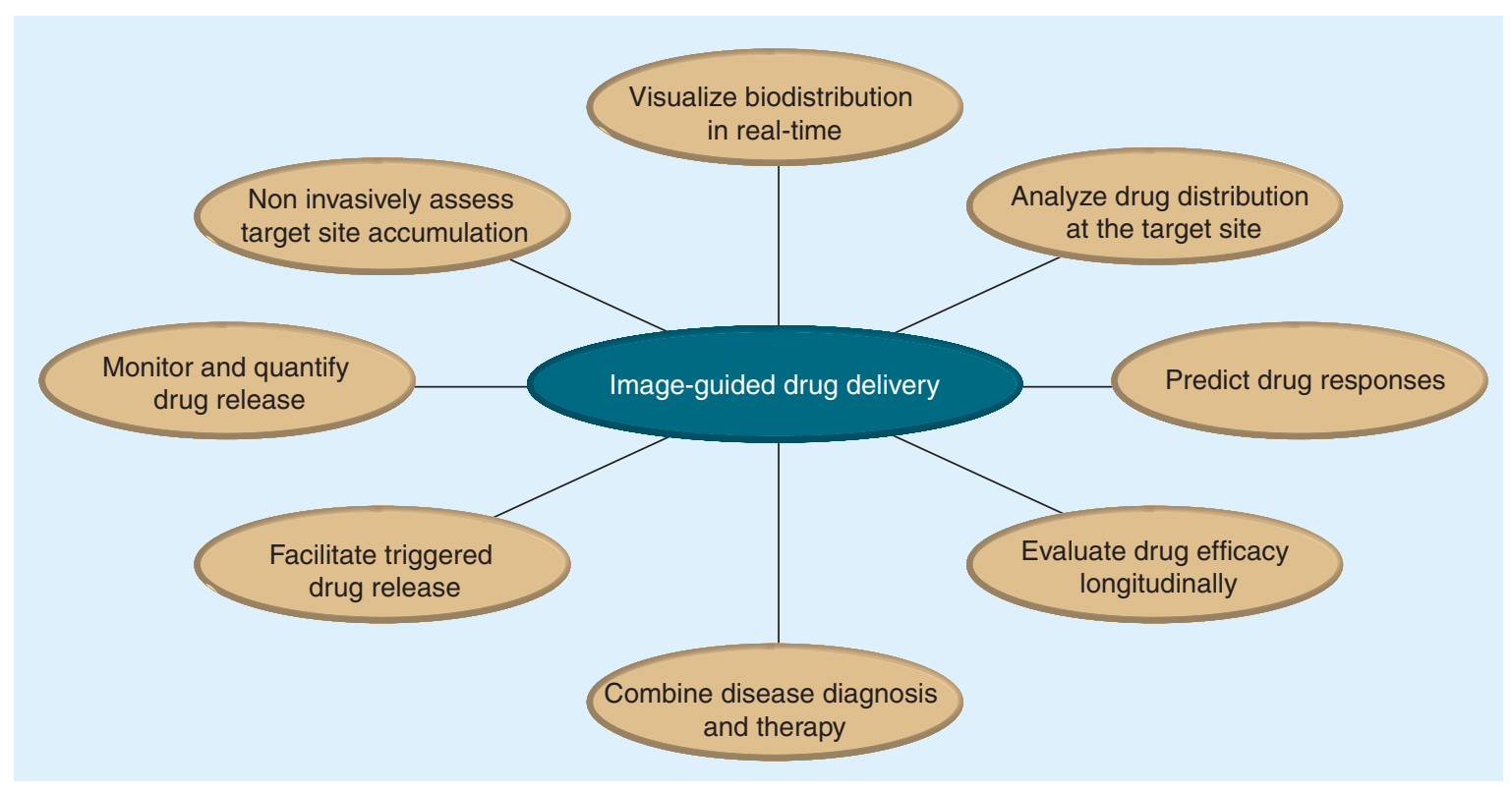

Figure 1. Advantages of nanotheranostics in cancer domain. Reproduced with permission from [14] (c) 2010.

nanomedical diagnostics have called for a relatively unmet need to combine both these aspects and arrive at a multifunctional platform delivering both in the form of theranostic NPs. Particularly in cancer, where such systems have been greatly studied upon, they improve the ability of disease prognosis and therapy.

Cancer is the second leading cause of death in the USA. As per a recent study, about 1,688,780 new cases of cancer are estimated to be detected in 2017, with an expected mortality of 600,920 [1]. Most of the conventional cancer treatments, such as small molecule-based drugs, and combination drug therapy to target tumors at different stages of growth, face challenges $[2,3]$ to differentiate between cancerous and noncancerous cells [4]. Heterogeneity in tumor cells and delay in treatment where the tumor has invariably metastasized, are some of the factors responsible for high mortality rates. It is thus imperative for early diagnosis of tumors, so that therapy can be efficiently targeted [5]. Theranostics offer a novel platform to perform imaging simultaneously alongside treatment, so that the tumor growth can be visualized and treated at various stages of development. Many NPs in development incorporate imaging agents. The task is to combine therapeutic agents to them and arrive at a theranostic module.

For an efficient response, it is necessary for NPs to accumulate at the site of action in sufficient amount. This can be achieved by active and passive targeting. Passive targeting takes the advantage of enhanced permeability and retention (EPR) effect [6-11]. Active targeting considers unique biomarkers on surface of tumor cells, each varying from tumor type-to-type [12]. This heterogeneity in biomarker population allows researchers to design ligands targeting the biomarkers [13]. Such NPs can be mounted with imaging agents and other therapeutic functionalities onto a single platform. Care must be taken for the toxicity of components of the multimodal system, their stability and degradation by body enzymes and immune response components.

With NPs for therapy and imaging each foraying into clinical trials, there is a push for early developmental research in theranostics as well. Some of the advantages of nanotheranostics are summarized in Figure 1 [14]. NPs are primarily composed of proteins, peptides, lipids, polymers, metals and their oxides. While each of these can be used for theranostic purpose, the most common NPs include dendrimers, micelles, liposomes, carbon nanotubes (CNTs), quantum dots (QDs), iron oxide NPs, gold nanoparticles (AuNPs) and silica NPs. Optical imaging, MRI, ultrasound, computed tomography (CT), positron emission tomography (PET), single-photon emission computed tomography (SPECT) are some of the imaging modalities [15-17]. Modern research is built upon developing multifunctional nanocarriers that include lipid-, thiol-, PEG-modified polymeric nanocarriers functionalized with therapeutic, targeting and imaging moieties $[7,18]$. This review article will briefly summarize the latest developments in nanoparticulate theranostic research for cancer.

Figure 2 shows relationship between number of molecules in payload and NP diameter [19]. The encapsulation 
Figure 2. Relationship between number of drug molecules and nanoparticle diameter. Reproduced with permission from [19] (c) 2012.

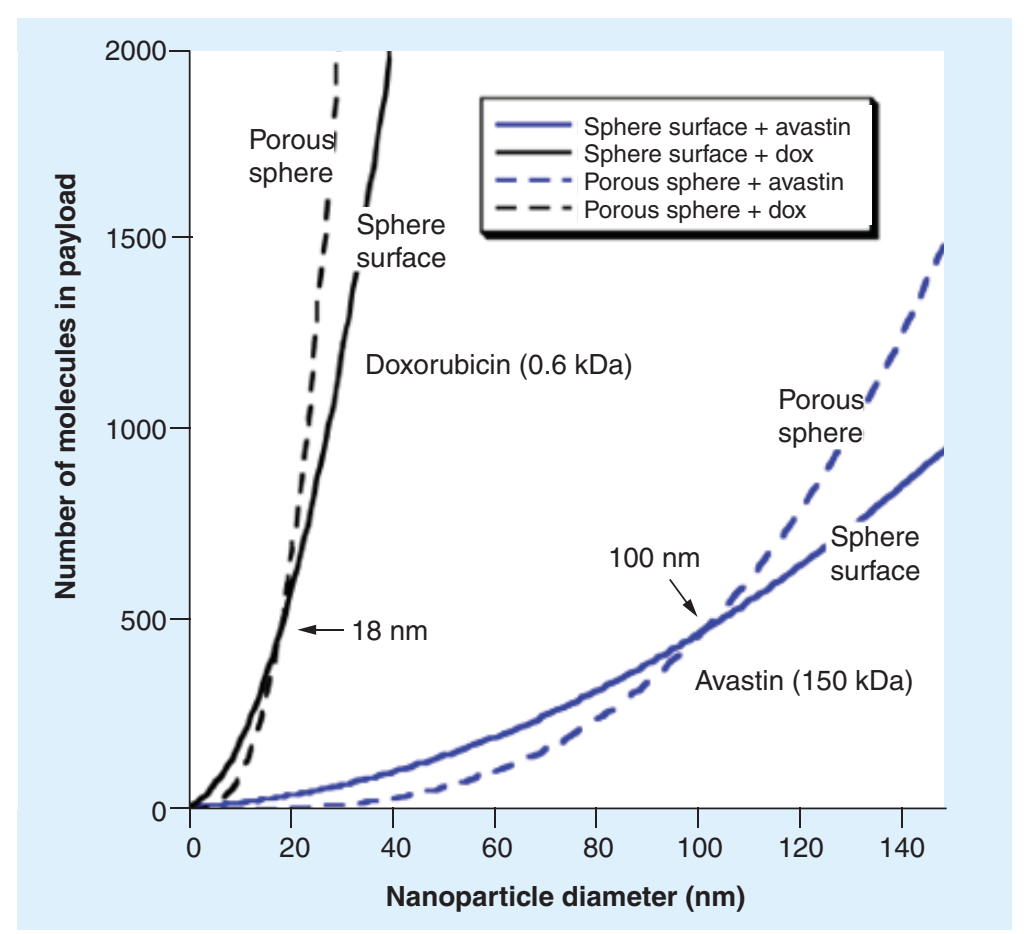

of payload depends on the volume of the hollow interior of the NP: the lesser the volume, the lesser will be the encapsulation of the payload. However, within the size cutoff of the NP, as it approaches the molecular size of the payload, there will be more surface area exposed for accommodation of the drug molecules than in the interior volume of the NP. The number of molecules accommodated will depend on the molecular weight of the NP, as reflected by small-molecule doxorubicin (Dox) and large antibody Avastin.

\section{Classes of theranostic nanocarriers}

\section{Ultrasmall lipid polymer hybrid NP}

This is a novel class of ultrasmall (sub $25 \mathrm{~nm}$ ) lipid-polymer hybrid NPs that have been developed for the purpose of deep tumor tissue penetration [20]. These hybrid NPs have been developed with help of nanoprecipitation techniques, where a polymer such as poly(lactic-co-glycolic acid) resides as core-shell of NP and a PEGylated lipid such as 1,2-distearoyl-sn-glycero-3-phosphoethanolamine-N-(methoxy[polyethylene glycol]-2000) is used to coat on the surface of the poly(lactic-co-glycolic acid), so that polymer-encapsulated drug, gene and dye can be stabilized [21]. As these types of NPs are bestowed with ultrasmall size, they have the ability to penetrate tumor stroma, tumor stem cell, and are also being considered to be utilized for brain tumor drug delivery [20]. Ultimately, with biocompatibility and ultrasmall size range, the hybrid version of NPs helps to boost the efficacy of nanoplatform by means of improving the therapeutic profile of drug payloads for cancer therapy and diagnosis applications.

\section{Dendrimers}

Dendrimers are hyperbranched polymeric nanostructures whose branching can be controlled by their generation number. Drugs and contrast agent can be incorporated in the branched architecture with high payload, while surface functionalities are amenable to modifications by different functional groups [22]. Polyamidoamine (PAMAM) dendrimers have been used as a carrier for methotrexate, with folate-targeting ligand and fluorescein as the fluorophore. Intravenously injected formulation showed uptake by tumor cells as detected by the fluorescent signal of the fluorophore [23]. Purified recombinant FGF-1 was chemically conjugated to G5 PAMAM dendrimer. The dendrimer was further conjugated to fluorescein isothiocyanate (FITC) and uptake of the conjugate was tested on cell lines expressing receptor for FGF by confocal microscopy and flow cytometry analysis. The imaging showed accumulation of the dendrimers in nucleus of tumor cells, thus demonstrating nuclear uptake of therapeutic agent [24]. Photolytic cleavage of Dox from folate-targeted G5 PAMAM dendrimer was demonstrated where Dox was released via a photochemical mechanism [25]. Therapeutic PEGylated polyester dendrimers loaded with Dox were constructed 
and imaged by radiolabeled iodine isotope ${ }^{125} \mathrm{I}$ for assessing biodistribution in vivo [26]. Dendritic PET-imaging nanoprobe was developed for the noninvasive imaging of angiogenesis in tumor vasculature. The nanoprobes were radiolabeled with ${ }^{125} \mathrm{I}$ and ${ }^{76} \mathrm{Br}$ where the latter showed good accumulation in tumor cells [27]. Synthesis of multifunctional G5 PAMAM dendrimer entrapping AuNPs was undertaken. The dendrimers were functionalized with $\alpha$-tocopherol succinate and labeled with fluorescein isothiocyanate for CT imaging. The nanoarchitecture was surface-functionalized with folic acid and imaged in cancer cells in vitro and tumor xenograft in vivo [28]. G5 polypropylenimine dendrimer was synthesized, encapsulating substituted silicon naphthalocyanine into its hydrophobic core, followed by surface decoration with PEG. Near-infrared (NIR) dye was employed as the imaging agent in this hybrid system, which was finally coupled with photodynamic-photothermal therapy (PDT-PTT) [29]. G5-PAMAM dendrimers, conjugated with fluorescein isothiocyanate, PEGylated arginine-glycine-aspartic acid (RGD) peptide, PEGylated $\alpha$-tocopheryl succinate and coupled with AuNP were synthesized to efficiently target and image cancer cells. The cancer cells were subjected to CT imaging in vitro [30].

\section{Liposomes}

Liposomes are vesicular structures that have the propensity to load both hydrophilic and hydrophobic molecules, because of which they are amenable to loading of therapeutic drugs and imaging agents. Studies involving liposomes loaded with stable PET tracers like ${ }^{64} \mathrm{Cu}$ were conducted to evaluate the diagnostic potential of the tracer in animals at acceptable doses [31]. Taking advantage of the possibility to modulate liposomal loading with a variety of molecules that are not only restricted to drugs or imaging agents, research with novel targeting strategies like PTT has gained momentum. Pang et al. have demonstrated the construction of PEGylated folic acid-conjugated liposomes encapsulating indocyanine green, an NIR dye along with rapamycin as the anticancer drug. Indocyanine green is known to have high NIR absorption with implications in PTT [32]. Lanthanide-doped luminescent upconverting NPs are those that can convert low-energy NIR excitation light to high energy. Because of the large spectrum of wavelength that they can cover, such NPs have wide imaging applications. Lanthanides and Dox were incorporated into the aqueous core of liposomes, serving as a platform for therapeutic and imaging functionalities [33]. ${ }^{111} \mathrm{In}$ radiolabeled, $\mathrm{pH}$-responsive liposomes showed prolonged circulation in bloodstream, high radiolabeling efficacy and little toxicity to major organs [34]. Multifunctional liposomes tagged with targeting ligand and possessing therapeutic and imaging potential are currently under active research. Immunoliposomes with

${ }^{111}$ In as the imaging agent and labeled with human single-chain antibody fragment targeting mesothelioma were developed by Iyer et al. The developed liposomes demonstrated preferentially high accumulation in target tumor tissues as compared with other vital organs [15]. Liposomes decorated with RGD-TPGS for targeting brain tumor, and encapsulating docetaxel (DTX) for therapy and QDs for imaging, have been developed. RGD sequence is a known recognition motif for integrin $\alpha \nu \beta 3$ that is overexpressed in actively proliferating tumor endothelial cells [35]. The same group has also reported transferrin-conjugated liposomes targeting brain tumor, and encapsulating QDs for imaging and DTX for therapy purpose [36]. In an application of PDT, a photosensitizer hexylamine-conjugated chlorin e6 along with a lipophilic NIR dye 1,1'-dioctadecyl-3,3,30,3'-tetramethylindotricarbocyanine iodide were simultaneously encapsulated in PEGylated liposome, where the hexylamine-conjugated chlorin e6 was quenched by $1,1^{\prime}$-dioctadecyl-3,3,30,3'-tetramethylindotricarbocyanine iodide by utilizing fluorescence resonance energy transfer (FRET). Such novel systems have implications in cancer phototherapy [37]. Thermally sensitive liposomes enable imaging as well as release of cargo under local heating conditions. In this regard, liposomes coated with gold were synthesized and studied for imaging and drug release. The formed liposomes showed strong absorption in NIR region and drug release in tumor cells [38].

\section{Polymeric micelles}

Polymeric micelles are amenable to surface modification because of functionalization of surface functional groups [3941]. Moreover, they improve drug solubility and loading [8]. The versatility of these self-assembled, stable structures is evident from the vast number of studies utilizing micelles for combination of a range of therapy and imaging agents $[8,42]$. Green synthesis of theranostic micelles has been reported, wherein block amphiphilic polymers were used in self-assembly of dual-purpose micelles. Pluronic F 127 and polypeptide modified with palmitic acid were incorporated with superparamagnetic iron oxide nanoparticles (SPIONs) and Dox as the imaging and chemotherapeutic agents, respectively. Such micelles loaded with SPIONs facilitate in vivo MRI contrast imaging. The magnetic NPs assemble on the shell of the micelles. The propensity to self-assemble into complex structures without the need of toxic chemical reactions is a major advantage of micellar structures $[43,44]$. On the other 
hand, a well-known covalent chemistry has been explored to conjugate Dox to the amphiphilic arm of block copolymer via a $\mathrm{pH}$ cleavable hydrazone linkage, facilitating release in tumor acidic microenvironment. The complex structure thus formed, consisting of the aforementioned 'arm' conjugated to cyclic RGD peptide and macrocyclic chelators was tested in tumor-bearing mice model. Macrocyclic chelators were employed for ${ }^{64} \mathrm{Cu}$ labeling and PET imaging [45]. SPECT is a nuclear imaging technique that makes use of gamma rays and provides high-resolution images with good sensitivity, selectivity and ease of handling the instrumentation [46]. SPECT imaging in animal models shows rapid internalization of tumor-targeting human single-chain antibody fragment tagged with ${ }^{99 \mathrm{~m}} \mathrm{Tc}$ within $1 \mathrm{~h}$ of injection $[16,17]$. Apart from SPIONs and PET imaging modalities, NIR dyes have also been used along with chemotherapeutic agents to serve the 'dual-purpose' strategy. With this regard, carbocyanine dyes were loaded into copolymer micelles and studied for tumor retention in vivo [47]. In yet another modality, TPGS alternately conjugated with transferrin and gold nanoclusters, and loaded with DTX was used to form micelles by the solvent-casting method. Such particles offer targeting to specific tumor types, along with performing theranostic application [48]. QDs have also been utilized as imaging agent along with phospholipid-based polymeric micelles. QDs and Dox were coloaded in the hydrophobic core of the micelles and characterized for optical spectroscopy, wherein the incorporation of Dox also serves as an advantage in imaging, apart from QDs [49]. PTT and PDT are novel targeting strategies whose efficiency can be enhanced by coupling with dual-imaging modality. Dual imaging recruited photoacoustic (PA)/NIR fluorescent imaging, creating a bright contrast to locate the exact tumor localization in vivo. Cypate, a cyanine dye was utilized for the purpose of imaging [50]. Chlorin e6 is a photosensitizer that can be activated by the NIR dye, making it useful in NIR fluorescent. Its application in PA imaging is also studied. Taking advantage of the dual-imaging modality, as well as abundance of enzyme hyaluronidase in tumor cells, hyaluronic acid (HA)-based micelles were developed that specifically targeted CD44 receptors $[7,51,52]$. Gadolinium contrast agents have been studied in $T_{1}$ MRI but are limited by high nephrotoxicity that limits their use in vivo. Nontoxic, electronically similar contrast agents like manganese have replaced gadolinium in $T_{1}$ MRI. Phospholipid-based micelles were developed, carrying drug and gene payload and targeting lung cancer cells [53]. Prodrugs are moieties that are inactive outside the body and are activated once cleaved inside the body. Prodrug approach essentially makes the drug therapeutically available only after action of specific enzymes or processes in the human body. One such prodrug approach has been tested in theranostic micelles for overcoming drug resistance. Gemcitabine reduction-sensitive polymeric prodrug containing IR820 dye for NIR imaging was synthesized by novel 'click' chemistry. Reduction-sensitive disulfide bond bridged between gemcitabine and the polymer backbone. The complex structure formed self-assembled micelles and showed enhanced uptake in vivo as confirmed by NIR imaging [54]. Figure 3 shows ex vivo imaging of various organs to show uptake of control, targeted and nontargeted DTX-loaded theranostic micelles [48].

\section{Carbon nanotubes}

Carbon exhibits various allotropic forms, each one having distinctive property and applications in varied fields. CNT is an allotrope of carbon, with a wide range of applications [55]. Because of their large surface area, they have been under study as imaging and therapy agents. Imaging is done by means of thermal ablation, and the therapeutic moieties include gene and drug. CNTs are efficiently taken up by the cells as well. CNTs broadly fall into one of the two main classes: single- and multi-walled. Single-walled CNTs are under active research for their numerous imaging advantages, like ability to show Raman scattering, strong PA signal, high MR contrast and stable NIR photoluminescence [56]. Surface modifications are necessary to avoid aggregation and toxicity of the NPs when injected in vivo. Single-walled nanotubes are capable of absorbing NIR light. Advances on NIR irradiation of single-walled nanotubes have been reported recently. Researchers have reported mediation of cancer cell killing by photodynamic effect coupled with single-walled nanotubes [57]. For formulation of long-circulating NPs, PEGylation is an effective technique to evade uptake by the mononuclear phagocytic system. However, the PEGylated NPs are unselectively taken up by anti-PEG IgM antibodies and destined for clearance $[58,59]$. In order to prolong circulation, albumin was used in place of PEG and encapsulated with Chlorin 6, a photosensitizer with strong near fluorescence and effective display of photodynamic and photothermal activity. Evans blue is a hydrophilic and nontoxic stain and possesses high affinity for albumin. Covalent conjugation on surface of single-walled CNTs may disrupt its architecture. The stain was noncovalently adsorbed on single-walled CNTs, followed by loading of the albumin-chlorin 6 complex. The combination of such a hybrid system possesses advantages of both PA and fluorescence imaging, allowing one to use PDT-PTT in combination on a single platform [60]. Another modality wherein PTT has been used in conjunction with single-walled CNTs was described by Liang et al. for targeting 


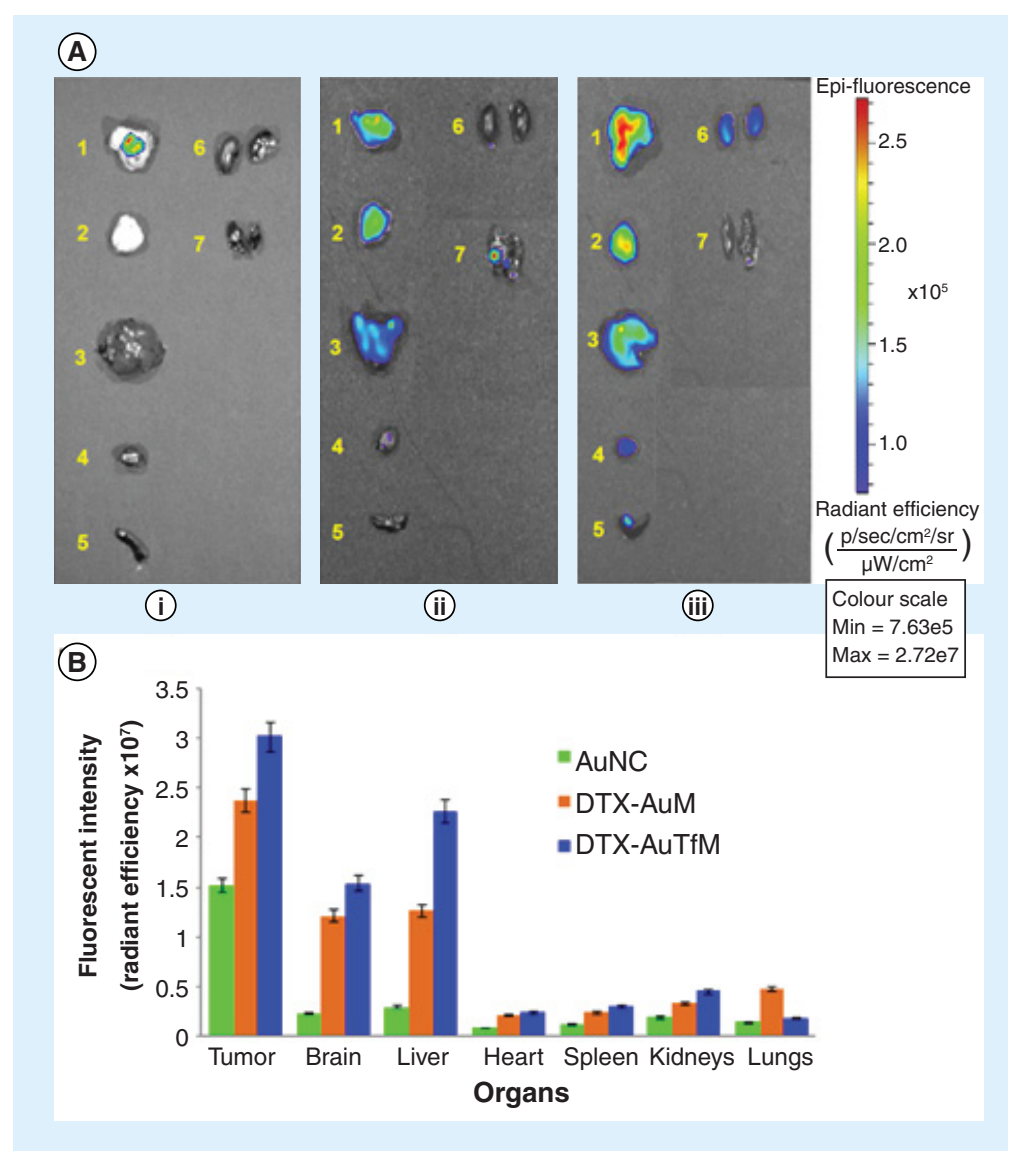

Figure 3. Fluorescence exhibited in different organs by ultrabright gold clusters, docetaxel-loaded TPGS gold theranostic micelles and docetaxel-loaded TPGS gold-transferrin theranostic micelles. Reproduced with permission from [48] (c) 2015.

breast cancer. Infrared-fluorescent active cyanine dye, Cy5.5 was conjugated to the nanotube. Tumor was traced by NIR imaging and then PTT was exploited by exposure to laser. Both the in vitro and in vivo results presented promising tumor cell inhibition [61]. Highly water-soluble, stable polydopamine-functionalized single-walled CNTs were developed that offered additional loading of $\mathrm{Mn}^{2+}$ for effective T1 and T2 MR contrast. The polydopamine shell also allowed coating with ${ }^{131} \mathrm{I}$, allowing multiple therapies within a single module [62]. Single-walled CNTs armed with the ability to convert ultrashort pulse microwave signals to strong thermoacoustic shock wave, killing cancer cells were developed by Wen $e t$ al. Deep-seated, orthotropic liver model was used to study the mitochondrial damage ensuing from the shock wave. The study demonstrated a high tumor cell killing of $77.5 \%$, suggested by apoptosis caused by damage to mitochondria [63]. Wang et al. demonstrated efficient in vitro and in vivo activity of DTX noncovalently conjugated to single-walled CNTs, further modified with Asn-Gly-Arg peptide for targeting angiogenetic factors [64]. In another study, multiwalled CNTs were functionalized with folate and iron, loaded with Dox. Iron serves to localize the NPs under the influence of external magnetic field, whereas folate ligand targets the NP to folate receptor expressed on the surface of HeLa cells [65]. With the above case studies, it has been briefly reviewed that $\mathrm{CNT}$ s possess the propensity to engage with various cancer therapies, ranging from PDT/PTT to microwave assisted to the conventional chemotherapy. The versatility of such systems increases manifold with the inherent imaging ability of CNTs.

\section{Iron oxide NPs}

A major challenge pertaining to iron oxide NPs is the tendency to agglomerate. In order to reduce this, scientists have achieved coating of iron oxide NPs [66] with materials compatible with MRI. While iron oxide NPs do not achieve therapy per se, they need to be modified with functional groups complexed with drug, or as an enhancement to this, surface decorated with active targeting moieties. Dual-targeting iron oxide NPs showed promising contrast MRI imaging to hepatocellular carcinoma (HCC) [62]. Coating with lipids or polymers increases the particle size of the NPs, while endowing them with increased stability in body fluids. Unterweger $e$ t al. synthesized polymerdrug complex with HA and cisplatin that selectively target CD44 receptors and utilize SPIONs as imaging agent. 
The hydroxyl group of dextran was used to conjugate HA over the surface of SPIONs [67]. SPIONs without dextran modification of HA have also been developed [68]. Aptamers are being studied as attractive targeting agents to increase specificity in cancer cell types. In a recent study, SPIONs synthesized by the microemulsion method were surface coated with gold to decrease cytotoxicity associated with SPIONs and to combine with the advantage of gold photothermal effect. The resulting NPs were conjugated with thiol-modified MUC-1 aptamers, with an overall particle size of $19 \mathrm{~nm}$. The theranostic NPs showed effective in vitro activity in colon cancer cell lines [69]. Targetability of SPIONs was also enhanced by coating the NPs with anti-EGFR monoclonal antibody specific to EGFR receptors overexpressed in lung cancer [70]. Iron oxide NPs are also being studied for DNA and siRNA delivery, which remains a challenge in clinical settings. Nucleic acids being negatively charged, a material with cationic character suitably condenses them with minimum degradation [71]. Iron oxide NPs coated with cationic lipids with an ability to condense nucleic acids were developed [72]. The NPs exhibited slightly higher particle size than expected out of SPIONs, probably due to coating with cationic lipids. Apart from coating with polymers, gold and lipids, SPIONs were also surface functionalized with PAMAM dendrimers, strengthening the confidence in SPIONs for multiple types of surface functionalization. Luong et al. surface-modified SPIONs with folate-functionalized PAMAM dendrimers loaded with drug curcumin difluorinated [CDF] (a synthetic curcumin analogue), which showed enhanced anticancer activity in vitro [73]. SPIONs modified with folic acid alone were also synthesized [74]. Exhaustive chemistry was utilized in development of iron oxide NPs with conjugation of drug and monoclonal antibody. The drug, gemcitabine, was conjugated on the surface of activated iron oxide NPs via 1ethyl-3-(3-dimethylaminopropyl)carbodiimide/N-Hydroxysuccinimide (EDC/NHS) coupling. Furthermore, in order to target CD47 receptor overexpressed on pancreatic cancer cells, anti-CD47 antibody was immobilized on the surface of iron oxide NPs [75]. The literature is full with iron oxide NPs for theranostic purpose. Our aim was to briefly highlight examples representative of categories of surface modifications of iron oxide NPs.

\section{Quantum dots}

QDs have garnered attention owing to remarkable imaging property, however, their use is limited by cellular toxicity. Several attempts have been made to reduce their toxicity, while maintaining high-resolution imaging. Carbon or graphene QDs have been well-researched upon. Fan et al. developed $\mathrm{pH}$-responsive graphene QDs that can distinguish between normal and tumor tissues by difference in fluorescence. These NPs with an average size of around $4 \mathrm{~nm}$ display sharp fluorescence at $\mathrm{pH} 6.8$, which corresponds to the acidic tumor milieu, and can assist in tumor-related surgery [76]. In another study by Feng et al., carbon QDs exposing $\mathrm{NH}_{2}$ group were conjugated to cisplatin prodrug and the resulting module was coupled with an anionic polymer dimethyl maleic acid. In tumor microenvironment, the polymer undergoes charge conversion to cationic, which acts as a trigger for drug release because of charge repulsion. The resultant positively charged carbon dot-prodrug structure has high affinity toward negatively charged cell membrane, resulting in fast internalization [77]. Iron oxide-silica core-shell structure has been used as a scaffold for graphene QDs functionalized with folic acid to increase targetability and enhance imaging [78]. Apart from graphene and carbon QDs, Mn-ZnD QDs have also been reported. Such NPs stabilized with chitosan and modified with folic acid have been studied for selective targetability [79]. Further delineating the versatility of QDs for modification, CdSe/MnS QDs conjugated with peptides containing the asparagine-glycine-arginine (NGR) motif that targets CD13 receptor overexpressed in gliomas were developed [80]. Exploring the potential of lipid NPs in QDs-based theranostics, liposomal delivery system with QDs, targeting inflamed tissues [81] and nanostructured lipid carriers coencapsulating paclitaxel and QDs [82] have been studied recently.

\section{Gold nanoparticles}

AuNPs display a remarkable optical and physical characteristic for more efficient therapy of cancer via compiling both diagnostic and therapeutic functions onto one single platform [83,84]. AuNPs are an interesting agent for x-ray based CT imaging. Small size and easy surface modification to induce functionalization with various targeting moieties have made AuNPs as versatile theranostic system. Moreover, their efficiencies on exhibiting PA signals and photothermal activity have been utilized for imaging and treating tumor progression at early developmental stages, respectively. The targetability of AuNPs can be exploited by conjugating with specific ligands on cancer tissues. AuNPs have the common tendency to aggregate, thus enabling simpler detection in cancerous tissue when excited by light source. The surface plasma resonance of AuNPs is amenable to tuning by altering size, shape and composition of NPs [85,84]. The surface plasma resonance opens up a possibility for characterization of AuNPs by 
surface-enhanced Raman scattering. Raman spectroscopy can give characteristic vibration of the analyzed sample with a narrow spectral distribution [86]. To give an example, AuNPs functionalized with antibody-targeting EGFR have been studied for Raman molecular imaging in in vitro and xenograft models [87]. Also, the optical characteristics of different AuNPs and the fluorescence quenching ability have been investigated extensively in cancer diagnosis because of their sensitivity and selectivity to the tumors through FRET. The FRET-based AuNPs examined the interactions between various AuNPs and biomolecules via finding the fluorescence quenching in donor or signal appearance in acceptor of AuNPs [88]. AuNPs not only can be exploited for imaging the cells but also can be utilized for CT imaging in vivo [89]. Instead of the chemical method-based synthesis of AuNPs, biocompatible, nontoxic plant-leaf-extract-based AuNPs have been used for the delivery of Dox to melanoma and therapeutic efficacy of Dox-encapsulated plant-derived AuNPs is prominent than the free Dox [90]. Higher concentration and smaller size of AuNPs represent higher x-ray attenuation. EPR effect facilitates higher accumulation of specific size AuNPs passively, which in turn can produce stronger x-ray attenuation: a characteristic not usually seen in normal cells but observed in cancerous cells. This differentiation can be a method to achieve diagnosis of tumors in vivo with good spatial image resolution. This approach has been studied in HCC and breast cancer diagnosis [91,92]. To enhance the therapeutic uptake, AuNPs have been conjugated to targeting ligands to facilitate active targeting in a variety of tumors [93-96]. The targeted AuNPs were selectively accumulated on tumor cells and reach tumor sites with higher signal through identification of biomarkers present on cancer cells, and subsequently remaining at the tumor site for prolonged durations. MRI is another impactful imaging technique offering much better resolution than CT [97]. The multifunctionalized AuNPs have been synthesized as dual-modality contrast agents for CT/MR in vitro imaging of breast cancer cells MCF-7, and in vivo imaging human epithelial carcinoma cells' KB-xenografted tumor model, respectively $[98,99]$. Another modality is PET and SPECT imaging. They are excellent imaging models for radiolabeled AuNPs because of using high-sensitivity tissue penetration [100,101]. As an example, Copper-64-alloyed AuNPs have been investigated for cancerous PET imaging due to their simplistic synthesis, stability to radiolabeling, accuracy in diagnosis and immediate systematic clearance [102,103]. These types of AuNPs uphold great possibilities to act as new platforms for multimodality imaging. From a therapeutic point of view, AuNPs have demonstrated interesting results in treating different cancer through PDT/PTT [104]. Systemically administrations of AuNPs have demonstrated low cytotoxicity and high biocompatibility during tumor treatment. As a result, AuNPs-mediated PTT shows promise in treatment of cancer while not compromising on the safety aspect. To give an example, AuNPs loaded with chlorin e 6 are amenable to illumination at $671 \mathrm{~nm}$, followed by excitation, which makes them excellent candidates for simultaneous PDT/PTT of breast cancer in vitro and in vivo. As a result of heating, chlorin e6 molecules are released from AuNPs leading to larger accumulation in cancer site [105]. Ultrafine AuNPs of size $2 \mathrm{~nm}$ have been studied as carriers for the delivery of oligonucleotides in MCF-7 breast cancer cells [106]. Despite the literature on using AuNPs for delivering nucleotides to tumor sites [107], there is still limited data for AuNP-gene theranostics in vivo. Also, the propensity to modulate surface functional groups and high surface area of AuNPs permit conjugation of drugs on surface and controlled release. AuNPs coated with polyethylene glycol (PEG) can be visualized to confer longer circulation times and lower tumor growth [108]. AuNPs surface functionalized by cationic polymers that encapsulate anionic drugs through electrostatic interactions or chemical conjugations with chemotherapeutic drugs also has been reported as carriers that possibly be utilized for cancer therapy [109].

The propensity of optical coherence tomography (OCT) for imaging has been praised for its capacity for high resolution and deep tumor penetration. AuNPs can be conjugated to imaging agents before performing OCT for imaging tumor penetration of the NPs [110,111]. Gold nanoshells as contrast agents have been demonstrated in OCT imaging ex vivo in human breast tissues, offering tumor penetration of upto 300-600 $\mu \mathrm{m}$ [112]. Gold nanorods have also been utilized in OCT to image tumor microvasculature, reaching peak accumulation within $16 \mathrm{~h}$ of injection [113]. In order to enhance the resolution of imaging, contrast fluorescence imaging has been used as a guiding tool to localize OCT imaging in areas, which have been affected primarily by colorectal cancer [114]. Such an approach builds a multimodal platform utilizing the benefits of two imaging facilities onto a single platform. Figure 4 shows a depiction of localization of NPs based on in vivo imaging with hybrid AuNPs tagged with NIR dye [115]. Figure 5 shows the confocal microscopy images taken $24 \mathrm{~h}$ after incubation with drug-Raman NPs [116].

Owing to multitude of characteristics attributed to AuNPs, such as high surface-to-volume ratios, low toxicity and simple surface engineering, they have been extensively studied as theranostics, and hold glaring promise in the biomedical scenario. The electronic and optical properties of AuNPs are important tools to detect tumor tissues and consequently make use of phototherapy. AuNPs functionalized with targeting ligands have been studied for 
(A)

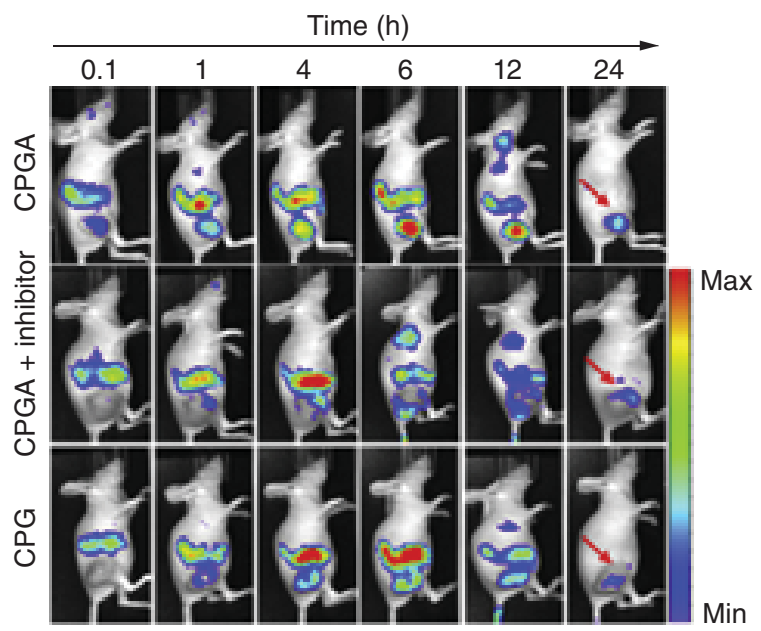

(C)

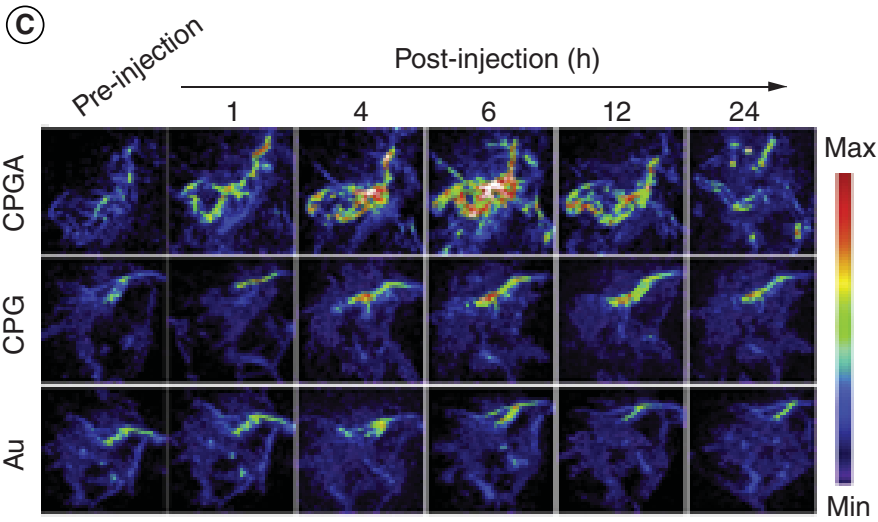

(B)

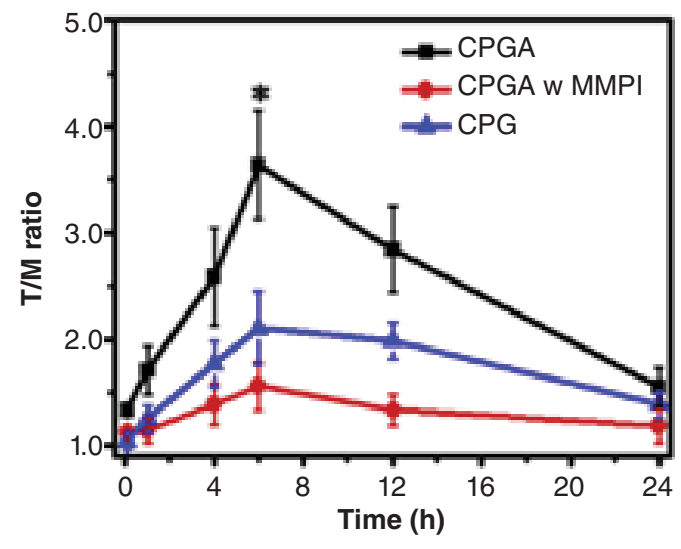

(D)

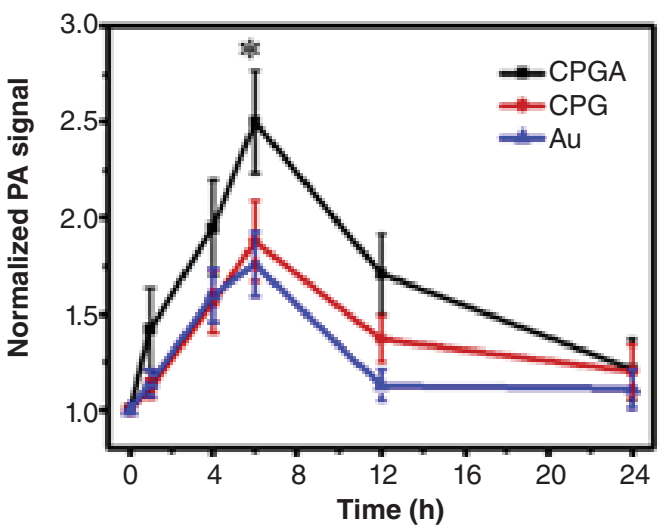

Figure 4. In vivo near-infrared fluorescence and PAT imaging. (A) In vivo NIR fluorescent imaging of tumor-bearing mice taken at various times after intravenous injection of CPGA probe, which was based on graphene oxide-AuNP composite covalently functionalized with NIR dye-labeled MMP substrate. (B) T/M ratio of tumor-bearing mouse model. (C) In vivo photoacoustic imaging of blood vessels in the tumor sites at different time points after intravenous injection. (D) Photoacoustic intensity of tumor tissues at different time points.

AuNP: Gold nanoparticle; CPGA: Tumor-targeted theranostic probe constructed from NIR dye labeled MMP-14 substrate conjugated onto graphene oxide/gold complex; MMP: Matrix metalloproteinase; NIR: Near-infrared; PAT: Photoacoustic tomography; T/M: Tumor/muscle. Reproduced with permission from [115] ๔ 2016.

delivery of genes and anticancer agents. However, more research needs to be done in combating with long-term cytotoxicity and immune system attack in using AuNPs. Thus, it is imperative to exploit new forms of AuNPs to improve their stand in the field of cancer theranostics.

\section{Silica nanoparticles}

Mesoporous silica nanoparticles (MSNPs) provide unique properties to enable multimodality approach of theranostics such as imaging, diagnosis and therapy. The characteristics, such as high surface area and volume, tunable pore size, easily functionalize surface, and biocompatibility, have made them as one of the most promising studied theranostic platform in drug delivery. All the distinct properties of MSNPs support its potential for clinical translation $[117,118]$. Recent reports have been investigated MSNPs and hybridization with other NP platforms, combinations with new diagnostic NPs and dye agents, and their chemical alterations can further support the multifunctional of MSNPs delivery system toward translation. The reason for the use of MSNPs as the platform for theranostic purposes includes three parts: the mesoporous nature or pore size, multifunctional modifiable surface, 

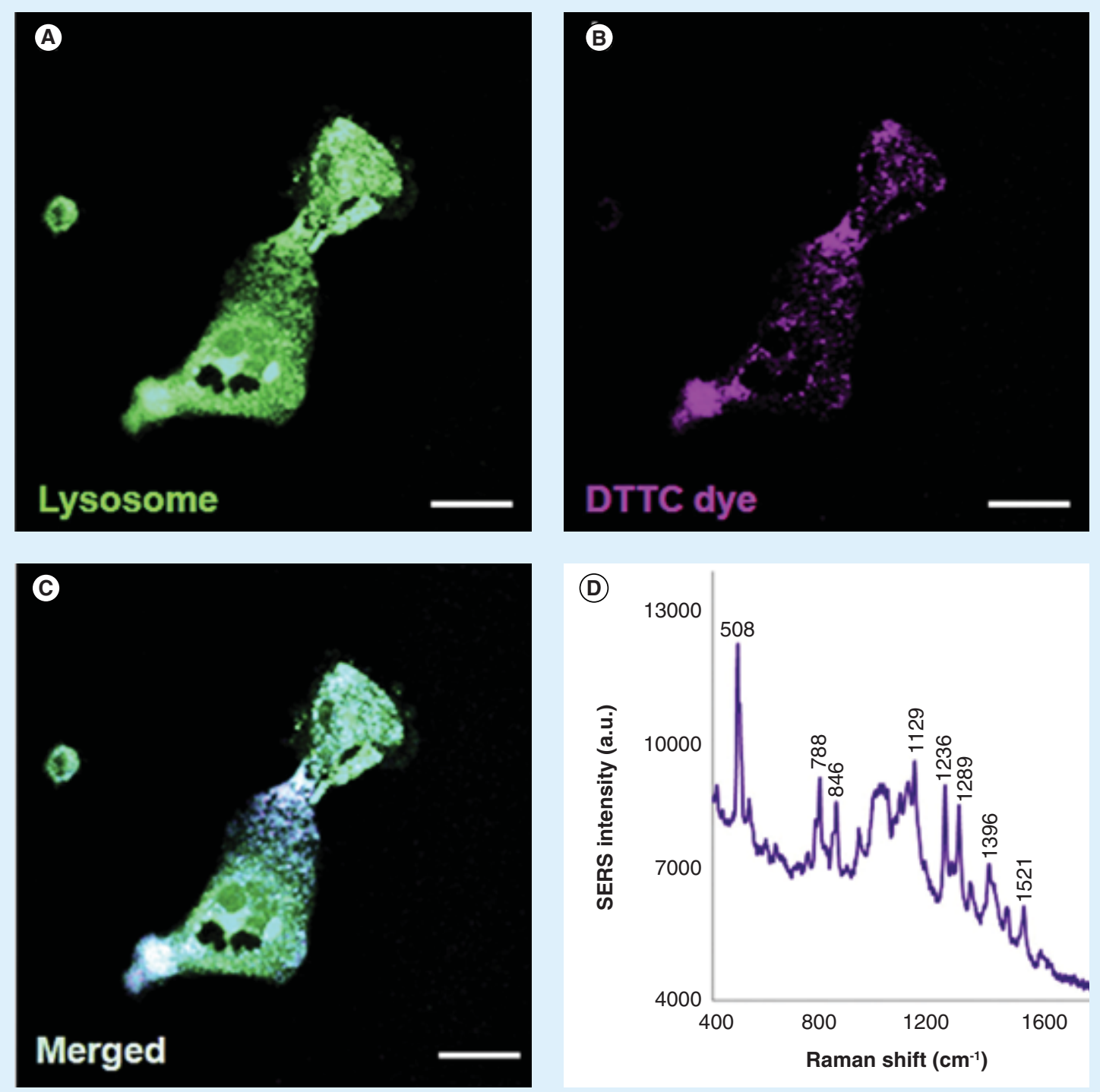

Figure 5. Confocal images of tumor cells incubated for $\mathbf{2 4} \mathrm{h}$ with drug-Raman nanoparticles.

Reproduced with permission from [116] (c) 2014.

and biosafety and biocompatibility. MSNPs have been extensively utilized as delivery nanocarriers of drugs and genes [119-123]. Distinct properties, such as high porosity and surface area of MSNPs, permit high loading of those molecules. The characteristic of the mesoporous area is to identify the drug-loading capacity through the mesopore surface reactions with drug molecules. Moreover, the release patterns of the drug are controllable by mesopore properties such as pore channel geometry. Also, the porous space protects them inside the channeled pores till they reach target and release. Moreover, the surface capping allows controlled release of the therapy, and functionalized surface chemistry permits subsequent conjugation with targeting moieties for active targeting. The MSNPs with optimal drug and gene delivery potential have shown promising result. For example, DOX drug and Bcl-2-targeted siRNA were loaded inside mesopores and on the surface of MSNPs, respectively, to overcome the multiple drug resistance of A2780/AD human ovarian cancer cells [124]. The siRNA delivery effectively had a knockdown of the $\mathrm{Bcl}-2$ gene, leading to suppression of DOX resistance and as a result enhancement of DOX cytotoxicity. The codelivery strategy was effectively showing promising results. Along with that, a codelivery of DOX and Pgp siRNA through PEI-functionalized MSNPs was efficient for the drug-resistant cancer cell line (KB-V1 cells), as the siRNA showed downregulation of the gene expression of the drug exporter, leading to enhance drug cytotoxicity [125]. 


\section{Development in other novel theranostic NPs}

Carbon dots have been widely studied as therapeutic nanoprobes for detection of specific tumor types. As an example, carbon dots tagged with HA and carrying anticancer drug, Dox, have been developed for specific targeting to HA receptors [126]. With the development of safe ultrathin palladium nanosheets, we have opened new avenues for cancer theranostics [127]. Metal-polymer hybrid NPs have been shown to have great promise because of their enhanced light scattering and chemistry [128]. Cornell dots have found their way into clinical trials owing to their superior radioimaging compared with other imaging modalities [129].

\section{Conclusion \& future perspective}

The application of NPs for drug delivery has shown promising growth in the past few years. However, current research on multifunctional NP is focused on improving the drug delivery efficacy and the safety. As new NP systems, such as DTX-encapsulated prostate-specific membrane antigen-targeted NP (BIND-16) for prostate cancer, light-activated NP (AU-011) for ocular melanoma, TNF- $\alpha$ conjugated AuNPs (CYT-6091) for non-smallcell lung cancer and camptothecin-encapsulated polymeric NP (CRLX101) for non-small-cell lung cancers, are brought to clinical trial for the purpose of better therapeutic outcome. It has been reported that there is no sufficient expenditure on nanotechnology research [130]. Considering potential applications of NPs in the health sector, it is urgently required for improving funding. Thus, multifunctional hybrid nanoparticulate drug-delivery systems have the potential to detect cancer progression, deliver drug and reduce side effects for long run.

\section{Acknowledgements}

K Bhise would like to acknowledge a Graduate Research Assistantship for conducting research in U-BiND Systems Laboratory from the Department of Pharmaceutical Sciences, Wayne State University. AK lyer would like to acknowledge Wayne State University Start-up Funding to support the work in U-BiND Systems Laboratory in the Department of Pharmaceutical Sciences.

\section{Financial \& competing interests disclosure}

The authors have no relevant affiliations or financial involvement with any organization or entity with a financial interest in or financial conflict with the subject matter or materials discussed in the manuscript. This includes employment, consultancies, honoraria, stock ownership or options, expert testimony, grants or patents received or pending, or royalties.

No writing assistance was utilized in the production of this manuscript.

\section{Executive summary}

- Nanoparticles (NPs) aiming only for therapy in anticancer domain have been widely studied. However, considering the side effects arising out of unwanted drug localization in nontarget tissues, it is imperative to study the site of drug localization to achieve maximum therapeutic efficacy.

- Theranostics have been recently studied upon with the aim to achieve therapy and diagnosis upon a single platform.

- In this review, recent developments in theranostic NPs like polymer-lipid hybrid, dendrimers, liposomes, polymeric micelles, carbon nanotubes, iron-oxide NPs, quantum dots, gold NPs and silica NPs have been reviewed in detail.

- The review discusses about the current trends in nanotheranostics with recent examples.

\section{References}

1 Facts C. Cancer facts and

figures. (2017) www.cancer.org/research/cancer-facts-statistics/all-cancer-facts-figures/cancer-facts-figures-2017.html

2 Sau S, Banerjee R. Cationic lipid-conjugated dexamethasone as a selective antitumor agent. Eur. J. Med. Chem. 83, 433-447 (2014).

3 Sau S, Mondal SK, Kashaw SK, Iyer AK, Banerjee R. Combination of cationic dexamethasone derivative and STAT3 inhibitor (WP1066) for aggressive melanoma: a strategy for repurposing a Phase I clinical trial drug. Mol. Cell. Biochem. doi:10.1007/s11010-017-3084-z. 1-18 (2017) (Epub ahead of print).

4 Bhise K, Kashaw SK, Sau S, Iyer AK. Nanostructured lipid carriers employing polyphenols as promising anticancer agents: quality by design (QbD) approach. Int. J. Pharm. 526(1-2), 506-515 (2017).

5 Nie S, Xing Y, Kim GJ, Simons JW. Nanotechnology applications in cancer. Annu. Rev. Biomed. Eng. 9(1), 257-288 (2007).

6 Maeda H. The enhanced permeability and retention (EPR) effect in tumor vasculature: the key role of tumor-selective macromolecular drug targeting. Adv. Enzym. Regul. 41(0), 189-207 (2001). 
7 Iyer AK, Singh A, Ganta S, Amiji MM. Role of integrated cancer nanomedicine in overcoming drug resistance. Adv. Drug Deliv. Rev. 65(13), 1784-1802 (2013).

8 Iyer AK, Greish K, Seki T et al. Polymeric micelles of zinc protoporphyrin for tumor targeted delivery based on EPR effect and singlet oxygen generation. J. Drug Target. 15(September), 496-506 (2007).

9 Amjad MW, Kesharwani P, Mohd Amin MCI, Iyer AK. Recent advances in the design, development, and targeting mechanisms of polymeric micelles for delivery of siRNA in cancer therapy. Prog. Polym. Sci. 64, 154-181 (2017).

10 Iyer AK, Khaled G, Fang J, Maeda H. Exploiting the enhanced permeability and retention effect for tumor targeting. Drug Discov. Today. 11(17), 812-818 (2006)

11 Greish K, Iyer AK, Fang J, Kawasuji M, Maeda H. Enhanced permeability and retention (EPR) effect and tumor-selective delivery of anticancer drugs. In:Delivery of Protein and Peptide Drugs in Cancer (Volume 10:14). Vladimir P Torchilin (Ed.). World Scientific Publishing Co., 37-52 (2005).

12 Sau S, Alsaab HO, Kashaw SK, Tatiparti K, Iyer AK. Advances in antibody-drug conjugates: a new era of targeted cancer therapy. Drug Discov. Today (2017). http://dx.doi.org/10.1016/j.drudis.2017.05.011 (Epub ahead of print).

13 Alsaab HO, Sau S, Alzhrani R et al. PD-1 and PD-L1 checkpoint signaling inhibition for cancer immunotherapy: mechanism, combinations, and clinical outcome. Front. Pharmacol. 8(August), 1-15 (2017). doi:10.3389/fphar.2017.00561

14 Lammers T, Subr V, Ulbrich K, Hennink WE, Storm G, Kiessling F. Polymeric nanomedicines for image-guided drug delivery and tumor-targeted combination therapy. Nano Today 5(3), 197-212 (2010).

15 Iyer AK, Su Y, Feng J et al. The effect of internalizing human single chain antibody fragment on liposome targeting to epithelioid and sarcomatoid mesothelioma. Biomaterials 32(10), 2605-2613 (2011).

16 Iyer AK, Lan X, Zhu X et al. Novel human single chain antibody fragments that are rapidly internalizing effectively target epithelioid and sarcomatoid mesotheliomas. Cancer Res. 71(7), 2428-2432 (2011).

17 He J, Wang Y, Feng J et al. Targeting prostate cancer cells in vivo using a rapidly internalizing novel human single-chain antibody fragment. J. Nucl. Med. 51(3), 427 (2010).

18 Abeylath SC, Ganta S, Iyer AK, Amiji M. Combinatorial-designed multifunctional polymeric nanosystems for tumor-targeted therapeutic delivery. Acc. Chem. Res. 44(10), 1009-1017 (2011).

19 Sailor Michael J, Park JH. Hybrid nanoparticles for detection and treatment of cancer. Adv. Mater. 24(28), 3779-3802 (2013).

20 Dehaini D, Fang RH, Luk BT et al. Ultra-small lipid-polymer hybrid nanoparticles for tumor-penetrating drug delivery. Nanoscale. 8(30), 14411-14419 (2016).

21 Reisch A, Runser A, Arntz Y, Mély Y, Klymchenko AS. Charge-controlled nanoprecipitation as a modular approach to ultrasmall polymer nanocarriers: making bright and stable nanoparticles. ACS Nano. 9(5), 5104-5116 (2015).

22 Luong D, Kesharwani P, Deshmukh R et al. PEGylated PAMAM dendrimers: enhancing efficacy and mitigating toxicity for effective anticancer drug and gene delivery. Acta Biomater. 43, 14-29 (2016).

23 Lee CC, MacKay JA, Fréchet JMJ, Szoka FC. Designing dendrimers for biological applications. Nat. Biotechnol. 23(12), 1517-1526 (2005).

24 Thomas TP, Shukla R, Kotlyar A, Kukowska-Latallo J, Baker JR. Dendrimer-based tumor cell targeting of fibroblast growth factor-1. Bioorg. Med. Chem. Lett. 20(2), 700-703 (2010).

25 Ki Choi S, Thomas T, Li MH, Kotlyar A, Desai A, Baker J. Light-controlled release of caged doxorubicin from folate receptor-targeting PAMAM dendrimer nanoconjugate. Chem. Commun. 46(15), 2632-2634 (2010).

26 Guillaudeu SJ, Fox ME, Haidar YM, Dy EE, Szoka FC, Fréchet JMJ. PEGylated dendrimers with core functionality for biological applications. Bioconjug. Chem. 19(2), 461-469 (2008).

27 Almutairi A, Rossin R, Shokeen M et al. Biodegradable dendritic positron-emitting nanoprobes for the noninvasive imaging of angiogenesis. Proc. Natl Acad. Sci. USA 106(3), 685-90 (2009).

28 Zhu J, Zheng L, Wen S et al. Targeted cancer theranostics using alpha-tocopheryl succinate-conjugated multifunctional dendrimer-entrapped gold nanoparticles. Biomaterials 35(26), 7635-7646 (2014).

29 Taratula O, Schumann C, Duong T, Taylor KL, Taratula O. Dendrimer-encapsulated naphthalocyanine as a single agent-based theranostic nanoplatform for near-infrared fluorescence imaging and combinatorial anticancer phototherapy. Nanoscale 7(9), 3888-3902 (2015).

30 Zhu J, Fu F, Xiong Z, Shen M, Shi X. Dendrimer-entrapped gold nanoparticles modified with RGD peptide and alpha-tocopheryl succinate enable targeted theranostics of cancer cells. Colloids Surfaces B Biointerfaces 133, 36-42 (2015).

31 Petersen AL, Henriksen JR, Binderup T et al. In vivo evaluation of PEGylated 64Cu-liposomes with theranostic and radiotherapeutic potential using micro PET/CT. Eur. J. Nucl. Med. Mol. Imaging 43(5), 941-952 (2016).

32 Pang X, Wang J, Tan X et al. Dual-modal imaging-guided theranostic nanocarriers based on indocyanine green and mTOR inhibitor rapamycin. ACS Appl. Mater. Interfaces 8(22), 13819-13829 (2016). 
33 Huang Y, Hemmer E, Rosei F, Vetrone F. Multifunctional liposome nanocarriers combining upconverting nanoparticles and anticancer drugs. J. Phys. Chem. B. 120(22), 4992-5001 (2016).

34 Duan Y, Wei L, Petryk J, Ruddy TD. Formulation, characterization and tissue distribution of a novel pH-sensitive long-circulating liposome-based theranostic suitable for molecular imaging and drug delivery. Int. J. Nanomedicine 11, 5697-5708 (2016).

35 Sonali, Singh RP, Sharma G et al. RGD-TPGS decorated theranostic liposomes for brain targeted delivery. Colloids Surfaces $B$ Biointerfaces 147, 129-141 (2016).

36 Sonali S, Singh RP, Singh N et al. Transferrin liposomes of docetaxel for brain targeted cancer applications: formulation and brain theranostics. Drug Deliv. 7544(March), 1-37 (2016).

37 Feng L, Tao D, Dong Z et al. Near-infrared light activation of quenched liposomal Ce6 for synergistic cancer phototherapy with effective skin protection. Biomaterials. 127, 13-24 (2017).

38 Sivasubramanian K, Mathiyazhakan M, Wiraja C, Upputuri PK, Xu C, Pramanik M. Near-infrared light-responsive liposomal contrast agent for photoacoustic imaging and drug release applications. J. Biomed. Opt. 22(4), 41007 (2016).

39 Alsaab H, Alzhrani R, Kesharwani P, Sau S, Boddu S, Iyer A. Folate decorated nanomicelles loaded with a potent curcumin analogue for targeting retinoblastoma. Pharmaceutics 9(2), 15 (2017). www.mdpi.com/1999--4923/9/2/15

40 Northeastern University.WO2010042823 A1 (2010).

41 Ganesh S, Iyer AK, Morrissey DV, Amiji MM. Hyaluronic acid based self-assembling nanosystems for CD44 target mediated siRNA delivery to solid tumors. Biomaterials 34(13), 3489-3502 (2013).

42 Sahu P, Kashaw SK, Jain S, Sau S, Iyer AK. Assessment of penetration potential of pH responsive double walled biodegradable nanogels coated with eucalyptus oil for the controlled delivery of 5-fluorouracil: in vitro and ex vivo studies. J. Control. Rel. 253, 122-136 (2017). www.ncbi.nlm.nih.gov/pubmed/28322977

43 Li Y, Ma J, Zhu H, Gao X, Dong H, Shi D. Green synthetic, multifunctional hybrid micelles with shell embedded magnetic nanoparticles for theranostic applications. ACS Appl. Mater. Interfaces 5(15), 7227-7235 (2013).

44 Sahu P, Kashaw SK, Jain S, Sau S, Iyer AK. pH responsive biodegradable nanogels for sustained release of bleomycin. Bioorganic \& Medicinal Chemistry 25, 4595-4613 (2017). https://doi.org/10.1016/j.bmc.2017.06.038

45 Xiao Y, Hong H, Javadi A et al. Multifunctional unimolecular micelles for cancer-targeted drug delivery and positron emission tomography imaging. Biomaterials 33(11), 3071-3082 (2012).

46 Iyer AK, He J. Radiolabeled oligonucleotides for antisense imaging. Curr. Org. Synth. 8(4), 604-614 (2011).

47 Yang $\mathrm{H}$, Mao H, Wan $\mathrm{Z}$ et al. Micelles assembled with carbocyanine dyes for theranostic near-infrared fluorescent cancer imaging and photothermal therapy. Biomaterials 34(36), 9124-9133 (2013).

48 Muthu MS, Kutty RV, Luo Z, Xie J, Feng SS. Theranostic vitamin E TPGS micelles of transferrin conjugation for targeted co-delivery of docetaxel and ultra bright gold nanoclusters. Biomaterials 39, 234-248 (2015).

49 Kumar R, Kulkarni A, Nagesha DK, Sridhar S. In vitro evaluation of theranostic polymeric micelles for imaging and drug delivery in cancer. Theranostics 2(7), 714-722 (2012).

50 Guo M, Mao H, Li Y et al. Dual imaging-guided photothermal/ photodynamic therapy using micelles. Biomaterials 35(16), 4656-4666 (2014).

$51 \mathrm{Li}$ W, Zheng C, Pan Z et al. Smart hyaluronidase-actived theranostic micelles for dual-modal imaging guided photodynamic therapy. Biomaterials 101, 10-19 (2016).

52 Wickens JM, Alsaab HO, Kesharwani P et al. Recent advances in hyaluronic acid-decorated nanocarriers for targeted cancer therapy. Drug Discov. Today 22(4), 665-680 (2016).

53 Howell M, Mallela J, Wang C et al. Manganese-loaded lipid-micellar theranostics for simultaneous drug and gene delivery to lungs. J. Control. Rel. 167(2), 210-218 (2013).

54 Wang $\mathrm{T}$, Wang D, Yu H et al. Intracellularly acid-switchable multifunctional micelles for combinational photo/chemotherapy of the drug-resistant tumor. ACS Nano. 10(3), 3496-3508 (2016).

55 Tan A, Yildirimer L, Rajadas J, De La Pena H, Pastorin G, Seifalian A. Quantum dots and carbon nanotubes in oncology: a review on emerging theranostic applications in nanomedicine. Nanomedicine 6(6), 1101-1114 (2011).

56 Liu J, Wang C, Wang X et al. Mesoporous silica coated single-walled carbon nanotubes as a multifunctional light-responsive platform for cancer combination therapy. Adv. Funct. Mater. 25(3), 384-392 (2015).

57 Murakami T, Nakatsuji H, Inada $\mathrm{M}$ et al. Photodynamic and photothermal effects of semiconducting and metallic-enriched single-walled carbon nanotubes. J. Am. Chem. Soc. 134(43), 17862-17865 (2012).

58 Hashimoto Y, Abu Lila AS, Shimizu T, Ishida T, Kiwada H. B cell-intrinsic toll-like receptor 7 is responsible for the enhanced anti-PEG IgM production following injection of siRNA-containing PEGylated lipoplex in mice. J. Control. Rel. 184(1), 1-8 (2014).

59 Abu Lila AS, Kiwada H, Ishida T. The accelerated blood clearance (ABC) phenomenon: clinical challenge and approaches to manage. J. Control. Rel. 172(1), 38-47 (2013). 
60 Xie L, Wang G, Zhou $\mathrm{H}$ et al. Functional long circulating single walled carbon nanotubes for fluorescent/photoacoustic imaging-guided enhanced phototherapy. Biomaterials 103, 219-228 (2016).

61 Liang X, Shang W, Chi C et al. Dye-conjugated single-walled carbon nanotubes induce photothermal therapy under the guidance of near-infrared imaging. Cancer Lett. 383(2), 243-249 (2016).

62 Zhao H, Chao Y, Liu J et al. Polydopamine coated single-walled carbon nanotubes as a versatile platform with radionuclide labeling for multimodal tumor imaging and therapy. Theranostics 6(11), 1833-1843 (2016).

63 Wen L, Ding W, Yang S, Xing D. Microwave pumped high-efficient thermoacoustic tumor therapy with single wall carbon nanotubes. Biomaterials 75, 163-173 (2016).

64 Wang L, Zhang M, Zhang N et al. Synergistic enhancement of cancer therapy using a combination of docetaxel and photothermal ablation induced by single-walled carbon nanotubes. Int. J. Nanomedicine 6, 2641-2652 (2011).

$65 \mathrm{Li} \mathrm{R}$, Wu R, Zhao L et al. Folate and iron difunctionalized multiwall carbon nanotubes as dual-targeted drug nanocarrier to cancer cells. Carbon NY 49(5), 1797-1805 (2011).

66 Li J, Wang S, Shi X, Shen M. Aqueous-phase synthesis of iron oxide nanoparticles and composites for cancer diagnosis and therapy. Adv. Colloid Interface Sci. (2017).doi:10.1016/j.cis.2017.02.009

67 Unterweger $\mathrm{H}$, Tietze R, Janko C et al. Development and characterization of magnetic iron oxide nanoparticles with a cisplatin-bearing polymer coating for targeted drug delivery. Int. J. Nanomedicine 9, 3659-3676 (2014).

68 Yang R-M, Fu C, Fang J et al. Hyaluronan-modified superparamagnetic iron oxide nanoparticles for bimodal breast cancer imaging and photothermal therapy. Int. J. Nanomedicine 12, 197-206 (2016).

69 Azhdarzadeh M, Atyabi F, Saei AA et al. Theranostic MUC-1 aptamer targeted gold coated superparamagnetic iron oxide nanoparticles for magnetic resonance imaging and photothermal therapy of colon cancer. Colloids Surfaces B Biointerfaces 143, 224-232 (2016).

70 Wang Z, Qiao R, Tang N et al. Active targeting theranostic iron oxide nanoparticles for MRI and magnetic resonance-guided focused ultrasound ablation of lung cancer. Biomaterials 127, 25-35 (2017).

71 Tatiparti K, Sau S, Kashaw S, Iyer A. siRNA delivery strategies: a comprehensive review of recent developments. Nanomaterials $7(4), 77$ (2017). www.mdpi.com/2079--4991/7/4/77

72 Jiang S, Eltoukhy A, Love K, Langer R, Anderson D. Lipidoid-coated iron oxide nanoparticles for efficient DNA and siRNA delivery. Nano Lett.13(3), 1059-1064 (2013).

73 Luong D, Sau S, Kesharwani P, Iyer AK. Polyvalent folate-dendrimer-coated iron oxide theranostic nanoparticles for simultaneous magnetic resonance imaging and precise cancer cell targeting. Biomacromolecules 18(4), 1197-1209 (2017).

74 Huang Y, Mao K, Zhang B, Zhao Y. Superparamagnetic iron oxide nanoparticles conjugated with folic acid for dual target-specific drug delivery and MRI in cancer theranostics. Mater. Sci. Eng. C. 70, 763-771 (2017).

75 Trabulo Sara, Aires Antonio, Aicher Alexandra, Heeschen Christopher, Cortajarena Aitziber L. Multifunctionalized iron oxide nanoparticles for selective targeting of pancreatic cancer cells. Biochim. Biophys. Acta 1861(6),1597-1605 (2017).

76 Fan Z, Zhou S, Garcia C, Fan L, Zhou J. pH-Responsive fluorescent graphene quantum dots for fluorescence-guided cancer surgery and diagnosis. Nanoscale 13;9(15), 4928-4933 (2017).

77 Feng T, Ai X, An G, Yang P, Zhao Y. Charge-convertible carbon dots for imaging-guided drug delivery with enhanced in vivo cancer therapeutic efficiency. ACS Nano. 10(4), 4410-4420 (2016).

78 Su X, Chan C, Shi J et al. A graphene quantum dot@Fe3O4@SiO2 based nanoprobe for drug delivery sensing and dual-modal fluorescence and MRI imaging in cancer cells. Biosens. Bioelectron. 92, 489-495 (2016).

79 Bwatanglang IB, Mohammad F, Yusof NA et al. Folic acid targeted Mn:ZnS quantum dots for theranostic applications of cancer cell imaging and therapy. Int. J. Nanomedicine 11, 413-428 (2016).

80 Huang N, Cheng S, Zhang X et al. Efficacy of NGR peptide-modified PEGylated quantum dots for crossing the blood-brain barrier and targeted fluorescence imaging of glioma and tumor vasculature. Nanomedicine 13(1), 83-93 (2017).

81 Aizik G, Waiskopf N, Agbaria M, Levi-Kalisman Y, Banin U, Golomb G. Delivery of liposomal quantum dots via monocytes for imaging of inflamed tissue. ACS Nano. acsnano.7b00016 (2017) (Epub ahead of print).

82 David L, Liu Y, Zhang B et al. Near-infrared mediated quantum dots and paclitaxel co-loaded nanostructured lipid carriers for cancer theragnostic. Colloids Surfaces B Biointerfaces 150, 121-130 (2016).

83 Sau S, Agarwalla P, Mukherjee $S$ et al. Cancer cell-selective promoter recognition accompanies antitumor effect by glucocorticoid receptor-targeted gold nanoparticle. Nanoscale 6(12), 6745-54 (2014). www.ncbi.nlm.nih.gov/pubmed/24824564.

84 Choi Y, Cho Y, Kim M, Grailhe R, Song R. Fluorogenic quantum dot-gold nanoparticle assembly for beta secretase inhibitor screening in live cell. Anal. Chem. 84(20), 8595-8601 (2012).

85 Mollasalehi H, Yazdanparast R. Non-crosslinking gold nanoprobes for detection of nucleic acid sequence-based amplification products. Anal. Biochem. 425(2), 91-95 (2012).

86 Nguyen DT, Kim D-J, Kim K-S. Controlled synthesis and biomolecular probe application of gold nanoparticles. Micron 42(3), 207-227 (2011). 
87 Jokerst JV, Miao Z, Zavaleta C, Cheng Z, Gambhir SS. Affibody-functionalized gold-silica nanoparticles for Raman molecular imaging of the epidermal growth factor receptor. Small 7(5), 625-633 (2011).

88 Saha K, Agasti SS, Kim C, Li X, Rotello VM. Gold nanoparticles in chemical and biological sensing. Chem. Rev. 112(5), 2739-2779 (2012).

89 Petros RA, DeSimone JM. Strategies in the design of nanoparticles for therapeutic applications. Nat. Rev. Drug Discov. 9(8), 615-627 (2010).

90 Mukherjee S, Sau S, Madhuri D et al. Green synthesis and characterization of monodispersed gold nanoparticles: toxicity study, delivery of doxorubicin and its bio-distribution in mouse model. J. Biomed. Nanotechnol. 12(1), 165-181 (2016).

91 Lusic H, Grinstaff MW. X-ray-computed tomography contrast agents. Chem. Rev. 113(3), 1641-1666 (2012).

92 Ghaghada KB, Badea CT, Karumbaiah L et al. Evaluation of tumor microenvironment in an animal model using a nanoparticle contrast agent in computed tomography imaging. Acad. Radiol. 18(1), 20-30 (2011).

93 Wen S, Li K, Cai $\mathrm{H}$ et al. Multifunctional dendrimer-entrapped gold nanoparticles for dual mode CT/MR imaging applications. Biomaterials 34(5), 1570-1580 (2013).

94 Wang H, Zheng L, Peng C, Shen M, Shi X, Zhang G. Folic acid-modified dendrimer-entrapped gold nanoparticles as nanoprobes for targeted CT imaging of human lung adencarcinoma. Biomaterials 34(2), 470-480 (2013).

95 Reuveni T, Motiei M, Romman Z, Popovtzer A, Popovtzer R. Targeted gold nanoparticles enable molecular CT imaging of cancer: an in vivo study. Int. J. Nanomedicine 6(2859), e64 (2011).

96 Hainfeld JF, O'connor MJ, Dilmanian FA, Slatkin DN, Adams DJ, Smilowitz HM. Micro-CT enables microlocalisation and quantification of Her2-targeted gold nanoparticles within tumour regions. Br. J. Radiol. 84(1002), 526-533 (2014).

$97 \mathrm{Li} \mathrm{J}, \mathrm{Hu} \mathrm{Y}$, Yang J et al. Hyaluronic acid-modified $\mathrm{Fe}_{3} \mathrm{O}_{4} @$ Au core/shell nanostars for multimodal imaging and photothermal therapy of tumors. Biomaterials 38, 10-21 (2015).

98 Li K, Wen S, Larson AC et al. Multifunctional dendrimer-based nanoparticles for in vivo MR/CT dual-modal molecular imaging of breast cancer. Int. J. Nanomedicine 8, 2589-2600 (2013).

99 Chen Q, Li K, Wen S et al. Targeted CT/MR dual mode imaging of tumors using multifunctional dendrimer-entrapped gold nanoparticles. Biomaterials 34(21), 5200-5209 (2013).

100 Karmani L, Labar D, Valembois V et al. Antibody-functionalized nanoparticles for imaging cancer: influence of conjugation to gold nanoparticles on the biodistribution of 89Zr-labeled cetuximab in mice. Contrast Media Mol. Imaging 8(5), 402-408 (2013).

101 Karmani L, Bouchat V, Bouzin C et al. 89Zr-labeled anti-endoglin antibody-targeted gold nanoparticles for imaging cancer: implications for future cancer therapy. Nanomedicine 9(13), 1923-1937 (2014).

102 Zhao Y, Sultan D, Detering L, Luehmann H, Liu Y. Facile synthesis, pharmacokinetic and systemic clearance evaluation, and positron emission tomography cancer imaging of $64 \mathrm{Cu}-$ Au alloy nanoclusters. Nanoscale 6(22), 13501-13509 (2014).

103 Zhao Y, Sultan D, Detering L et al. Copper-64-alloyed gold nanoparticles for cancer imaging: improved radiolabel stability and diagnostic accuracy. Angew. Chemie Int. Ed. 53(1), 156-159 (2014).

104 Choi KY, Liu G, Lee S, Chen X. Theranostic nanoplatforms for simultaneous cancer imaging and therapy: current approaches and future perspectives. Nanoscale 4(2), 330-342 (2012).

105 Lin J, Wang S, Huang P et al. Photosensitizer-loaded gold vesicles with strong plasmonic coupling effect for imaging-guided photothermal/photodynamic therapy. ACS Nano. 7(6), 5320-5329 (2013).

106 Huo S, Jin S, Ma X et al. Ultrasmall gold nanoparticles as carriers for nucleus-based gene therapy due to size-dependent nuclear entry. ACS Nano. 8(6), 5852-5862 (2014).

107 Bishop CJ, Tzeng SY, Green JJ. Degradable polymer-coated gold nanoparticles for co-delivery of DNA and siRNA. Acta Biomater. 11, 393-403 (2015).

108 Thakor AS, Jokerst J, Zavaleta C, Massoud TF, Gambhir SS. Gold nanoparticles: a revival in precious metal administration to patients. Nano Lett. 11(10), 4029-4036 (2011).

109 Bigall NC, Curcio A, Leal MP et al. Magnetic nanocarriers with tunable $\mathrm{pH}$ dependence for controlled loading and release of cationic and anionic payloads. Adv. Mater. 23(47), 5645-5650 (2011).

110 Coughlin AJ, Ananta JS, Deng N, Larina IV, Decuzzi P, West JL. Gadolinium-conjugated gold nanoshells for multimodal diagnostic imaging and photothermal cancer therapy. Small 10(3), 556-565 (2014).

111 Terentyuk G, Panfilova E, Khanadeev V et al. Gold nanorods with a hematoporphyrin-loaded silica shell for dual-modality photodynamic and photothermal treatment of tumors in vivo. Nano Res. 7(3), 325-337 (2014).

112 Zhou C, Tsai T-H, Adler DC et al. Photothermal optical coherence tomography in ex vivo human breast tissues using gold nanoshells. Opt. Lett. 35(5), 700-702 (2010).

113 Tucker-Schwartz JM, Beavers KR, Sit WW, Shah AT, Duvall CL, Skala MC. In vivo imaging of nanoparticle delivery and tumor microvasculature with multimodal optical coherence tomography. Biomed. Opt. Express 5(6), 1731-1743 (2014). 
114 Iftimia N, Iyer AK, Hammer DX et al. Fluorescence-guided optical coherence tomography imaging for colon cancer screening: a preliminary mouse study. Biomed. Opt. Express 3(1), 178 (2012).

115 Gao S, Zhang L, Wang G et al. Hybrid graphene/Au activatable theranostic agent for multimodalities imaging guided enhanced photothermal therapy. Biomaterials 79, 36-45 (2016).

116 Conde J, Bao C, Cui D, Baptista PV, Tian F. Antibody-drug gold nanoantennas with Raman spectroscopic fingerprints for in vivo tumour theranostics. J. Control. Rel. 183(1), 87-93 (2014).

117 Wang Y, Zhao Q, Han N et al. Mesoporous silica nanoparticles in drug delivery and biomedical applications. Nanomedicine 11(2), 313-327 (2015).

118 Baek S, Singh RK, Khanal D et al. Smart multifunctional drug delivery towards anticancer therapy harmonized in mesoporous nanoparticles. Nanoscale 7(34), 14191-14216 (2015).

119 Slowing II, Vivero-Escoto JL, Wu C-W, Lin VS-Y. Mesoporous silica nanoparticles as controlled release drug delivery and gene transfection carriers. Adv. Drug Deliv. Rev. 60(11), 1278-1288 (2008).

120 Patel KD, Mahapatra C, Jin G-Z, Singh RK, Kim H-W. Biocompatible mesoporous nanotubular structured surface to control cell behaviors and deliver bioactive molecules. ACS Appl. Mater. Interfaces. 7(48), 26850-26859 (2015).

121 Niu Y, Yu M, Zhang J et al. Synthesis of silica nanoparticles with controllable surface roughness for therapeutic protein delivery. J. Mater. Chem. B. 3(43), 8477-8485 (2015).

122 Kim T-H, Singh RK, Kang MS, Kim J-H, Kim H-W. Inhibition of osteoclastogenesis through siRNA delivery with tunable mesoporous bioactive nanocarriers. Acta Biomater. 29, 352-364 (2016).

123 Argyo C, Weiss V, Bräuchle C, Bein T. Multifunctional mesoporous silica nanoparticles as a universal platform for drug delivery. Chem. Mater. 26(1), 435-451 (2013).

124 Chen AM, Zhang M, Wei D et al. Co-delivery of doxorubicin and Bcl-2 siRNA by mesoporous silica nanoparticles enhances the efficacy of chemotherapy in multidrug-resistant cancer cells. Small 5(23), 2673-2677 (2009).

125 Meng H, Liong M, Xia T et al. Engineered design of mesoporous silica nanoparticles to deliver doxorubicin and Pgp siRNA to overcome drug resistance in a cancer cell line. ACS Nano. 4(8), 4539 (2010).

126 Gao N, Yang W, Nie H et al. Turn-on theranostic fluorescent nanoprobe by electrostatic self-assembly of carbon dots with doxorubicin for targeted cancer cell imaging, in vivo hyaluronidase analysis, and targeted drug delivery. Biosens. Bioelectron. 96(May), 300-307 (2017).

127 Chen M, Chen S, He C et al. Safety profile of two-dimensional Pd nanosheets for photothermal therapy and photoacoustic imaging. Nano Res. 10(4), 1234-1248 (2017).

128 Capek I. Polymer decorated gold nanoparticles in nanomedicine conjugates. Adv. Colloid Interface Sci.(2017).doi.org/10.1016/j.cis.2017.01.007

129 Chakravarty R, Goel S, Dash A, Cai W. Radiolabeled inorganic nanoparticles for positron emission tomography imaging of cancer: an overview. J. Nucl. Med. Mol. Imaging 67(2), 181-204 (2017).

130 Bharali DJ, Khalil M, Gurbuz M, Simone TM, Mousa SA. Nanoparticles and cancer therapy: a concise review with emphasis on dendrimers. Int. J. Nanomedicine 4(1), 1-7 (2009).

131 Zhang L, Chan JM, Gu FX et al. Self-assembled lipid polymer hybrid nanoparticles: a robust drug delivery platform. ACS Nano. 2(8), 1696-1702 (2008).

132 Patri AK, Majoros IJ, Baker JR. Dendritic polymer macromolecular carriers for drug delivery. Curr. Opin. Chem. Biol. 6(4), 466-471 (2002).

133 Saw PE, Park J, Jon S, Farokhzad OC. A drug-delivery strategy for overcoming drug resistance in breast cancer through targeting of oncofetal fibronectin. Nanomedicine 13(2), 713-722 (2017).

134 Li Z, de Barros ALB, Soares DCF, Moss SN, Alisaraie L. Functionalized single-walled carbon nanotubes: cellular uptake, biodistribution and applications in drug delivery. Int. J. Pharm. 524(1-2), 41-54 (2017). 